\title{
Low-Power Smart Imagers for Vision-Enabled Sensor Networks
}





"Any sufficiently advanced technology is indistinguishable from magic" Arthur C. Clarke 



\section{Contents}

List of Figures $\quad$ xi

List of Tables $\quad$ xv

$\begin{array}{ll}\text { Abbreviations } & \text { xvii }\end{array}$

1 Introduction 1

1.1 Ubiquitous computing: a new era in computer history . . . . . . . . . . . . . 1

1.2 Wireless Sensor Networks: a basic building block . . . . . . . . . . . . . . . . . 2

1.3 Vision: a step forward in WSNs . . . . . . . . . . . . . . . . . 2

2 Vision-enabled WSN nodes: state of the art $\quad 5$

2.1 A brief introduction to WSNs . . . . . . . . . . . . . . . . 5

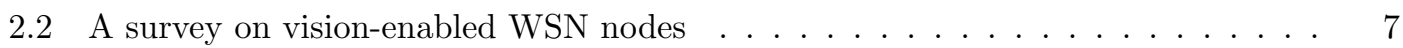

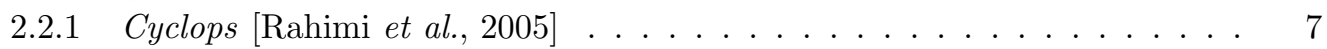

2.2.2 Imote2-based platform [Teixeira et al., 2006] . . . . . . . . . . . . . . 9

2.2.3 MeshEye ${ }^{\mathrm{TM}}[$ Hengstler et al., 2007] . . . . . . . . . . . . . 10

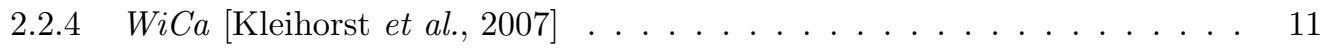

2.2.5 FireFly Mosaic [Rowe et al., 2007b] . . . . . . . . . . . . . . 13

2.2.6 CITRIC [Chen et al., 2008] . . . . . . . . . . . . . . 14

2.2.7 EyeRIS ${ }^{\mathrm{TM}}$-based platform [Bakkali et al., 2010] . . . . . . . . . . 16

2.3 Comparative analysis . . . . . . . . . . . . . . . . . . 17

2.4 Guidelines for a power-aware vision-enabled wireless node . . . . . . . . . . . 18

3 Processing primitives for image simplification $\quad 23$

3.1 Gaussian filtering . . . . . . . . . . . . . . . . . . . . . . . . . . . . . .

3.1.1 Continuous-space linear diffusion . . . . . . . . . . . . . . . 24

3.1.2 Discrete-space linear diffusion . . . . . . . . . . . . . . . . . . 25

3.1.2.1 Scale-space representation . . . . . . . . . . . . . . . . 28

3.1.2.2 Gaussian pyramids . . . . . . . . . . . . . . 28

3.2 Reconfigurable block-wise image plane division . . . . . . . . . . . . . . . . . . . . . . . . . . . . . . . . . .

3.3 Multiresolution image representation . . . . . . . . . . . . . . . . . . . . . . . . . . . . . 30

3.4 Image pre-distortion for reduced kernel filtering . . . . . . . . . . . . . . . . . . . . . . . . . . . . . . . .

3.5 Energy-based scene representation . . . . . . . . . . . . . . . . . . . . . . . . . . . . . . . . .

3.6 Summary . . . . . . . . . . . . . . . . . . . 36

4 VLSI implementation of linear diffusion $\quad 39$

4.1 Introduction . . . . . . . . . . . . . . . . . . . . . . 39

4.2 Linear diffusion in transconductance-based CNNs . . . . . . . . . . . . . . . . . . . . . . . . . . . . . . .

4.2.1 Unconstrained linear diffusion in $\mathrm{CNNs}$. . . . . . . . . . . . . . 41

4.2.1.1 Mapping of the diffusion equation into the CNN grid . . . . . 41 
4.2.1.2 State equations . . . . . . . . . . . . . . . 41

4.2.1.3 Effect of mismatch: analysis of a 2-cell CNN . . . . . . . . . . . 44

4.2.1.4 Numerical extrapolation of the results . . . . . . . . . . . . . 46

4.2 .2 Constrained linear diffusion in CNNs . . . . . . . . . . . . . . . . 48

4.2.2.1 Linear diffusion through a resistive grid . . . . . . . . . . . . 48

4.2.2.2 Templates for constrained diffusion . . . . . . . . . . . . . 50

4.2.2.3 Modified state equations . . . . . . . . . . . . . 50

4.2.2.4 Effects of mismatch in a 2-cell CNN with $\lambda>0$. . . . . . . 51

4.2.2.5 Numerical results for larger networks . . . . . . . . . . . . 52

4.2.3 Comparison between time-controlled unconstrained diffusion and constrained diffusion . . . . . . . . . . . . . . . . 53

4.2.4 Physical origin for the non-convergence of the networks . . . . . . . . 58

4.3 Linear diffusion in time-controlled RC networks . . . . . . . . . . . . . . . . . . . 59

4.3.1 Analysis of a 2-node network . . . . . . . . . . . . . . . . 60

4.3.2 Networks of arbitrary size . . . . . . . . . . . . . . . 66

4.3 .3 Simulation of a $64 \times 64$ network . . . . . . . . . . . . . . . 69

4.4 Summary . . . . . . . . . . . . . . . . . . . . . . 71

5 FLIP-Q: A QCIF resolution focal-plane array for low-power image processing 73

5.1 Introduction . . . . . . . . . . . . . . . . . . . . 73

5.2 Architecture . . . . . . . . . . . . . . . . . . . 74

5.3 Image processing implementation . . . . . . . . . . . . . . . . . . . . . . 75

5.3.1 Diffusion-based Gaussian filtering . . . . . . . . . . . . . . . . . . 77

5.3 .2 Block division control logic . . . . . . . . . . . . . . . . 80

5.3 .3 Energy computation . . . . . . . . . . . . . . . . 82

5.4 Readout circuitry . . . . . . . . . . . . . . . . . . . . . 84

5.4 .1 Reading control logic . . . . . . . . . . . . . . . . . 84

$5.4 .2 \mathrm{~A} / \mathrm{D}$ Conversion . . . . . . . . . . . . . . . . . . . 85

5.5 Experimental results . . . . . . . . . . . . . . . . . . . 86

5.5.1 Calibration of the time constant for diffusion . . . . . . . . . . 86

5.5 .2 Scale space . . . . . . . . . . . . . . . . . . . . . . . . . . . . . . . . . . 88

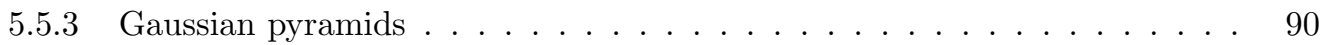

5.5.4 Multiresolution scene representation . . . . . . . . . . . . . . 91

5.5.5 Image pre-distortion for reduced kernel filtering . . . . . . . . . . . . . 91

5.5.6 Energy-based scene representation . . . . . . . . . . . . . . . . 93

5.5.7 Power consumption analysis . . . . . . . . . . . . . . . . . . . . . . . . . . . . 95

5.6 Comparative analysis . . . . . . . . . . . . . . . . . . . 97

5.7 Complementary information . . . . . . . . . . . . . . . . . 98

5.7 .1 Overview of the package . . . . . . . . . . . . . . 98

5.7 .2 Pin description . . . . . . . . . . . . . . . . . . 100

5.7 .2 .1 Pad types . . . . . . . . . . . . . . . 100

$5.7 .2 .2 \quad$ Pin map . . . . . . . . . . . . . . . . . . 102

5.7 .3 Programming guide . . . . . . . . . . . . . . . . . 111

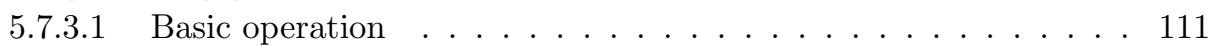

5.7.3.2 Processing primitives ................. 113

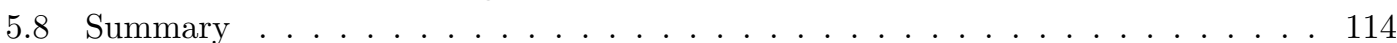

6 Wi-FLIP: A low-power vision-enabled WSN node 115

6.1 Imote2: system description . . . . . . . . . . . . . . . . 115

6.2 Interconnection plan . . . . . . . . . . . . . . . . . . . 118

6.3 Development board . . . . . . . . . . . . . . . . . . . . . . 119

6.4 Wi-FLIP programming . . . . . . . . . . . . . . . . . 119

6.5 Experimental tests . . . . . . . . . . . . . . . . . . . 122 
6.5.1 FLIP-Q processing primitives . . . . . . . . . . . . . 122

6.5.2 Exposure time control algorithm . . . . . . . . . . . . . . . . 123

6.5.3 Edge detection algorithm . . . . . . . . . . . . . . . 127

6.6 Comparison with the state of the art . . . . . . . . . . . . . . . . . . . 127

6.7 Summary . . . . . . . . . . . . . . . . . . . 130

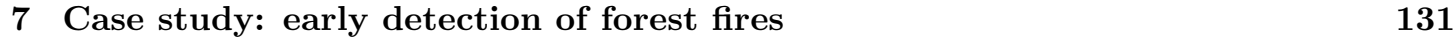

7.1 Motivations and background . . . . . . . . . . . . . . . . 131

7.2 Forest fire detection: state of the art . . . . . . . . . . . . . . . . . . . 132

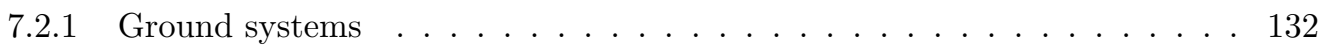

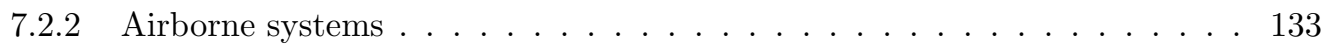

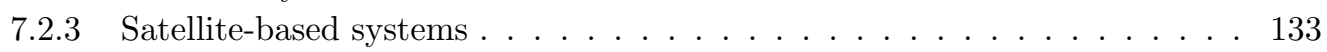

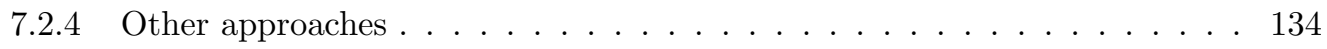

7.3 Vision-enabled WSN for early detection of forest fires . . . . . . . . . . . . . . 134

7.4 A vision algorithm for smoke detection . . . . . . . . . . . . . . . . . . . . . . . . . . . . . . . . . . . . .

7.5 Algorithm setting and preliminary tests . . . . . . . . . . . . . . . 140

7.5 .1 Parameter setting . . . . . . . . . . . . . . . . . . 142

7.5 .2 Results of the tests . . . . . . . . . . . . . . . . . . 145

7.6 On-site smoke detection . . . . . . . . . . . . . . . . . . . . . . 145

7.7 Tests with Wi-FLIP $\ldots \ldots \ldots \ldots \ldots \ldots \ldots$

7.8 Summary . . . . . . . . . . . . . . . . . . . . 151

Bibliography 153 



\section{List of Figures}

2.1 Sketch of a multi-cluster WSN. . . . . . . . . . . . . . . . . . 6

2.2 Top view (left) and bottom view (right) of the Imote2 platform. . . . . . . . . . 6

2.3 Basic interconnection sketch of the Cyclops platform and a snapshot of the system (with permission of the authors). . . . . . . . . . . . . .

2.4 Simplified interconnection scheme of the Imote2-based platform and a snapshot of the system (with permission of the authors). . . . . . . . . . . . .

2.5 Block diagram of the MeshEye $e^{\mathrm{TM}}$ platform along with a snapshot of the system (with permission of the authors). . . . . . . . . . . . . . .

2.6 Interconnection scheme of WiCa system and snapshot of the platform (with permission of the authors). . . . . . . . . . . . . . . .

2.7 Block diagram of the FireFly Mosaic platform along with a snapshot of the whole system (with permission of the authors). . . . . . . . . . . .

2.8 Block diagram of the CITRIC platform along with a snapshot of the whole system (with permission of the authors). . . . . . . . . . . . . .

2.9 Block diagram of EyeRIS ${ }^{\mathrm{TM}}$-based WSN node and snapshot of the platform (with permission of the authors). . . . . . . . . . . . . . . . . . 17

2.10 Basic sketch of the vision-enabled WSN node proposed. . . . . . . . . . . . . . 20

3.1 Diffusion-based spatial Gaussian filters with increasing $\sigma$ represented in the Fourier domain. . . . . . . . . . . . . . . . . 26

3.2 Diffusion-based spatially-discretized Gaussian filters with increasing $t$ represented over the discrete Fourier plane (overhead view) . . . . . . . . . . . . 27

3.3 Scale-space representation ( 5 scales) of the $512 \times 512$-px Lena image. . . . . . . . 28

3.4 Gaussian pyramid associated with the scale-space representation of Fig. 3.3. . . . 29

3.5 Example of dynamic image plane division: (a) Regular division; (b) Blocks of interest detected; (c) Gradual image plane division with maximum granularity in the ROI; (d) Abrupt image plane division with maximum granularity in the ROI and minimum for the rest. . . . . . . . . . . . . . . . . .

3.6 Examples of regular multiresolution representation: (a) Original Lena image; (b) Regular 4×4-px division; (c) Regular 16×16-px division. . . . . . . . . . .

3.7 Examples of foveation: (a) Application of a gradual division scheme like that of Fig. 3.5(c); (b) Corresponding ideal gradual foveation; (c) Application of an abrupt division grid like that of Fig. 3.5(d); (d) Corresponding ideal abrupt foveation. .

3.8 Multiresolusion filtering: (a) Image plane division into $2 \times 2$-px blocks; (b) Averaging within every block; (c) Redefinition of the division grid; (d) Averaging with the new configuration. . . . . . . . . . . . . . . . . .

3.9 Multiresolution filtering: (a) Grid division of Fig. 3.8(d) shifted back to its original position; (b) Averaging within every block in order to achieve binomial filtering.

3.10 Example of energy-based simplification of a scene: (a) Original image containing a flock of birds; (b) Block-wise division; (c) Result of computing Eq. (3.25) within every block and normalizing. . . . . . . . . . . . . . .

4.1 Processing node of a CNN implementing unconstrained linear diffusion . . . . . . 
4.2 Bidimensional CNN composed by $M \times N$ cells carrying out unconstrained linear diffusion . . . . . . . . . . . . . . . . . . . . 42

4.3 Eigenvalues for several ideal square CNNs implementing unconstrained diffusion 44

4.4 2-cell CNN programmed for unconstrained diffusion . . . . . . . . . . . . . . . 44

4.5 Largest real part of eigenvalues for several CNNs implementing unconstrained diffusion and suffering a $\sigma=10 \%$ mismatch . . . . . . . . . . . . . .

4.6 Ideal CNN implementing unconstrained linear diffusion: state variables at different time instants along the diffusion . . . . . . . . . . . . .

4.7 CNN implementing unconstrained linear diffusion with $\sigma=1 \%$ : state variables at successive time instants . . . . . . . . . . . . . . . . . . 47

4.8 Evolution of the RMSE with respect to the ideal unconstrained diffusion $(\tau=1 \mathrm{~ms}) 48$

4.9 Generic resistive grid . . . . . . . . . . . . . . . . . . . 49

4.10 Elementary resistive grid with (a) $\lambda \neq 0$ and (b) resulting RC network for $\lambda=0 \quad 50$

4.11 2-cell CNN programmed for constrained diffusion . . . . . . . . . . . . . 51

4.12 Ideal constrained diffusion: steady state for $\lambda=0.25, \lambda=0.5, \lambda=0.75$ and $\lambda=1$

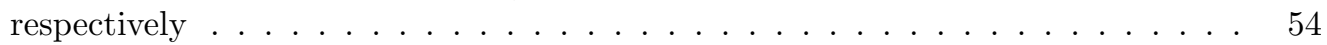

4.13 Constrained diffusion: steady state for $\lambda=0.1$ and $\lambda=0.3$ respectively with

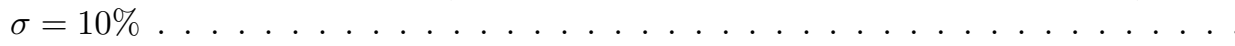

4.14 Constrained diffusion: steady state for $\lambda=0.5, \lambda=0.75, \lambda=1$ and $\lambda=2$ respectively with $\sigma=10 \% \ldots \ldots \ldots \ldots$

4.15 RMSE with respect to the ideal constrained diffusion . . . . . . . . . . . . . .

4.16 RMSE with respect to the ideal case for $\lambda=0.1 \Leftrightarrow t_{e q}=6.997 \tau, \lambda=0.3 \Leftrightarrow$ $t_{e q}=2.229 \tau$ and $\lambda=0.5 \Leftrightarrow t_{e q}=1.327 \tau$ respectively . . . . . . . . . . . .

4.17 RMSE with respect to the ideal case for $\lambda=0.75 \Leftrightarrow t_{e q}=0.877 \tau, \lambda=1 \Leftrightarrow t_{e q}=$ $0.665 \tau$ and $\lambda=2 \Leftrightarrow t_{e q}=0.362 \tau$ respectively . . . . . . . . . . . . .

4.18 Node for unconstrained diffusion in (a) a transconductor-based CNN implementation and (b) an RC network. . . . . . . . . . . . . . . . . .

4.19 Node for constrained diffusion in (a) a transconductor-based CNN implementation and (b) a resistive grid. . . . . . . . . . . . . . . . .

4.20 RC network performing linear diffusion (a) and its MOS-based counterpart (b) .

4.21 2-node ideal RC network (a) and its MOS-based implementation (b) . . . . . . .

4.22 Probability density function for the initial voltage (a) and for the sum of any two initial voltages (b) at the nodes of a RC network. . . . . . . . . . . . .

4.23 Probability density function for the final voltage (a) and for the sum of any two final voltages (b) at the nodes of a $\mathrm{RC}$ network. . . . . . . . . . . . .

4.24 Comparison between a MOS-based RC network and its ideal counterpart: (a) Original image, (b) MOS-diffused image at the instant of maximum error, (c) image diffused by the corresponding ideal RC network, (d) absolute error normalized to maximum individual pixel error . . . . . . . . . . . . . . . . . . .

4.25 RMSE of the MOS-based grid state vs. ideal RC grid state: (a) w/o mismatch, (b) Monte Carlo with $10 \%$ mismatch . . . . . . . . . . . . . . . . .

5.1 Floorplan of the prototype chip. . . . . . . . . . . . . . . . . . . 75

5.2 General view and microphotographs of the $F L I P-Q$ prototype. . . . . . . . . 76

5.3 Elementary cell of the array. . . . . . . . . . . . . . . . . . 77

5.4 Timing diagram of the operation of the elementary cell. . . . . . . . . . . . . 78

5.5 Diffusion control circuitry: (a) 15-stage inverter ring VCO and (b) diffusion control

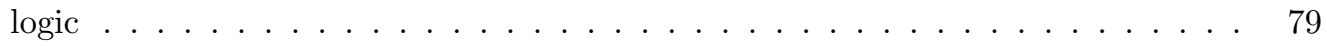

5.6 Column-wise focal-plane division control. . . . . . . . . . . . . . . . . 80

5.7 Row-wise focal-plane division control. . . . . . . . . . . . . . . . . . . . . 81

5.8 Generation of the signals for energy computation. . . . . . . . . . . . . . . . . . 84

5.9 Circuitry for column reading. . . . . . . . . . . . . . . . . . . . . . . 84

5.10 Circuitry for row reading. . . . . . . . . . . . . . . . . . 85

5.11 Symbol for the A/D Converter. . . . . . . . . . . . . . . . 86 
5.12 Laboratory setup for prototype testing. . . . . . . . . . . . . . . . . 87

5.13 Characterization of the four followers involved in the calibration of $\tau$. . . . . 87

5.14 Calibration of $\tau$ at the upper left corner (a) and upper right corner (b). . . . . . 88

5.15 RMSE for the on-chip scale space with respect to the ideal case . . . . . . . . . 89

5.16 Scale spaces along time. The first row corresponds to the on-chip scale space, the second one corresponds to the ideal scale space and finally the third one corresponds to their normalized difference. . . . . . . . . . . . . . . . . . .

5.17 Pyramid representation of two on-chip scale spaces. . . . . . . . . . . . . . . . 91

5.18 Examples of multiresolution scene representation . . . . . . . . . . . . . . . . 92

5.19 Results obtained by applying $\mathbf{G}_{x}$ and $\mathbf{G}_{y}$ to (a) 'Lena' and (b) 'Baboon' images captured by the chip, and $\mathbf{G}_{x}^{\prime}$ and $\mathbf{G}_{y}^{\prime}$ to the on-chip pre-distorted versions. The kernels $\mathbf{G}_{x}, \mathbf{G}_{y}, \mathbf{G}_{x}^{\prime}$ and $\mathbf{G}_{y}^{\prime}$ are defined in Eq. (3.20) . . . . . . . . . . . .

5.20 Results obtained by applying $\mathbf{G}_{b}$ to (a) 'Lena' and (b) 'Baboon' images captured by the chip, and $\mathbf{G}_{b}^{\prime}$ to the on-chip pre-distorted versions. Both $\mathbf{G}_{b}$ and $\mathbf{G}_{b}^{\prime}$ are

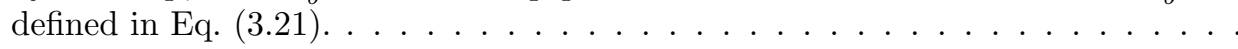

5.21 Captured image (a) and $\downarrow 2$ downsampled version (b) directly downloaded from the chip. . . . . . . . . . . . . . . . . . . .

5.22 On-chip filtering (first row), ideal off-chip filtering (second row) and normalized

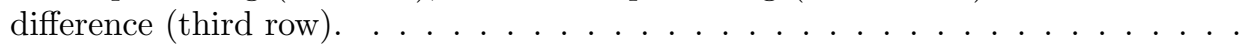

5.23 Examples of energy-based scene representation. . . . . . . . . . . . . . .

5.24 On-chip abrupt foveation around the blocks segmented by the computation of the energy in the second scene of Fig. 5.23 . . . . . . . . . . . . . . . . . 96

5.25 PGA-100 package . . . . . . . . . . . . . . . . . 100

5.26 PGA-100 grid-cavity interconnection plan . . . . . . . . . . . . . . . . 100

5.27 Bonding diagram of the chip for a ceramic 100-pin grid array. . . . . . . . . . 101

5.28 Pin map of the packaged chip (BOTTOM VIEW) . . . . . . . . . . . . 103

5.29 Pin map of the packaged chip (TOP VIEW) . . . . . . . . . . . . . . . . 104

5.30 Loading process of the bit string ' $1010 \ldots 10$ ' at the focal-plane division registers. The pattern is repeated until loading all the bits into the registers. . . . . . . . 111

5.31 Example of a loading process of the reading registers. . . . . . . . . . . . . . . 112

5.32 Timing diagram of the A/D converter operation. . . . . . . . . . . . . . . . 112

5.33 Loading the bit string '110000000000' at the diffusion control register with external clocking. . . . . . . . . . . . . . . . . . . 113

5.34 Reading out the bit string ' 110000000000 ' at the diffusion control register by using the internal VCO. . . . . . . . . . . . . . . . . . . 113

5.35 Generation of the signals for block-wise energy computation. . . . . . . . . . . 114

6.1 Top and bottom view of Imote2 along with its block diagram. . . . . . . . . . . . 116

6.2 Connectors comprising the "advanced sensor board" interface of Imote2. . . . . . 117

6.3 Wi-FLIP PCB: Schematic and layout. . . . . . . . . . . . . . . . . 120

6.4 Wi-FLIP: a vision-enabled node for wireless applications. . . . . . . . . . . . . 121

6.5 Imote2 Interface Board IIB2400 (a) and base station MIB520 (b): two different ways of retrieving information from Wi-FLIP. . . . . . . . . . . . . 123

6.6 Scale space generation in Wi-FLIP. The diffusion is internally controlled as explained in Section 5.3.1. . . . . . . . . . . . . . . . . 123

6.7 Example of multiresolution representation in Wi-FLIP. . . . . . . . . . . . . 123

6.8 Salient region estimation in Wi-FLIP. . . . . . . . . . . . . . . . . . . 124

6.9 Flowchart of the exposure time control algorithm. . . . . . . . . . . . . . 125

6.10 Scene surveyed while running the exposure time control algorithm. . . . . . . . . 127

6.11 Flowchart of the edge detection algorithm. . . . . . . . . . . . . . . . . 128

6.12 Two frames after running the edge detection algorithm. . . . . . . . . . . . . . 129

7.1 Sketch of the proposed system . . . . . . . . . . . . . . . . 135 
7.2 Intensity histogram of the RGB components and luminance of a vegetation zone without smoke and in presence of smoke. . . . . . . . . . . . . . . 137

7.3 Intensity histogram of the RGB components and luminance of a vegetation zone without smoke and in presence of smoke. . . . . . . . . . . . 138

7.4 Flowchart of the smoke detection algorithm. . . . . . . . . . . . . . . . . . . 141

7.5 Number of smoke pixels per frame for each of the 9 different sequences analyzed. 143

7.6 Average variation of the number of pixels affected by smoke for each sequence. . 143

7.7 Normalized average increase of the pixels affected by smoke. . . . . . . . . . . . . 144

7.8 Representation of $N(t)$ for each of the 9 different sequences analyzed. . . . . . . 144

7.9 Representation of $Z(t)$ for the sequences analyzed. . . . . . . . . . . . . . . 145

7.10 Normalized dynamics (\%) of all the regions composing the image plane division for three recordings until detecting smoke. . . . . . . . . . . . . . 146

7.11 Forest debris burnt during the field tests. . . . . . . . . . . . . . . . . . 147

7.12 Arrangement of the EyeRIS ${ }^{\mathrm{TM}}$ system (left at both photos) and the commercial camcorder (right at both photos) during the field tests. . . . . . . . . . . . 148

7.13 Consecutive frames captured by the EyeRIS ${ }^{\mathrm{TM}}$ (first row) and their corresponding candidate bins (second row) during the first controlled burn. . . . . . . . . . . . 148

7.14 Evolution of $N(t)$ and $Z(t)$ for the first and second burn respectively. . . . . . 148

7.15 Smoke segmentation and alarm image from Wi-FLIP. . . . . . . . . . . . 149

7.16 Snapshots taken during the latest test carried out. . . . . . . . . . . . . . . 150

7.17 Images sent via radio by Wi-FLIP for two of the alarms triggered. . . . . . . . . 151 


\section{List of Tables}

2.1 Comparison of vision-enabled WSN platforms . . . . . . . . . . . . . . . . 19

4.1 Equivalences between constrained and time-controlled unconstrained linear diffusion 57

4.2 Numerical verification of the approximations realized. . . . . . . . . . . . 67

5.1 Summary of the prototype chip features. . . . . . . . . . . . . . . . 76

5.2 Summary of the A/D converter. . . . . . . . . . . . . . . 86

5.3 Power consumption of the chip for different focal-plane configurations. . . . . . . 97

5.4 Comparison of focal-plane processing chips performance. . . . . . . . . . . . . . 99

5.5 Pad types employed in the prototype. . . . . . . . . . . . . . . 102

5.6 Pin assignment of the prototype: pins from 1 to $20 . \ldots \ldots$. . . . . . . . . 106

5.7 Pin assignment of the prototype: pins from 21 to $40 \ldots \ldots$. . . . . . . . . . 107

5.8 Pin assignment of the prototype: pins from 41 to $60 \ldots \ldots$. . . . . . . . . 108

5.9 Pin assignment of the prototype: pins from 61 to $80 \ldots \ldots$. . . . . . . . . 109

5.10 Pin assignment of the prototype: pins from 81 to 100 . . . . . . . . . . . . 110

6.1 Summary of the main operating parameters of Imote2. . . . . . . . . . . . . . . 117

6.2 FLIPQ-to-Imote2 interconnection plan. . . . . . . . . . . . . . . 118

6.3 Performance of $W i$-FLIP running the exposure time control algorithm. . . . . . . 126

6.4 Performance of $W i-F L I P$ running the edge detection algorithm. . . . . . . . . . . 129

6.5 Comparison of the imager's performance for different vision-enabled WSN nodes reported in the literature. . . . . . . . . . . . . . . . . . . 130

7.1 Normalized average increase, referred to the background, suffered by each component of the pixels within the zones marked in Fig. 7.2 and Fig. 7.3, when smoke

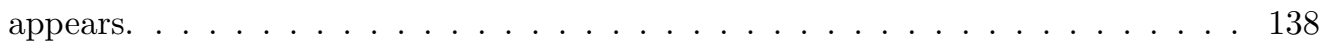

7.2 Summary of the algorithm setting. . . . . . . . . . . . . . . . 146 



\section{Abbreviations}

$\begin{array}{ll}\text { ARM } & \text { Advanced RISC Machine } \\ \text { CMOS } & \text { Complementary Metal Oxide Semiconductor } \\ \text { CNN } & \text { Cellular Nonlinear Network } \\ \text { CPLD } & \text { Complex Programmable Logic Device } \\ \text { DFT } & \text { Discrete Fourier Transform } \\ \text { DNL } & \text { Differential Non-Linearity } \\ \text { ENOB } & \text { Effective Number Of Bits } \\ \text { FFT } & \text { Fast Fourier Transform } \\ \text { FOM } & \text { Figure Of Merit } \\ \text { GIS } & \text { Geographic Information System } \\ \text { GPIO } & \text { General Purpose Input/Output } \\ \text { INL } & \text { Integral Non-Linearity } \\ \text { LEO } & \text { Low Earth Orbit } \\ \text { LIDAR } & \text { LIght Detection And Ranging } \\ \text { MAC } & \text { Multiple-Accumulate Operation } \\ \text { MCU } & \text { MicroController Unit } \\ \text { MMC } & \text { MultiMedia Card } \\ \text { PAL } & \text { Phase Alternating Line } \\ \text { PCB } & \text { Printed Circuit Board } \\ \text { PDE } & \text { Partial Differential Equation } \\ \text { PE } & \text { Processing Element }\end{array}$


PMIC Power Management Integrated Circuit

PWM Pulse Width Modulator

QCIF Quarter Common Intermediate Format

RISC Reduced Instruction Set Computer

ROI Region Of Interest

SAR Succesive Approximation Register

SIMD Single Instruction Multiple Data

SMA SubMinituare version A

SRAM Static Random Access Memory

UART Universal Asynchronous Receiver-Transmitter

UAV Unmanned Aerial Vehicles

VGA Video Graphics Array

VLSI Very Large Scale Integration

VCO Voltage-Controlled Oscillator

WSN Wireless Sensor Network 


\section{Chapter 1}

\section{Introduction}

\subsection{Ubiquitous computing: a new era in computer history}

It has been roughly 60 years since the first mainframe was devised [Eckert \& Mauchly, 1947], starting the first generation of computing systems. During this period, extremely short if we consider the time it took mankind to accomplish such milestone, technological advances have succeeded at an incredible pace, mainly thanks to the rapid evolution of computers. This evolution has been in turn greatly supported by the density of transistors available to be integrated in circuits, which has been steadily increasing according to Moore's Law ${ }^{1}$ [Moore, 1965]. Chips have been getting smaller and cheaper while their performance has been improving. Thus, it took less than 30 years to put directly at hands of individuals processing power unthinkable few years ago. Personal computers meant another landmark [Green, 1975], the second generation of computing systems. People got used to the presence of computers everywhere. First laptops came on stage [Osborne, 1981], opening new possibilities of taking advantage of the computational capabilities at disposal. In addition, computers started to be interconnected, locally at first, progressively wider later. Under these circumstances, it was M. Weiser ${ }^{2}$ who first coined the term ubiquitous computing [Weiser, 1991] to refer to a new era in computing systems, the third generation. This new paradigm would be based on the seamless integration of computers in the background of daily people's life. A natural interaction among users and surrounding devices falls at the heart of such integration. These devices must be sensitive, adaptive and responsive to the presence of human activity. Moreover, they must be so embedded in the environment that we make use of them without even thinking about them.

\footnotetext{
${ }^{1}$ Gordon E. Moore, retired now, is the co-founder and former Chairman and CEO of Intel Corporation. He revised the original formulation of his famous Law in 1975, stating that the density of transistors would double not every year, as he first predicted in 1965, but every two years.

${ }^{2}$ Mark Waiser was the CTO at Xerox's Palo Alto Research Center (PARC). He is often referred to as the father of ubiquitous computing. He passed away in 1999.
} 


\subsection{Wireless Sensor Networks: a basic building block}

The list of technological challenges underlying this new era in computer history is, even now, 20 years since its first definition, almost endless. Nevertheless, advances in key issues have been accomplished. Among them, Wireless Sensor Networks (WSNs) play a remarkable role. Tiny low-cost autonomous sensor nodes endowed with processing and communication capabilities are the backbone of WSNs [Pottie \& Kaiser, 2000]. Their development has been closely linked to advances in embedded processors [PXA271 (Marvell)], operating systems [Levis \& Gay, 2009] and wireless communication [IEEE Std 802.15.4, 2006], providing sensors with very high power efficiency. The significance of WSNs within the paradigm of ubiquitous computing can be better understood through two references. In 1999, BusinessWeek ${ }^{3}$ chose networked microsensors as one of the 21 most important technologies for the 21st century [Gross, 1999]. In 2003, Technology Review ${ }^{4}$ heralded WSNs as one of the 10 emerging technologies that will change the world [Wade, 2003].

The potential of application of WSNs has been jointly growing with the sensing capabilities incorporated into the nodes. Thus, it is the possibility of measuring magnitudes such as temperature, humidity and barometric pressure what allows for addressing wildfire monitoring [Doolin \& Sitar, 2005; Son et al., 2006; Hefeeda, 2007]. Humidity and temperature also permit to implement precision agriculture [Langendoen et al., 2006; McCulloch et al., 2008]. By additionally sensing wind speed and direction, low-cost fine-grained meteorological station networks can be achieved [Barrenetxea et al., 2008]. Sensors of photosynthetically active radiation (PAR) help to study forest canopy [Tolle et al., 2005] whereas seismoacoustic sensing supports volcanic studies [Werner-Allen et al., 2006]. These examples show that the larger the catalog of sensing capabilities implemented at the nodes is, the wider the scope of application WSNs take on.

\subsection{Vision: a step forward in WSNs}

Recently, a new possibility concerning WSNs has begun to be explored by the research community: multimedia sensing [Eren \& Akan, 2005; Akyildiz et al., 2007]. Specifically, the incorporation of vision results of great interest. To start with, it would mean a revolution in the field of surveillance systems [Cucchiara, 2005]. Pan-tilt-zoom (PTZ) cameras surveying large areas could be substituted by distributed smart vision sensors capable of on-site decision making. In security applications, different points of view of the same scene could be easily monitored, overcoming occlusion problems and making the ultimate goal of the network - object detection, face

\footnotetext{
${ }^{3}$ Business Week is a weekly business magazine published by Bloomberg L.P. It was first published in September 1929. It is considered one of the most influential business magazines in USA.

${ }^{4}$ Technology Review is the oldest technology magazine in the world (est. 1899). It is published by Technology Review Inc., an independent media company owned by the Massachusetts Institute of Technology.
} 
recognition etc. - more reliable and robust. Similarly, current WSN applications would take a new dimension. Two clear examples are wildfire monitoring and precision agriculture. In the first case, image processing would permit not only to monitor a certain area from the perspective of environmental conditions, but also to carry out visual inspection in order to perform early detection of smoke or flames. In the second case, scene analysis would provide sensors with the chance of detecting the presence of plagues or diseases, thus preventing crops from ruining.

But the implementation of vision hardware at WSN nodes is not a trivial issue at all. While most of the above mentioned sensing capabilities are related to scalar physical magnitudes, visual stimulus implies to handle a massive flow of multidimensional information. Taking into account the very strict power budgets allocated to the nodes, simply the capture and digitization of an image sequence could represent a significant percentage of their energy consumption. But the critical point arises just afterwards. On one hand, the sequence could be simply transmitted for remote processing, affecting dramatically the scalability and bandwidth of the network. On the other hand, the node itself could deal with the image sequence by taking advantage of its processing capabilities. In this case, the nature of such processing is greatly influenced by the energy constraints, demanding new strategies which permit to reach the targeted result with the minimum possible power cost.

It is precisely on this demand for new strategies enabling local image processing at WSN nodes where this book focuses. After reviewing the state of the art about vision-enabled nodes, we will propose a non-conventional architectural approach for the sake of energy efficiency. This approach, rooted in the processing scheme of vertebrates' visual system, is based on a hierarchical division of the processing chain: most demanding computational tasks, consisting of a very regular computational flow applied over a large amount of raw image data, are conveyed to the focal plane. There, distributed processing elements (PEs), concurrent with the photosensitive devices, influence the image capture and generate a pre-processed representation of the scene that removes redundant information for subsequent digital stages. This simplified data flow will allow, for example, to lower the clock speed of the embedded processor for a prescribed frame rate, what is a key issue when it comes to reduce power consumption [Rabaey, 1995]. Furthermore, the focal-plane operators are implemented by power-efficient analog building blocks, which may individually be a little imprecise, but accurate enough as a collective to render the targeted image processing. In short, highly demanding computational tasks are implemented by an aggregate of simple elements with moderate accuracy, but very efficiently in terms of the energy required to realize a single operation. Thus, we report a prototype vision chip with QCIF resolution $(176 \times 144 \mathrm{px})$ based on this processing scheme. This prototype, tested and fully functional, delivers a pixel-by-pixel digital output as a result of reconfigurable focal-plane analog processing and subsequent on-chip A/D conversion. The ultra low-power operation achieved have permitted its integration with a commercial WSN platform, demonstrating the suitability of the processing 
architecture proposed to incorporate vision into WSNs in a very efficient way. Finally, a case study is presented: forest fire smoke detection by vision-enabled WSNs. A vision algorithm encoding the spatio-temporal dynamics of smoke showing up against a vegetation background has been devised. It makes use of a subset of the focal-plane primitives implemented in the prototype. The robustness of this vision algorithm with respect to false alarms has been successfully checked out in real field tests consisting of controlled burnings of forest debris. 


\section{Chapter 2}

\section{Vision-enabled WSN nodes: state of the art}

\subsection{A brief introduction to WSNs}

A Wireless Sensor Network can be defined as a set of autonomous sensor nodes which are densely and wirelessly deployed throughout a region of interest [Akyildiz et al., 2002]. Each node, commonly referred to as a mote, includes sensing, data processing and communicating components. Its usual workflow consists of sensing data, processing it locally and communicating only the required information resulting from this processing. A remarkable feature is that the exact location of the nodes comprising the network does not need to be predetermined. Communication protocols and algorithms possess self-organizing capabilities. Typically, sensor nodes will be clustered. Within each cluster, a node acts as the head, routing data coming from the rest of sensors to a specialized node called sink, or base station. Multi-hop wireless communication is used throughout this process. End users can retrieve data and reconfigure the network through the base station. A generic sketch of a multi-cluster WSN is depicted in Fig. 2.1.

Actual deployments of WSNs usually implement one of three general configurations [Kulkarni et al., 2010], namely: periodic reporting, event detection, and database-like storage. Periodic reporting is by far the most used application scenario: nodes sample regularly their environment, process the sensory data and store the result to be eventually sent to the base station. In event detection, nodes sense their environment and evaluate the data immediately for its usefulness. If an event of interest is detected, it is notified at once. In database-like storage systems, all sensory data (periodic sampling and/or events) is stored locally in the nodes. End users, by means of the base stations, search for interesting data and retrieves it directly from the corresponding nodes. 
Networks, once deployed, must be able to collect data uninterruptedly for weeks, months or even years. Since energy consumption determines the node lifetime, sensors have limited computational and communication resources. They include lightweight embedded processors with a few MBytes of RAM. These processors usually run at 10-100 MHz at most, rather than typical GHz in current 64-bit CPUs. Their low-power radios can send to a few hundreds of kbps, rather than 802.11's tens of Mbits. As a result, software needs to be very efficient in terms of both CPU cycles and memory use. Consider Fig. 2.2 as an example. It shows a commercial WSN node, the Imote2 platform [Imote2 (MEMSIC)]. This node is built around a 32-bit ARM5 processor [PXA271 (Marvell)] which can operate in a low voltage $(0.85 \mathrm{~V})$, low frequency $(13 \mathrm{MHz})$ mode, hence enabling very low power operation. The PXA271 is really a multi-chip module which also includes 256kB SRAM, 32MB SDRAM and 32MB of FLASH memory. An 802.15.4-compliant radio transceiver [CC2420 (Texas Instr.)] is also integrated into the Imote2 system. Note that external sensor boards can be connected through expansion connectors. In fact, the prototype vision chip reported in this work has been interconnected to the Imote2 platform by using these connectors, as thoroughly described in Chapter 6.

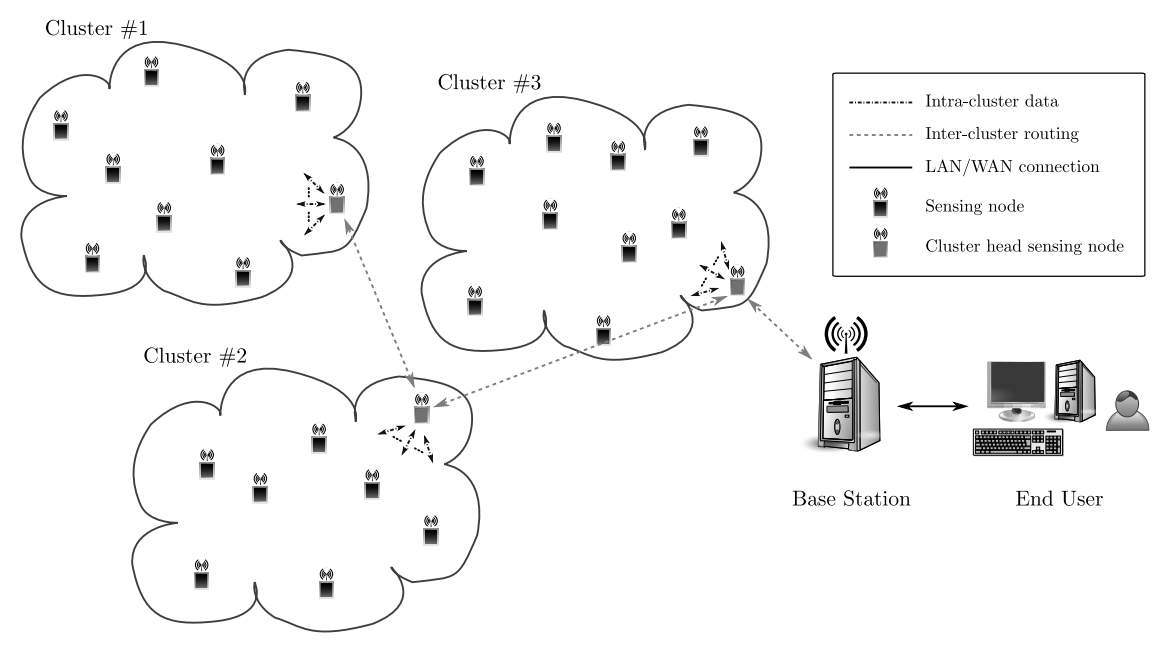

FigURE 2.1: Sketch of a multi-cluster WSN.

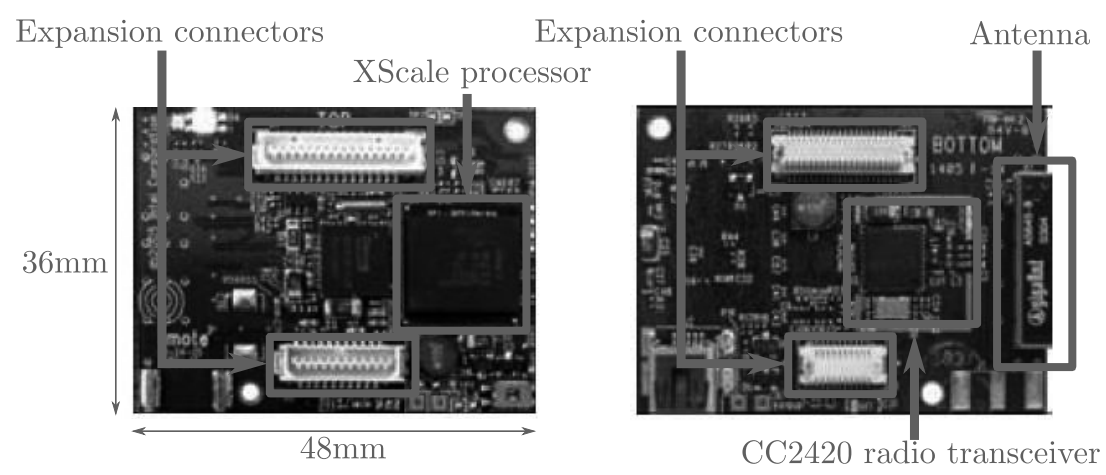

Figure 2.2: Top view (left) and bottom view (right) of the Imote2 platform. 

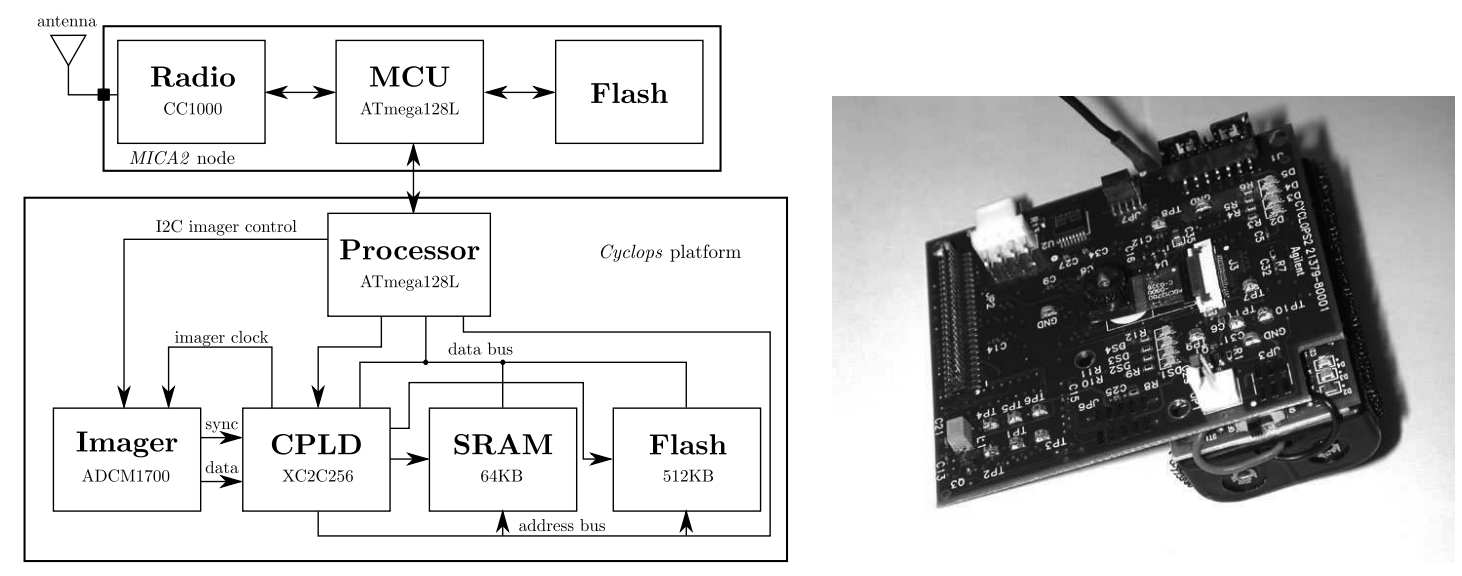

Figure 2.3: Basic interconnection sketch of the Cyclops platform and a snapshot of the system (with permission of the authors).

\subsection{A survey on vision-enabled WSN nodes}

\subsubsection{Cyclops [Rahimi et al., 2005]}

The term Cyclops refers to an electronic interface between an off-the-shelf camera module and a lightweight wireless host. The philosophy behind this approach is to separate the computational resources used for imaging from those ones assigned to communication. Thus, Cyclops consists of an 8-bit RISC processor [ATmega128L (ATMEL)], a CPLD, external SRAM (64KB) and external FLASH $(512 \mathrm{~KB})$. The camera module is an ultra compact CIF resolution $(352 \times 288 \mathrm{px})$ CMOS imager [ADCM-1700 (Agilent)]. The processor controls the communication with a wireless host [MICA2 (MEMSIC)], as well as the different parameters of the imager for frame capture. The CPLD provides high speed clock, synchronization and memory control. Both processor and CPLD can perform local computations on the image to produce an inference. The camera module contains a complete image processing pipeline for demosaicing, image size scaling, color correction, tone correction and color space conversion. It also implements automatic exposure control and automatic white balance. A basic sketch of the Cyclops platform and the interconnection of the different modules is depicted in Fig. 2.3 along with a snapshot of the system, reproduced here with permission of the authors. Note that the camera is embedded into the same PCB than the rest of elements comprising Cyclops.

For the sake of transparency in using resources such as the imager or the SRAM, firmware has been written in nesC [Gay et al., 2003], a programming language tailored for TinyOS [Levis \& Gay, 2009]. One of the main advantages of having separated the computational resources for image processing (Cyclops) from those ones dedicated to wireless communication (MICA2) is flexibility to develop this firmware. The design principle is to support the long synchronous operations associated with image processing. Time-consuming computational blocks performed over 
serialized images can be implemented without considering possible starvation for asynchronous requirements of the network. From the opposite point of view, image processing is speeded up on not having to deal with such requirements.

The Cyclops platform presents different power states according to the resources required to perform a certain operation. The worst case corresponds to permanent writing memory access, with a power consumption of $64.8 \mathrm{~mW}$. For the image sensor, a typical consumption of $42 \mathrm{~mW}$ is reported. Finally, the MICA2's MCU presents a power consumption of $24 \mathrm{~mW}$. The radio module reaches $81 \mathrm{~mW}$ when transmitting with maximum power. Adding up all these figures, a simple application of capturing an image sequence, storing it and transmitting it would mean a power consumption of $211.8 \mathrm{~mW}$, assuming that all modules are working concurrently.

Regarding the frame rate, the values reported are low due to the significant latency introduced by the pipeline and firmware layers. For image sizes of $32 \times 32 \mathrm{px}, 64 \times 64 \mathrm{px}$ and $128 \times 128 \mathrm{px}$ and room light conditions, the maximum frame rate achievable is, respectively, 12.5fps, 11.1fps and 7.1fps. If some processing is realized, frame size affects almost linearly the additional time required to complete the whole computation. For example, Sobel derivativation or background subtraction can be carried out with maximum frame rates of, respectively, 11.8fps, 8.7fps and 4.3fps.

Finally, two applications are presented, namely, object detection and hand posture recognition. The key point in the development of these applications is the progressive data reduction along the flow of execution. To start with, images are kept with the minimum size which the application can afford. Besides, the algorithms arrange their computations in such a way that data is rapidly cut down. Additionally, results of previous computations are reused multiple times, saving clock cycles and memory. Thus, the object detection algorithm runs at 10.3fps, 6.6fps and 2.6fps for frame sizes of $32 \times 32 \mathrm{px}, 64 \times 64 \mathrm{px}$ and $128 \times 128 \mathrm{px}$, respectively. This algorithm constructs the stationary background by analyzing a certain number of images. Then, it generates a model of the foreground based on the absolute difference of the instantaneous image and the constructed background. Using a threshold filter and, subsequently, a blob filter, the object map is eventually obtained. The threshold value is constantly estimated as a factor of the background illumination. Regarding the hand posture recognition application, the algorithm runs below 2fps for a frame size of $64 \times 64 \mathrm{px}$. In this case, the algorithm tries to recognize static hand gestures. It transforms an image into a feature vector which is then compared to the feature vectors of a training set of gestures. This transformation is based on the calculation of the orientation histogram, which is in turn calculated by applying the Sobel derivative masks.

Therefore, as a summary, it can be said that the main feature of Cyclops is the total separation of the computational resources dedicated to image processing from those ones dedicated to network processing. This simplifies the development of firmware and speeds up the execution 

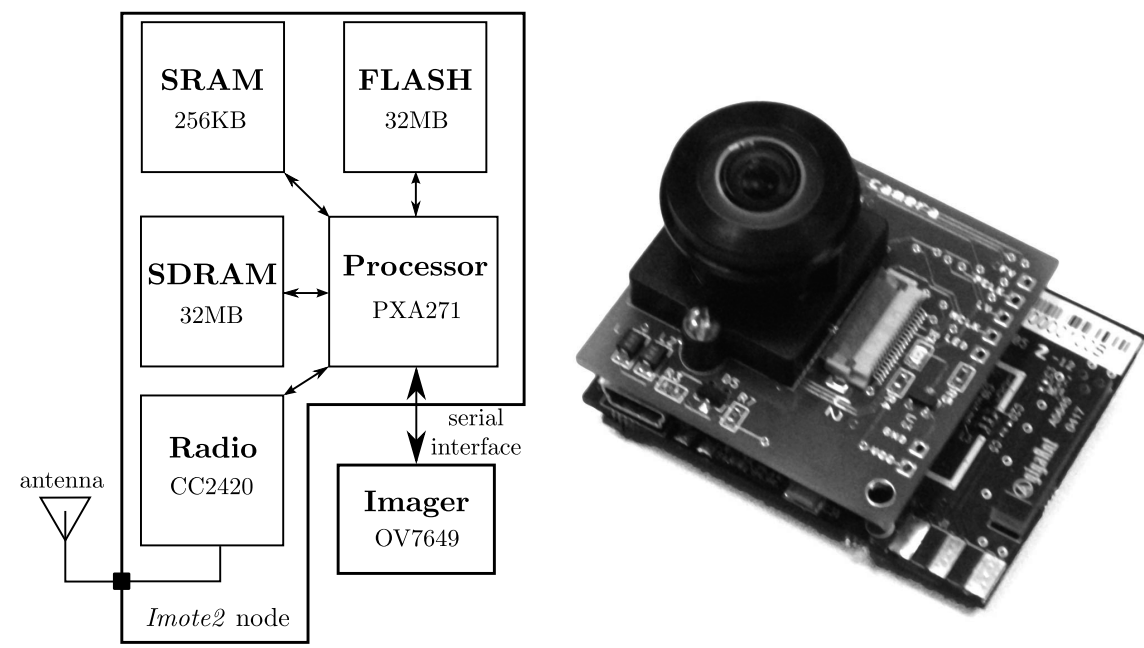

Figure 2.4: Simplified interconnection scheme of the Imote2-based platform and a snapshot of the system (with permission of the authors).

of vision algorithms. Concerning the strategies for energy efficiency, image processing is based on a constant data reduction and reuses computations in order to save clock cycles and memory accesses.

\subsubsection{Imote2-based platform [Teixeira et al., 2006]}

This vision-enabled WSN platform is quite simple. It consists of a direct interconnection between the Imote2 node, briefly described in Section 2.1, and a commercial CMOS imager [OV7649 (Omnivision)] through a serial interface. This imager can capture color VGA images $(640 \times 480 \mathrm{px})$ at $30 \mathrm{fps}$ and QVGA $(320 \times 240 \mathrm{px})$ at $60 \mathrm{fps}$ with a power consumption of $44 \mathrm{~mW}$. Automatic exposure control, gain control and white balance are additional functionalities available. In fully-active mode, with Imote2 working at $104 \mathrm{MHz}$ and a frame rate of $8 \mathrm{fps}$, the system consumes $322 \mathrm{~mW}$. A simplified interconnection scheme of this platform along with a snapshot of the system is depicted in Fig. 2.4.

An application to assisted living is presented [Teixeira \& Savvides, 2008]. Six Imote2-based platforms are placed at different points of the ceiling of a room. They are a single hop away from their base station in a star topology. The nodes track the presence of people by analyzing images downsampled to $80 \times 60 \mathrm{px}$ at around $14 \mathrm{fps}$ with the processor working at $208 \mathrm{MHz}$. Timestamped information is then forwarded from each node to the base station, which aggregates it and reports accordingly to a gateway computer. The ultimate objective is to recognize unsafe and out-of-the-ordinary behaviors of the people walking around the room.

Due to the simplicity of this WSN node, there are two major parameters to be adjusted in order to reduce power consumption. First of all, the speed of the processor. For the application 
reported, the processor works at $208 \mathrm{MHz}$. A slower operation mode is possible by setting the speed of the processor to $13 \mathrm{MHz}$. The other possibility is to reduce the image resolution as much as the application under consideration can afford.

\subsubsection{MeshEye ${ }^{\mathrm{TM}}[$ Hengstler et al., 2007]}

This wireless smart vision node is an extension of the work introduced in [Downes et al., 2006]. It is mostly oriented to applications within the field of distributed intelligent surveillance. Its design is characterized by the implementation of a direct interface between the embedded processor and several image sensors. No FPGA or CPLD device is used. The core of the system is a 32-bit ARM7 processor [AT91SAM7S (ATMEL)]. Up to eight kilopixel imagers can be hosted in the node. A VGA $(640 \times 480 \mathrm{px})$ camera module is also supported. The wireless connection to other nodes is established through an 802.15.4-compliant radio transceiver [CC2420 (Texas Instr.)]. Finally, a MMC/SD flash memory card provides sufficient non-volatile memory for temporary frame buffering and even image archival. A block diagram of the MeshEye ${ }^{\mathrm{TM}}$ system is depicted in Fig. 2.5 along with a snapshot of the system, reproduced here with permission of the authors. In a first implementation, two kilopixel imagers [ADNS-3060 (Agilent)] were used, with a VGA camera [ADCM-2700 (Agilent)] centered between them. Every kilopixel imager features a highspeed sensor with a resolution of $30 \times 30$ px outputting 6 -bit grayscale images at up to 50 fps. The VGA module can deliver grayscale or 24-bit color images. All these pixel arrays are placed in parallel and face the same direction, focusing to infinity. They work collaboratively in the following way. One of the kilopixel imagers is continuously polling for moving objects entering its field of view (FoV). Once one or possibly more objects have been detected, position and size are determined for each object. Basic stereo vision of the two kilopixel imagers yields the distance to the object. This information allows the calculation of the region of interest (ROI) containing the object within the VGA camera's image plane. Thus, the processor triggers this camera in order to capture a high-resolution grayscale or color ROI which includes the detected object. Finally, this ROI is processed according to the specific target of the application. The reason behind using kilopixel imagers for stereo vision instead of simply two VGA camera modules is to reduce energy consumption. Although downsampled frames could be delivered by these VGA modules in order to analyze kilopixel images, they still acquire the entire pixel array internally and downsample it digitally. Hence low-resolution frame capture with a kilopixel imager is more energy-efficient even if the power consumption per pixel is similar in both cases.

Image processing algorithms for object detection, stereo matching and object acquisition are presented. They are intentionally kept at low computational complexity in order to reach the corresponding application target at minimum energy cost. Object detection is performed over the left kilopixel imager through a very simple background subtraction on a frame-by-frame 


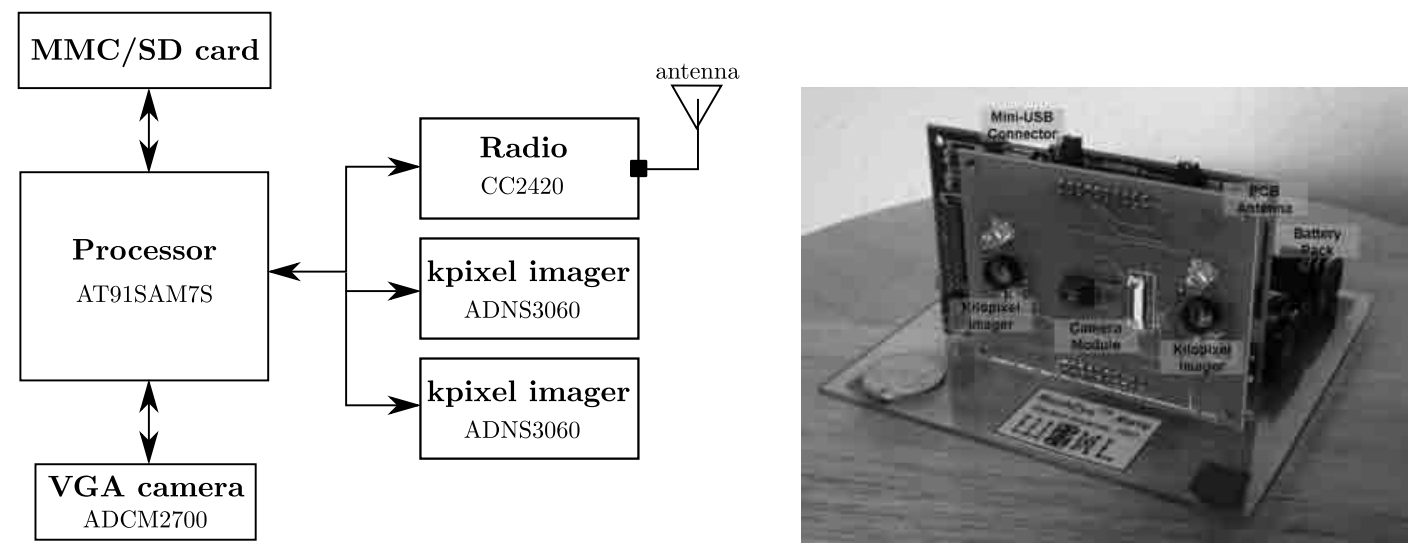

Figure 2.5: Block diagram of the MeshEye ${ }^{\mathrm{TM}}$ platform along with a snapshot of the system (with permission of the authors).

basis. Then, stereo matching correlates the result of this detection with the difference between the current frame and the background representation of the right kilopixel imager. If a positive match cannot be established, the left imager will continue detecting the object while it is within its FoV. This happens when the object lies outside the overlapping FoV of the two kilopixel imagers. Otherwise, knowing the object's position within both imagers and its size, the VGA camera module is triggered to acquire a high resolution snapshot of the object. A power model of this basic surveillance operation is reported. It also includes the power consumption of the radio transceiver communicating the result of intermediate level processing over the high resolution snapshot of the object. Two AA batteries power the whole system. Several frame rates below 2 fps are considered. As an example, the power consumption for 0.5 fps while no object is detected is about $7 \mathrm{~mW}$. When objects with a size of around $64 \times 64 \mathrm{px}$ show up within the scene being surveyed, the power consumption reaches around $155 \mathrm{~mW}$. Therefore, the activity within the scene governs the lifetime of the system. Frequent occurrences of objects reduce considerably its lifetime.

In a nutshell, MeshEye ${ }^{\mathrm{TM}}$ stands out for the direct interface between an embedded processor and vision devices. No FPGA or CPLD is included. Its design has been realized with a specific application in mind: distributed intelligent surveillance. For a basic surveillance task, it presents very competitive power consumption figures. However, its uptime is greatly influenced by the motion within the scene surveyed. Thus, MeshEye ${ }^{\mathrm{TM}}$ rapidly starves in scenes with much activity.

\subsubsection{WiCa [Kleihorst et al., 2007]}

Low-level image processing tasks [González \& Woods, 2002], also known as early vision tasks, are characterized by their regularity. They are equally defined for each pixel as a function of 
its own value and its immediate neighborhood. More importantly, the computation over each pixel is often independent from the result of the computations over the rest, opening the door for parallel processing. The $\mathrm{WiCa}$ platform takes advantage precisely of this potential parallel processing to reduce the number of memory accesses, clock speed and instruction decoding. The key component to achieve it is a high-performance digital processor called Xetal-II [Abbo et al., 2008]. This processor is designed ad-hoc for frame-based real-time video analysis. It is based on the SIMD (Single-Intruction Multiple-Data) paradigm [Unger, 1958], which fits perfectly with the characteristics of low-level image processing tasks just mentioned. Xetal-II incorporates 320 PEs which work in parallel to reach a measured peak performance of 107GOPS when operating at $84 \mathrm{MHz}$ and $1.2 \mathrm{~V}$, with a power consumption of $600 \mathrm{~mW}$, that is, around $5.6 \mathrm{~mW} / \mathrm{GOPS}$. This number of PEs, 320, is an integral divisor of the image lines for most standard video formats, what makes it ideal for parallel processing no matter the format finally chosen. The chip is manufactured in 90nm CMOS technology and occupies $74 \mathrm{~mm}^{2}$. It also integrates a $10 \mathrm{Mbit}$ memory allowing for energy-efficient inter-frame and intra-frame computations. The programmation of Xetal-II is carried out by using an extended C programming language called $\mathrm{XTC}$ whose major feature is the introduction of a vector data type to represent 320-element variables. In addition to Xetal-II, $\mathrm{WiCa}$ includes a general-purpose processor [ATMEL8051] for control and medium- and high-level image processing. Both processors are coupled using a dual port RAM controlled by a CPLD that enables them to work in a shared workspace on their own processing pace. Finally, a MMC/SD memory card provides extra non-volatile memory. For radio communication, an external module called AquisGrain [Espina et al., 2006] containing a transceiver [CC2420 (Texas Instr.)] is connected to WiCa. Regarding vision sensors, one or two off-the-shelf VGA color image sensors [OM6802 (Philips)] can be also connected to the platform. These sensors work at $30 \mathrm{fps}$ with digital RGB output. They can be also set at $60 \mathrm{fps}$ in CIF resolution. The interconnection scheme of $\mathrm{WiCa}$ is depicted in Fig. 2.6 together with a snapshot of the platform, reproduced with permission of the authors.

No direct application of this version of the WiCa platform is presented. However, by making use of a previous version [Kleihorst et al., 2006], which includes the precursor of Xetal-II processor, applications like Canny edge detection [Geelen et al., 2009] or real-time gesture recognition [Zivkovic et al., 2008] have been reported. In these two application examples, a power consumption of around $500 \mathrm{~mW}$ is achieved mainly by reducing the operation speed of the visual co-processor.

The WiCa platform can be considered as the first approach to enable vision into WSN nodes which implements an architecture adapted to the nature of visual processing. During the processing of an image sequence, the greatest computational effort usually falls on the so-called early vision tasks. These tasks feature a very regular computational flow which must be applied 

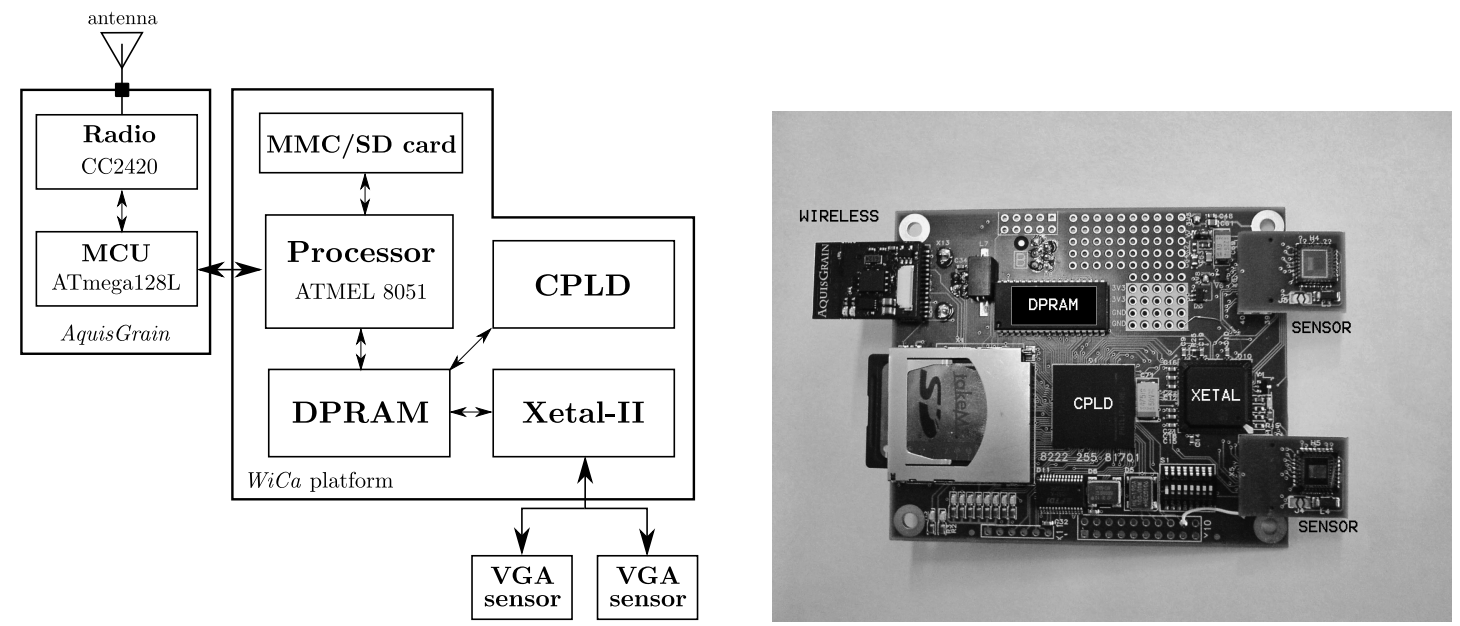

Figure 2.6: Interconnection scheme of $\mathrm{WiCa}$ system and snapshot of the platform (with permission of the authors).

independently to each element of a large data set. The integration of Xetal-II, a dedicated SIMDbased visual co-processor designed ad-hoc to deal only with early vision, improves the efficiency of the system by carrying out a parallel implementation of these tasks. Unlike pipeline schemes, this processor achieves full-parallel operation. In other words, the result of applying a certain instruction to a set of pixels is output at the same time for every pixel of the set, speeding up greatly the completion of the corresponding task.

\subsubsection{FireFly Mosaic [Rowe et al., 2007b]}

This platform consists of a FireFly [Rowe et al., 2006] wireless node coupled with a CMUcam3 [Rowe et al., 2007a] embedded vision processor. Its architecture is similar to that of Cyclops, described in Section 2.2.1. The computational resources for vision processing are again separated from those of wireless communication. CMUcam3 gathers the components concerning vision processing, namely: a CIF CMOS color image sensor [OV6620 (Omnivision)], a FIFO memory for buffering these images and a 32-bit ARM7 processor [LPC2106 (Philips)]. with 64KB of onchip RAM and 128KB of on-chip FLASH memory. On the other hand, FireFly node manages the wireless communication by means of an 8-bit MCU [ATmega1281 (ATMEL)] and an 802.15.4compliant radio transceiver [CC2420 (Texas Instr.)]. The interconnection CMUcam3-FireFly is carried out through 5 GPIO ports and a serial port. A sketch of the platform is shown in Fig. 2.7.

The main hardware difference between Cyclops and FireFly Mosaic is that the former needs a CPLD to complement the processor for image processing whereas the latter incorporates a more powerful processor which eliminates such a need. Another remarkable feature of FireFly Mosaic is the emphasis put on the problems related to transmitting information across a dense WSN. A time-synchronized link protocol for energy-constrained multi-hop wireless networks is 

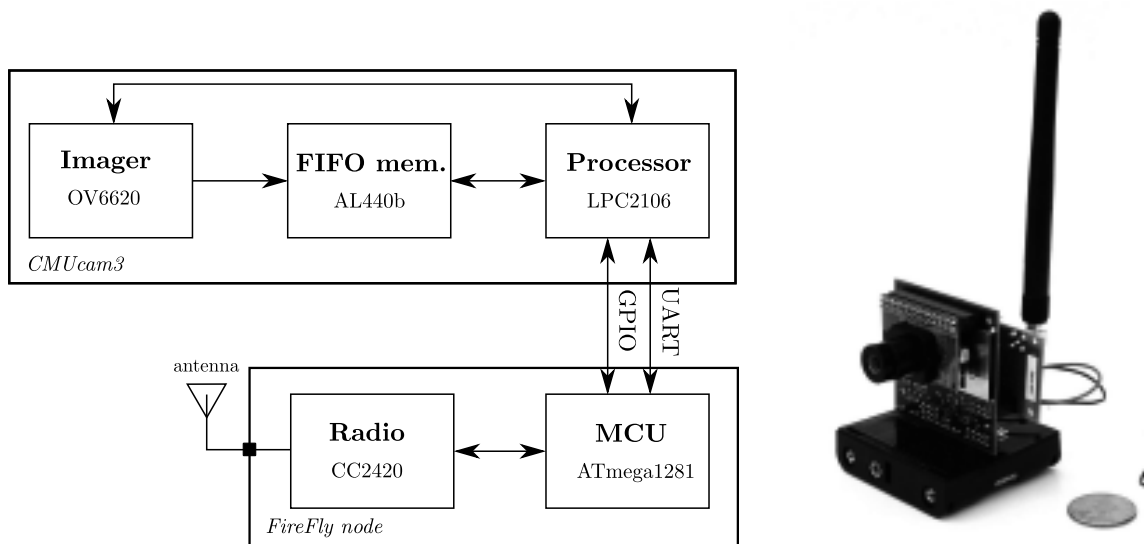

Figure 2.7: Block diagram of the FireFly Mosaic platform along with a snapshot of the whole system (with permission of the authors).

implemented. Regarding the power consumption, a maximum value of $572.3 \mathrm{~mW}$ is reported when all the components are active simultaneously, being the FIFO memory the most power consuming module with $171 \mathrm{~mW}$. The minimum value, with all the components sleeping, is $0.29 \mathrm{~mW}$.

Finally, an interesting application involving eight nodes is presented. These nodes are deployed throughout a house in order to extract relevant information about the activity occuring inside, for example a person falling. This information is obtained by a background subtraction algorithm and subsequent region clustering. The result is sent to a PC for further analysis. The frame rate at each node is $5.2 \mathrm{fps}$, operating at approximately $20 \%$ duty cycle. This means, according to the authors, that the motes can run for just over 5 days from four AA batteries.

\subsubsection{CITRIC [Chen et al., 2008]}

The general configuration of this platform is also very similar to Cyclops, as can be seen in Fig. 2.8. There is a dedicated processor [PXA270 (Marvell)] for visual processing which receives images from a 1280×1024px CMOS image sensor [OV9655 (Omnivision)]. This processor directly communicates with a commercial mote [TELOSB (MEMSIC)], in order to endow the system with wireless communication. An interesting feature of CITRIC is the integration of a microphone. The information sensed by this microphone is also analyzed by the vision processor, which therefore becomes a multimedia processor. It makes the field of application of this platform much wider.

Different figures of power consumption are reported assuming four AA batteries connected to the system. First, with CITRIC running Linux but with no active processes (idle), the power consumption ranges from $428 \mathrm{~mW}$ to $478 \mathrm{~mW}$, depending on the processor speed. Second, the same measurement is realized but now with TELOSB attached. No data is sent from CITRIC 

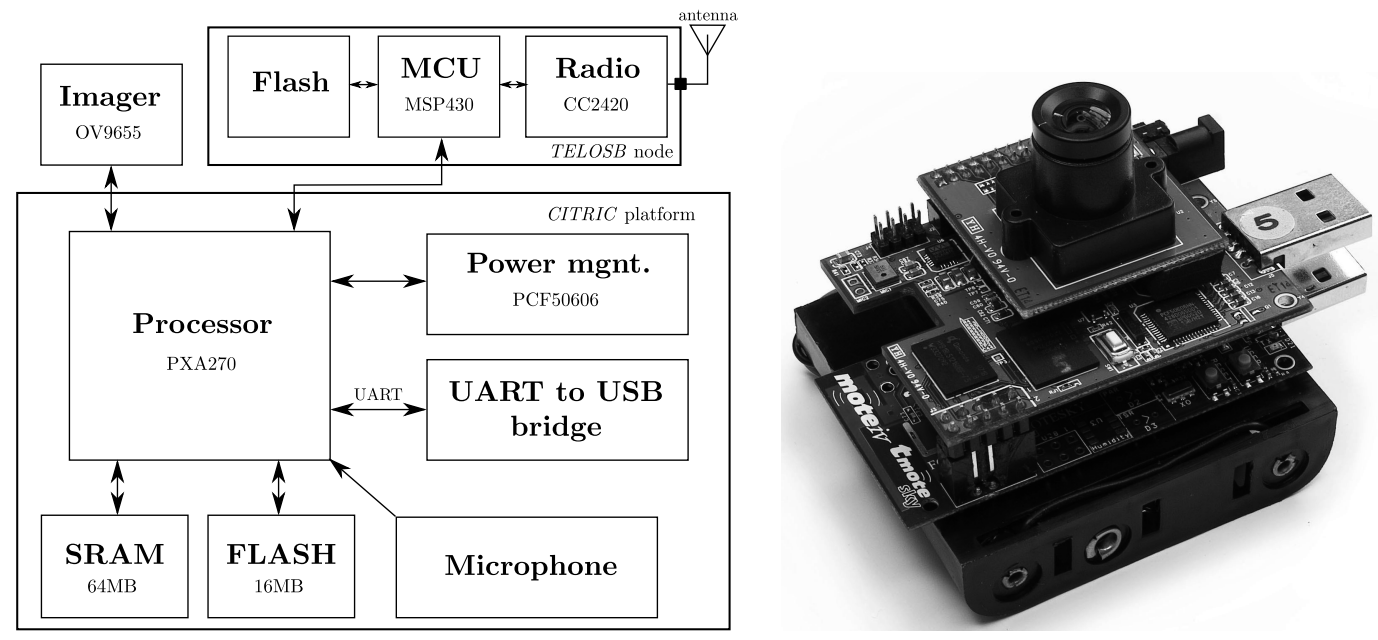

Figure 2.8: Block diagram of the CITRIC platform along with a snapshot of the whole system (with permission of the authors).

to TELOSB, although the latter is running an application that waits to receive any packets from the former and transmits them over the radio. The power consumption varies from $527 \mathrm{~mW}$ to $594 \mathrm{~mW}$. Finally, the power consumption running a typical background subtraction function is also measured. The test utilizes all the components of the system and processes images with a resolution of $512 \times 512 \mathrm{px}$. The image coordinates of the foreground are transmitted. For a processor speed of $520 \mathrm{MHz}$, the resulting power consumption is $970 \mathrm{~mW}$. In this case, the expected lifetime of the node would be 16 hours under continuous operation.

Three vision applications are presented: image compression, target tracking and camera localization. In all the cases, the processor speed is set to $520 \mathrm{MHz}$. Image compression consists of JPEG compression of $512 \times 512 \mathrm{px}$ test images. The computation time required is $70 \mathrm{~ms}$ per image, that is, a maximum frame rate of $14.3 \mathrm{fps}$ can be reached. The target tracking application is based on background subtraction via frame differencing. Each new frame is compared to a background image model, classifying the pixels with significant variation as part of the foreground. The foreground pixels are then processed for identification and tracking. Now, the execution time per frame is typically $0.2 \mathrm{~s}-0.4 \mathrm{~s}$ at a resolution of $320 \times 240 \mathrm{px}$ and $0.3 \mathrm{~s}-0.8 \mathrm{~s}$ at $640 \times 480 \mathrm{px}$. The frame rate is not fixed due to the variable execution time of the algorithm depending on the number of foreground pixels. Finally, camera localization is the process of finding the position of the cameras as well as the orientation of each camera's field of view. In this experiment, two vision-enabled CITRIC nodes are positioned $2.5 \mathrm{~m}$ apart focusing an open area with people walking. Each camera mote runs background subtraction on its current image and then sends the bounding box coordinates back to a base station for each detected foreground object. The center of each bounding box is used to build object tracks over time on the base station computer. By using these tracks, the position and orientation of the cameras can be estimated. 
As a summary, the CITRIC platform presents the incorporation of a microphone as a novelty, meaning the first attempt to integrate multimedia information — visual and audio processing - within the framework of WSNs. Another remarkable feature is the resolution of the CMOS image sensor, which is the highest one among the vision-enabled WSN nodes reported so far in the literature.

\subsubsection{EyeRIS ${ }^{\mathrm{TM}}$-based platform [Bakkali et al., 2010]}

This platform takes further than WiCa - see Section 2.2.4 - the adaptation of the architecture to the characteristics of visual processing. The vision capabilities of this WSN node are implemented by means of EyeRIS ${ }^{\mathrm{TM}}$ v1.2 [Rodríguez-Vázquez et al., 2008], a general-purpose programmable autonomous vision system. This system employs an architecture in which image processing is carried out by two hierarchical stages. In a first stage, low-level tasks are performed by analog circuitry at the very focal plane. Thus, concurrently with the photosensing, interconnected elementary analog building blocks process the image just captured in a massively parallel way. Local memories are incorporated to every block in order to avoid large consumption overheads due to repeated memory accesses. The result is an array of $176 \times 144$ sensing-processing cells, called $Q$-Eye, which realizes very efficiently different kind of early vision tasks over the image. The accuracy of these operations is moderate (6-7 bits), but enough for the subsequent digital processor, which constitutes the second processing stage. This 32-bit RISC digital processor [NIOS-II (Altera)] performs higher abstraction tasks by making use of the pre-processed images coming from the sensor-processor. Complex algorithms with long and irregular computational flows leading to decision making are typical for this second stage. As a whole, the processing architecture implemented by EyeRIS ${ }^{\mathrm{TM}}$ emulates the organization of the retina [Masland, 2001]. In natural vision systems, the visual information is not only acquired but also preprocessed in the focal-plane device, the retina, before being sent to the visual cortex through the optic nerve for further understanding. Interestingly, this pre-processing is performed in the analog domain by means of retinal cells organized in layers and interacting locally [Roska \& Werblin, 2001].

Regarding the wireless communication, a commercial node [IRIS (MEMSIC)] is utilized. This platform includes an MCU [ATmega1281 (ATMEL)] and an IEEE 802.15.4-compliant RF transceiver [CC2420 (Texas Instr.)]. The EyeRIS ${ }^{\mathrm{TM}}$-to-IRIS interconnection is carried out through either UART or GPIO pins available from both systems. A sketch of the resulting WSN node along with a snapshot, reproduced here with permission of the authors, are depicted in Fig. 2.9.

Three examples of possible applications are presented. The first one consists of broadcasting a motion-triggered alarm signal. A basic algorithm detects changes in an image sequence. When a change is detected, an alarm signal is transmitted to the IRIS through a GPIO pin in order to 

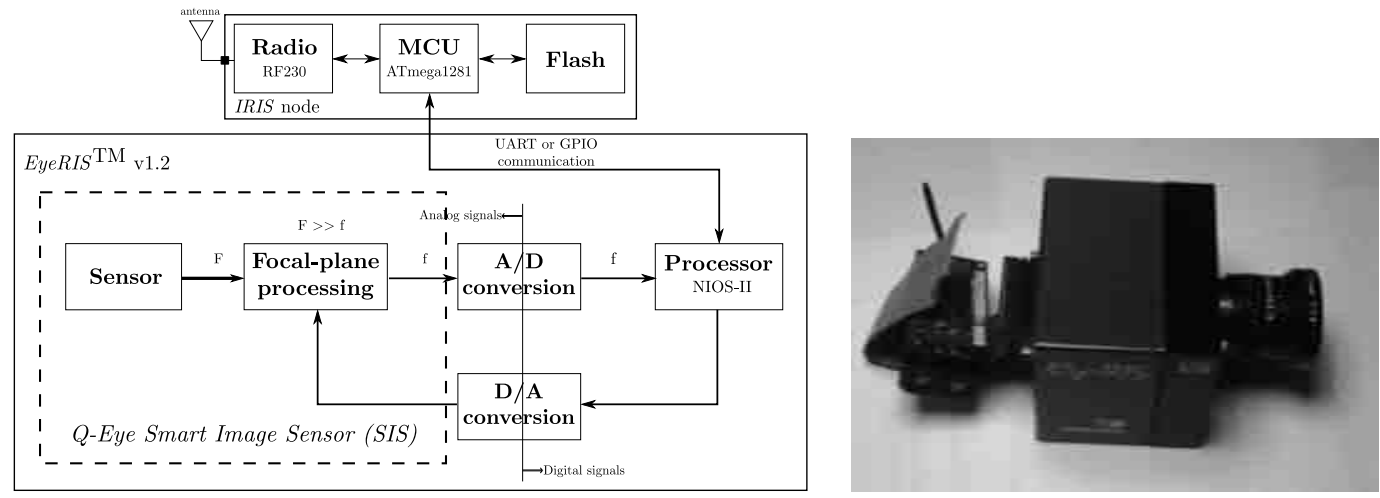

Figure 2.9: Block diagram of EyeRIS ${ }^{\mathrm{TM}}$-based WSN node and snapshot of the platform (with permission of the authors).

be broadcasted. The second example shows some of the possibilities of the EyeRIS ${ }^{\mathrm{TM}}$ system. The coordinates of a targeted ROI are extracted and delivered through UART to the IRIS, which broadcasts them. Finally, the result of an in-situ image flow analysis is also broadcasted. Specifically, a code indicating the sense of the major motion component within the sequence is delivered to the IRIS to be transmitted.

Summarizing, this vision-enabled WSN node achieves a very high degree of energy efficiency by implementing an architecture fully adapted to the features of visual processing. This adaptation does not only consist, like in Xetal-II processor described in Section 2.2.4, of taking advantage of the potential parallel processing of early vision tasks, which are now performed at the focal plane. In addition, EyeRIS ${ }^{\mathrm{TM}}$ system benefits from the moderate accuracy [Poynton, 2007] required for such tasks. Thus, by making use of analog building blocks, not too accurate but very fast and energy-efficient, this platform exhibits a computational power of 250GOPS with a power consumption of $4 \mathrm{~mW} /$ GOPS. This figure improves the $5.6 \mathrm{~mW} /$ GOPS of Xetal-II. Nevertheless, despite the computational power and energy efficiency of EyeRIS ${ }^{\mathrm{TM}}$, its power consumption, $1.5 \mathrm{~W}$, is still too high to achieve long node lifetime in real deployments of WSNs.

\subsection{Comparative analysis}

Some of the features of the vision-enabled WSN nodes summarized in the previous section have been gathered in Table 2.1. Its main objective is to have access to key aspects of the different platforms with a quick look. It also allows for a fast comparison of interesting data such as camera resolution or type of processor. Nevertheless, it is important to remark at this point that a fair comparison concerning the most important issue in a vision-enabled WSN node, that is, power consumption, is not possible. It is due to the heterogeneity of the application framework over which the different systems have been tested. As an example, consider the last two columns in Table 2.1. We have put together here a very general description of one of the applications 
reported for every platform along with the power consumption measured for that application, the resolution of the images processed and the frame rate reached. It can be seen that there is not a single match, even though we have chosen the most similar set of applications. This absence of a common application testbench for vision-enabled WSN nodes prevents us from establishing an adequate comparison of the energy efficiency of the different approaches. Such a testbench should define a prescribed vision algorithm to be run under prescribed light conditions. It should also define the size of the images to be processed as well as the frame rate. Under these circumstances, each platform could be adjusted to fairly compete with the rest for delivering the targeted result with minimum power consumption. As an attempt to provide a FOM for a comparison of this set of applications, the last column also includes the energy per pixel required by each platform to complete the corresponding algorithm. But we must insist about the weakness of such a comparison.

Another noteworthy detail clearly highlighted by Table 2.1 is that applications tend to process images with very low resolution, specially when compared to the maximum possible resolution of the associated imager. By working with low resolution images, the power consumption can be significantly reduced for a prescribed frame rate. Or, from the opposite point of view, for a prescribed power consumption, a higher frame rate can be obtained. The fact is that currently the integration of a high-resolution imager could be counterproductive because of the extra power consumption associated to the capture of the entire pixel array.

Finally, notice that all platforms but Cyclops and WiCa make use of 32-bit processors. Apparently, 8-bit processors are lighter and therefore consume less power. However, 32-bit processors are better suited for image processing than their 8-bit counterparts. In [Downes et al., 2006], an interesting experiment is carried out. The time needed to perform operations such as 2-D convolution on an 8-bit processor clocked at $4 \mathrm{MHz}$ is 16 times higher than with a 32-bit ARM7 device clocked at $48 \mathrm{MHz}$, while the power consumption of the 32-bit processor is only six times higher. Hence, an 8-bit processor turns out to be slower and less energy-efficient. In fact, Cyclops needs a CPLD to be able to reach acceptable frame rates for the applications considered. On the contrary, WiCa can afford a less powerful processor due to its visual co-processor performing the most computationally heavy tasks.

\subsection{Guidelines for a power-aware vision-enabled wireless node}

WSNs can be considered as an essential building block for the paradigm of ubiquitous computing. Its field of application is currently very extensive, but it can be significantly boosted by the incorporation of multimedia sensing within the catalog of sensing capabilities of the nodes. 


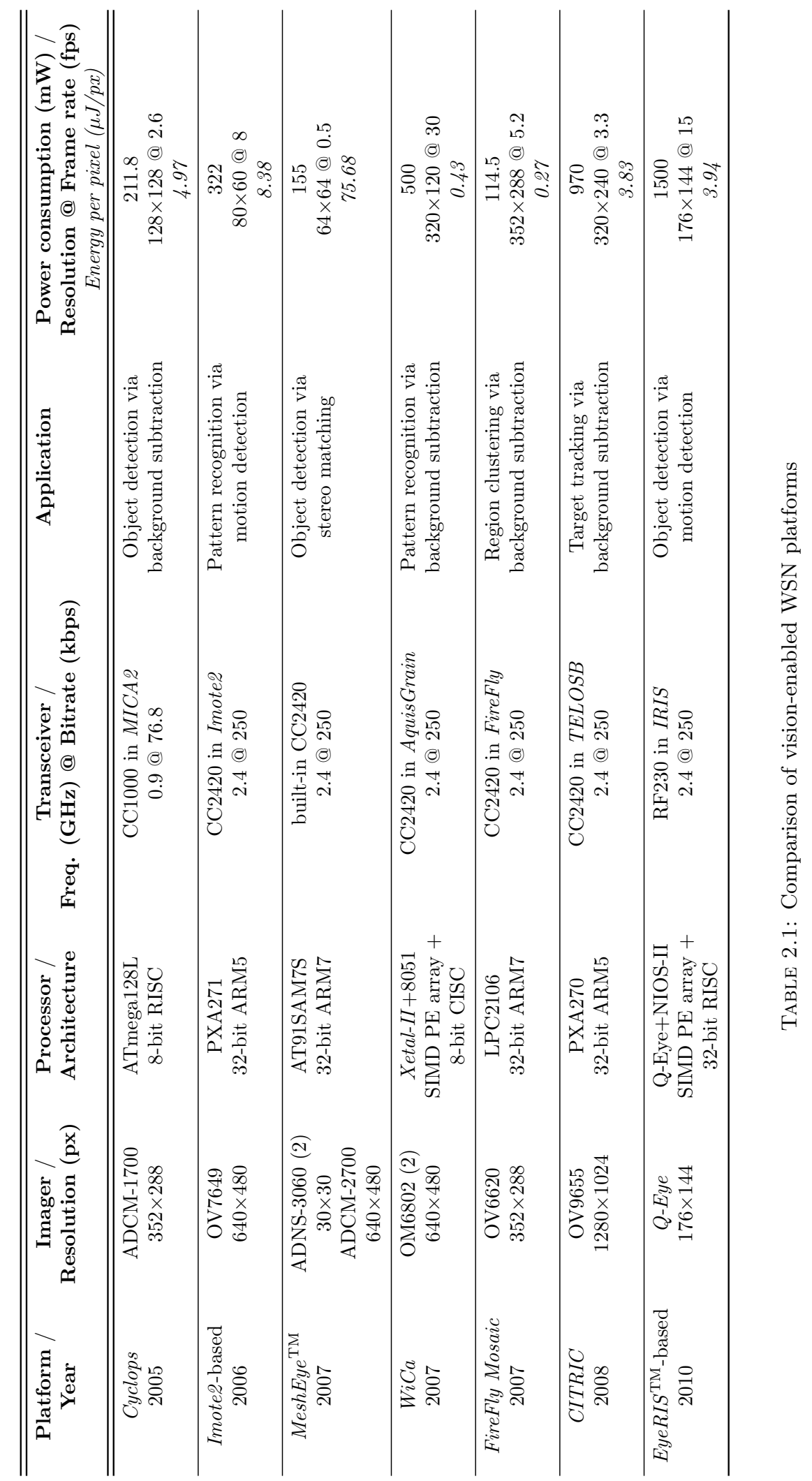




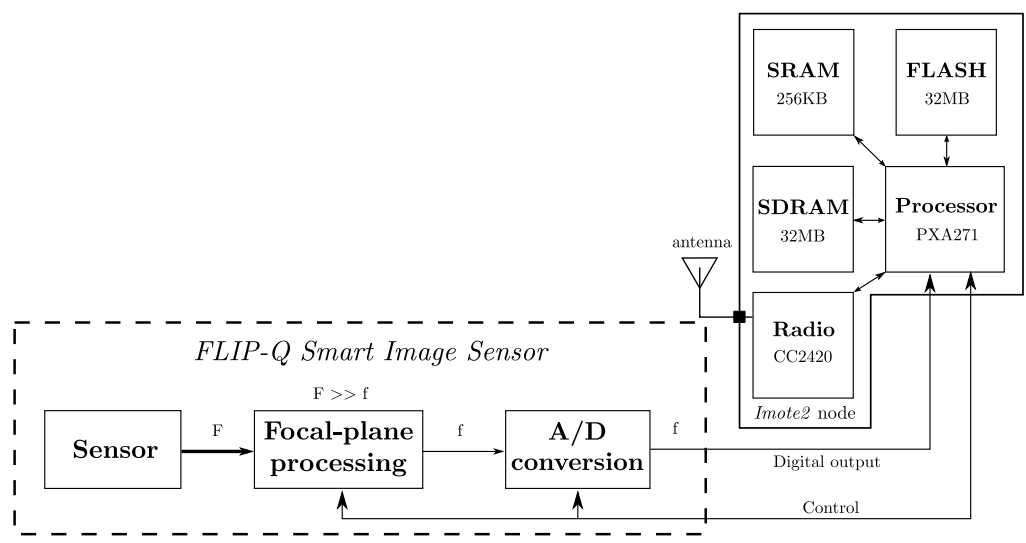

Figure 2.10: Basic sketch of the vision-enabled WSN node proposed.

Specifically, the integration of vision has become a burning issue within the research community. In this chapter, a survey of different approaches reported to carry out this integration has been realized. Most of them implement conventional processing architectures. Images are captured by a sensor and immediately converted into the digital domain. The resulting serialized digital flow is usually handled by a single processor, which performs all the processing required by the corresponding algorithm. However, the special characteristics of this digital flow - massive and multidimensional - as well as of the low-level tasks commonly applied during early stages of image processing - regular and potentially parallel — have led to more efficient alternative approaches. The WiCa platform represents the first attempt to address differently such special characteristics by making use of a high-performance parallel digital co-processor. This co-processor, dedicated only to vision, takes advantage of the regularity and parallelism of early image processing to reduce the number of memory accesses, clock speed and instruction decoding. The EyeRIS ${ }^{\mathrm{TM}}$-based platform goes further in the adaptation of the architecture to image processing. In this case, low-level tasks are carried out in a massively parallel way at the very focal plane. The imager does not already consist only of photosensing devices and some additional readout circuitry. Now, it becomes a really smart camera where distributed PEs influence the image capture in order to deliver a simplified representation of the scene. These PEs, which work very efficiently in analog mode, reach equivalent resolutions of about 6-7 bits. It is enough for the subsequent digital processor, which now receives pre-processed images over which apply medium- and high-level processing. As a whole, the EyeRIS ${ }^{\mathrm{TM}_{-}}$-based platform exhibits a better performance than WiCa. The main drawback of the WiCa and EyeRIS ${ }^{\mathrm{TM}}$-based platforms is their high power consumption. Despite their computational power and energy efficiency, their design is not specifically oriented to WSNs. On the contrary, their scope of application is very wide thanks to the great deal of functionalities and prominent features available. But these functionalities and prominent features also mean that the power consumption of the system is higher. Indeed, too high for the real requirements of vision-enabled WSN applications. 
All in all, our proposal is based on a processing architecture fully adapted to the special characteristics of WSNs. Thus, we address the VLSI implementation of a prototype vision chip delivering a set of focal-plane processing primitives which has been selected with the requirements of WSN nodes in mind. Minimum power consumption and image simplification represent the driving forces for the design of the chip. As a result, the prototype outputs a digital data flow containing reduced representations of the scene obtained at ultra low energy cost. This energy efficiency together with the programmability of the focal-plane processing have enabled the direct integration of the chip with Imote2, the commercial node already mentioned at different points of this chapter. It is not necessary now the separation of the computational resources assigned for image processing from those assigned for communication. Under normal circumstances, the PXA271 processor of Imote2 will be able to deal with both image processing and asynchronous requirements of the network as its computational load has been greatly alleviated by the WSN-oriented smart vision chip. A basic sketch of the resulting system is depicted in Fig. 2.10. As a first step for its comprehensive description, we next analyze and justify the set of focal-plane processing primitives chosen to be implemented in the prototype while developing the mathematical framework which supports them. 



\section{Chapter 3}

\section{Processing primitives for image simplification}

We have already seen in Chapter 2 that the simplification of the scene is a key issue when it comes to save energy in vision-enabled WSN nodes. It permits to reduce the clock speed of the digital processor as well as the number of memory accesses, two important parameters concerning the power consumption of the nodes. Obviously, the energy cost of obtaining a simplified version of the scene and processing it must be lower than the energy cost of directly processing the raw representation of the scene. Therefore, it does not suffice to define image processing primitives capable of achieving such simplification. Their hardware implementation also plays a crucial role in order to reach a really high degree of energy efficiency.

This chapter deals only with the mathematical definition of processing primitives whose application reduces, to a user-defined extent, the amount of data representing an image. As a matter of course, the choice of these primitives was jointly realized with the study of their feasibility in terms of a very power-efficient VLSI implementation. But let us now forget about the physical implementation, which will be addressed thoroughly in the following chapters, and focus only on the theory behind these primitives.

\subsection{Gaussian filtering}

Gaussian filtering is a basic task for early vision. It is used for reducing the noise associated with the image capture without affecting subsequent processing stages. In fact, image enhancement through the Difference of Gaussians (DoG) is preferred over other image enhancement methods as it preserves the details of interest within the scene while filtering sharp random noise [Poggio 
et al., 1988]. The width of the Gaussian filters involved will depend on the nature of this noise. Another interesting application framework for Gaussian filtering is scale-space representation [Lindeberg, 1994]. The scale space of a scene is obtained by applying successive Gaussian filters with increasing widths over its original representation. Scale is indeed a key point when it comes to efficiently process images. Its adequate selection simplifies the analysis of certain features in a scene by removing information at other scales [Mutch \& Lowe, 2008]. If several scales are to be analyzed, pyramidal representations can be built in order to only store the necessary information for each representation according to the spatial frequencies of interest [Jahne, 1999].

From the processing features just described, it can be seen that the utility of Gaussian filtering reaches its maximum level when the width, or smoothing degree, is under a fine control of the user. Conventional digital processors support this possibility by means of, for example, userdefined convolution kernels or FFT-based algorithms. However, their implementation is not very efficient, taking into account the necessary serialization of the raw image data along with the repeated accesses to memory to operate over each and every pixel and its neighborhood.

\subsubsection{Continuous-space linear diffusion}

An alternative to obtain Gaussian filtering is a diffusion process. The concept of diffusion is widely applied in physics. It explains the equalization process undergone by an initially uneven concentration of a certain magnitude. A typical example is heat diffusion. Mathematically, a diffusion process can be defined by considering a function $V(\mathbf{x}, t)$ defined over a continuous space, in this case a plane, for every time instant. At each point $\mathbf{x}=\left(x_{1}, x_{2}\right)$, the linear diffusion of the function $V($.$) is described by the following well-known PDE [Jahne, 1999]:$

$$
\frac{\partial V}{\partial t}=\nabla \cdot(D \nabla V)
$$

where $D$ is referred to as the diffusion coefficient. If $D$ does not depend on the position:

$$
\frac{\partial V}{\partial t}=D \nabla^{2} V
$$

and realizing the spatial Fourier transform of this equation, we obtain:

$$
\frac{\partial \hat{V}(\mathbf{k})}{\partial t}=-4 \pi^{2} D|\mathbf{k}|^{2} \hat{V}(\mathbf{k})
$$

where $\mathbf{k}$ represents the wave number vector in the continuous Fourier domain. Finally, by solving this equation we have: 


$$
\hat{V}(\mathbf{k}, t)=\hat{V}(\mathbf{k}, 0) e^{-4 \pi^{2} D t|\mathbf{k}|^{2}}
$$

where $\hat{V}(\mathbf{k}, t)$ is the spatial Fourier transform of the function $V($.$) at time instant t$ and $\hat{V}(\mathbf{k}, 0)$ is the spatial Fourier transform of the function $V($.$) at time t=0$, that is, just before starting the diffusion. Eq. (3.4) can be written as a transfer function:

$$
\hat{G}(\mathbf{k}, t)=\frac{\hat{V}(\mathbf{k}, t)}{\hat{V}(\mathbf{k}, 0)}=e^{-4 \pi^{2} D t|\mathbf{k}|^{2}}
$$

which, by defining $\sigma=\sqrt{2 D t}$, is transformed into:

$$
\hat{G}(\mathbf{k}, \sigma)=e^{-2 \pi^{2} \sigma^{2}|\mathbf{k}|^{2}}
$$

This transfer function corresponds to the Fourier transform of a spatial Gaussian filter of the form:

$$
G(\mathbf{x}, \sigma)=\frac{1}{2 \pi \sigma^{2}} e^{-\frac{|\mathbf{x}|^{2}}{2 \sigma^{2}}}
$$

and therefore the diffusion process is equivalent to the convolution expressed by the following equation:

$$
V(\mathbf{x}, t)=\frac{1}{2 \pi \sigma^{2}} e^{-\frac{|\mathbf{x}|^{2}}{2 \sigma^{2}}} * V(\mathbf{x}, 0)
$$

We can see that a diffusion process intrinsically entails a spatial Gaussian filtering which takes place along time. The width of the filter is determined by the time the diffusion is permitted to evolve: the longer the diffusion time, $t$, the larger the width of the corresponding filter, $\sigma$. This means that, ideally, any width is possible provided that a sufficiently fine temporal control is available. From the point of view of the Fourier domain, we can define the diffusion as an isotropic lowpass filter whose bandwidth is controlled by $t$. The longer $t$, the narrower the bandwidth of the filter around the dc component (Fig. 3.1). Eventually, for $t \rightarrow \infty$, all the spatial frequencies but the dc component are removed. Furthermore, this dc component is completely unaffected by the diffusion, that is, $\hat{G}(\mathbf{0}, t)=1 \forall t$.

\subsubsection{Discrete-space linear diffusion}

In order to apply the concept of diffusion to image processing, it is necessary to discretize the space within which it occurs. Let us map Eq. (3.2) into a bidimensional grid. Only the values at 

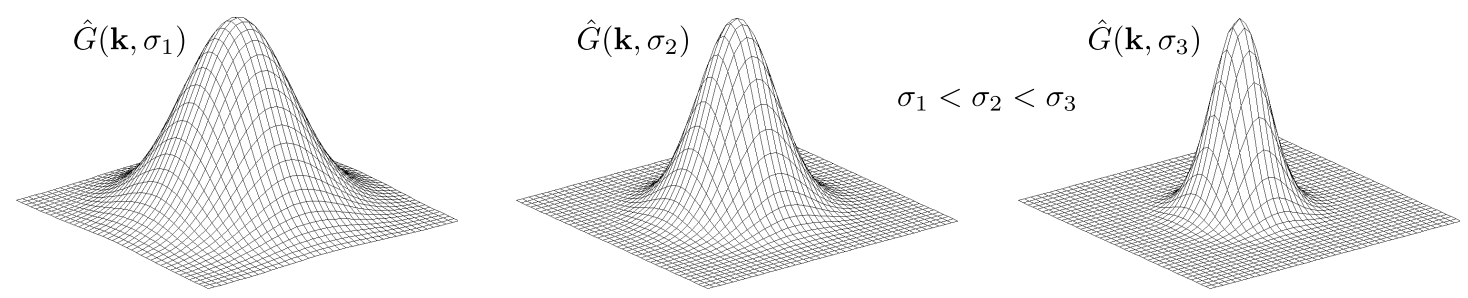

Figure 3.1: Diffusion-based spatial Gaussian filters with increasing $\sigma$ represented in the Fourier domain.

specific points, which will be indexed by $i$ and $j$, are considered of interest. Using the following approximation of the Laplacian operator:

$$
\begin{array}{r}
\nabla^{2} V \simeq \frac{V(x+\Delta x, y)-2 V(x, y)+V(x-\Delta x, y)}{(\Delta x)^{2}}+ \\
+\frac{V(x, y+\Delta y)-2 V(x, y)+V(x, y-\Delta y)}{(\Delta y)^{2}}
\end{array}
$$

and taking into account that, due to the discretization, the minimum possible value of $\Delta x$ and $\Delta y$ is one, we have at each node of the grid:

$$
\frac{d V_{i j}}{d t}=D\left(V_{i+1, j}+V_{i-1, j}+V_{i, j+1}+V_{i, j-1}-4 V_{i j}\right)
$$

By applying the DFT to this equation, the following expression is obtained:

$$
\frac{1}{D} \frac{d \hat{V}_{u v}}{d t}=-4 \hat{V}_{u v}+e^{\frac{2 \pi i u}{M}} \hat{V}_{u v}+e^{\frac{-2 \pi i u}{M}} \hat{V}_{u v}+e^{\frac{2 \pi i v}{N}} \hat{V}_{u v}+e^{\frac{-2 \pi i v}{N}} \hat{V}_{u v}
$$

where we have considered a grid whose size is $M \times N$ points, in our case $M \times N$ pixels. Eq. (3.11) can be rewritten as:

$$
\frac{1}{D} \frac{d \hat{V}_{u v}}{d t}=-4\left[\sin ^{2}\left(\frac{\pi u}{M}\right)+\sin ^{2}\left(\frac{\pi v}{N}\right)\right] \hat{V}_{u v}
$$

and solving now in the time domain we obtain the following transfer function:

$$
\hat{G}_{u v}(t)=\frac{\hat{V}_{u v}(t)}{\hat{V}_{u v}(0)}=e^{-4 D t\left[\sin ^{2}\left(\frac{\pi u}{M}\right)+\sin ^{2}\left(\frac{\pi v}{N}\right)\right]}
$$

where $\hat{V}_{u v}(0)$ represents the DFT of the grid defined by the initial values of the pixels and $\hat{V}_{u v}(t)$ is the DFT of the grid defined by the values of the pixels after a certain time interval $t$ since the diffusion started to evolve. Therefore, Eq. (3.13) describes the filtering process undergone by the 


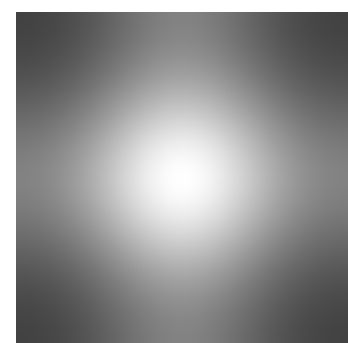

$\hat{G}_{u v}\left(t_{1}\right)$

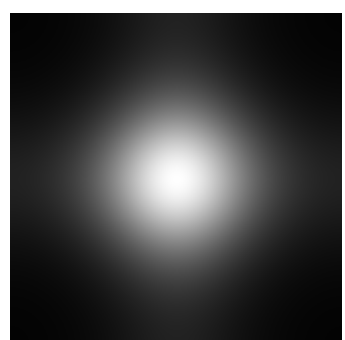

$\hat{G}_{u v}\left(t_{2}\right)$

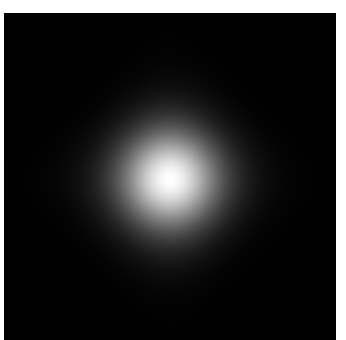

$\hat{G}_{u v}\left(t_{3}\right)$

$t_{1}<t_{2}<t_{3}$

FiguRE 3.2: Diffusion-based spatially-discretized Gaussian filters with increasing $t$ represented over the discrete Fourier plane (overhead view).

pixels as diffusion progresses. It is an approximation of the isotropic diffusion process defined by Eq. (3.5). In fact, for small enough values of $(\pi u / M)$ and $(\pi v / N)$, we have:

$$
\hat{G}_{u v}(t) \simeq e^{-4 \pi^{2} D t|\mathbf{k}|^{2}}
$$

where $|\mathbf{k}|^{2}=(u / M)^{2}+(v / N)^{2}$. Only under these conditions, the right term in Eq. (3.14) does coincide exactly with its counterpart for continuous space in Eq. (3.5). In terms of image processing, this means that the filtering performed by a spatially-discretized linear diffusion is not perfectly isotropic. The reason is precisely the process of discretization itself, which constrains the filtering of the highest spatial frequencies to a reduced number of spatial directions. The lower the frequencies considered, the more the possible spatial directions involved in the filtering and therefore the more its isotropic shape. In order to better understand this consequence of the discretization, we have represented in Fig. 3.2 the transfer function defined by Eq. (3.13) with increasing values of $t$ for a square grid. An overhead view of the discrete Fourier plane is now provided. The center of the plane corresponds to the indexes $(u, v)=(0,0)$, that is, the dc component. Similarly to the continuous-space case, this component is completely unaffected by the diffusion, that is, $\hat{G}_{00}(t)=1 \forall t$. Note that for short values of $t$, the highest frequencies are not filtered evenly. However, the longer the interval $t$, the higher the frequencies removed and therefore the more isotropic the filtering is.

Aside this inherent, otherwise unavoidable, effect of the discretization, we can consider discretespace linear diffusion as a generator of ideally any possible 2-D Gaussian filter. The width of the filter keeps being determined by the time the diffusion is allowed to evolve through the expression $\sigma=\sqrt{2 D t}$. Consequently, the finer the temporal control of the diffusion, the larger the family of filters attainable. Another important property of the diffusion process is the invariance of the dc component. In practical terms, this implies that, provided a long enough time interval of 


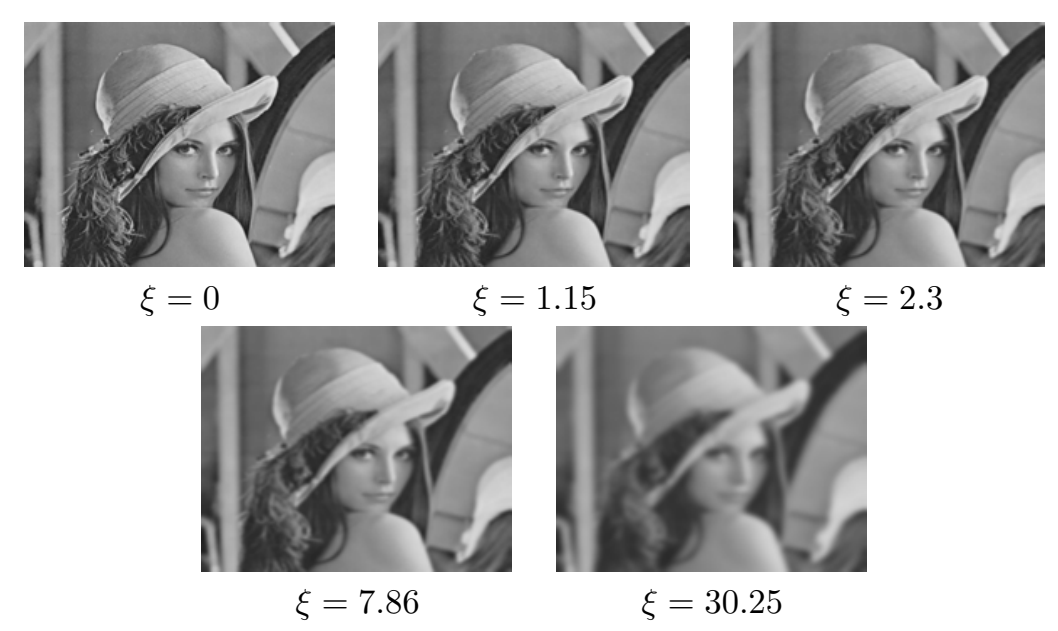

FiguRE 3.3: Scale-space representation (5 scales) of the $512 \times 512$-px Lena image.

diffusion, all the pixels diffused will reach the same value, their mean. We will see shortly the potentiality of such a simple property when applied to user-defined independent blocks of pixels.

\subsubsection{Scale-space representation}

We observed at the beginning of this section the importance of scale when it comes to efficiently process images. Interestingly, the solution of Eq. (3.10) is formally the scale-space representation of 2-D discrete signals [Lindeberg, 1991]. Therefore, time-controlled linear diffusion can deliver the scale-space representation of a scene. In fact, the scale parameter associated with this representation is defined as $\xi=\sigma^{2}=2 D t$. As an example, consider the scale-space representation (5 scales) of the $512 \times 512$-px Lena image depicted in Fig. 3.3 .

\subsubsection{Gaussian pyramids}

Scale-space representations successively become more redundant as the scale parameter increases. A progressive filtering is performed over the scene, starting from the highest spatial frequencies and continuing until eventually filtering all the frequencies other than the dc component. However, in this process, the resolution of the images does not change and the oversampling of the remaining frequency content constantly increases along the scale space. Pyramid representations solve this problem by subsampling the scale-space representation according to the filtering realized. For example, we have depicted in Fig. 3.4 the Gaussian pyramid associated with the scale space of Fig. 3.3. The scales of this scale space were chosen in such a way that the corresponding pyramid could be adequately built. Thus, for $\xi=1.15$, the components of the DFT at the highest vertical and horizontal frequencies, denoted respectively as $(u, v)=(M / 2,0)$ and $(u, v)=(0, N / 2)$, suffer a decrease on their magnitude by a factor of 0.1 - we have applied 


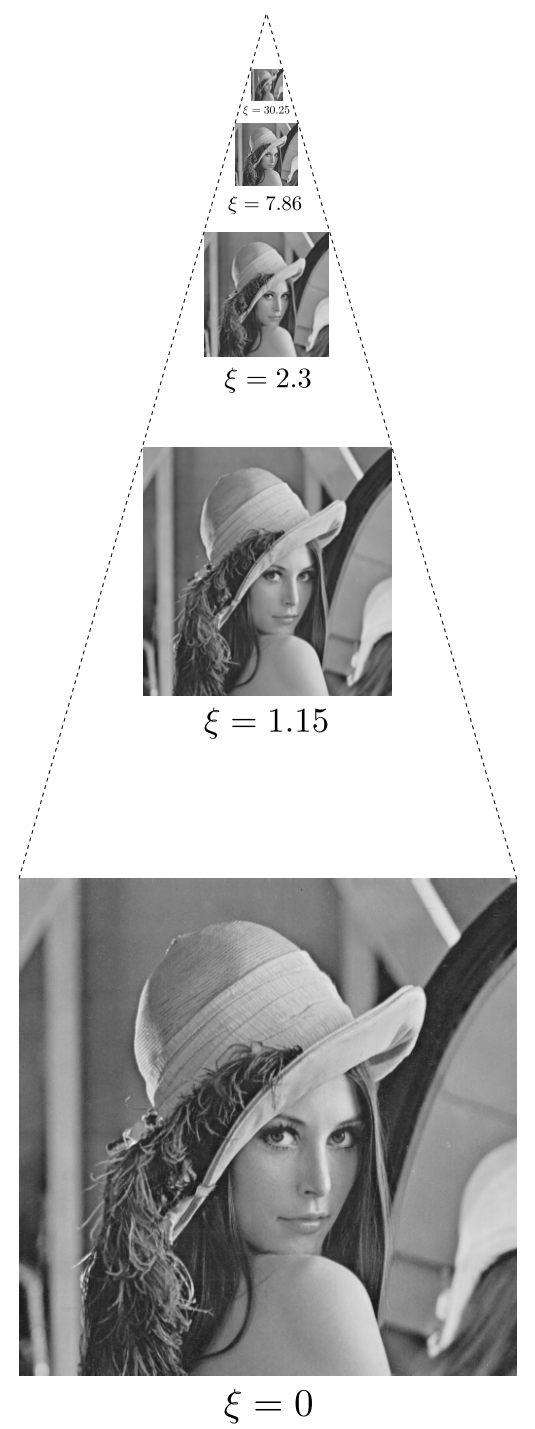

FIGURE 3.4: Gaussian pyramid associated with the scale-space representation of Fig. 3.3.

Eq. (3.13). Consequently, their energy is reduced to just a $1 \%$ of its value at $\xi=0$, so they have lost $99 \%$ of their energy. We therefore assume that a subsampling factor equal to 2 can be applied over the vertical and horizontal dimensions of the image without significant loss of information. For $\xi=2.3$, both components $(u, v)=(M / 2,0)$ and $(u, v)=(0, N / 2)$ have been even more attenuated and, additionally, $(u, v)=(M / 4,0)$ and $(u, v)=(0, N / 4)$ have also lost around the $99 \%$ of their energy. In this case, a subsampling factor equal to 4 can be applied without losing relevant information. Similarly, $\xi=7.86$ and $\xi=30.25$ allow a subsampling factor equal to 8 and 16 , respectively. 


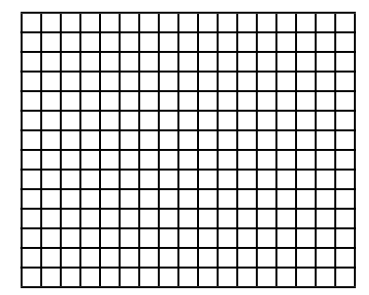

(a)

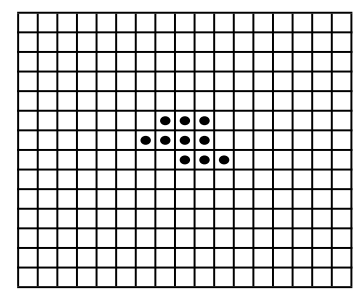

(b)

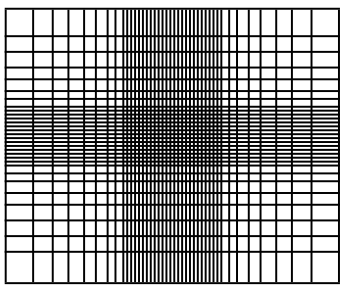

(c)

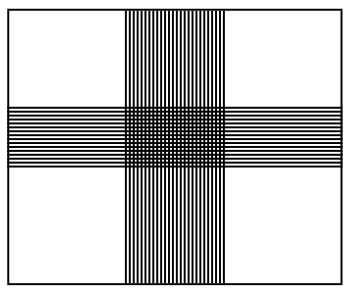

(d)

Figure 3.5: Example of dynamic image plane division: (a) Regular division; (b) Blocks of interest detected; (c) Gradual image plane division with maximum granularity in the ROI; (d) Abrupt image plane division with maximum granularity in the ROI and minimum for the rest.

\subsection{Reconfigurable block-wise image plane division}

A simple way of reducing the representation of a scene is to pay careful attention only to those regions that at any moment and for any reason are of interest. The rest of the scene keeps being surveyed, but with a coarser detail. To this end, a very useful tool consists of a dynamic content-based image plane division. Consider for example Fig. 3.5. We start with a regular division grid (Fig. 3.5(a)) where every block is independently processed. At a certain time instant, a higher cognitive level functionality determines that some blocks, marked with a black dot in Fig. 3.5(b), contain information of interest. According to their location, the image plane is reconfigured in such a way that the size of the blocks gradually shrinks (Fig. 3.5(c)). The blocks marked are divided into much smaller blocks for fine-grain processing. As we get farther from the region of interest (ROI), the blocks become progressively bigger. As a result, a content-aware adaptive representation of the scene is obtained. The information accounting for the ROI is very detailed while increasingly simplified data is provided for the rest. Another option would be to keep maximum granularity in the ROI and minimum for the rest (Fig. 3.5(d)). Note that we are assuming that the division is established row-wise and column-wise. That is to say, the boundaries of the blocks are determined by isolating neighboring rows and columns across the entire image plane. This restriction makes the physical implementation of a reconfigurable image plane simpler and easier to program, as will be seen in Chapter 5.

\subsection{Multiresolution image representation}

The possibilities of simplifying an image are boosted by combining linear diffusion and reconfigurable image plane division. Consider a diffusion process taking place independently within each block defined by a regular division grid like that of Fig. 3.5(a). If we let diffusion run for a long enough time interval, all the pixels comprising every block would end up reaching the same value, their mean. That is: 


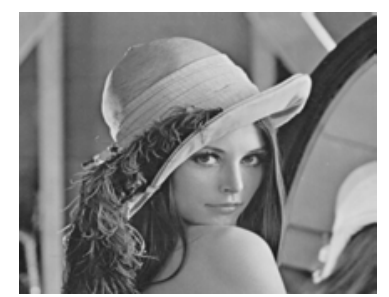

(a)

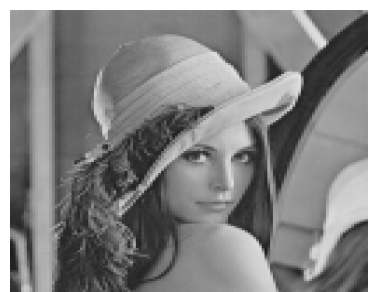

(b)

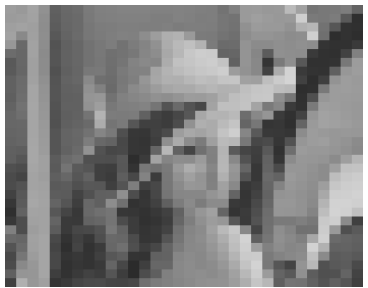

(c)

Figure 3.6: Examples of regular multiresolution representation: (a) Original Lena image; (b) Regular 4×4-px division; (c) Regular 16×16-px division.

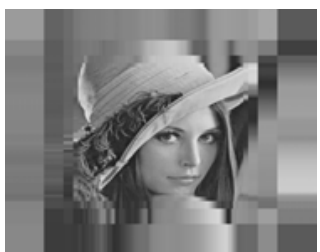

(a)

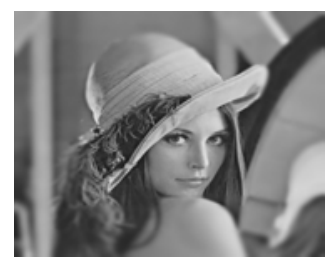

(b)

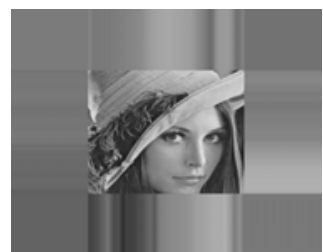

(c)

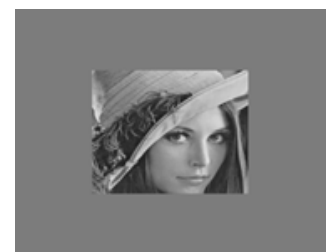

(d)

Figure 3.7: Examples of foveation: (a) Application of a gradual division scheme like that of Fig. 3.5(c); (b) Corresponding ideal gradual foveation; (c) Application of an abrupt division grid like that of Fig. 3.5(d); (d) Corresponding ideal abrupt foveation.

$$
p_{i j_{k l}}^{\prime}=\frac{1}{W H} \sum_{i=1}^{W} \sum_{j=1}^{H} p_{i j_{k l}} \quad \forall(i, j) \in(k, l)
$$

where $p_{i j_{k l}}^{\prime}$ represents the resulting value of the pixel $(i, j)$ belonging to the block $(k, l)$ after finishing diffusion, $W \times H$ is the size, in pixels, of the block under consideration, and $p_{i j_{k l}}$ is the value of the pixel $(i, j)$ belonging to the block $(k, l)$ before diffusion started. As an example, we have applied over a $512 \times 512$-px Lena image (Fig. 3.6(a)) a regular division grid of $4 \times 4 \mathrm{px}$ (Fig. 3.6(b)) and $16 \times 16$ px (Fig. 3.6(c)). By subsampling one pixel out of every block, this operation leads to multiresolution image representation, which can be seen as a simplified version of a Gaussian pyramid. Now, unlike in Gaussian pyramids, the original full-resolution image is not adequately filtered for the subsequent subsampling. On the other hand, multiresolution representation is simpler to obtain than Gaussian pyramids as it is based on averaging. No fine temporal control of diffusion is necessary.

This same operation with division schemes like those of Fig. 3.5(c) and Fig. 3.5(d) generates very simplified versions of a gradual and an abrupt foveation, respectively. In Fig. 3.7(a), we make use of a gradual division grid which is applied again to the $512 \times 512$-px Lena image. The corresponding ideal gradual foveation, obtained by selective subsampling along the scale space, is depicted in Fig. 3.7(b). Similarly, Fig. 3.7(c) represents the result of applying an abrupt block-wise division whereas Fig. 3.7(d) corresponds to its ideal counterpart. 


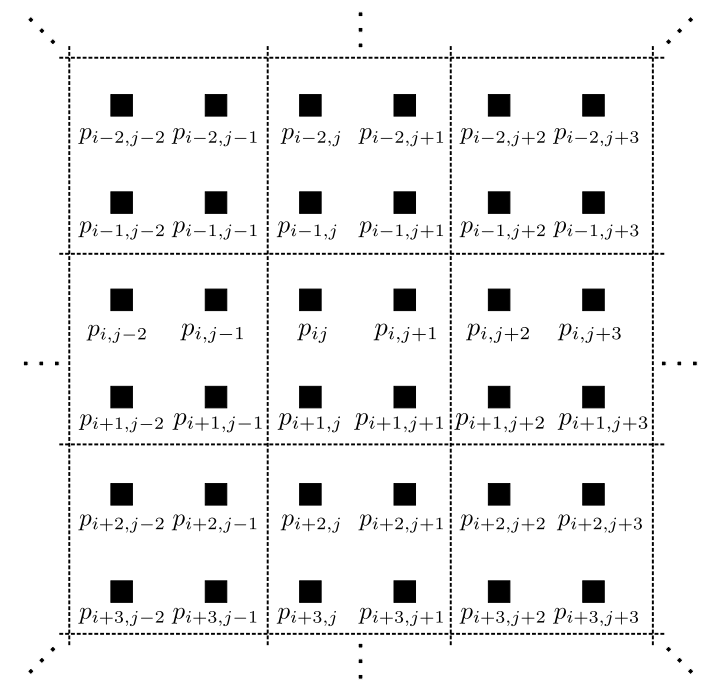

(a)

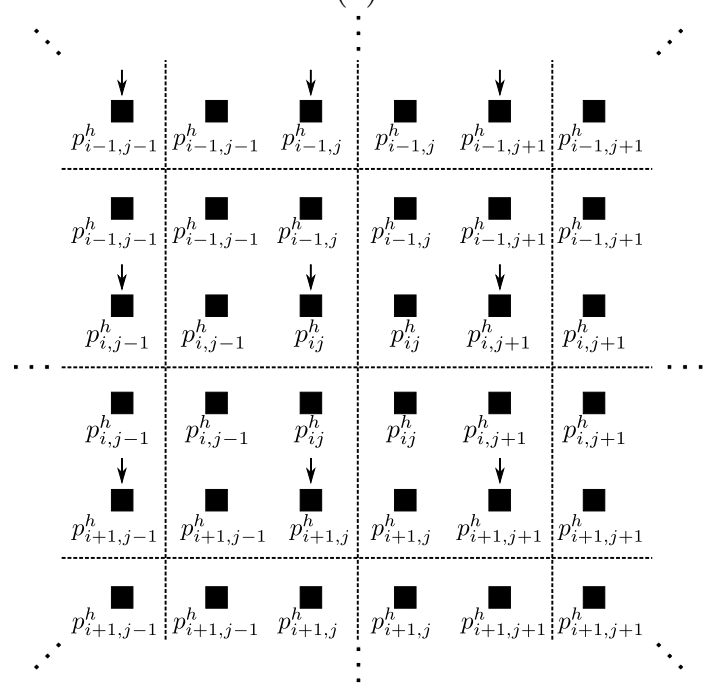

(c)

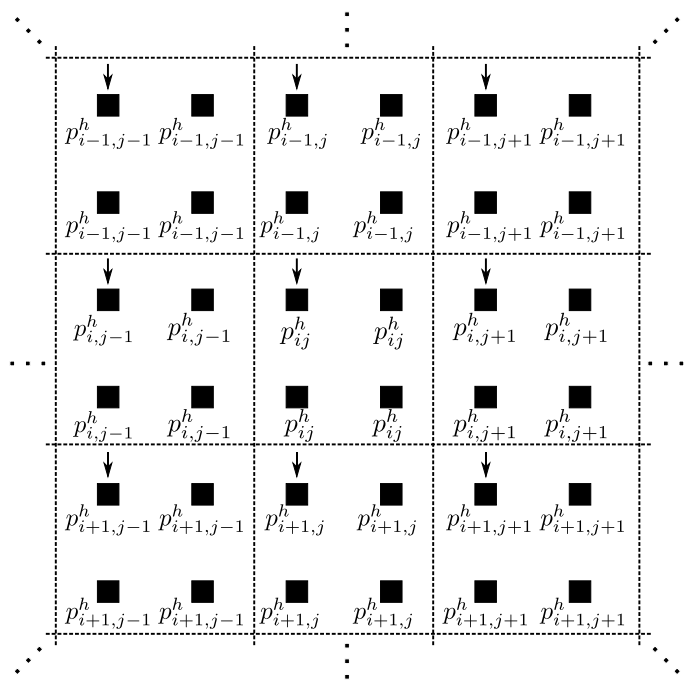

(b)

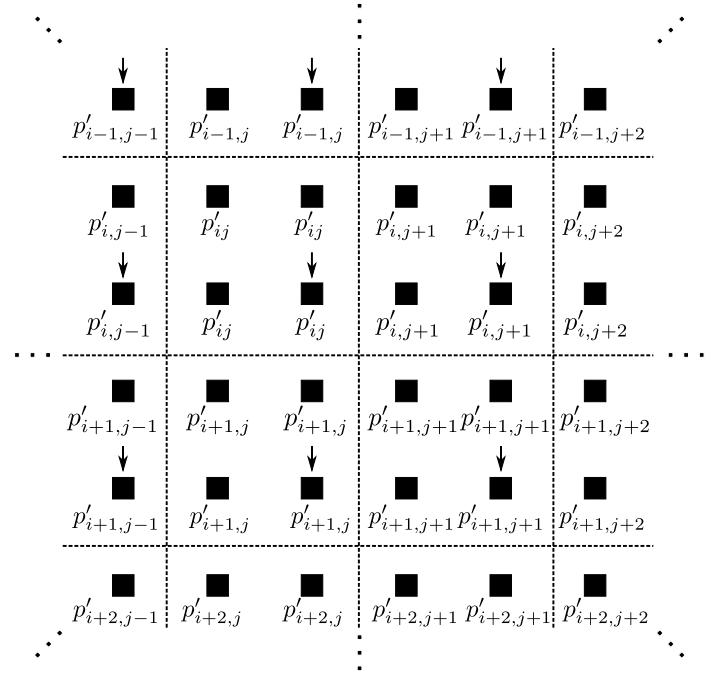

(d)

FIGURE 3.8: Multiresolusion filtering: (a) Image plane division into $2 \times 2$-px blocks; (b) Averaging within every block; (c) Redefinition of the division grid; (d) Averaging with the new configuration.

\subsection{Image pre-distortion for reduced kernel filtering ${ }^{1}$}

Consider a pixel array divided into $2 \times 2$-px blocks, as depicted in Fig. 3.8(a). Diffusion takes place within each block and, eventually, for a long enough time interval, averaging is obtained. The resulting state is depicted in Fig. 3.8(b), where all four pixels of every block are labeled with the same value corresponding to their mean:

$$
p_{i j}^{h}=\frac{1}{4}\left(p_{i, j}+p_{i, j+1}+p_{i+1, j}+p_{i+1, j+1}\right)
$$

1 (C)2011 IEEE. Reprinted, with permission, from J. Fernández-Berni, R. Carmona-Galán, Á. RodríguezVázquez, "Image Filtering by Reduced Kernels Exploiting Kernel Structure and Focal-Plane Averaging", 20th European Conference on Circuit Theory and Design, pp. 229-232, Linköping, Sweden, August 29-31, 2011. 
Small arrows point to the pixels to be sampled in order to obtain an image which will be a quarter of the size of the original full-resolution image, i. e. half of its height and half of its width. Let us assume that a $3 \times 3$ kernel of the form:

$$
\mathbf{K}=\frac{1}{4}\left[\begin{array}{ccc}
a & a+b & b \\
a+c & a+b+c+d & b+d \\
c & c+d & d
\end{array}\right]
$$

must be applied to this half-resolution image.

We now redefine the division grid by shifting the edges of the grouping scheme one pixel down and one pixel to the right (Fig. 3.8(c)). Averaging is again performed for the new blocks configured. Thus, the pixels which were originally $p_{i j}^{h}, p_{i, j+1}^{h}, p_{i+1, j}^{h}$ and $p_{i+1, j+1}^{h}$ are now (Fig. 3.8(d)):

$$
\begin{array}{ll}
p_{i j}^{\prime} & =\frac{1}{4}\left(p_{i-1, j-1}^{h}+p_{i-1, j}^{h}+p_{i, j-1}^{h}+p_{i j}^{h}\right) \\
p_{i, j+1}^{\prime} & =\frac{1}{4}\left(p_{i-1, j}^{h}+p_{i-1, j+1}^{h}+p_{i j}^{h}+p_{i, j+1}^{h}\right) \\
p_{i+1, j}^{\prime} & =\frac{1}{4}\left(p_{i, j-1}^{h}+p_{i j}^{h}+p_{i+1, j-1}^{h}+p_{i+1, j}^{h}\right) \\
p_{i+1, j+1}^{\prime} & =\frac{1}{4}\left(p_{i j}^{h}+p_{i, j+1}^{h}+p_{i+1, j}^{h}+p_{i+1, j+1}^{h}\right)
\end{array}
$$

By subsampling just like before shifting the grid, the resulting image will be again quarter-size, i.e. half-resolution. But the point is that the result of applying the reduced kernel:

$$
\mathbf{K}^{\prime}=\left[\begin{array}{ll}
a & b \\
c & d
\end{array}\right]
$$

to the half-resolution image obtained after shifting the grid (Fig. 3.8(d)), being pixel $p_{i j}^{\prime}$ the one weighted by the upper-left element, $a$, is the same as applying the kernel of Eq. (3.17) to the original half-resolution image (Fig. 3.8(b)), centered in $p_{i j}^{h}$. This scheme of pre-processing can be extended to lower image resolutions provided that the size of the blocks is $B \times B$, being $B$ an even number. In this case, the grid must be shifted $B / 2$ pixels to achieve a representation at the corresponding lower resolution for which Eq. (3.19) can be applied.

Although the symmetries demanded by Eq. (3.17) and Eq. (3.19) greatly restrict the number of kernels that can be reduced, some very useful templates in early vision processing coincidentally fall into this category. For instance, the Sobel operators [González \& Woods, 2002], which compute an approximation to the components of the image intensity gradient, are transformed into $2 \times 2$ kernels in the following way: 


$$
\begin{aligned}
\mathbf{G}_{x} & =\left[\begin{array}{rrr}
1 & 0 & -1 \\
2 & 0 & -2 \\
1 & 0 & -1
\end{array}\right] \quad \rightarrow \mathbf{G}_{x}^{\prime}=4\left[\begin{array}{rr}
1 & -1 \\
1 & -1
\end{array}\right] \\
\mathbf{G}_{y} & =\left[\begin{array}{rrr}
1 & 2 & 1 \\
0 & 0 & 0 \\
-1 & -2 & -1
\end{array}\right] \rightarrow \mathbf{G}_{y}^{\prime}=4\left[\begin{array}{rr}
1 & 1 \\
-1 & -1
\end{array}\right]
\end{aligned}
$$

where $\mathbf{G}_{x}$ approximates the derivative in the horizontal direction while $\mathbf{G}_{y}$ approximates the derivative in the vertical direction.

Other interesting reducible template is the $3 \times 3$ binomial kernel [Jahne, 2001].

$$
\mathbf{G}_{b}=\frac{1}{16}\left[\begin{array}{ccc}
1 & 2 & 1 \\
2 & 4 & 2 \\
1 & 2 & 1
\end{array}\right] \rightarrow \mathbf{G}_{b}^{\prime}=\frac{1}{4}\left[\begin{array}{cc}
1 & 1 \\
1 & 1
\end{array}\right]
$$

Note that we can even complete this filtering by simply shifting the division grid back to its original position and realizing once more a complete diffusion within the resulting blocks. These additional steps, depicted in Fig. 3.9, lead to a half-resolution filtered image which is directly equivalent to apply $\mathbf{G}_{b}$ to the original half-resolution image represented by Fig. 3.8(b). Furthermore, this image (Fig. 3.9(b)) could be again filtered by repeating the sequence of shifting the division grid as just described so many times as necessary. Thus, for $n$ repetitions, the resulting image will be equivalent to filter the half-resolution image of Fig. 3.8(b) $n$ times with the $3 \times 3$ binomial kernel. As this kernel is a spatially-discretized truncated version of a continuous Gaussian filter with $\sigma^{2}=0.5$, this operation is enabling the generation of multiresolution scale spaces.

\subsection{Energy-based scene representation}

Another interesting processing primitive based on diffusion and reconfigurable image plane division is an energy-based scene representation. According to Eq. (3.13), the energy of each frequency component during the diffusion can be expressed as:

$$
\left|\hat{V}_{u v}(t)\right|^{2}=\left|\hat{V}_{u v}(0)\right|^{2} e^{-8 D t\left[\sin ^{2}\left(\frac{\pi u}{M}\right)+\sin ^{2}\left(\frac{\pi v}{N}\right)\right]}
$$

being the total energy of $V_{i j}$ at the time instant $t$ : 


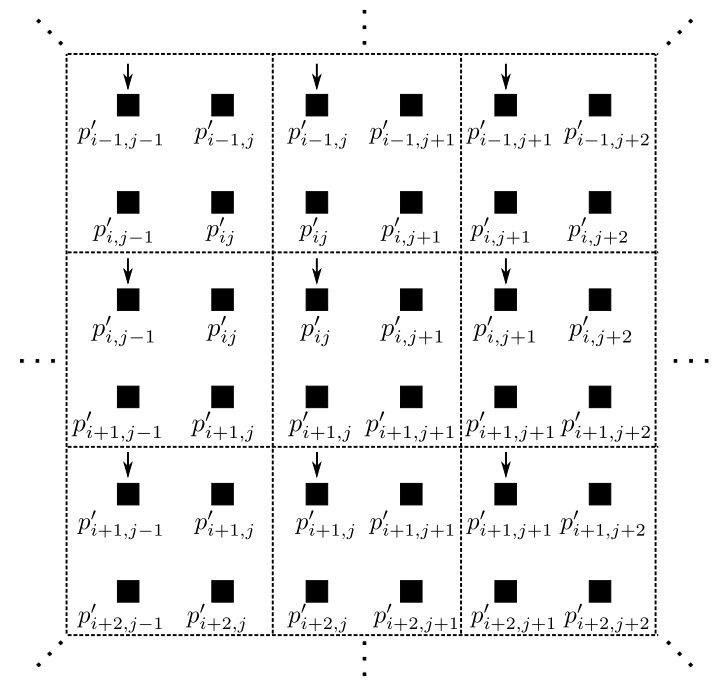

(a)

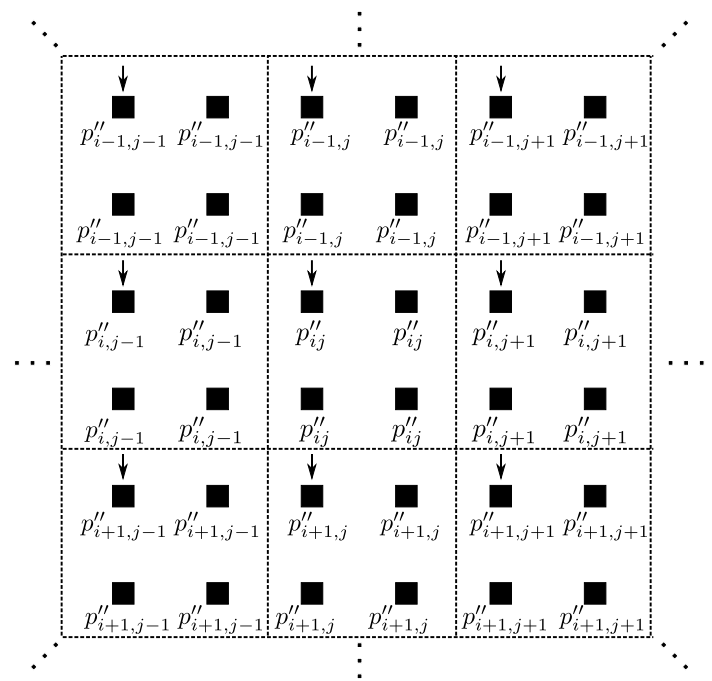

(b)

Figure 3.9: Multiresolution filtering: (a) Grid division of Fig. 3.8(d) shifted back to its original position; (b) Averaging within every block in order to achieve binomial filtering.

$$
E_{T}(t)=\sum_{u=0}^{M-1} \sum_{v=0}^{N-1}\left|\hat{V}_{u v}(t)\right|^{2}
$$

From this equation, we can conclude that the single value of the total energy along time accounts for the filtering undergone during the diffusion. The longer $t$ the less $E_{T}(t)$. The energy lost between two time instants during the diffusion corresponds to that of the isotropic spatial frequencies filtered whereas the energy remaining at the end of the diffusion is associated exclusively with the dc component. Consequently, $E_{T}(t)$ can be used as an indicator of the frequency content of $V_{i j}$. The total energy can be also expressed in the spatial domain as follows:

$$
E_{T}(t)=\sum_{i=1}^{M} \sum_{j=1}^{N}\left|V_{i j}(t)\right|^{2}
$$

As an example of application of this primitive, consider Fig. 3.10. The objective is to simplify the tracking of a flock of birds (Fig. 3.10(a)) by finding a measure of the number of birds contained in a certain region of the image. To this end, a regular block-wise division is applied (Fig. 3.10(b)). Let us assume that linear diffusion is carried out within each block, indexed by $k$ and $l$. The values of the energy at the beginning, $E_{T_{k l}}(0)$, and at the end, $E_{T_{k l}}(\infty)$, of the diffusion are extracted for every block, whose value will be given by the following expression:

$$
B_{k l}=\frac{E_{T_{k l}}(0)-E_{T_{k l}}(\infty)}{E_{T_{k l}}(0)}
$$

That is to say, each block is defined as the quotient of its total energy, $E_{T_{k l}}(0)$, neglecting its dc component $E_{T_{k l}}(\infty)$, and its total energy but now including this de component. This is a 


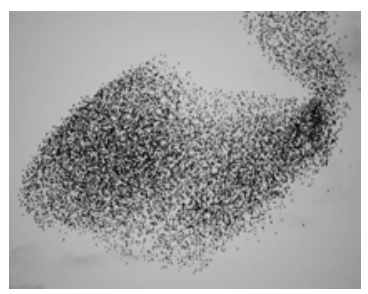

(a)

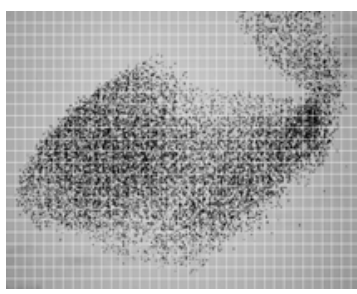

(b)

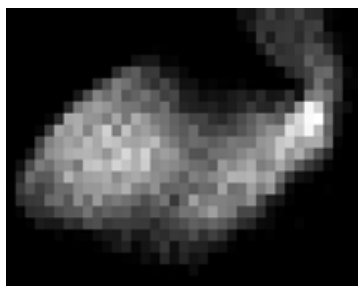

(c)

FIGURE 3.10: Example of energy-based simplification of a scene: (a) Original image containing a flock of birds; (b) Block-wise division; (c) Result of computing Eq. (3.25) within every block and normalizing.

spatial highpass filter normalized to the total energy associated with the corresponding block. The outcome, depicted in Fig. 3.10(c), is an estimation of the bird density at a coarser grain than the full-size image, making thus pixel-level analysis unnecessary.

\subsection{Summary}

This chapter sets out the mathematical framework related to image processing primitives capable of generating different degrees of simplification of a scene. These primitives can be summarized as follows:

- Progressive spatial filtering and correlated subsampling. A time-controlled diffusion process applied to an image enables its analysis on different spatial frequencies and permits scale space, foveation and Gaussian pyramid generation.

- Fully-programmable multiresolution scene representation. Different resolutions can be obtained by grouping pixels in rectangular-shape size-variant user-defined blocks, letting diffusion finish within each block, that is, averaging, and subsampling one pixel out of every block.

- Reduced kernel filtering. The sequence 'shifting and averaging' over a prescribed pixel grouping scheme outputs a pre-processed version of the image which simplifies the subsequent application of convolution kernels meeting certain symmetries.

- Block-wise energy-based scene representation. This primitive, combined with the progressive spatial filtering of diffusion, allows to efficiently segment spatially-repetitive patterns and high contrast zones at different scales within the scene. 
Now, it is time to address the other major issue concerning the incorporation of vision to WSN nodes: a power-efficient VLSI implementation of these primitives. As a result, we will achieve to reduce the data flow representing an image sequence at very low energy cost. 



\section{Chapter 4}

\section{VLSI implementation of linear}

\section{diffusion}

\subsection{Introduction}

Linear diffusion is the cornerstone on which the primitives defined in the previous chapter are built. Its most prominent feature, in terms of image processing, is the programmability of its inherent Gaussian filtering. Indeed, the VLSI implementation of user-defined image Gaussian filtering has been already explored in the literature. Most of the approaches reported make use of the ability of CMOS processes to integrate signal processing circuitry with pure imaging. Thus, they achieve massively parallel focal-plane processing, delivering the same result as a digital processor but in a much more efficient way.

Resistive grids are the first option to be considered [Mead, 1989; Raffo et al., 1998]. They can perform spatial filtering and are mainly composed of passive elements. If necessary, negative resistors could be also incorporated. Their robustness to mismatch makes them specially suitable for VLSI implementation [Hui \& Shi, 1999; Shi, 2009]. In [Kobayashi et al., 1991], a singlechip analog implementation of a resistive network for Gaussian filtering is described. Negative resistors are mandatory in order to attain a Gaussian-like convolution kernel, what makes the circuitry bulky due to the negative impedance converters. The variation of the filter width is achieved by two MOSFETs in parallel biased in the triode region whose control of the gate voltages results in a variable resistor. It is precisely the control circuit of this variable resistor what greatly increases the power consumption of the chip. Moreover, only Gaussians with a width variable by a factor of 2 are available. Other possibility of Gaussian filtering in resistive grids is through MOSFETs working in subthreshold regime [Vittoz \& Arreguit, 1993; Andreou 
\& Boahen, 1995]. In this case, the main drawback is the significant influence of leakage currents and mismatch for a fine control of the filtering width [Leñero et al., 2009].

The widely known CNN framework [Chua \& Roska, 1993] can theoretically emulate the filtering performed by linear diffusion [Shi \& Chua, 1992]. However, we will demonstrate in this chapter that the unavoidable mismatch presents at any VLSI implementation prevents typical transconductor-based approach from achieving Gaussian filtering with enough accuracy, specially for large widths. We are going to find the evidence of this claim by studying unconstrained diffusion in CNN implementations. A simple 2-cell network will suffice to point out the damaging effect of mismatch in the position of the natural frequencies of the network. Simulations on larger networks will confirm the extrapolation of these results. Then, we will point out how linear diffusion can be constrained in $\mathrm{CNN}$ hardware, establishing the equivalence between a constrained diffusion and stopping the unconstrained diffusion at a specific time instant. We will find that the effect of mismatch is similar in both cases.

In [Ni et al., 1993], the physical implementation of Gaussian filters with user-defined width is addressed in a totally different way. It introduces a capacitive network which can be considered as a numeric solver of the spatially-discretized diffusion equation. The variance of the filter is determined by a capacitor ratio, fixed by layout design, and an iteration number associated to the implicit time discretization of the network. Four switches, two switching capacitors and one grounded capacitor amount to each node. An error of $1 \%$ is delivered when the iteration number is higher than ten. A more recent VLSI implementation of this approach is reported in $[\mathrm{Ni}$, 2005].

Finally, we propose in this chapter to take advantage of the dynamics of an RC network in order to achieve user-defined Gaussian filters. This dynamics consists of a real diffusion itself. The initial charge at the capacitors is redistributed through a diffusion process whose pace is determined by the time constant of the network. Thus, an RC network can be also considered as a solver of the spatially-discretized diffusion equation, but no time discretization is now realized. This means that any filter width is ideally possible. The main drawback which can be argued against this approach is the considerable area consumption of resistors in CMOS processes. We will demonstrate that this problem can be solved by substituting each resistor by a single MOS transistor biased in the ohmic region. The sheet resistance of the MOS channel is much greater than that of the resistors made with polysilicon or diffusion strips. Besides, the dynamics of the network can be activated or deactivated by controlling the gate voltages of the transistors. The effects of the inevitable nonlinearities introduced by the MOSFETs are alleviated by a careful design of the elementary transistor. An error below $1 \%$ is thus achieved, which is translated into perceptually equivalent outputs. Only two transistors, acting as switches with an ON resistance designed ad-hoc, and a grounded capacitor amount to each node. 


\subsection{Linear diffusion in transconductance-based $\mathrm{CNNs}^{1}$}

\subsubsection{Unconstrained linear diffusion in CNNs}

\subsubsection{Mapping of the diffusion equation into the CNN grid}

Our starting point is Eq. (3.10). Making ${ }^{2} \tau=D^{-1}$, it is easy to see that this equation describes a CNN whose states follow:

$$
\tau \frac{d V_{i j}}{d t}=-g\left(V_{i j}\right)+\sum_{k=-1}^{1} \sum_{l=-1}^{1} a_{k l} V_{i+k, j+l}+b_{k l} u_{i+k, j+l}+z
$$

with this set of templates:

$$
A=\left[\begin{array}{ccc}
0 & 1 & 0 \\
1 & -4 & 1 \\
0 & 1 & 0
\end{array}\right], B=\left[\begin{array}{lll}
0 & 0 & 0 \\
0 & 0 & 0 \\
0 & 0 & 0
\end{array}\right], z=0
$$

and where the losses term, introduced in the full-signal-range (FSR) CNN model [Espejo et al., 1996], has the form:

$$
g\left(V_{i j}\right)=\lim _{m \rightarrow \infty}\left\{\begin{array}{llr}
m\left(V_{i j}-1\right) & \text { if } & V_{i j}>1 \\
0 & \text { if } & \left|V_{i j}\right| \leq 1 \\
m\left(V_{i j}+1\right) & \text { if } & V_{i j}<-1
\end{array}\right.
$$

The value of the original losses resistor in Chua-Yang's model has been incorporated into the central element of the feedback template. This is common in VLSI implementation of CNNs as it reduces the number of circuit elements at each cell. Fig. 4.1 depicts a node of the already described CNN, where the voltage limitation imposed by $g\left(V_{i j}\right)$ is implemented by ideal diodes with $E_{S}=1 \mathrm{~V}$. The time constant is given by $\tau=C / g_{m}$. As can be seen, the contributions of the neighboring cells are conveyed in the form of current via transconductances accurately matched.

\subsubsection{State equations}

Consider the bidimensional CNN in Fig. 4.2. It is composed by $M \times N$ cells, each following the evolution law expressed by Eq. (4.1) with the templates in Eq. (4.2). In order to analyze the network dynamics, we would like to describe it as a matrix differential equation like this:

\footnotetext{
1 (C)2009 Wiley. Reprinted, with permission, from J. Fernández-Berni, R. Carmona-Galán, "On the Implementation of Linear Diffusion in Transconductance-Based Cellular Nonlinear Networks". International Journal of Circuit Theory and Applications, Vol. 37, No. 4, pp. 543-567, May 2009.

${ }^{2}$ This equality contemplates the numerical values of $\tau$ and $D$, although they have different dimensions
} 


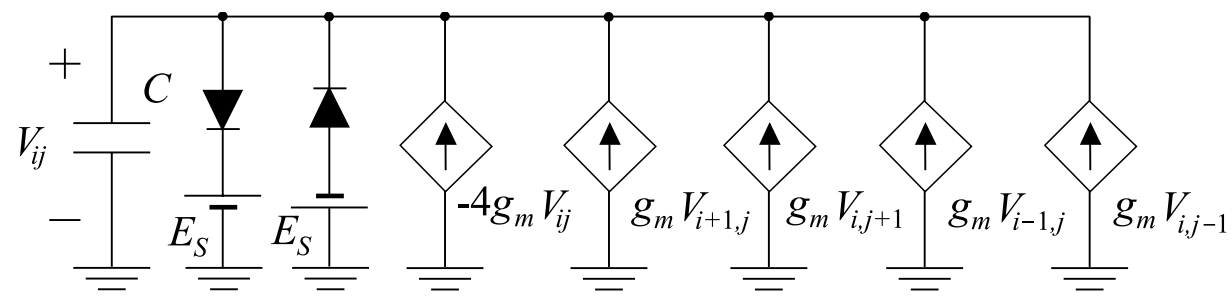

FiguRE 4.1: Processing node of a CNN implementing unconstrained linear diffusion

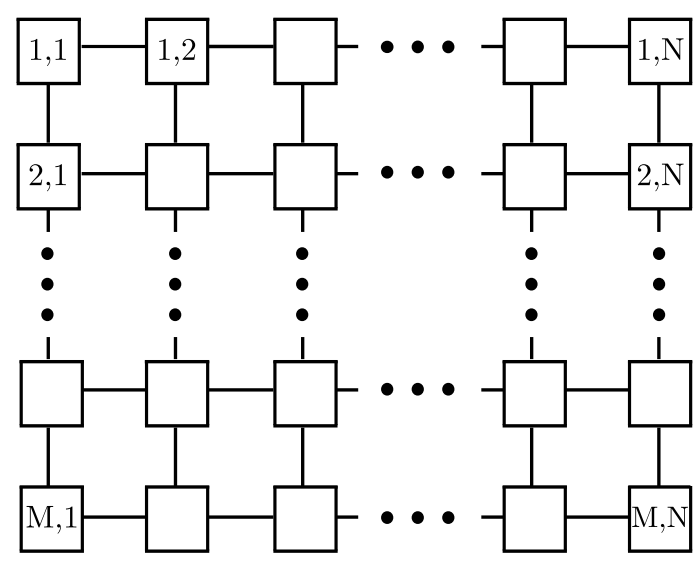

Figure 4.2: Bidimensional CNN composed by $M \times N$ cells carrying out unconstrained linear diffusion

$$
\dot{\mathbf{V}}(t)=\mathbf{M V}(t)
$$

Notice that the system is homogeneous, as the diffusion evolves from the initial state without any external forcing agent, until reaching the equilibrium point. That is to say, an unconstrained linear diffusion is carried out. In this equation, $\mathbf{V}(t)$ is a single column vector in which we group the state variables of all the cells:

$$
\mathbf{V}(t)=\left[\begin{array}{lllllll}
V_{11}(t) & \ldots & V_{1 N}(t) & V_{21}(t) & \ldots & V_{M, N-1}(t) & V_{M N}(t)
\end{array}\right]^{T}
$$

This simplified notation is commonly used in literature [Chua \& Roska, 2002]. Following this scheme, $\mathbf{M}$ is the system matrix, and contains the $M N \times M N$ coefficients that weigh the contributions of the states of each cell in the grid into the evolution of every other cell. This matrix is defined by: 


$$
\mathbf{M}=\left[\begin{array}{cccccccc}
m_{11 ; 11} & \cdots & m_{11 ; 1 N} & m_{11 ; 21} & \cdots & m_{11 ; 2 N} & \cdots & m_{11 ; M N} \\
m_{12 ; 11} & \cdots & m_{12 ; 1 N} & m_{12 ; 21} & \cdots & m_{12 ; 2 N} & \cdots & m_{12 ; M N} \\
\vdots & & \vdots & \vdots & & \vdots & & \vdots \\
m_{1 N ; 11} & \cdots & m_{1 N ; 1 N} & m_{1 N ; 21} & \cdots & m_{1 N ; 2 N} & \cdots & m_{1 N ; M N} \\
m_{21 ; 11} & \cdots & m_{21 ; 1 N} & m_{21 ; 21} & \cdots & m_{21 ; 2 N} & \cdots & m_{21 ; M N} \\
\vdots & & \vdots & \vdots & & \vdots & & \vdots \\
m_{M N ; 11} & \cdots & m_{M N ; 1 N} & m_{M N ; 21} & \cdots & m_{M N ; 2 N} & \cdots & m_{M N ; M N}
\end{array}\right]
$$

Here, each $m_{i j ; k l}$ indicates the weight of the state of the cell at node $(k, l)$ of the grid in the linear differential equation describing the evolution of the state variable of the cell at $(i, j)$. The values of these coefficients are:

$$
m_{i j ; k l}=\left\{\begin{array}{ccc}
-c / \tau & \text { if } \quad(i, j)=(k, l) \\
1 / \tau & \text { if } & (i, j) \neq(k, l) \text { and the cells are connected } \\
0 & \text { otherwise } &
\end{array}\right\}
$$

where $c$ is the number of neighboring cells connected to cell $(i, j)$. From Fig. 4.2, $c=4$ for a cell inside the array, $c=3$ for a cell on a side and $c=2$ for a cell on a corner. If $\mathbf{M}$ can be diagonalized, a set of $M N$ eigenvalues, $\zeta_{k}$, and the associated eigenvectors, $\mathbf{u}_{k}$, can be obtained. The state of the cells is described by a combination of exponentials of the eigenvalues weighted by their associated eigenvectors:

$$
\mathbf{V}(t)=\sum_{k=0}^{M N-1} c_{k} \mathbf{u}_{k} e^{\zeta_{k} t}
$$

The constants $c_{0}, c_{1}, \ldots, c_{M N-1}$ are determined by the initial conditions. Concerning the stability of the network, the real part of each $\zeta_{k}$ should not be positive. Fig. 4.3 displays the eigenvalues corresponding to square grids of increasing sizes. Here, all of the system matrices are diagonalizable. Their eigenvalues are real. Another important aspect is that all of the eigenvalues are negative but one, let us call it $\zeta_{0}$, which is equal to zero. According to Eq. (4.8), these networks converge. After a certain period, the exponentials vanish and the final state will be determined by $c_{0}$ and $\mathbf{u}_{0}$, or, in other words, by the initial conditions.

More observations on Fig. 4.3: the larger the size of the network, the more eigenvalues concentrate in the vicinity of 0 . As we will see, it means that the larger the network, the more probable is to have a positive eigenvalue if mismatch is taken into account. Another issue: all the eigenvalues belong to the interval $[-8 / \tau, 0]$. We have not found a reason for that but it could be related to the particular connectivity pattern implemented in the network. 


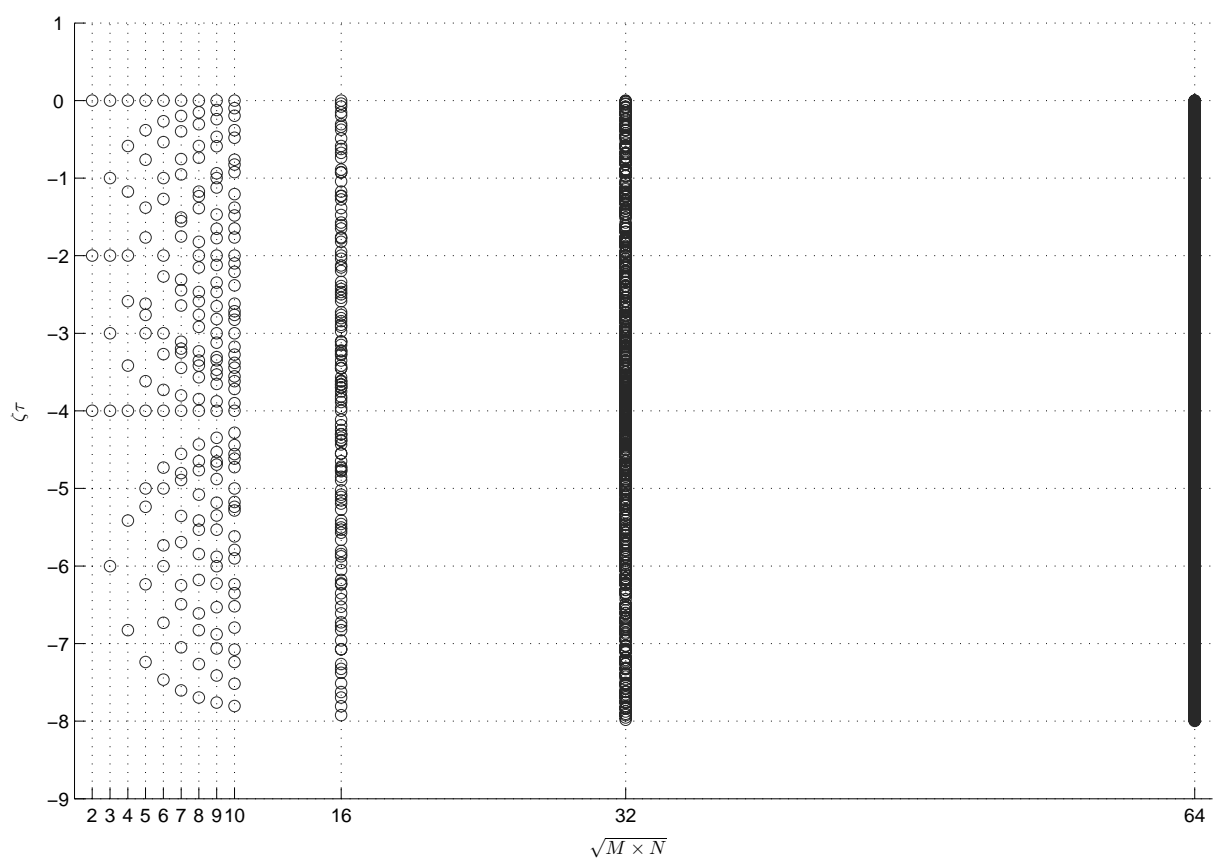

FiguRE 4.3: Eigenvalues for several ideal square CNNs implementing unconstrained diffusion
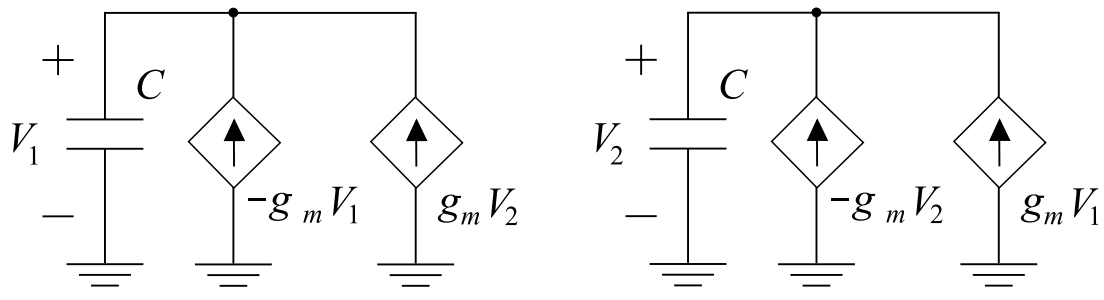

FiguRE 4.4: 2-cell CNN programmed for unconstrained diffusion

\subsubsection{Effect of mismatch: analysis of a 2-cell CNN}

In order to observe the effect of mismatches on CNN hardware implementing unconstrained linear diffusion, consider now the circuit in Fig. 4.4. It is the elementary unidimensional version of a CNN for unconstrained linear diffusion. We are considering that the losses term has been incorporated into the central element of the feedback template. The limiters of the state variables have been excluded from the diagram because all the action takes place within the linear region. The matrix equation describing the network behavior is:

$$
\left[\begin{array}{c}
\dot{V}_{1} \\
\dot{V}_{2}
\end{array}\right]=\frac{1}{\tau}\left[\begin{array}{rr}
-1 & 1 \\
1 & -1
\end{array}\right]\left[\begin{array}{l}
V_{1} \\
V_{2}
\end{array}\right]
$$

where, as before, $\tau=C / g_{m}$. The eigenvalues, and thus the frequencies of the natural response of the system, are: 


$$
\zeta_{0}=0 \quad \zeta_{1}=-2 / \tau
$$

As expected, one eigenvalue equals zero and the other is negative. This dynamics leads to equilibrium following:

$$
\left[\begin{array}{l}
V_{1}(t) \\
V_{2}(t)
\end{array}\right]=\frac{1}{2}\left(E_{1}+E_{2}\right)\left[\begin{array}{l}
1 \\
1
\end{array}\right]+\frac{1}{2}\left(E_{1}-E_{2}\right)\left[\begin{array}{r}
1 \\
-1
\end{array}\right] e^{-2 t / \tau}
$$

The steady state is then found at the average of the initial values:

$$
V_{1}(\infty)=V_{2}(\infty)=\frac{E_{1}+E_{2}}{2}
$$

Now, let us suppose that the transconductance generating the contribution, for instance, from cell 2 to cell 1 has slightly moved away from its ideal value. Let us consider a small deviation $\Delta g_{m}$ that, for simplicity in the notation, we are going to consider to be $\delta$ times $g_{m}$. Now, the equation describing the system is:

$$
\left[\begin{array}{c}
\dot{V}_{1} \\
\dot{V}_{2}
\end{array}\right]=\frac{1}{\tau}\left[\begin{array}{cc}
-1 & 1+\delta \\
1 & -1
\end{array}\right]\left[\begin{array}{l}
V_{1} \\
V_{2}
\end{array}\right]
$$

Keep in mind that this deviation, $\delta$, could be, in principle, either positive or negative. The eigenvalues are now:

$$
\zeta_{0}=-\frac{1}{\tau}(1-\sqrt{1+\delta}) \quad \zeta_{1}=-\frac{1}{\tau}(1+\sqrt{1+\delta})
$$

and thus the state variables of the cells follow:

$$
\left[\begin{array}{c}
V_{1}(t) \\
V_{2}(t)
\end{array}\right]=\frac{1}{2}\left(E_{1}+E_{2} \sqrt{1+\delta}\right)\left[\begin{array}{c}
1 \\
\frac{1}{\sqrt{1+\delta}}
\end{array}\right] e^{\zeta_{0} t}+\frac{1}{2}\left(E_{1}-E_{2} \sqrt{1+\delta}\right)\left[\begin{array}{c}
1 \\
-\frac{1}{\sqrt{1+\delta}}
\end{array}\right] e^{\zeta_{1} t}
$$

It can be observed that if $\delta>0$, one of the eigenvalues, $\zeta_{0}$, turns to be positive. This means that even the slightest deviation with regard to the ideal value — if it is unfortunately positive - can significantly modify the dynamics of the network, because the network diverges and cells end up in saturation. If $\delta<0$, the eigenvalues are either real and negative, as long as $\delta \geq-1$, or conjugate complex with negative real parts, if $\delta<-1$. In both cases, the exponentials in Eq. (4.15) vanish with time, and the equilibrium point becomes: 


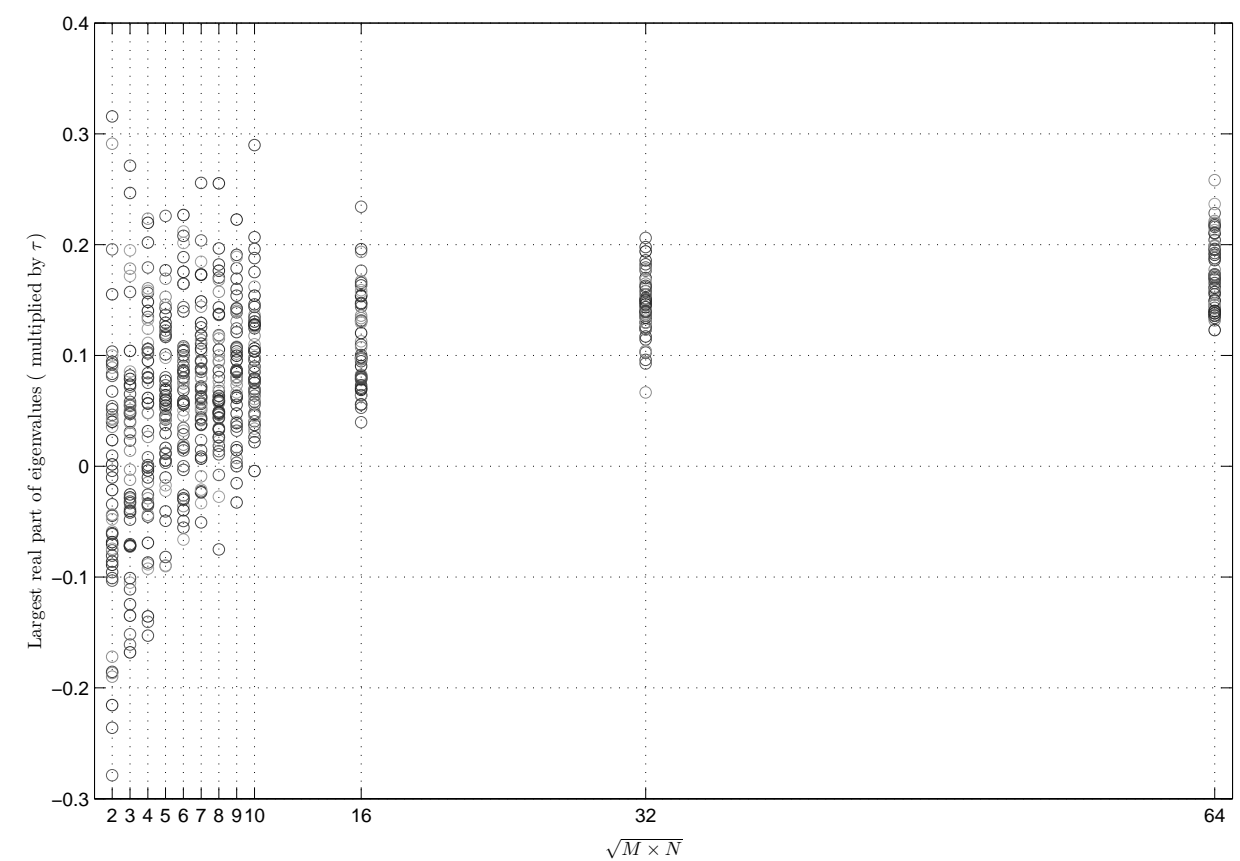

Figure 4.5: Largest real part of eigenvalues for several CNNs implementing unconstrained diffusion and suffering a $\sigma=10 \%$ mismatch

$$
V_{1}(\infty)=V_{2}(\infty)=0
$$

what is clearly not the correct outcome of the unconstrained diffusion of the initial states. Therefore, any $\delta \neq 0$ has a catastrophic influence on the result. As mismatch is inherent to physical realizations, these results point out that a CNN based on transconductances is unable, in practice, to reach the correct final steady-state of an unconstrained linear diffusion.

\subsubsection{Numerical extrapolation of the results}

In order to extend the validity of these results to larger networks, we computed the eigenvalues for square grids of increasing sizes. In this case, the transconductances could vary from the nominal value following a Gaussian distribution with $\sigma=10 \%$. We realized 50 iterations for each size, obtaining 50 sets of eigenvalues. These eigenvalues deviate from the nominal values, depicted in Fig. 4.3, because of the dissimilarities introduced by mismatch. A qualitative analysis of the effect in the dynamics of the network can be extracted by studying the real parts of these eigenvalues. Fig. 4.5 shows the largest real part in each of the 50 sets of eigenvalues. All the rest within the set have a real part below that plotted in the graph. Several things can be observed. First, the larger the grid size, the more the eigenvalues with positive real parts found. In other words, the larger the grid, the more probable is to have a deviation that leads to non-convergence. 

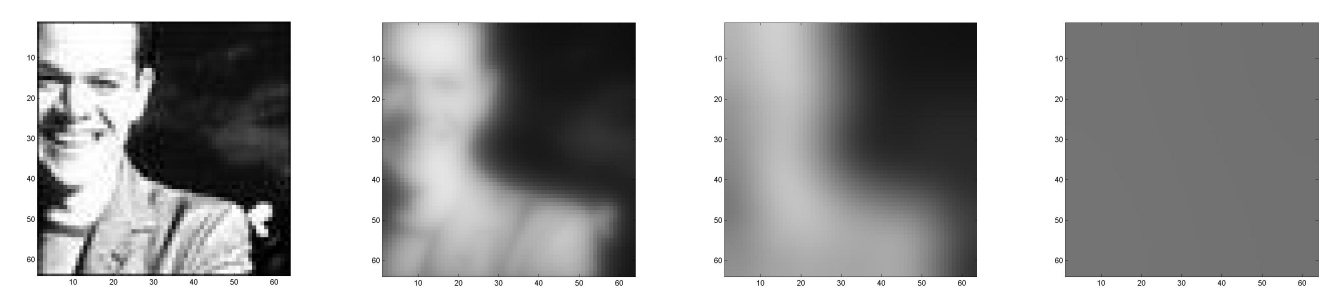

Figure 4.6: Ideal CNN implementing unconstrained linear diffusion: state variables at different time instants along the diffusion
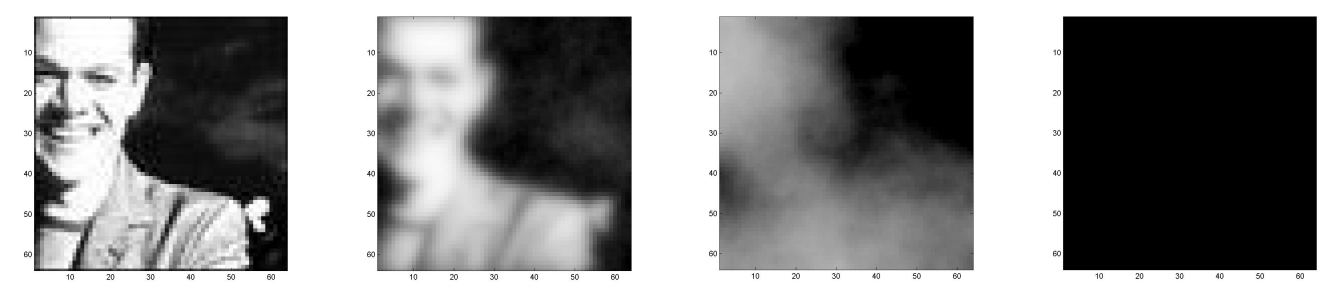

Figure 4.7: CNN implementing unconstrained linear diffusion with $\sigma=1 \%$ : state variables at successive time instants

Second, the larger the grid size, the less influence deviations have in the rest of the cells, i. e. the weaker the components of the eigenvectors relating distant cells. This explains the initial spreading of points in the part of the graph corresponding to smaller networks.

In order to visualize the effect of mismatch in the evolution of the diffusion, we implemented a $64 \times 64$-cell FSR-CNN with ideal linear transconductors in HSPICE. The nominal values for them are $g_{m}=1 \mathrm{mS}$. The network is initialized to the pixel values of the input image scaled and shifted to cover the interval $[-1,1]$. Fig. 4.6 constitutes the reference image sequence. It depicts the state of the cells in an ideal network where the transconductors are perfectly matched at $0 \tau, 12.5 \tau, 31 \tau$ and $1550 \tau$, respectively. It can be observed that the linear diffusion is perfectly carried out. The last image represents the average value of the initial states of the pixels. If we allow each transconductance to deviate from its nominal value, then the diffusion becomes unruly and the network does not converge. Fig. 4.7 depicts the state of the cells at $0 \tau, 3 \tau, 100 \tau$ and $800 \tau$, respectively, when the values of the transconductors follow a Gaussian distribution with $\sigma=1 \%$. For a larger deviation, things get worse, as shown in Fig. 4.8, where the evolution of the RMSE, normalized to the signal range, with regard to the ideal unconstrained diffusion of the same image is depicted for $\sigma=1 \%, \sigma=5 \%$ and $\sigma=10 \%$. The greater the deviation, the larger the positive real parts for the eigenvalues of the network and, therefore, the faster the saturation rails are hit by the cells. The RMSE reaches the same final value in every case because all the cells end up saturating for each value of $\sigma$ whereas the ideal network stabilizes at the average value of the pixels. 


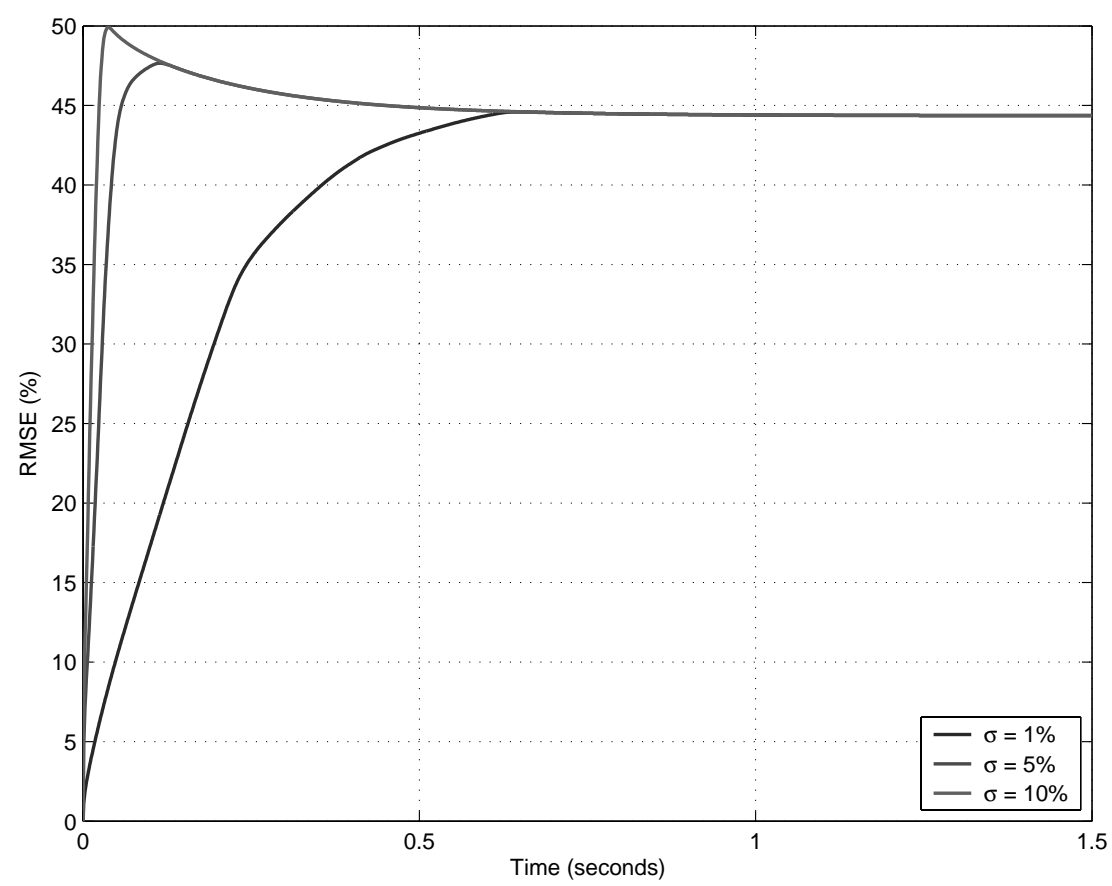

FiguRE 4.8: Evolution of the RMSE with respect to the ideal unconstrained diffusion $(\tau=$ $1 m s)$

These results, obtained by simulation, contradict previous studies on the influence of mismatch in transconductance-based CNNs [Hui \& Shi, 1999]. The source of the discrepancy is, in our view, the initial assumption that small deviations of the network parameters conduct to small deviations from the ideal output. In [Hui \& Shi, 1999], the parameter deviations are translated into equivalent noise sources. The response of the network is then computed by perturbative analysis, and hence the output deviates slightly from the ideal, but, in essence, is of the same nature. As explained previously, the singularity of the location of the eigenvalues, even in a network of only two cells, makes the network extremely sensitive to it. The slightest deviation can move the network dynamics from convergence to non-convergence. In addition, it is worth mentioning that all the natural frequencies of the network, because of the indirect coupling via the $A$-template, contribute to the state variable of every single cell. If one of them is positive, then there is always a point in time after which the contribution of the positive root is far more important than all the rest, thus leading the cells to saturation. In other words, given enough time, all the cells in the network will end up hitting the saturation rails.

\subsubsection{Constrained linear diffusion in CNNs}

\subsubsection{Linear diffusion through a resistive grid}

Let us have a look at a different analog network that can be employed to obtain linear diffusion: a resistive grid. A resistive grid is an association of resistors organized as depicted in Fig. 4.9. 


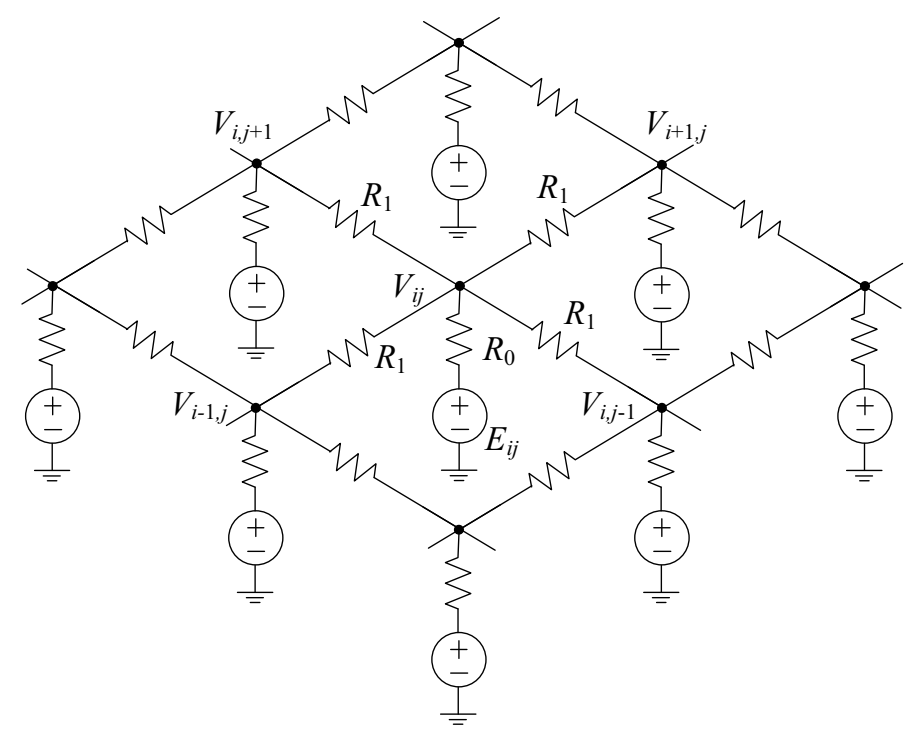

Figure 4.9: Generic resistive grid

If a parasitic capacitance $C_{p}$ is considered to be associated to each node, the evolution of each $V_{i j}$, obviating those at the border of the grid, can be described by:

$$
\tau \frac{d V_{i j}}{d t}=-(4+\lambda) V_{i j}+V_{i+1, j}+V_{i-1, j}+V_{i, j+1}+V_{i, j-1}+\lambda E_{i j}
$$

where $\tau=R_{1} C_{p}$ and $\lambda=R_{1} / R_{0}$ is known as the smoothing factor. The smaller $\lambda$, the stronger the smoothing performed over the input composed by all the $E_{i j}$ 's. In the extreme case in which $\lambda \rightarrow 0$, this equation represents the spatially-discretized unconstrained linear diffusion as in Eq. (3.10). In that case, as there is no input source, the grid becomes an RC network which evolves from the initial conditions, i.e. the initial voltages, at the capacitors.

In order to show that this grid can carry out constrained linear diffusion, let us consider now an elementary resistive grid with $\lambda>0$ (Fig. 4.10(a)). The dynamics of the circuit is described by:

$$
\left[\begin{array}{c}
\dot{V}_{1} \\
\dot{V}_{2}
\end{array}\right]=\frac{1}{R_{1} C_{p}}\left[\begin{array}{cc}
-(1+\lambda) & 1 \\
1 & -(1+\lambda)
\end{array}\right]\left[\begin{array}{l}
V_{1} \\
V_{2}
\end{array}\right]+\frac{\lambda}{R_{1} C_{p}}\left[\begin{array}{c}
E_{1} \\
E_{2}
\end{array}\right]
$$

The steady-state of this circuit is, making $\dot{\mathbf{V}}(t)=0$ :

$$
\mathbf{V}_{P}=\frac{1}{\lambda+2}\left[(1+\lambda) E_{1}+E_{2} \quad E_{1}+(1+\lambda) E_{2}\right]^{T}
$$

By comparing the result of the operation of the resistive grid, Eq. (4.19), to the evolution of the state variables during an unconstrained linear diffusion, Eq. (4.11), we can find a time instant: 


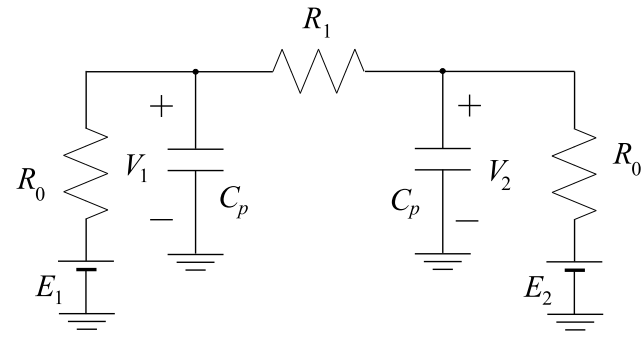

(a)

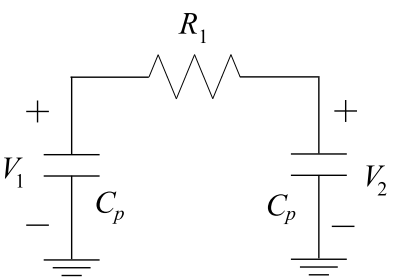

(b)

Figure 4.10: Elementary resistive grid with (a) $\lambda \neq 0$ and (b) resulting RC network for $\lambda=0$

$$
t_{e q}=\frac{\tau}{2} \ln \left(\frac{\lambda+2}{\lambda}\right)
$$

for which an unconstrained linear diffusion stopped at $t_{e q}$ has reached the values that a linear resistive grid with $\lambda=R_{1} / R_{0}$ reaches at steady state.

In addition, from Eq. (4.20) we can see that $\lambda \rightarrow 0$ in the resistive grid results in $t_{e q} \rightarrow \infty$. And also, $\lambda \rightarrow \infty$ results in $t_{e q} \rightarrow 0$, i. e. the diffusion does not start.

\subsubsection{Templates for constrained diffusion}

As reported in [Shi \& Chua, 1992], CNNs are quite capable of implementing resistive grid filtering. For the same CNN model as before, this set of templates:

$$
A=\left[\begin{array}{ccc}
0 & 1 & 0 \\
1 & -(4+\lambda) & 1 \\
0 & 1 & 0
\end{array}\right], B=\left[\begin{array}{lll}
0 & 0 & 0 \\
0 & \lambda & 0 \\
0 & 0 & 0
\end{array}\right], z=0
$$

permits the implementation of a resistive grid with $\lambda>0$. Notice that now the control, or $B$-, template has a central element different from zero.

\subsubsection{Modified state equations}

Now the matrix differential equation describing the network dynamics is inhomogeneous:

$$
\dot{\mathbf{V}}(t)=\mathbf{M}^{\prime} \mathbf{V}(t)+\mathbf{b}
$$

where vector $\mathbf{b}$ contains the inputs to every cell of the array:

$$
\mathbf{b}=\frac{\lambda}{\tau}\left[\begin{array}{lllllll}
E_{11} & \ldots & E_{1 N} & E_{21} & \ldots & E_{M, N-1} & E_{M N}
\end{array}\right]^{T}
$$



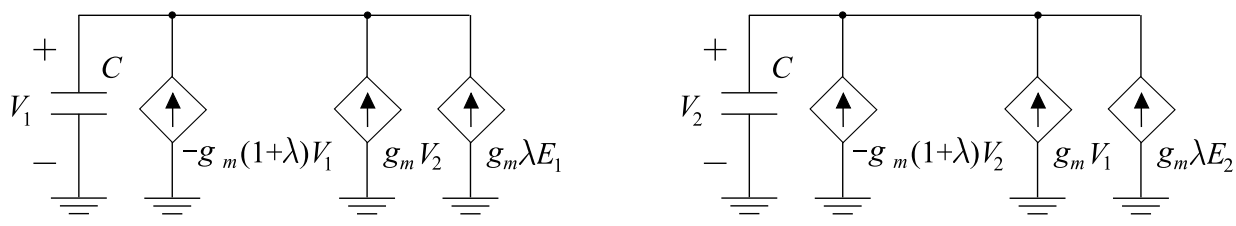

Figure 4.11: 2-cell CNN programmed for constrained diffusion

and the matrix of the coefficients, $\mathbf{M}^{\prime}$, can be obtained from the matrix $\mathbf{M}$ defined by Eq. (4.6) through:

$$
\mathbf{M}^{\prime}=\mathbf{M}-\frac{\lambda}{\tau} \mathbf{I}
$$

being $\mathbf{I}$ the $M N \times M N$ identity matrix. This modified matrix has a different set of eigenvalues, $\zeta_{0}^{\prime}, \zeta_{1}^{\prime}, \ldots, \zeta_{M N-1}^{\prime}$, that is related to the unconstrained linear diffusion set through:

$$
\zeta_{k}^{\prime}=\zeta_{k}-\frac{\lambda}{\tau}
$$

Now, the solution of the inhomogeneous matrix differential equation is the sum of the solution of the homogeneous system plus a particular solution:

$$
\mathbf{V}(t)=\mathbf{V}_{P}(t)+\mathbf{V}_{H}(t)
$$

The particular solution can be obtained by considering $\dot{\mathbf{V}}(t)=0$, then:

$$
\mathbf{V}_{P}=-\mathbf{M}^{\prime-1} \mathbf{b}
$$

For the unconstrained linear diffusion, the largest eigenvalue was $\zeta_{0}=0$. Now it has been transformed into $\zeta_{0}^{\prime}=-\lambda / \tau$. Therefore, all the eigenvalues from $\mathbf{M}^{\prime}$ are real and negative. As a consequence, the solution of the homogeneous system vanishes with time. The steady state is given by the particular solution, obtained from Eq. (4.27).

\subsubsection{Effects of mismatch in a 2-cell CNN with $\lambda>0$}

Consider now the 2-cell CNN programmed to realize constrained linear diffusion (Fig. 4.11). While Eq. (4.18) describes the behavior of this circuit nominally - if we identify $C \equiv C_{p}$ and $g_{m} \equiv 1 / R_{1}$ - the introduction of a slight deviation on the transconductances has consequences in the dynamics of the system. Let us suppose, as before, the simple case in which one of the transconductances weighting the contribution of one cell into the other deviates from the ideal value. The equation describing the system is: 


$$
\left[\begin{array}{l}
\dot{V}_{1} \\
\dot{V}_{2}
\end{array}\right]=\frac{1}{\tau}\left[\begin{array}{cc}
-(1+\lambda) & 1+\delta \\
1 & -(1+\lambda)
\end{array}\right]\left[\begin{array}{c}
V_{1} \\
V_{2}
\end{array}\right]+\frac{\lambda}{\tau}\left[\begin{array}{c}
E_{1} \\
E_{2}
\end{array}\right]
$$

where $\delta$ represents again a deviation that could be positive or negative. The eigenvalues are now:

$$
\zeta_{0}=-\frac{1}{\tau}(1+\lambda-\sqrt{1+\delta}) \quad \zeta_{1}=-\frac{1}{\tau}(1+\lambda+\sqrt{1+\delta})
$$

The real part of both eigenvalues is negative as long as:

$$
1+\lambda \geq \sqrt{1+\delta}
$$

As $\delta$ represents the relative deviation of the value of the transconductance $g_{m}$ from the ideal, the design equation that renders the appropriate forcing of the input to ensure stability and therefore convergence to a steady state is:

$$
\lambda \geq \sqrt{1+\frac{\Delta g_{m}}{g_{m}}}-1
$$

Besides, the steady-state is also affected by the mismatch introduced, causing a deviation from the ideal value in Eq. (4.19):

$$
\mathbf{V}_{P}=\frac{1}{\lambda+2}\left[\begin{array}{c}
(1+\lambda) E_{1}+E_{2} \\
E_{1}+(1+\lambda) E_{2}
\end{array}\right]+\frac{\delta}{(\lambda+2)[\lambda(\lambda+2)-\delta]}\left[\begin{array}{c}
(1+\lambda)\left[E_{1}+(1+\lambda) E_{2}\right] \\
E_{1}+(1+\lambda) E_{2}
\end{array}\right]
$$

from where we can see that the larger $\lambda$, the smaller the error committed but also the shorter the radius of diffusion achieved, that is, the smaller the width of the equivalent Gaussian filter.

\subsubsection{Numerical results for larger networks}

As for the case of unconstrained linear diffusion, we simulated a $64 \times 64$-cell $\mathrm{CNN}$ with ideal linear transconductors whose nominal value was $g_{m}=1 \mathrm{mS}$, being $C=1 \mathrm{pF}$. The capacitors are initialized to 0 volts, the pixels of the image are the inputs of the cells and no mismatch between transconductors is considered. Fig. 4.12 shows the steady state of the pixels. In these pictures, $\lambda$ takes the values $0.25,0.5,0.75$ and 1 , respectively. It can be seen that the smaller $\lambda$ the larger the radius of diffusion. If we allow the transconductances to deviate following a Gaussian distribution with $\sigma=10 \%$, the situation is similar to that of Fig. 4.5 for unconstrained linear diffusion. Fig. 4.13 depicts, in the first place, the steady state for $\lambda=0.1$. All of the cells 
end up in saturation. Following that, the steady state for $\lambda=0.3$ is shown. In this case, only some cells arrive to saturation. From Eq. (4.25) and extrapolating Fig. 4.5, it can be seen that $\lambda=0.3$ is enough to make negative the real part of all the eigenvalues and, therefore, a steady state is reached. However, for $\lambda=0.1$, there is one eigenvalue with positive real part at least, and, therefore, the network diverges. At the same time, $\lambda=0.3$ is still small and it introduces an important deviation in the final value of the state of the cells, some of them falling beyond saturation. For higher $\lambda_{\mathrm{s}}$ (Fig. 4.14) the error is smaller, but the radius of diffusion achieved is shorter. It is numerically demonstrated in Fig. 4.15, where the normalized RMSE with respect to the ideal steady-state is depicted for different values of $\lambda$ and $\sigma$.

\subsubsection{Comparison between time-controlled unconstrained diffusion and constrained diffusion}

In order to compare the evolution of unconstrained and constrained linear diffusion in CNNs under mismatch conditions, we used the same $64 \times 64$-cell FSR-CNN and the same image as before. First of all, with the CNN implementing ideal constrained linear diffusion, we obtained the steady-state for several values of $\lambda$. Then, we looked for the equivalent time instant $t_{e q}$ at which an ideal unconstrained linear diffusion reaches the same radius of diffusion than the corresponding steady-state for each value of $\lambda$. However, the accuracy in the computation of the time instant $t_{e q}$ is limited by the integration step during the simulation, which should be infinitesimally small to find the exact value. In this way, in Table 4.1 showing the results, $t_{e q}$ represents the time instant at which the computed unconstrained linear diffusion using a integration step of $0.01 \tau$ reaches the closest state of diffusion to the corresponding steady-state for each value of $\lambda$.

Using these equivalences, we computed the normalized RMSE with respect to the ideal case from both constrained and time-controlled unconstrained linear diffusion under different mismatch conditions (Fig. 4.16 and Fig. 4.17). As expected, the larger the values of $\lambda$, or, equivalently, the smaller the values of $t_{e q}$, the less the error, that is, the less mismatch affects the outcome. But, the most important aspect to notice here is that, under the same mismatch conditions, both options present similar results. Only in the case $\lambda=0.1$ and $\sigma=10 \%$ there is a significant deviation between the outcomes. However, in practice, both images are too damaged to be used for any processing.

From the point of view of the implementation, constrained linear diffusion does not require an accurate time control for sampling the evolution of the network dynamics, as is the case for unconstrained diffusion. In the constrained case, the radius of diffusion is determined by the smoothing factor, being the steady-state the outcome of the processing. Therefore, it is the best choice. 

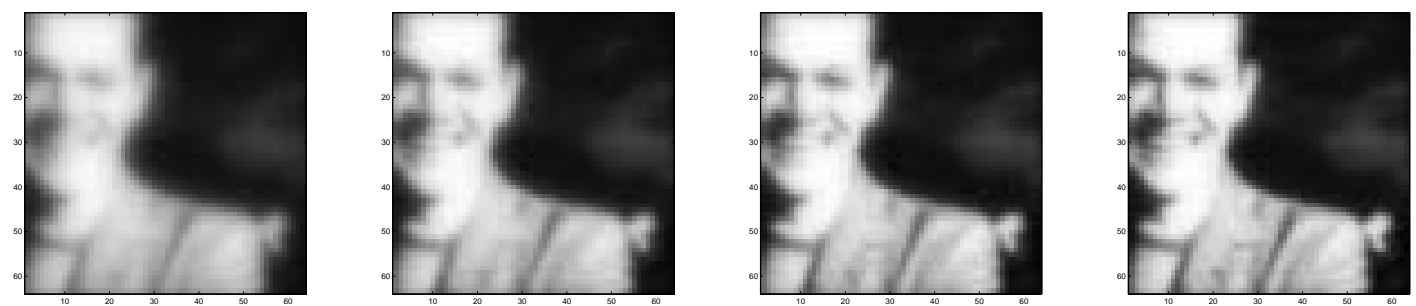

Figure 4.12: Ideal constrained diffusion: steady state for $\lambda=0.25, \lambda=0.5, \lambda=0.75$ and $\lambda=1$ respectively
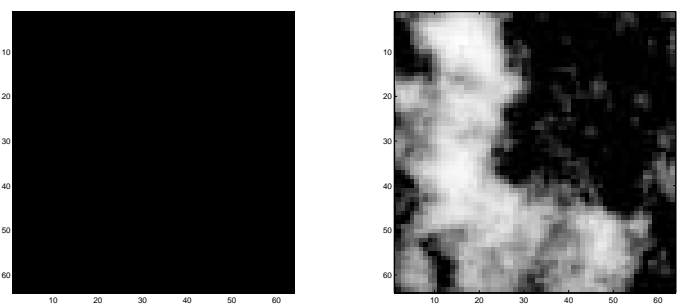

Figure 4.13: Constrained diffusion: steady state for $\lambda=0.1$ and $\lambda=0.3$ respectively with $\sigma=10 \%$
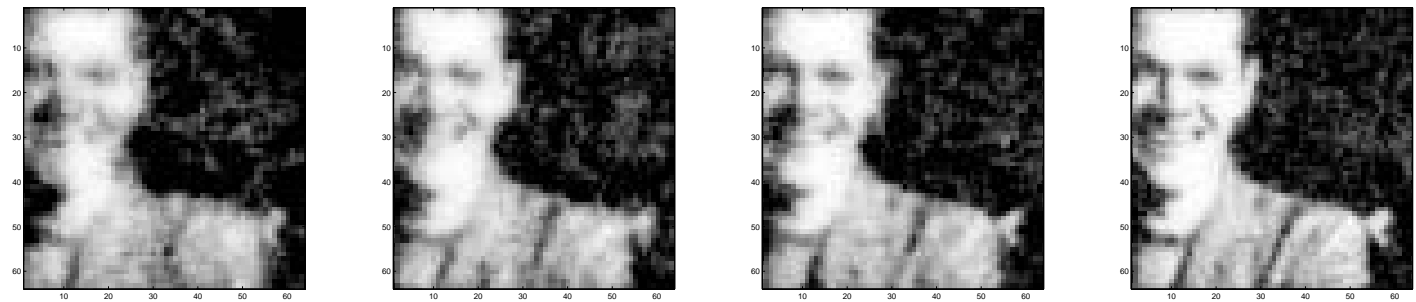

Figure 4.14: Constrained diffusion: steady state for $\lambda=0.5, \lambda=0.75, \lambda=1$ and $\lambda=2$ respectively with $\sigma=10 \%$

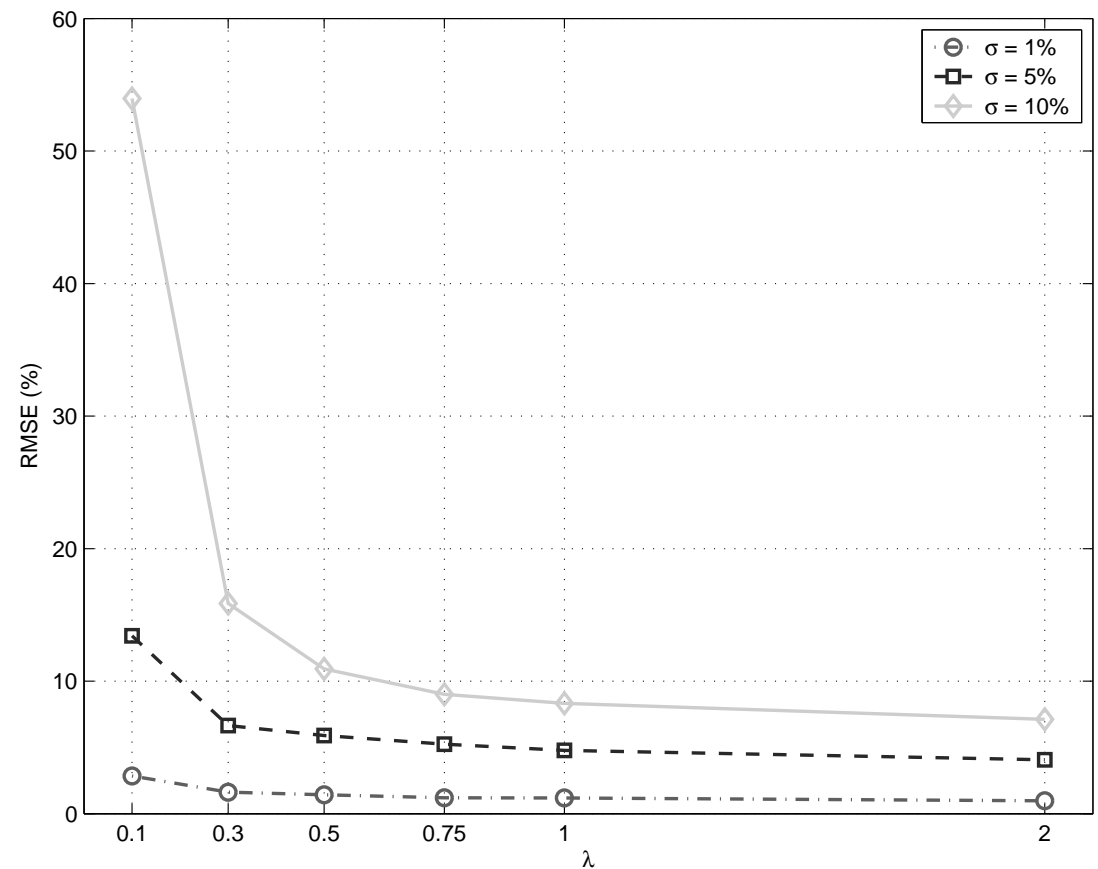

FiguRE 4.15: RMSE with respect to the ideal constrained diffusion 

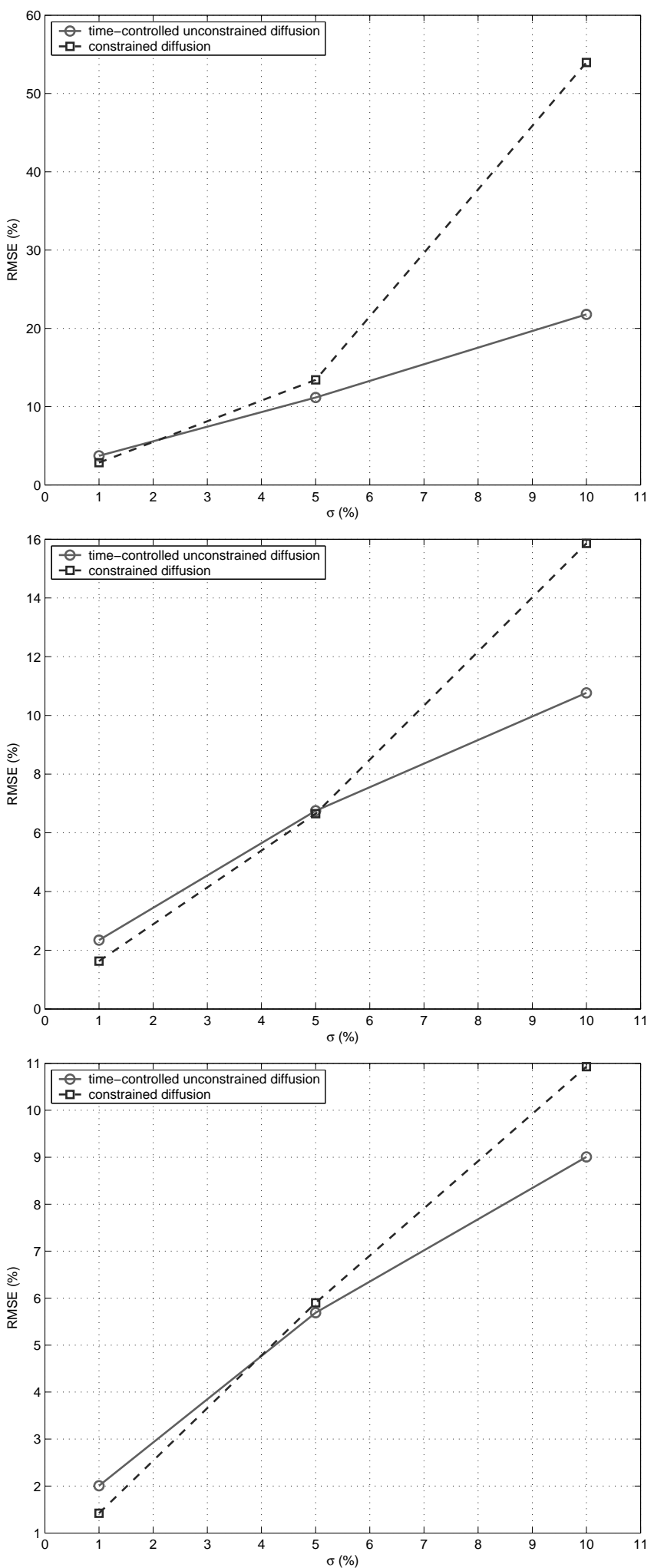

Figure 4.16: RMSE with respect to the ideal case for $\lambda=0.1 \Leftrightarrow t_{e q}=6.997 \tau, \lambda=0.3 \Leftrightarrow$ $t_{e q}=2.229 \tau$ and $\lambda=0.5 \Leftrightarrow t_{e q}=1.327 \tau$ respectively 

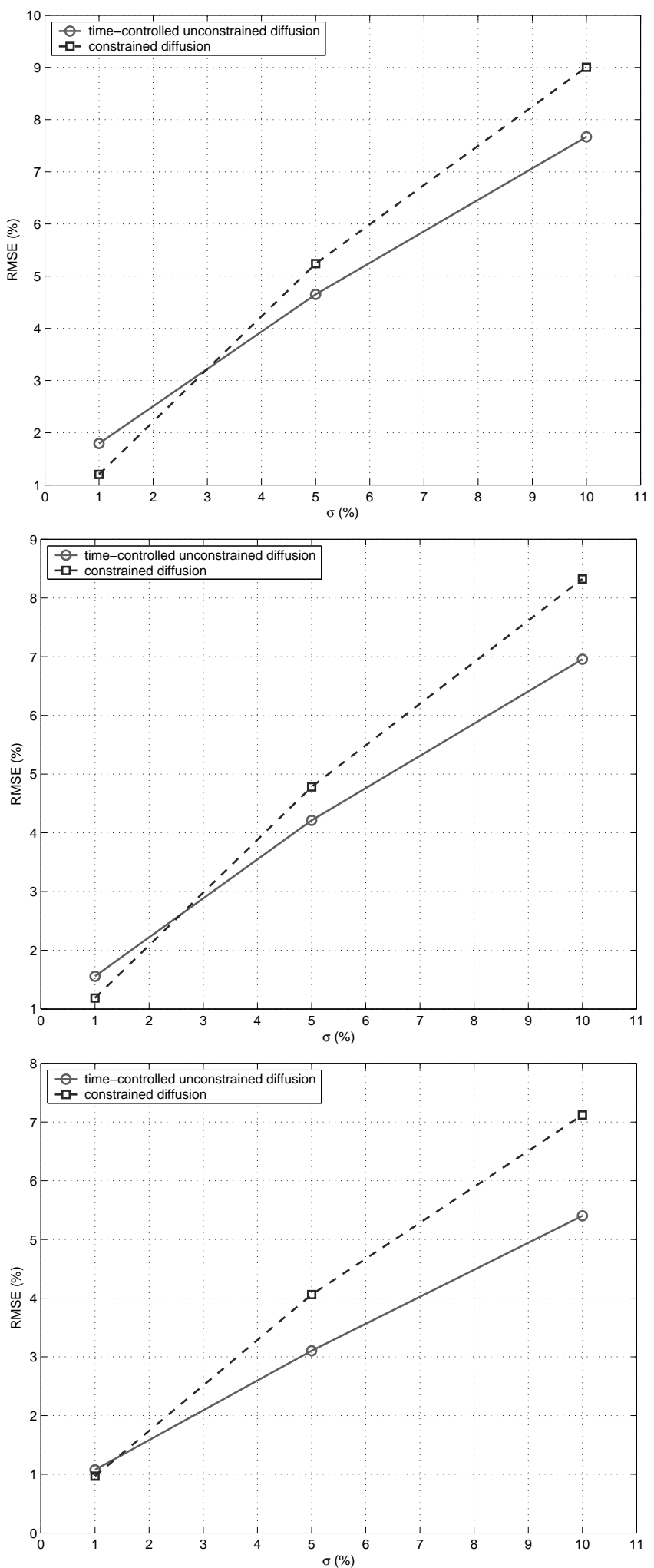

FIGURE 4.17: RMSE with respect to the ideal case for $\lambda=0.75 \Leftrightarrow t_{e q}=0.877 \tau, \lambda=1 \Leftrightarrow$ $t_{e q}=0.665 \tau$ and $\lambda=2 \Leftrightarrow t_{e q}=0.362 \tau$ respectively 


\begin{tabular}{|c|c|c|}
\hline$\lambda$ & $\begin{array}{c}\text { Constrained diffusion } \\
\text { Time to reach steady-state }\left(3 \tau^{\prime}\right) \\
\end{array}$ & $\begin{array}{l}\text { Time-controlled unconstrained diffusion } \\
\qquad t_{e q} \pm 0.01 \tau / 2 \\
\end{array}$ \\
\hline 2 & $1.5 \tau$ & $0.362 \tau$ \\
\hline 1 & $3 \tau$ & $0.665 \tau$ \\
\hline 0.75 & $4 \tau$ & $0.877 \tau$ \\
\hline 0.5 & $6 \tau$ & $1.327 \tau$ \\
\hline 0.3 & $10 \tau$ & $2.229 \tau$ \\
\hline 0.1 & $30 \tau$ & $6.997 \tau$ \\
\hline
\end{tabular}

TABLE 4.1: Equivalences between constrained and time-controlled unconstrained linear diffusion

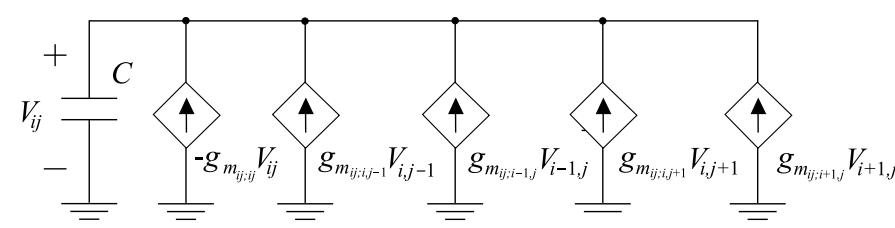

(a)

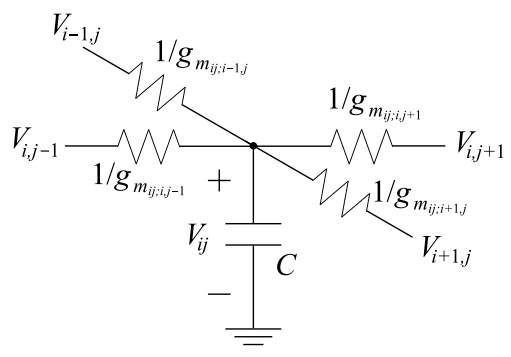

(b)

FiguRE 4.18: Node for unconstrained diffusion in (a) a transconductor-based CNN implementation and (b) an RC network.

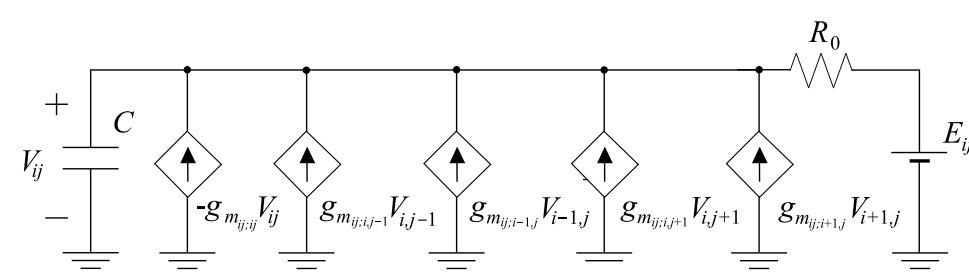

(a)

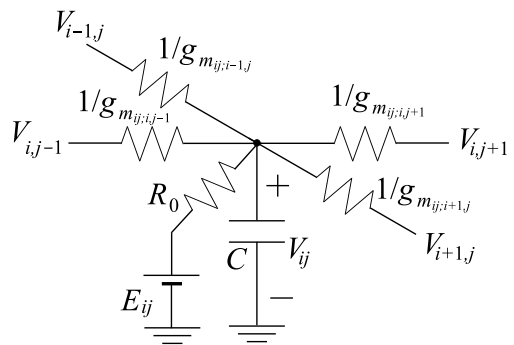

(b)

Figure 4.19: Node for constrained diffusion in (a) a transconductor-based CNN implementation and (b) a resistive grid.

However, when strict time requirements are mandatory, a CNN carrying out unconstrained linear diffusion reaches the desired radius of diffusion faster than the same CNN implementing constrained linear diffusion. According to the property expressed by Eq. (4.25), the slowest exponential controlling the ideal constrained diffusion dynamics until reaching steady-state presents a time constant $\tau^{\prime}=\tau / \lambda$. Considering that this exponential practically vanishes at $3 \tau^{\prime}$, we obtain the time instants at which ideal constrained diffusion reaches approximately steady-state, given in the second column of Table 4.1. As can be seen, $t_{e q}$ is always less than $3 \tau^{\prime}$, that is, time-controlled unconstrained linear diffusion is faster than constrained diffusion. 


\subsubsection{Physical origin for the non-convergence of the networks}

First of all, let us consider the case of the resulting $\mathrm{RC}$ network when $\lambda=0$, as depicted in Fig. 4.10(b). The evolution of the node voltages is described by:

$$
\left[\begin{array}{c}
\dot{V}_{1} \\
\dot{V}_{2}
\end{array}\right]=\frac{1}{R_{1} C_{p}}\left[\begin{array}{rr}
-1 & 1 \\
1 & -1
\end{array}\right]\left[\begin{array}{c}
V_{1} \\
V_{2}
\end{array}\right]
$$

Making $\tau=R_{1} C_{p}$, this equation is identical to Eq. (4.9). Again, if the network is initiated at $E_{1}$ and $E_{2}$, the equilibrium is reached at $V_{1}(\infty)=V_{2}(\infty)=\left(E_{1}+E_{2}\right) / 2$ as before. Now, the interesting aspect here is that, being supported by one single resistor, the dynamics of the network is not affected in essence by changes in the value of the resistor, which will only vary the time constant. When a larger resistive grid is considered, deviations have an influence on the final state of the pixels, but do not introduce a change in the dynamics of the network, i. e. there will always be one eigenvalue that equals zero and the rest will be negative. This result makes perfect sense as an RC network is composed of passive elements, i. e. elements that, like the resistor or the capacitor, do not extract energy from a bias network and inject it into the state variables.

In order to disclose the physical reason behind non-convergence in real transconductance-based CNNs implementing linear diffusion dynamics, let us compare the circuits in Fig. 4.18. The schematic in (a) corresponds to a node of a transconductance-based CNN implementing unconstrained linear diffusion. In order to cover all the possible dissimilarities between the elements that constitute the circuit, we consider 5 transconductances, generically $g_{m_{i j ; k l}}$, that weight the contribution of the state of cell $(k, l)$ to the evolution of cell $(i, j)$. In Fig. 4.18(b), we can see the schematic of an RC network node intended for unconstrained linear diffusion. The values of the 4 resistors are equated to the inverse of the transconductances in schematic (a) for the sake of comparison. Thus, the circuit in Fig. 4.18(b), which is inherently convergent, follows this equation:

$$
\begin{array}{r}
C \frac{d V_{i j}}{d t}=-\left(g_{m_{i j ; i, j-1}}+g_{m_{i j ; i-1, j}}+g_{m_{i j ; i, j+1}}+g_{m_{i j ; i+1, j}}\right) V_{i j}+g_{m_{i j ; i, j-1}} V_{i, j-1}+ \\
+g_{m_{i j ; i-1, j}} V_{i-1, j}+g_{m_{i j ; i, j+1}} V_{i, j+1}+g_{m_{i j ; i+1, j}} V_{i+1, j}
\end{array}
$$

While the circuit in Fig. 4.18(a) follows:

$$
C \frac{d V_{i j}}{d t}=-g_{m_{i j ; i j}} V_{i j}+g_{m_{i j ; i, j-1}} V_{i, j-1}+g_{m_{i j ; i-1, j}} V_{i-1, j}+g_{m_{i j ; i, j+1}} V_{i, j+1}+g_{m_{i j ; i+1, j}} V_{i+1, j}
$$




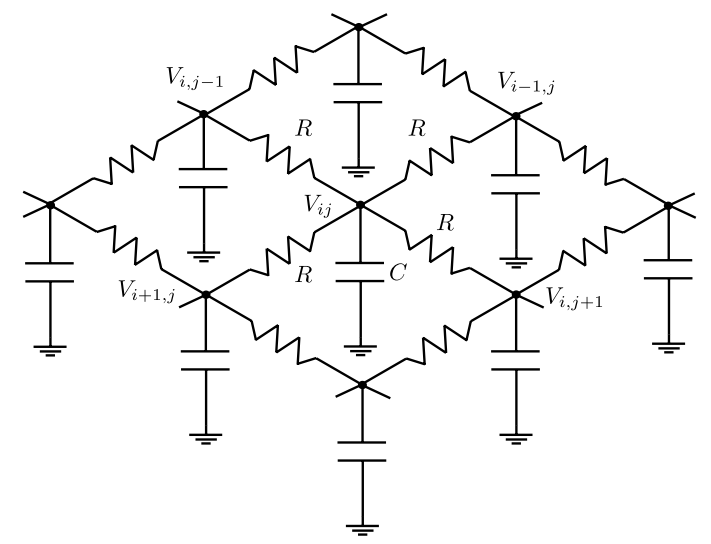

(a)

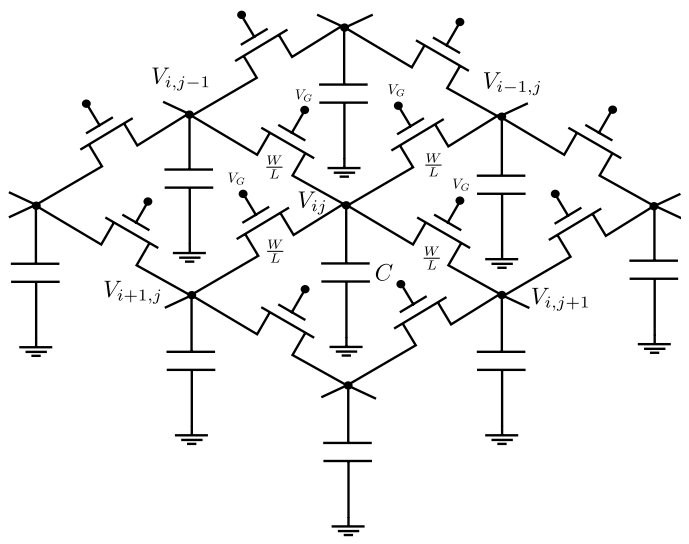

(b)

FiguRE 4.20: RC network performing linear diffusion (a) and its MOS-based counterpart (b)

that corresponds term by term to Eq. (4.34) if:

$$
g_{m_{i j ; i j}} \equiv g_{m_{i j ; i, j-1}}+g_{m_{i j ; i-1, j}}+g_{m_{i j ; i, j+1}}+g_{m_{i j ; i+1, j}}
$$

Notice that in the schematic in Fig. 4.18(b), the terms $g_{m_{i j ; k l}} V_{k l}$ and $-g_{m_{i j ; k l}} V_{i j}$ are introduced by the same element, i. e. the resistor labeled $1 / g_{m_{i j ; k l}}$. At the same time, in Fig. 4.18(a), these terms are implemented by different elements. One by the transconductance $g_{m_{i j ; k l}}$ and the other is aggregated into $g_{m_{i j ; i j}}$. This splitting of the current paths is subject to unbalances in any physical realization modeled by the circuit in Fig. 4.18(a).

Concerning constrained linear diffusion, Fig. 4.19(a), the inclusion of external signal sources does not eliminate the problem of unbalancing current paths with respect to its resistive grid counterpart, Fig. 4.19(b). Therefore, splitting these current contributions leads to unbalances in a physical implementation, which is the origin of non-convergence in transconductance-based CNN hardware implementing linear diffusion.

\subsection{Linear diffusion in time-controlled RC networks ${ }^{3}$}

Let us now focus on the resistive grid depicted in Fig. 4.9. It features two very good properties for the VLSI implementation of Gaussian filtering: robustness to mismatch and programmable width. However, it also demands variable resistors to set the filter width as well as variable signal sources over which each image composing a sequence can be mapped. A simpler way of attaining Gaussian filtering is through the RC network resulting from making $\lambda=0$ in this resistive grid (Fig. 4.20(a)). A real diffusion process takes place now within the network. An uneven charge

\footnotetext{
3 (C)2011 Wiley. Reprinted, with permission, from J. Fernández-Berni, R. Carmona-Galán, "All-MOS Implementation of RC Networks for Time-Controlled Gaussian Spatial Filtering". International Journal of Circuit Theory and Applications (published on-line on Feb. 23, 2011).
} 


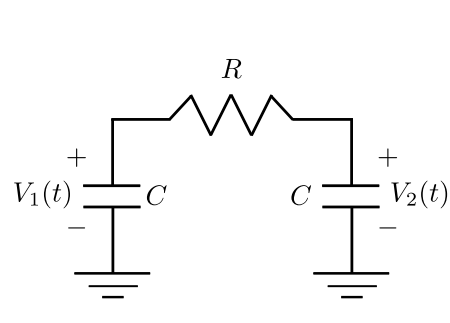

(a)

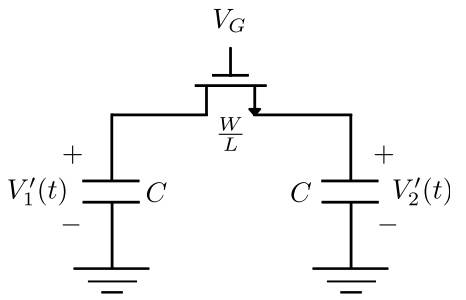

(b)

Figure 4.21: 2-node ideal RC network (a) and its MOS-based implementation (b)

distribution at the capacitors is diffused across the network and along time with a pace which is determined by the time constant $\tau=R C$. Eventually, a steady-state is reached when the charge is evenly distributed. The equation governing this dynamics is, precisely:

$$
\tau \frac{d V_{i j}}{d t}=-4 V_{i j}+V_{i+1, j}+V_{i-1, j}+V_{i, j+1}+V_{i, j-1}
$$

which, by making again $\tau=D^{-1}$, coincides exactly with Eq. (3.10), that is, the discrete-space diffusion equation.

Note that an RC network like that of Fig. 4.20(a) eliminates the problem of implementing variable resistors and variable signal sources. The value of the pixels can be easily mapped to the initial conditions of the capacitors and, without any additional energy contribution, the network will carry out their linear diffusion. However, other problems arise. First of all, it is necessary to stop the dynamics of the network at user-defined time instants in order to obtain targeted Gaussian filters, as described in Section 3.1. Secondly, the low sheet resistance exhibited by the most resistive materials available in standard CMOS requires very large areas for the necessary values of resistance.

Next, we demonstrate that these drawbacks can be overcome by employing MOS transistors biased in the ohmic region instead of resistors. MOS transistors permit to achieve larger resistances with much less area than resistors made with polysilicon or diffusion strips ${ }^{4}$. Additionally, the control of their gate voltage enables the control of the dynamics of the network. Our objective is therefore to design the MOS-based RC network in Fig. 4.20(b) in such a way that minimum error is committed when emulating its ideal counterpart in Fig. 4.20(a).

\subsubsection{Analysis of a 2-node network}

Let us compare the 2-node grids in Fig. 4.21 as a first approximation to the design of MOS resistors for RC networks. For purposes of clarity, we will confine the analysis to n-channel

\footnotetext{
${ }^{4}$ For example, in a standard $0.35 \mu \mathrm{m}$ CMOS process, a PMOS transistor with dimensions $0.4 / 1$ delivers an equivalent resistance of $85 \mathrm{k} \Omega$. To reach the same resistance with p-type diffusion, a strip of around 600 squares would be necessary.
} 
MOS transistors, but it applies equally well for p-type transistors. The gate voltage $V_{G}$ is fixed and we will assume, without loss of generality, that the initial conditions of the capacitors fulfill $V_{10}>V_{20}$, being $V_{10}=V_{10}^{\prime}$ and $V_{20}=V_{20}^{\prime}$. We will also assume that the transistor is biased in the triode region for any voltage at the drain and source terminals, that will range from $V_{\min }$ to $V_{\text {max }}$. The evolution of the circuit in Fig. 4.21(a) is described by this set of ODEs:

$$
\left\{\begin{array}{l}
C \frac{d V_{1}}{d t}=-\frac{V_{1}(t)-V_{2}(t)}{R} \\
C \frac{d V_{2}}{d t}=\frac{V_{1}(t)-V_{2}(t)}{R}
\end{array}\right.
$$

while the behavior of the circuit in Fig. 4.21(b) is described by:

$$
\left\{\begin{array}{rlr}
C \frac{d V_{1}^{\prime}}{d t} & =-\frac{V_{1}^{\prime}(t)-V_{2}^{\prime}(t)}{R_{M}(t)} \\
C \frac{d V_{2}^{\prime}}{d t} & =\frac{V_{1}^{\prime}(t)-V_{2}^{\prime}(t)}{R_{M}(t)}
\end{array}\right.
$$

being:

$$
R_{M}(t)=\frac{1}{k_{n} S_{n}\left\{V_{C}-\left[V_{1}^{\prime}(t)+V_{2}^{\prime}(t)\right]\right\}}
$$

where $k_{n}=\mu_{n} C_{o x}^{\prime} / 2, V_{C}=2\left(V_{G}-V_{T_{n}}\right)$ and $S_{n}=W / L$. Several key aspects must be clarified at this point about Eq. (4.40). Firstly, it corresponds to the instantaneous resistance of the transistor derived from the classical first-order approximation for the drain current of a NMOS biased in the triode region. This is really a coarse approximation for the real behavior of the transistors. However, it will permit to draw conclusions while keeping the equations reasonably manageable. These conclusions are confirmed not only by simulation, where the models include a great deal of second-order effects, but also in the physical implementation realized. It means that the mentioned first-order approximation summarizes the essential features of the transistors for a trustful design of MOS-based RC networks.

Secondly, due to charge conservation, Eq. (4.40) can be expressed as:

$$
R_{M}=\frac{1}{k_{n} S_{n}\left[V_{C}-\left(V_{10}^{\prime}+V_{20}^{\prime}\right)\right]}
$$

which means that, neglecting second-order effects, the resistance of the transistor depends on the sum of the initial conditions and does not vary along the corresponding diffusion. In other words, Eq. (4.41) is telling us that, if we choose a certain set of initial conditions within the prescribed signal range whose sum coincides and make $R_{M}=R$ for this sum, the dynamics for any initial conditions within this set will be perfectly emulated by the MOS network. On the contrary, there will always be an error for any other initial conditions outside the set as the resistance of the transistor during the diffusion will never match $R$. The question arising here is therefore: what is the value of the sum of initial conditions which, fulfilling $R_{M}=R$, 
minimizes the maximum error committed for any other possible value of this sum? That is, defining $V_{S}=V_{10}^{\prime}+V_{20}^{\prime}=V_{10}+V_{20}$, what is its optimum value $V_{S_{o p t}}$ for which making:

$$
\frac{1}{k_{n} S_{n}\left(V_{C}-V_{S_{o p t}}\right)}=R
$$

the maximum error committed by the MOS network for any other possible value of $V_{S}$ is minimum? The design equation of the MOSFET is immediately derived from Eq. (4.42):

$$
S_{n_{o p t}}=\frac{1}{k_{n} R\left(V_{C}-V_{S_{o p t}}\right)}
$$

where the value of $V_{S_{\text {opt }}}$ must be within the interval $\left[2 V_{\min }, 2 V_{\max }\right]$ according to the signal range previously established. Note that this design equation demands to know the exact value of $k_{n}$, which can present significant variations across the design space delimited by the corners of the process. We will see in the next chapter how to solve this problem in a real implementation.

In order to determine $V_{S_{o p t}}$, notice firstly that the charge extracted from one capacitor will end up in the other at both the ideal network and the MOS-based network. We can thus define the error in the corresponding node voltages as:

$$
\left\{\begin{array}{r}
V_{1}^{\prime}(t)=V_{1}(t)+\epsilon(t) \\
V_{2}^{\prime}(t)=V_{2}(t)-\epsilon(t)
\end{array}\right.
$$

or, equivalently:

$$
\epsilon(t)=\frac{V_{1}^{\prime}(t)-V_{2}^{\prime}(t)}{2}-\frac{V_{1}(t)-V_{2}(t)}{2}
$$

Since our initial assumptions were $V_{10}=V_{10}^{\prime}$ and $V_{20}=V_{20}^{\prime}$, we have that $\epsilon(0)=0$. Also, the stationary state, reached when $t \rightarrow \infty$, renders $\epsilon(\infty)=0$, as $V_{1}(\infty)=V_{2}(\infty)$ and $V_{1}^{\prime}(\infty)=$ $V_{2}^{\prime}(\infty)$. Therefore, there must be at least one point in time, let us call it $t_{e x t}$, in which the error reaches an extreme value, either positive or negative. In order to find this time instant, time derivative is firstly applied to Eq. (4.45), obtaining:

$$
\frac{d \epsilon}{d t}=\frac{1}{2} \frac{d}{d t}\left[V_{1}^{\prime}(t)-V_{2}^{\prime}(t)\right]-\frac{1}{2} \frac{d}{d t}\left[V_{1}(t)-V_{2}(t)\right]
$$

Taking into account now that, from Eq. (4.39):

$$
\frac{d}{d t}\left[V_{1}^{\prime}(t)-V_{2}^{\prime}(t)\right]=-2 \frac{V_{1}^{\prime}(t)-V_{2}^{\prime}(t)}{R_{M} C}
$$

and from Eq. (4.38): 


$$
\frac{d}{d t}\left[V_{1}(t)-V_{2}(t)\right]=-2 \frac{V_{1}(t)-V_{2}(t)}{R C}
$$

we can rewrite Eq. (4.46) as:

$$
\frac{d \epsilon}{d t}=\frac{V_{1}(t)-V_{2}(t)}{R C}-\frac{V_{1}^{\prime}(t)-V_{2}^{\prime}(t)}{R_{M} C}
$$

where, taking into account that $\tau=R C$, we have:

$$
\tau \frac{d \epsilon}{d t}=V_{1}(t)-V_{2}(t)-\frac{R}{R_{M}}\left[V_{1}^{\prime}(t)-V_{2}^{\prime}(t)\right]
$$

Finally, considering that, according to Eq. (4.44):

$$
V_{1}(t)-V_{2}(t)=V_{1}^{\prime}(t)-V_{2}^{\prime}(t)-2 \epsilon(t)
$$

and substituting Eq. (4.41) and Eq. (4.42) in Eq. (4.50), we obtain:

$$
\tau \frac{d \epsilon}{d t}=\left[V_{1}^{\prime}(t)-V_{2}^{\prime}(t)\right] \frac{V_{10}+V_{20}-V_{S_{o p t}}}{V_{C}-V_{S_{o p t}}}-2 \epsilon(t)
$$

where we have also applied our initial assumption regarding the initial conditions, that is, $V_{10}=$ $V_{10}^{\prime}$ and $V_{20}=V_{20}^{\prime}$. This time derivative of the error must cancel in $t_{\text {ext }}$, resulting in an extreme error of:

$$
\epsilon_{e x t}=\frac{1}{2}\left[V_{1}^{\prime}\left(t_{e x t}\right)-V_{2}^{\prime}\left(t_{e x t}\right)\right] \frac{V_{10}+V_{20}-V_{S_{o p t}}}{V_{C}-V_{S_{o p t}}}
$$

where $V_{C}$ is a constant, $V_{S_{\text {opt }}}$ is a design parameter and $V_{1}^{\prime}\left(t_{e x t}\right)$ and $V_{2}^{\prime}\left(t_{e x t}\right)$ are variables which can be referred to the initial conditions by solving Eq. (4.39), obtaining:

$$
\epsilon_{e x t}=\frac{1}{2}\left(V_{10}-V_{20}\right) \frac{V_{10}+V_{20}-V_{S_{o p t}}}{V_{C}-V_{S_{o p t}}} e^{\frac{-2 t_{e x t}}{R_{M} C}}
$$

Finally, $t_{\text {ext }}$ can be found by solving Eq. (4.38) and Eq. (4.39) and making use of Eq. (4.44):

$$
t_{e x t}=\frac{\tau}{2} \frac{\ln (r)}{r-1}
$$

where:

$$
r=\frac{R}{R_{M}}=\frac{V_{C}-V_{10}-V_{20}}{V_{C}-V_{S_{o p t}}}
$$

Substituting Eq. (4.55) in Eq. (4.54), we have the following expression: 


$$
\epsilon_{\text {ext }}=\frac{1}{2}\left(V_{10}-V_{20}\right) \frac{V_{10}+V_{20}-V_{S_{o p t}}}{V_{C}-V_{S_{o p t}}} r^{\frac{r}{1-r}}
$$

from which the first important conclusion can be extracted. The extreme error is independent of $S_{n}$ and $k_{n}$. That is to say, once $S_{n}$ is defined by Eq. (4.43) for a value of $k_{n}$ known, the error committed by the MOS network does not depend on these parameters and therefore is not affected by their mismatch. This robustness to mismatch is confirmed both by simulation and in the physical implementation presented in this book.

Unfortunately, the exact analytical expression for the extremes of $\epsilon_{\text {ext }}$ from Eq. (4.57) can not be obtained. This in turn implies that the exact analytical expression for $V_{S_{o p t}}$ can not be found either. However, a good approximation for them is still possible. Let us take a look to Eq. (4.56). The value of $r$ depends on a quotient where $V_{C}$ is the dominant term at both the numerator and the denominator for deep triode biasing. Let us therefore assume that $\partial r / \partial V_{10} \simeq$ 0 and $\partial r / \partial V_{20} \simeq 0$. Under these conditions, it can be demonstrated that only a critical point, more precisely a saddle point, can be found at $\left(V_{S_{o p t}} / 2, V_{S_{o p t}} / 2\right)$. Therefore, we can only talk of absolute maxima or minima which will be at the boundaries of the domain considered for $\left(V_{10}, V_{20}\right)$. The initial assumption $V_{10}>V_{20}$ reduces the boundaries to be searched to only two. The first is that one set by making $V_{10}=V_{\max }$, that is, according to Eq. 4.57:

$$
\left.\epsilon_{e x t}\right|_{V_{10}=V_{\max }}=\frac{1}{2}\left(V_{\max }-V_{20}\right) \frac{V_{\max }+V_{20}-V_{S_{o p t}}}{V_{C}-V_{S_{o p t}}} r^{\frac{r}{1-r}}
$$

from which calculating the derivative with respect to $V_{20}$ and bearing in mind our assumption that $\partial r / \partial V_{20} \simeq 0$, we have:

$$
\left.\frac{d}{d V_{20}} \epsilon_{e x t}\right|_{V_{10}=V_{\max }}=\frac{1}{2} \frac{V_{S_{o p t}}-2 V_{20}}{V_{C}-V_{S_{o p t}}} r^{\frac{r}{1-r}}
$$

and finally, by making this expression equal to 0, the location of the first absolute extreme is obtained, $\left(V_{10}, V_{20}\right)=\left(V_{\max }, V_{S_{\text {opt }}} / 2\right)$. The other boundary to search absolute extremes is that one set by making $V_{20}=V_{\min }$. It means, according to Eq. 4.57, that:

$$
\left.\epsilon_{\text {ext }}\right|_{V_{20}=V_{\text {min }}}=\frac{1}{2}\left(V_{10}-V_{\text {min }}\right) \frac{V_{10}+V_{\text {min }}-V_{S_{o p t}}}{V_{C}-V_{S_{o p t}}} r^{\frac{r}{1-r}}
$$

We calculate now the derivative with respect to $V_{10}$, assuming $\partial r / \partial V_{10} \simeq 0$ :

$$
\left.\frac{d}{d V_{10}} \epsilon_{e x t}\right|_{V_{20}=V_{\min }}=\frac{1}{2} \frac{2 V_{10}-V_{S_{o p t}}}{V_{C}-V_{S_{o p t}}} r^{\frac{r}{1-r}}
$$


and again, by making this expression equal to 0 , the second absolute extreme is located at point $\left(V_{10}, V_{20}\right)=\left(V_{S_{\text {opt }}} / 2, V_{\text {min }}\right)$. Finally, by substituting these two points in Eq. 4.57 , the absolute extremes are respectively obtained:

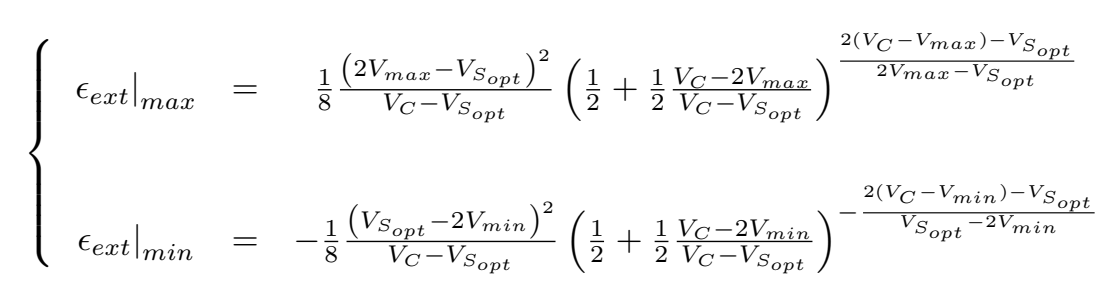

The final step is to determine the value of $V_{S_{o p t}}$ which minimizes $\max \left|\epsilon_{\text {ext }}\right|$. To this end, let us again assume deep triode biasing. In this way, the exponential terms in $\left.\epsilon_{e x t}\right|_{\text {max }}$ and in $\left.\epsilon_{\text {ext }}\right|_{\min }$ can be approximated by 1 when varying $V_{S_{\text {opt }}}$ within the range of its possible values $\left[2 V_{\min }, 2 V_{\max }\right]$. Applying this approximation, it can be seen that to increase or to decrease $V_{S_{\text {opt }}}$ has antagonistic effects in the magnitude of $\left.\epsilon_{e x t}\right|_{\text {max }}$ and $\left.\epsilon_{e x t}\right|_{\text {min }}$, being:

$$
V_{S_{o p t}}=V_{\min }+V_{\max }
$$

the expression for $V_{S_{o p t}}$ which minimizes the magnitude of the error:

$$
\min \left(\max \left|\epsilon_{e x t}\right|\right)=\frac{1}{8} \frac{\left(V_{\max }-V_{\min }\right)^{2}}{V_{C}-V_{\min }-V_{\max }}
$$

Notice that this minimized error depends inversely on $V_{C}$, that is, on $V_{G}-V_{T_{n}}$, which was considered fixed at the beginning of the design process. Thus, $V_{G}$ and $V_{T_{n}}$ must be chosen in such a way that makes their difference as large as possible.

Finally, by substituting Eq. (4.63) in Eq. (4.43), the design equation for minimum error is obtained:

$$
S_{n_{o p t}}=\frac{1}{k_{n} R\left(V_{C}-V_{\min }-V_{\max }\right)}
$$

This conclusion about $V_{S_{o p t}}$ invalidates the groundless intuition that the optimal design could be derived from equaling the midpoint of the interval of possible values of $R_{M}$ to $R$. On the contrary, the value of $R_{M}$ which matches $R$ for optimal design is notably below such a midpoint.

It is time now to numerically corroborate the validity of the assumptions realized and, on the way, to know the magnitude of the error achieved by the optimum design. In Table 4.2 some results are showed for typical values of the voltages involved. As above mentioned, the larger $V_{G}$ the smaller the error. Thus, two usual maximum biasing voltages for the transistor gate in current CMOS technologies are introduced in Table 4.2. Regarding $V_{T_{n}}$, two typical related values are used. We do not take into account the possibility of using low- or even zero-threshold 
transistors currently offered by some manufacturers, what would further reduce the error. Note that the columns labeled as max $e_{e x t}, \min e_{e x t}$ and $V_{S_{\text {opt }} \text { um }}$ are numerically calculated from Eq. (4.57) whereas those ones labeled as $e_{\max }, e_{\min }$ and $V_{S_{o p t}}$ are directly calculated from Eq. (4.62) and Eq. (4.63) respectively. Several conclusions can be drawn from a careful analysis of Table 4.2. First of all, the deeper the ohmic biasing the less the error committed by the MOS network and the better the approximations applied, as expected. Secondly, despite the large signal swings considered and therefore the large variation of the instantaneous resistance of the MOSFET, represented by the wide interval of possible values of $r$, the optimal design keeps the error moderately small. It is due to the suitability of this design for the diffusion dynamics. Note that in the ideal network, the maximum charge injection occurs when one of the nodes equals $V_{\max }$ whereas the other equals $V_{\min }$. This situation can only exist just at the beginning of the diffusion. A significant error committed by the MOS-based grid at this point would mean to noticeably alter the rest of the dynamics. However, such a configuration of the voltages makes their sum equal to $V_{\min }+V_{\max }$ and therefore the MOS network does not commit any error at all. On the contrary, the error committed by the MOS grid is maximum when the voltages of the nodes involved coincide at $V_{\min }$ or $V_{\max }$, being their sum $2 V_{\min }$ or $2 V_{\max }$ respectively. But there is no charge injection between the nodes in these cases as their voltages are the same. Therefore, the maximum error is committed when the dynamics is not affected. Finally, remark that no specific value of $R$ has been included in Table 4.2 as it is not necessary to compute the error. Once set $V_{G}, V_{T_{n}}$ and $\left[V_{\min }, V_{\max }\right], S_{n_{\text {opt }}}$ can ideally take, from Eq. (4.65), any value according to the targeted $R$ without affecting the magnitude of the error.

\subsubsection{Networks of arbitrary size}

Taking into account the mathematical framework deployed to optimize the design of a 2-node MOS network, it is obvious that the extension of the results to networks of arbitrary size can not be addressed in the same way. Our proposal for such extension is a stochastic approach. Let us suppose a $M \times N$ RC grid similar to that of Fig. 4.20(a) where every initial value of pixel can be modeled by a random variable with a uniform distribution between $V_{\min }$ and $V_{\max }$, that is, $\mathbf{V}_{\mathbf{i j}}(\mathbf{0}) \sim U\left(V_{\min }, V_{\max }\right)$, according to notation in [Papoulis \& Unnikrishna, 2002]. Such a distribution is depicted in Fig. 4.22(a). This is a rather reasonable supposition, specially if the grid is intended to process natural images. In this way, if we choose any two neighbor nodes of the network, namely $(i, j)$ and $(k, l)$, the resulting distribution of the sum of both nodes is a triangle like that one represented in Fig. 4.22(b). It can be seen that the most probable value of the sum is $V_{\min }+V_{\max }$. Let us see what happens at the end of the processing. Consider again Eq. (3.13), which defines the filtering carried out by an RC network along time. Notice that the dc component is the only one that is not affected by the filtering, that is, $\hat{G}_{00}(t)=1 \forall t$. It means that the average value of the pixels does not change during the processing. Thus, when 


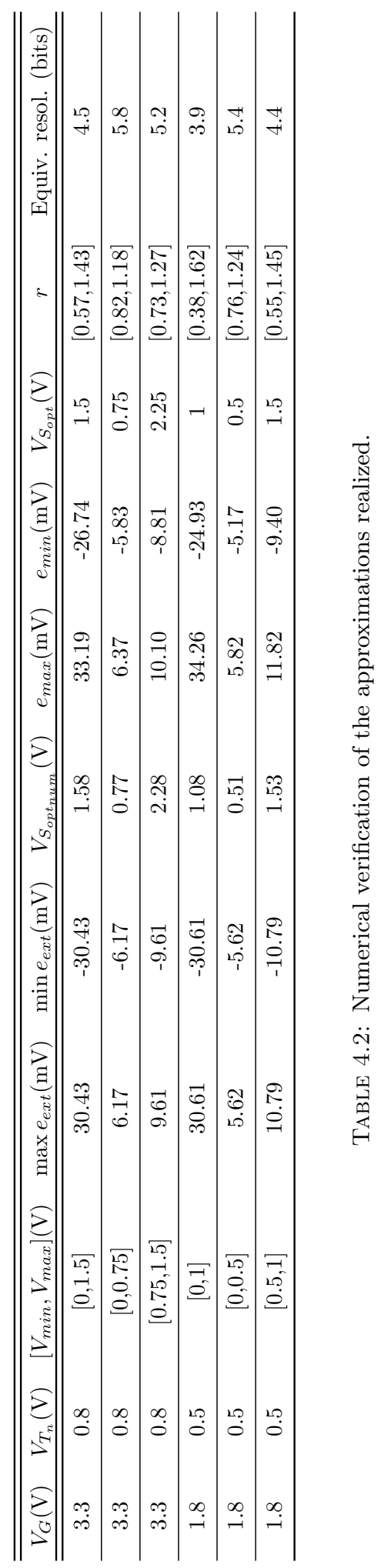




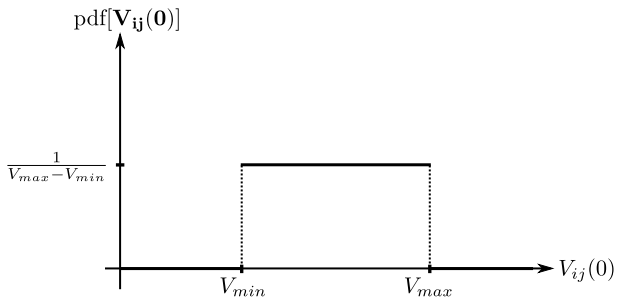

(a)

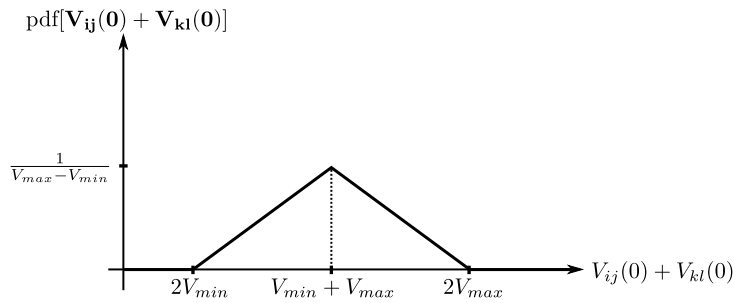

(b)

Figure 4.22: Probability density function for the initial voltage (a) and for the sum of any two initial voltages (b) at the nodes of a RC network.

the diffusion is running, the higher frequencies left are progressively filtered until all of them are eventually removed except the dc component. In other words, the values of the pixels are progressively getting closer until all of them eventually coincide at the mean value, which has never been altered. Let $\overline{\mathbf{V}}$ be the random variable representing the mean value of the pixels of an image. It can be expressed as:

$$
\overline{\mathbf{V}}=\frac{\mathbf{V}_{\mathbf{1}, \mathbf{1}}(\mathbf{0})+\mathbf{V}_{\mathbf{1 , 2}}(\mathbf{0})+\ldots+\mathbf{V}_{\mathbf{i j}}(\mathbf{0})+\ldots+\mathbf{V}_{\mathbf{M}, \mathbf{N}}(\mathbf{0})}{M N}
$$

from which, taking into account that every pixel $\mathbf{V}_{\mathbf{i j}}(\mathbf{0})$ presents an uniform distribution and by applying the central limit theorem, we can conclude that $\overline{\mathbf{V}}$ approaches a normal distribution $N\left(\mu, \sigma^{2}\right)$ as follows:

$$
\overline{\mathbf{V}} \sim N\left[\frac{V_{\min }+V_{\max }}{2}, \frac{\left(V_{\max }-V_{\min }\right)^{2}}{12}\right]
$$

and as every pixel will reach the mean value at the end of the diffusion process, $\mathbf{V}_{\mathbf{i j}}(\infty)$ presents the same distribution as defined by Eq. (4.67), depicted in Fig. 4.23(a). It implies that the most probable value of the sum of any two voltages of the network by the end of the diffusion is again $V_{\min }+V_{\max }$, as showed in Fig. 4.23(b). We have seen what happens at the beginning and at the end of the diffusion, but what about the processing itself? We start from uniform distributions for every pixel. When the diffusion is being performed, the probability of a certain pixel to reach the mean value is constantly increasing as the probability of having filtered all the frequencies other than the dc component also increases. Note that, according to Eq. (3.13), any time interval carrying out diffusion implies necessarily the filtering of frequencies other than the dc component. Thus, the uniform distribution represented in Fig. 4.22(a) is transformed along the diffusion until eventually becoming that in Fig. 4.23(a). However, the interest for us in this transformation falls on the fact that, on increasing the probability of a certain pixel to reach the mean value, its most probable value during the diffusion is $\left(V_{\min }+V_{\max }\right) / 2$ as this is the most probable value of the mean value. And it in turn means that the most probable value of the sum of any two voltages within the ideal network at any time instant during the 


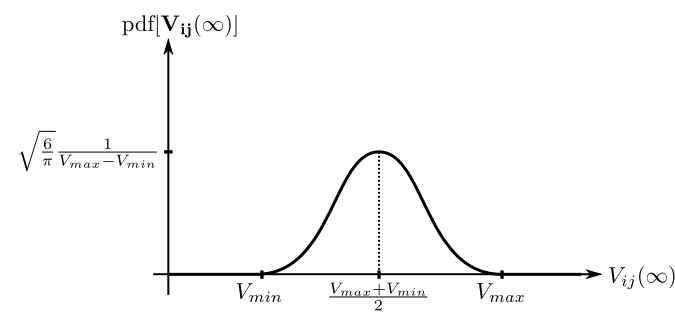

(a)

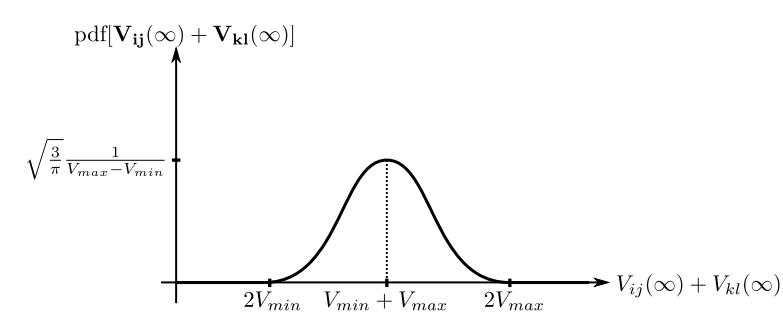

(b)

Figure 4.23: Probability density function for the final voltage (a) and for the sum of any two final voltages (b) at the nodes of a RC network.

diffusion is $V_{\min }+V_{\max }$. From this result, and keeping in mind that the elementary transistor at a MOS-based RC grid emulating the ideal network just described presents an instantaneous resistance:

$$
R_{M_{i j, k l}}(t)=\frac{1}{k_{n} S_{n}\left\{V_{C}-\left[V_{i j}^{\prime}(t)+V_{k l}^{\prime}(t)\right]\right\}}
$$

it can be concluded that this value of resistance should match the resistor of the ideal network for the most probable value of the sum of any two neighbor voltages, that is:

$$
\frac{1}{k_{n} S_{n}\left[V_{C}-\left(V_{\min }+V_{\max }\right)\right]}=R
$$

which directly leads to the design equation obtained for the 2-node case:

$$
S_{n_{\text {opt }}}=\frac{1}{k_{n} R\left(V_{C}-V_{\min }-V_{\max }\right)}
$$

In this way, we make sure that the instantaneous diffusion performed by each elementary MOS transistor between its drain and source terminals equals that of the corresponding ideal resistor for most of the time instants of the dynamics, introducing thus minimum error from a stochastic point of view. Note that we are implicitly assuming that this error is small enough to apply to the MOS grid the same considerations extracted from the ideal grid regarding the distributions and most probable values of the voltages during the diffusion. The simulation results presented in the next section confirm that this assumption can be made.

\subsubsection{Simulation of a $64 \times 64$ network}

This section addresses the design of a $64 \times 64$ MOS-based RC network. The objective is to confirm the validity of the guidelines drawn in the previous section despite having made use of a coarse approximation for the behavior of the transistors in the triode region. Simulations have been realized using standard $0.35 \mu \mathrm{m}$ CMOS $3.3 \mathrm{~V}$ process transistor models in HSPICE. The 


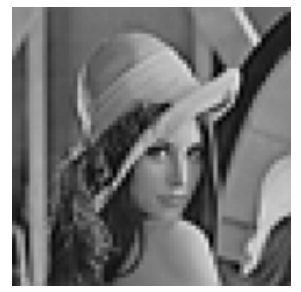

(a)

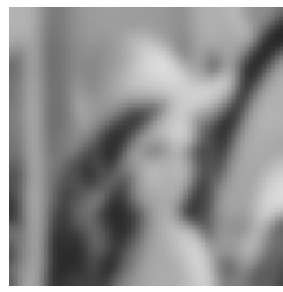

(b)

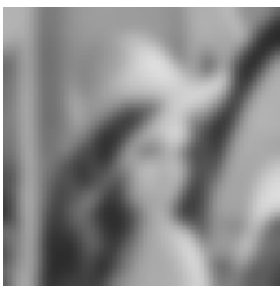

(c)

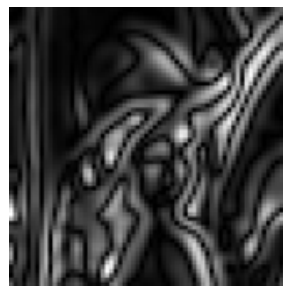

(d)

Figure 4.24: Comparison between a MOS-based RC network and its ideal counterpart: (a) Original image, (b) MOS-diffused image at the instant of maximum error, (c) image diffused by the corresponding ideal RC network, (d) absolute error normalized to maximum individual pixel error

signal range at the nodes is $[0 \mathrm{~V}, 1.5 \mathrm{~V}]$, wide enough to evidence the influence of the MOSFET nonlinearities over the Gaussian filtering performed by the grid. $V_{G}$ is established at $3.3 \mathrm{~V}$ in order to bias the transistor as deep in the ohmic region as possible. The design specification is to implement a RC network with $\tau=100 \mathrm{~ns}$ by using a resistor $R=100 \mathrm{k} \Omega$ and a capacitor $C=1 \mathrm{pF}$. The sizing of the elementary NMOS transistor to achieve this value of $R$ is based on Eq. (4.70). But this equation does not take into account second-order effects like for example the body effect. It means that $S_{n}$ does not only depend on the sum $V_{\min }+V_{\max }$ but also on the values of the voltages at drain and source that render that sum. Thus, for a specific value of $S_{n}$, the instantaneous resistance implemented by the transistor can vary $\pm 5 \%$ depending on the drain and source voltages applied. In order to take into account these variations, we have selected the minimum possible width $W=0.4 \mu \mathrm{m}$. Then, we have swept $L$ until finding that value which makes the average resistance of the transistor for all the possible voltages at the drain and source terminals rendering the optimum sum, i. e. $V_{\min }+V_{\max }$, equals to $100 \mathrm{k} \Omega$. The result is $L=7.54 \mu \mathrm{m}^{5}$.

Once designed the elementary transistor, let us suppose that the initial voltage at the capacitors is proportional to the image intensity displayed at Fig. 4.24(a). The MOS-based RC network runs the diffusion over these initial voltages in parallel with its ideal counterpart in order to be compared. The deviation is measured via the RMSE (Fig. 4.25(a)) and reaches a maximum soon after the beginning of the diffusion process. The state of the corresponding nodes in both networks at this point, displayed in Figs. 4.24(b) and 4.24(c), is perceptually equivalent. The maximum observed RMSE for the complete image is $0.5 \%$, while the maximum individual pixel error is $1.76 \%$. The RMSE remains below $0.6 \%$ - equivalent resolution between 6 and 7 bits even introducing an exaggerated mismatch (10\%) in the transistors' $V_{T_{n 0}}$ and $\mu_{n}$ (Fig. 4.25(b)), confirming thus the robustness to mismatch predicted in Section 4.3.1.

\footnotetext{
${ }^{5}$ This transistor length lies out of the physical design grid, that fixes the minimum feature size to be $0.05 \mu \mathrm{m}$. We are using it here as illustrative of the design procedure.
} 
(a)

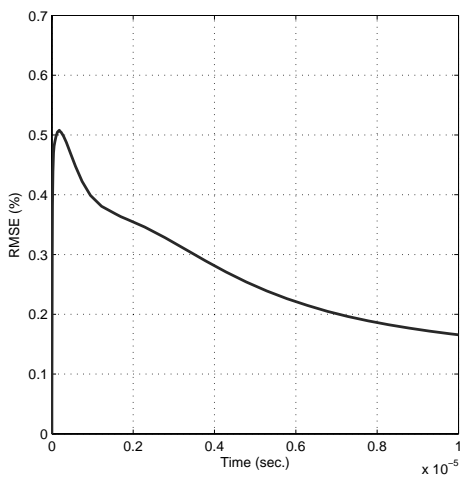

FiguRE 4.25: RMSE of the MOS-based grid state vs. ideal RC grid state: (a) w/o mismatch, (b) Monte Carlo with $10 \%$ mismatch

\subsection{Summary}

The basis for a robust VLSI implementation of linear diffusion has been established. The use of the CNN framework has been dismissed because of the sensitivity to mismatch of its typical hardware implementation based on transconductances. On the contrary, it is precisely the robustness to mismatch one of the most valuable characteristic of RC networks. We have demonstrated that an accurate VLSI implementation of these networks, supported by MOS resistors, is feasible. In addition to delivering high values of resistance, MOS resistors enable the control of the dynamics of the network through their gate voltage. In the following chapter, we describe a prototype vision chip which makes use successfully of the design guidelines for MOS-based RC networks just defined. This prototype also implements the rest of processing primitives studied in Chapter 3. 



\section{Chapter 5}

\section{FLIP-Q: A QCIF resolution}

\section{focal-plane array for low-power}

\section{image processing}

\subsection{Introduction ${ }^{1}$}

The vision processing chain is usually divided into three consecutive steps [González \& Woods, 2002]: i) low-level tasks, where both inputs and outputs are images, ii) medium-level tasks, where inputs are images but outputs are attributes extracted from inputs and iii) high-level tasks, which perform the cognitive functions associated to vision from the result of low- and medium-level tasks. The most remarkable features of low-level tasks are, as we saw in Chapter 2, their regularity and potential parallelism. All pixels are equally processed and the result of the processing over each pixel is usually independent from the result of the computations over the rest. These features are not exploited at all by conventional imager-memory-DSP architectures, which result quite inefficient when it comes to carry out low-level image processing. For these architectures, the speed of the digital processor must be high in order to handle the massive serialized data flow and the repeated memory accesses, what drastically affects the power consumption [Rabaey, 1995]. Alternative architectures can be proposed to handle lowlevel image processing tasks more efficiently. They can take advantage of the moderate accuracy usually required in early vision models [Poynton, 2007]. For example, instead of simply delivering the already captured raw data, a reduced representation of the scene can be elaborated with

\footnotetext{
${ }^{1}$ From here to section 5.6, (C)2011 IEEE. Reprinted, with permission, from J. Fernández-Berni, R. CarmonaGalán, L. Carranza-González, "FLIP-Q: A QCIF Resolution Focal-Plane Array for Low-Power Image Processing". IEEE Journal of Solid-State Circuits, Vol. 46, No. 3, pp. 669-680, March 2011.
} 
relatively coarse circuitry at the focal plane. Higher level vision tasks can be implemented then by conventional digital architectures, now operating on a reduced processing load, and consequently lowering the overall power consumption.

This architectural scheme has been incorporated to either general-purpose vision chips [Liñán et al., 2004; Dudek \& Hicks, 2005; Dubois et al., 2008; Poikonen et al., 2009] or to applicationspecific smart image sensors [Zhiqiang et al., 2008; Nilchi et al., 2009]. The basic idea behind these approaches is to incorporate processing circuitry next to the photosensor at every pixel, obtaining thus focal-plane processing arrays. In such arrays, the Single Instruction Multiple Data (SIMD) paradigm, sketched back in 1958 [Unger, 1958], is usually applied. All the PEs execute the same instructions while making computations on different data. Although SIMDbased focal-plane processing arrays composed of digital PEs have been proposed [Komuro et al., 2009; Chih-Chi et al., 2009], currently the analog implementations continue to be more areaand power-efficient than their digital counterparts.

This chapter reports a $176 \times 144$-px smart image sensor which implements a massively parallel SIMD-based focal-plane processing array composed of pixel-level PEs. These PEs, which carry out analog image processing concurrently with photosensing, can be grouped into fullyprogrammable rectangular-shape areas by loading the appropriate interconnection patterns into the registers at the edge of the array. The targeted processing can be thus performed block-wise. Readout is done pixel-by-pixel in a random access fashion. An 8b ADC is provided on-chip. The image processing primitives implemented by the chip, experimentally tested and fully functional, are those ones defined in Chapter 3. The power consumption associated to the capture, processing and A/D conversion of an image flow at 30fps, with full-frame processing but reduced frame size output, ranges from $2.7 \mathrm{~mW}$ to $5.6 \mathrm{~mW}$, depending on the operation to be performed. The chip has been designed and fabricated in the AMS 0.35 $\mu \mathrm{m}$ CMOS-OPTO process. This CMOS process does not incorporate any special device for image sensing. Indeed, it only differs from the standard AMS $0.35 \mu \mathrm{m}$ process in an anti-reflective coating and an EPI substrate which reduces the dark current. The chip contains around half million transistors, $98 \%$ of them working in analog mode.

\subsection{Architecture}

The architecture of the chip is depicted in Fig. 5.1. The analog core is a $176 \times 144$ array of PEs with concurrent photodiodes. The PEs are 4-connected. Each of these connections can be enabled or disabled column-wise and row-wise across the array. The focal plane can be thus divided into independent rectangular blocks whose size is defined by the user by selecting which columns and rows of PEs are interconnected. Note that the size of the blocks could vary across 
the focal plane. Once this block-based division is set, the control logic for diffusion and energy computation generates the corresponding signals to perform the processing primitives defined in Chapter 3.

The outcome of the processing can be read out pixel-wise by selecting the column and row where the desired pixel is located. The value of the pixel is buffered at the column bus and delivered to an $8 \mathrm{~b}$ SAR ADC, which finally outputs the digitalized result. Although the inclusion of only one ADC prevents the chip from reaching high frame rates as a full-resolution imager, it greatly reduces the power consumption while still allowing a remarkable throughput for the simplified representations of the scene achievable at the focal plane.

The main characteristics of the chip are summarized in Table 5.1. A general view of the prototype packaged is shown in Fig. 5.2 along with a microphotograph of the chip with a close-up of the photosensors.

\subsection{Image processing implementation}

The elementary cell of the analog core is depicted in Fig. 5.3, and a timing diagram with the control signals and the waveform of the voltage at the most relevant nodes of the basic cell is shown in Fig. 5.4. The nominal reset voltage of the photodiode and the sensing capacitance $C_{P}$

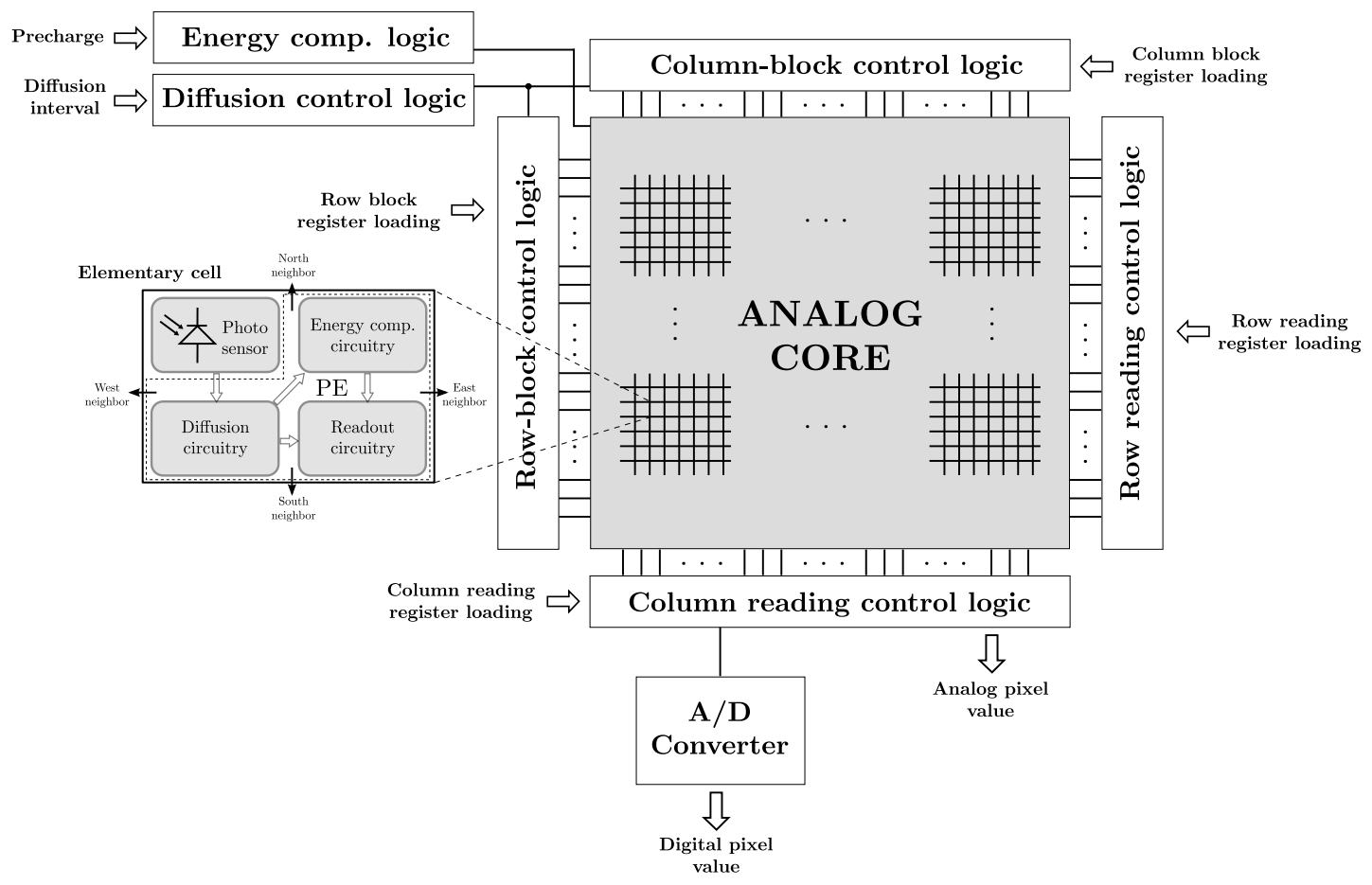

Figure 5.1: Floorplan of the prototype chip. 


\begin{tabular}{cc}
\hline \hline Technology & $0.35 \mu \mathrm{m}$ CMOS $2 \mathrm{P} 4 \mathrm{M}$ \\
\hline Vendor (Process) & Austria Microsystems (C35OPTO) \\
\hline Die size (with pads) & $7280.8 \mu \mathrm{m} \times 5780.8 \mu \mathrm{m}$ \\
\hline Cell size & $34.07 \mu \mathrm{m} \times 29.13 \mu \mathrm{m}$ \\
\hline Fill factor & $6.45 \%$ \\
\hline Resolution & QCIF: $176 \times 144 \mathrm{px}$ \\
\hline Photodiode type & n-well $/ \mathrm{p}-\mathrm{substrate}$ \\
\hline Power supply & $3.3 \mathrm{~V}$ \\
\hline Signal range & {$[1.5 \mathrm{~V}, 2.5 \mathrm{~V}]$} \\
\hline FPN & $0.72 \%$ \\
\hline PRNU (50\% signal range) & $2.42 \%$ \\
\hline Sensitivity & $0.15 \mathrm{~V} /(\mathrm{lux} \cdot \mathrm{s})$ \\
\hline Measured power consumption & $5.6 \mathrm{~mW} @ 30 \mathrm{fps}$ \\
(worst case) & $22 \times 18 \mathrm{px}$ \\
\hline Predicted power consumption & $17.6 \mathrm{~mW} @ 30 \mathrm{fps}$ \\
(worst case) & $176 \times 144 \mathrm{px}$ \\
\hline ADC throughput & $0.11 \mathrm{MSa} / \mathrm{s}(9 \mu \mathrm{s} / \mathrm{Sa})$ \\
\hline \hline
\end{tabular}

TABLE 5.1: Summary of the prototype chip features.

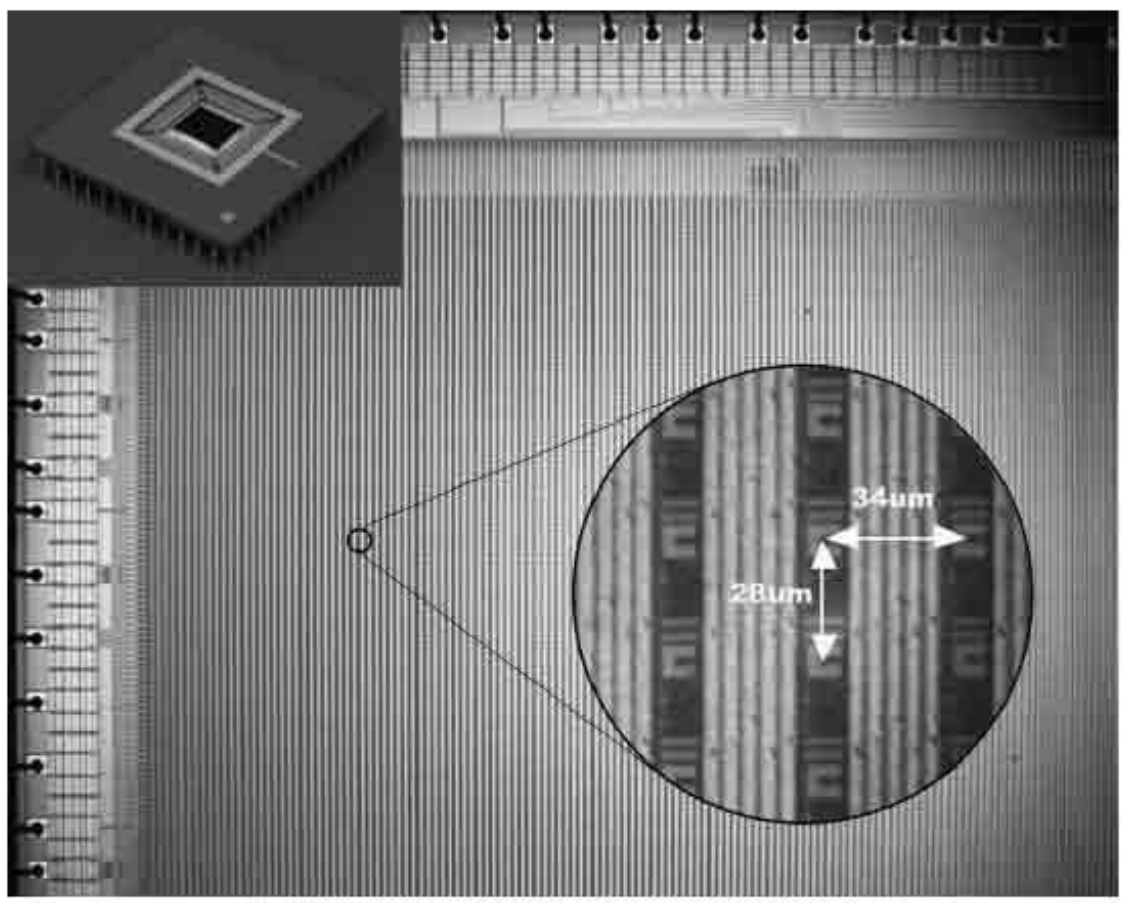

FigURE 5.2: General view and microphotographs of the FLIP-Q prototype. 


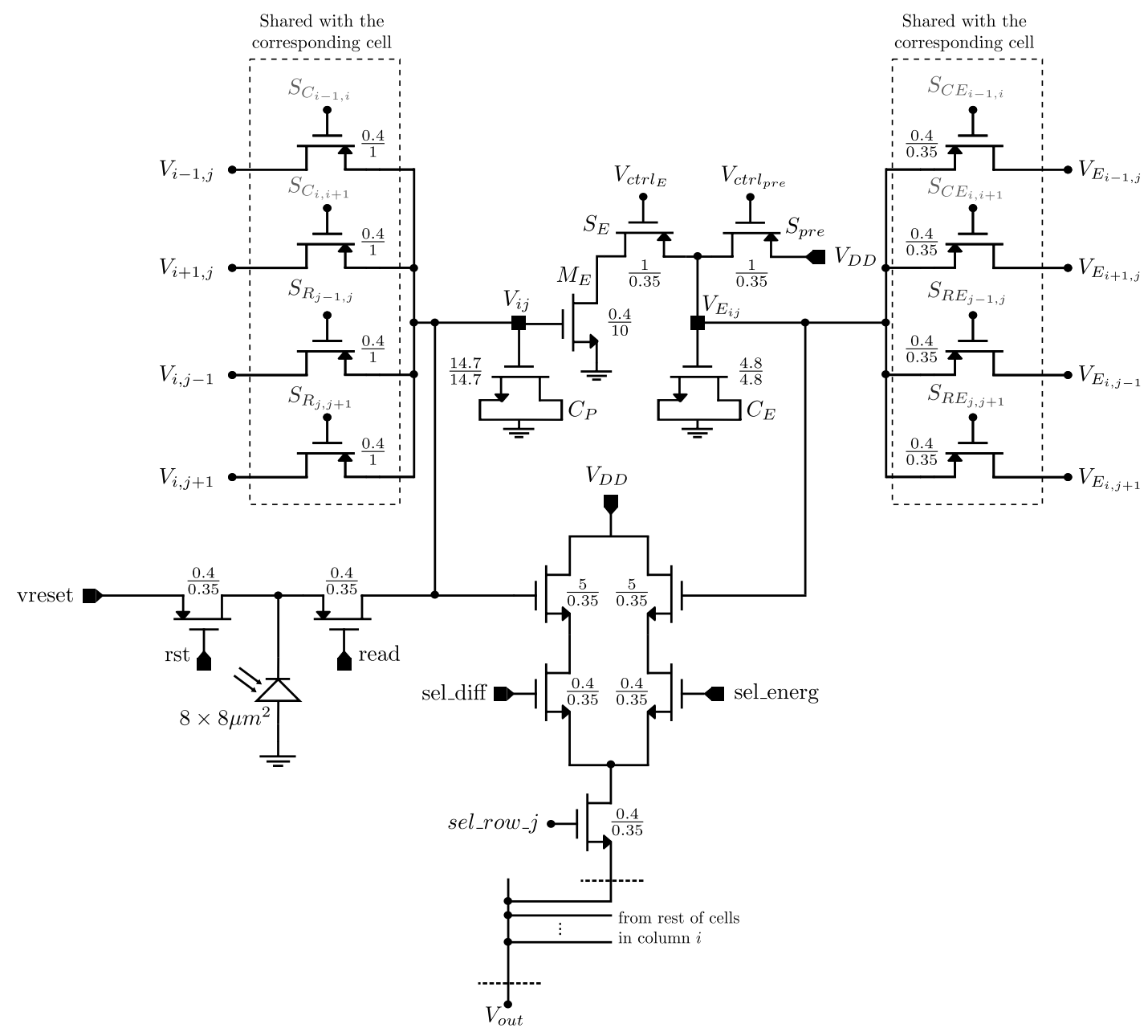

FiguRE 5.3: Elementary cell of the array.

is $2.5 \mathrm{~V}$. It can be extended to $3.3 \mathrm{~V}$ though the accuracy of the analog blocks is compromised. The control signals 'rst' and 'read' implement an electronic global shutter (see Fig. 5.4). The analog pixel value is represented by the voltage $V_{i j}$ after the integration time.

\subsubsection{Diffusion-based Gaussian filtering}

Note that, in the elementary cell (Fig. 5.3), $C_{P}$ is 4-connected to its neighbors through pMOS transistors. Each linking transistor is shared with the corresponding neighbor cell. The equivalent resistance $R_{e q}$ of these transistors along with the value of the $C_{P}$ determine the time constant $\tau=R_{e q} C_{P}$ of the resulting MOS-based RC network. Its design is based on the guidelines developed in Chapter 4. Our starting point is the expression for the width of the intrinsic Gaussian filtering performed by this network, $\sigma=\sqrt{2 t / \tau}$. Since this width depends on the quotient between the time interval which the network is permitted to evolve, $t$, and the time constant of the network, $\tau$, their values must be correlated in order to achieve a high degree of programmability over the filtering. It in turn establishes several tradeoffs for the design of 


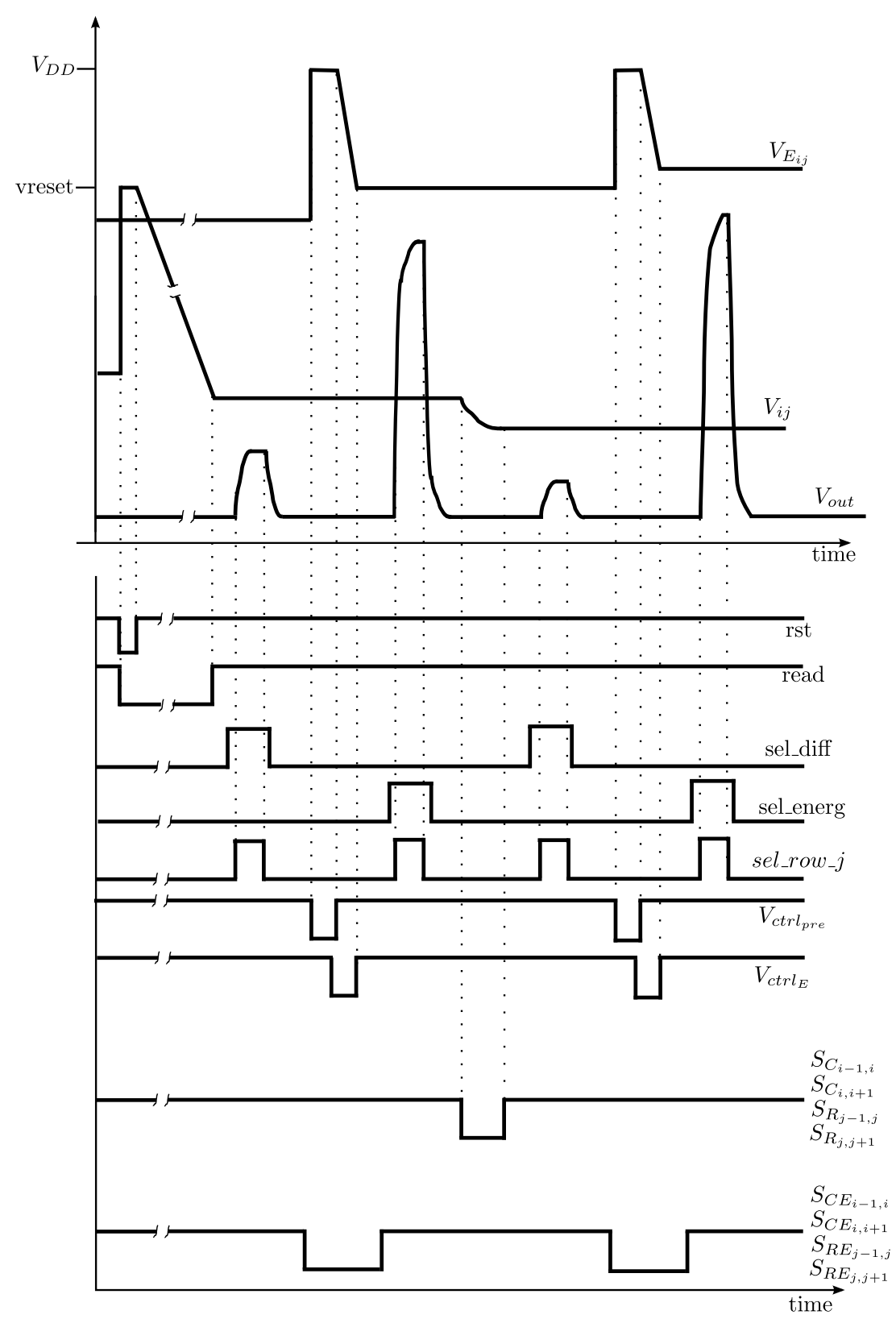

FiguRE 5.4: Timing diagram of the operation of the elementary cell.

the corresponding circuitry. On one hand, the larger the value of $\tau$ the coarser the necessary time control of the network dynamics to render a certain value of $\sigma$, making the circuitry for this control simpler. Besides, larger values of $\tau$ need more area for the implementation of the elementary transistor and elementary capacitor of the network, being therefore more robust to mismatch. On the other hand, the area consumed by these components has dramatic consequences for the size of the array as they must be included at each and every elementary cell of the focal plane. From this point of view, a small value of $\tau$ results more adequate. The problem is that a finer temporal control of the network dynamics is mandatory in such a case, forcing even the internal, i.e. on-chip, generation of the pulses which control the evolution of the grid. 


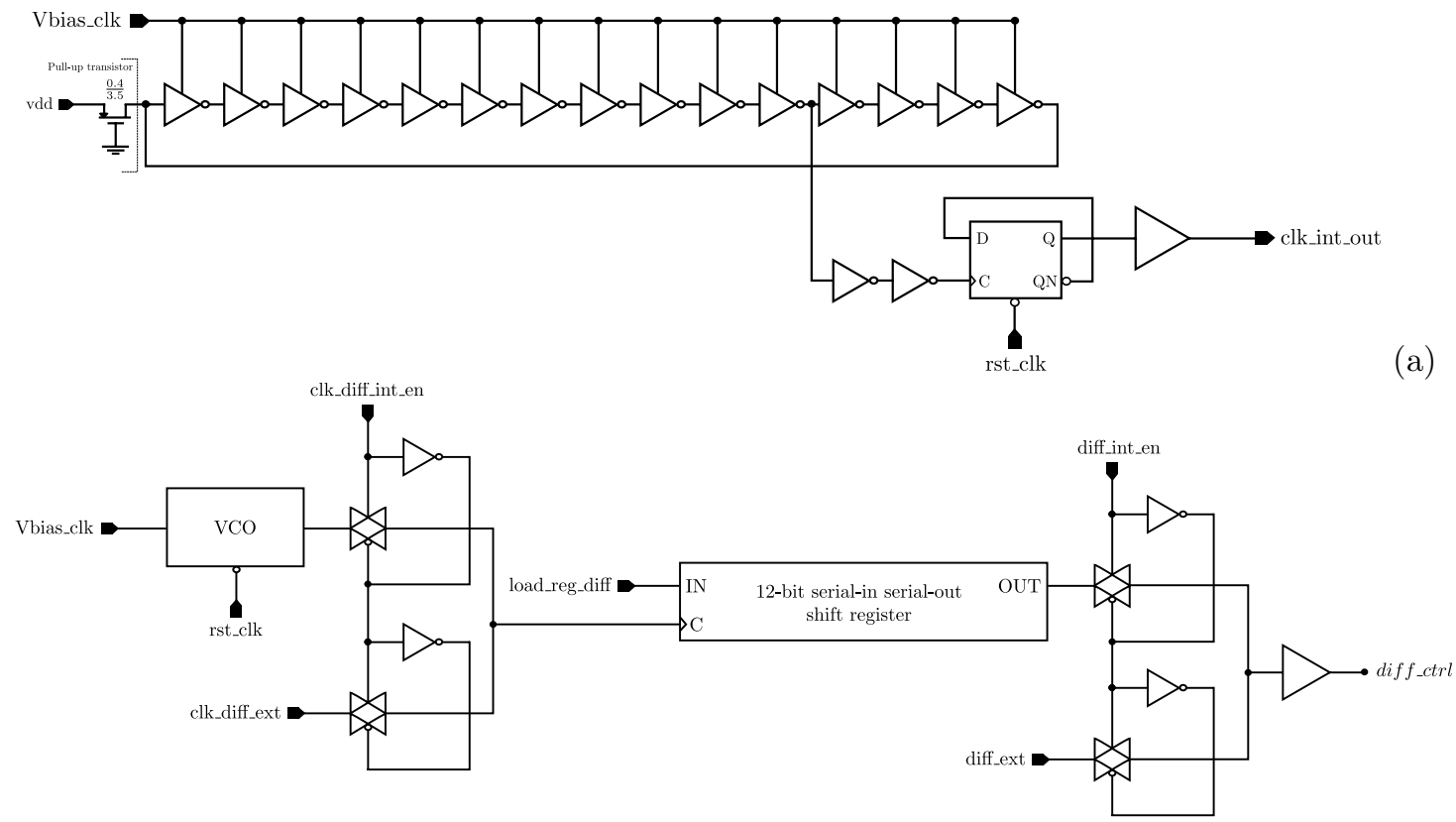

(b)

Figure 5.5: Diffusion control circuitry: (a) 15-stage inverter ring VCO and (b) diffusion control logic

Otherwise, propagation delays could distort the resulting filtering.

Taking into account all these considerations, we decided to reduce the value of $\tau$, and consequently the size of the focal-plane processing array, as much as possible. This means that a fine control of $t$ is mandatory. The hardware implemented for this fine control comprises two blocks. The first block is a VCO (Fig. 5.5(a)). It consists of a ring of pseudo-NMOS inverters in which the load current is controlled by 'Vbias_clk', thus modifying the propagation delay of each stage. Frequencies up to $150 \mathrm{MHz}$ can be attained. This circuit provides an internal clock that will be employed to time pulses that add up to the final diffusion duration.

The second block of the diffusion control is a 12-stage shift register (SHR) (Fig. 5.5(b)). It will store a chain of ' 1 's indicating how many clock cycles the diffusion process will run. The clock employed for this will be either external or the already described internal $\mathrm{VCO}^{2}$. The output signal, diff_ctrl, is a pulse with the desired duration of the diffusion, $t$, that is inverted and delivered to the gates of the pMOS resistors. Thus, $t$ depends on two parameters, namely: $N_{1}$, which is the number of logic ' 1 's within the bit string, and $f_{C L K}$, the frequency of the clock. In this way, $t=N_{1} / f_{C L K}$. In order to provide some guard time for internal timing of the operation, the first two bits introduced into the SHR, that is, the first two bits defining the diffusion time, must be set to ' 0 '. Only 10 bits are therefore effectively employed to define the pulse duration. As a result, a minimum diffusion step of around $t_{m i n}=6.66 \mathrm{~ns}$ can be achieved.

\footnotetext{
${ }^{2}$ The aim of the internal VCO is to reach a better resolution of the diffusion time than an external clock. For loading the appropriate sequence into the register, an external, and slower, clock is usually preferred.
} 


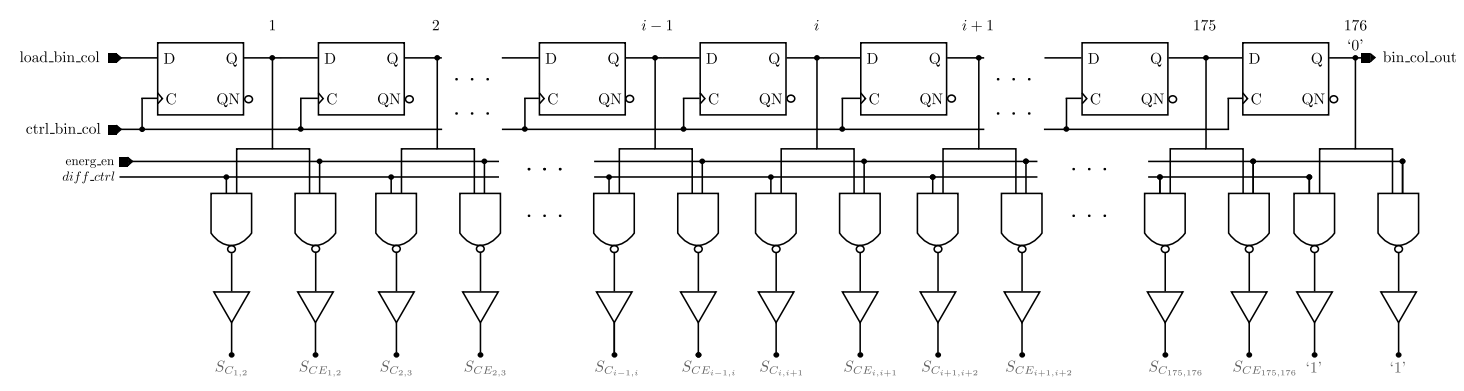

Figure 5.6: Column-wise focal-plane division control.

Once $t_{\text {min }}$ has been set, the design of the MOS-based RC network itself can be addressed. As a system specification, our objective is to implement a value of $\tau$ around one order of magnitude greater than $t_{m i n}$. This means that Gaussian filters with widths below $\sigma=1$ must be easily achieved. Really gradual scale spaces can be thus generated. Nominal $\tau$ was decided to be $85 \mathrm{~ns}$, granting margin of error to the maximum frequency reachable by the VCO and to the final value of $\tau$ physically implemented. The design procedure starts by selecting the value of the capacitor $C_{P}$. As it is the sensing capacitance, a trade-off between sensitivity and minimization of the reset error leads to $C_{P}=1 \mathrm{pF}$, implemented by a MOS-based capacitor with dimensions $14.7 \mu m \times 14.7 \mu m$. Then, continues with an automatic search for a transistor implementing $R_{e q}=$ $85 k \Omega$, exactly as done in Section 4.3 .3 for the transistor implementing $100 \mathrm{k} \Omega$. pMOS transistors are now considered due to their better ratio resistance/area. A transistor with geometric aspect $0.4 / 1$ is thus obtained for typical conditions (TM).

The key aspect of the MOS-based RC network designed from the point of view of the image processing is that the connection between any two neighbor nodes can be controlled through the gate voltage of the transistor which links them, signals $S_{C_{i-1, i}}, S_{C_{i, i+1}}, S_{R_{j-1, j}}$ and $S_{R_{j, j+1}}$ in Fig. 5.3. When off, the corresponding nodes are disconnected. When on, the linking transistor behaves as a resistor of value $R_{e q}$. This control, performed column-wise and row-wise, has two objectives. First of all, a permanent disconnection between certain consecutive columns and rows across the array determines the boundaries of the blocks in which the focal plane is divided. Secondly, a time-controlled connection between consecutive columns and rows implements a spatially-discretized diffusion process over the voltages $V_{i j}$ within the respective blocks.

\subsubsection{Block division control logic}

It comprises the column-block and row-block control logic modules in Fig. 5.1. These modules generate the appropriate selection signals to configure the image sub-blocks. Links between cells within the same block are enabled. Disabling a column/row across the array establishes one of the boundaries of the adjacent blocks. We are going to focus on the column-block control logic (Fig. 5.6) as its description is directly applicable to the row-block control logic (Fig. 5.7). The 


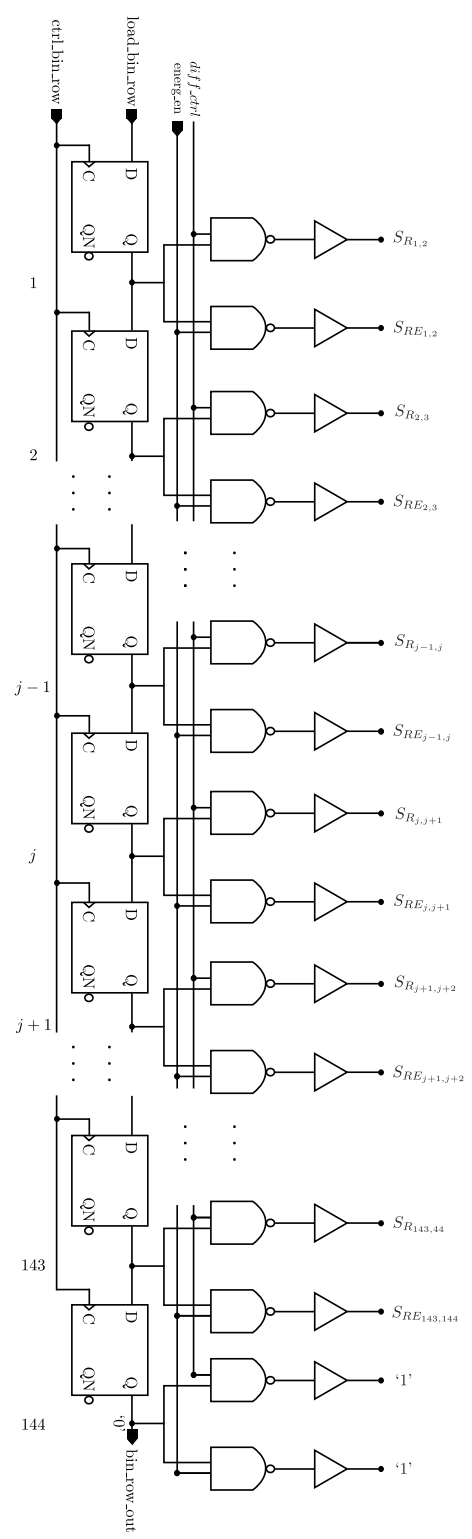

FiguRE 5.7: Row-wise focal-plane division control.

operation is based on a SHR which is externally loaded and clocked. Each bit of the register determines the link between two columns of PEs. Thus the bit ' $\mathrm{i}$ ' storing a logic value ' 1 ' determines that columns ' $\mathrm{i}$ ' and ' $\mathrm{i}+1$ ' are linked. On the contrary, the bit ' $\mathrm{i}$ ' storing a logic value ' 0 ' establishes that columns ' $\mathrm{i}$ ' and ' $\mathrm{i}+1$ ' are unlinked. This scheme allows for an easy and fast reconfiguration of the blocks by adequately shifting the patterns loaded into the registers. Besides, it is specially suited for a microcontroller as only four pins - two for the column register and two for the row register — suffice to define the focal-plane division. The internal, activehigh, signal diff_ctrl comes from the diffusion control logic (Fig. 5.5(b)). This signal controls the time interval $t$ of diffusion filtering within the blocks once the focal-plane division is set. The signal 'energ_en' enables in turn the computation of the block energy. Notice that each and every signal $S_{C_{m, m+1}}$ and $S_{C E_{m, m+1}}$ - correspondingly $S_{R_{n, n+1}}$ and $S_{R E_{n, n+1}}$ in the row-control 
logic - must be buffered in order to achieve an accurate timing of the control logic across the array. It benefits the accuracy of the processing. In fact, all the signals which must nominally reach the whole array at the same time are carefully buffered.

\subsubsection{Energy computation}

The progressive spatial filtering performed during the diffusion also allows for further simplification of the scene. Let $V_{i j}(t)$ be the voltages at the nodes of a $W \times H$ block after a certain interval of diffusion $t$. As we saw in Chapter 3, the total energy of the block is defined as:

$$
E_{T}(t)=\sum_{i=1}^{W} \sum_{j=1}^{H}\left|V_{i j}(t)\right|^{2}=\sum_{u=0}^{W-1} \sum_{v=0}^{H-1}\left|\hat{V}_{u v}(t)\right|^{2}
$$

constituting a measure of the evolution of the diffusion process. An energy-based scene representation makes the efficient segmentation of spatially-repetitive patterns possible by monitoring the value of the energy along the diffusion. Besides, the difference between the initial value of the energy and the energy after a long enough diffusion accounts for the contrast within the block considered. The higher the value of this difference, the larger the intensity changes which determine the frequency content of the block. This information allows for a first estimation of the salient regions of the scene $[\mathrm{Ni}, 2005]$.

In order to efficiently compute the block energy at the focal plane, we make use of the MOS transistor square law and the summation of the contribution of the individual pixels in the form of currents. It is implemented by the transistor $M_{E}$ (Fig. 5.3), working in saturation, the capacitor $C_{E}$, the switches $S_{E}$ and $S_{p r e}$ and MOS switches for charge redistribution which average the voltages $V_{E_{i j}}$ within the block. Firstly, as we are interested in the computation of the energy associated to the previously defined blocks of the image, the same block division as for the voltages $V_{i j}(t)$ is established by the selection signals $S_{C E_{m, m+1}}$ and $S_{R E_{n, n+1}}$ (see Fig. 5.6 and Fig. 5.7). Then, all the capacitors $C_{E}$ are precharged to $V_{D D}, 3.3 \mathrm{~V}$, by switching on both $S_{E}$ and $S_{\text {pre }}$ (see this happening twice, one before diffusion and one after diffusion, in the diagram of Fig. 5.4). Then, $S_{p r e}$ is switched off while $S_{E}$ is kept on during a time interval $T_{E}$, 20ns in our case, discharging $C_{E}$ through $M_{E}$. Once $S_{E}$ is definitely switched back off, the voltage at $C_{E}$ would be, with respect to $V_{D D}$, proportional to the pixel energy:

$$
V_{E_{i j}}=V_{D D}-\frac{T_{E}}{C_{E}} \beta\left[V_{i j}(t)-V_{t h}\right]^{2}
$$

where $V_{t h}$ is the threshold voltage and $\beta$ the transconductance parameter of $M_{E}$. However, due to the charge redistribution realized through the MOS resistors, the following value is eventually reached: 


$$
V_{E_{i j}}=V_{D D}-\frac{\beta T_{E}}{W H C_{E}} \sum_{i=1}^{W} \sum_{j=1}^{H}\left[V_{i j}(t)-V_{t h}\right]^{2}
$$

which is, again with respect to $V_{D D}$, proportional to the total energy of that block $t$ seconds after the diffusion started. The nominal signal range for $V_{E_{i j}}$ is $[2.1 \mathrm{~V}, 3 \mathrm{~V}]$. In the ideal case in which all the $M_{E}$ transistors perfectly match, the offset introduced by $V_{t h}$ will not affect the computation of the energy associated to any spatial frequency other than the dc component. In the real chip, $V_{t h}$ is subject to across-die variations, as other transistor parameters are. This induces FPN to appear. We have measured the amount of FPN present in the energy representation of each individual pixel. First, while keeping the capacitors $C_{E}$ on reset, the output is sampled several times in order to filter out the temporal noise contribution. The result is a standard deviation of $1.12 \%$ across the image referred to the full signal range of the output corresponding to the readout of the energy representation. In addition, we have allowed the capacitors to discharge for a uniform image in the middle of the range, i.e. $2.0 \mathrm{~V}$ at node $V_{i j}$, also for a number of times. Subtracting the averaged values obtained before from these later ones the standard deviation is now $7.85 \%$. It summarizes the contribution of the mismatch of $V_{t h}$, amplified by the transistor square-law, the mismatch in the transconductance of $M_{E}$, and the switching errors introduced by $S_{E}$ and $S_{\text {pre }}$, significant now as $C_{E}$ is not so large as $C_{P}$. Note that this deviation represents the worst possible case as the energy computation is hardly applied to individual pixels. Instead, it is usually employed to represent the energy content of a group of pixels where the charge redistribution taking place to obtain the final value of $V_{E_{i j}}$, Eq. (5.3), constitutes a spatial lowpass filter that reduces the influence of FPN. In such a case, only one pixel out of every block needs to be read as all the capacitors within the block will be at the same voltage.

The module generating the signals $V_{c t r l_{p r e}}$ and $V_{c t r l_{E}}$ which control respectively the precharge and the energy computation is depicted in Fig. 5.8. The most important requirement to be fulfilled is the timing of $V_{c t r l}$, that is, $T_{E}=20 \mathrm{~ns}$. To meet this specification, a simple masterslave D flip-flop clocked at 50MHz suffices. Although four flip-flops have been implemented, the signals are extracted from the last one. The three first ones are included to avoid metastability. Note that the output of the last flip-flop, which corresponds to $V_{c t r l}$, is always delayed a clock period (20ns) with respect to its input, which in turn corresponds to $V_{c t r l} l_{p r e}$. Therefore both signals come from only one, the active-low signal 'energ'. It is drawn from simulation that the energy can be obtained by setting 'energ' to ' 0 ' for $200 \mathrm{~ns}$. As a final remark, both $V_{c t r l} l_{\text {pre }}$ and $V_{c t r l_{E}}$ can be separately input for test purposes through the external signals 'pre_ext' and 'energ_ext', respectively. 


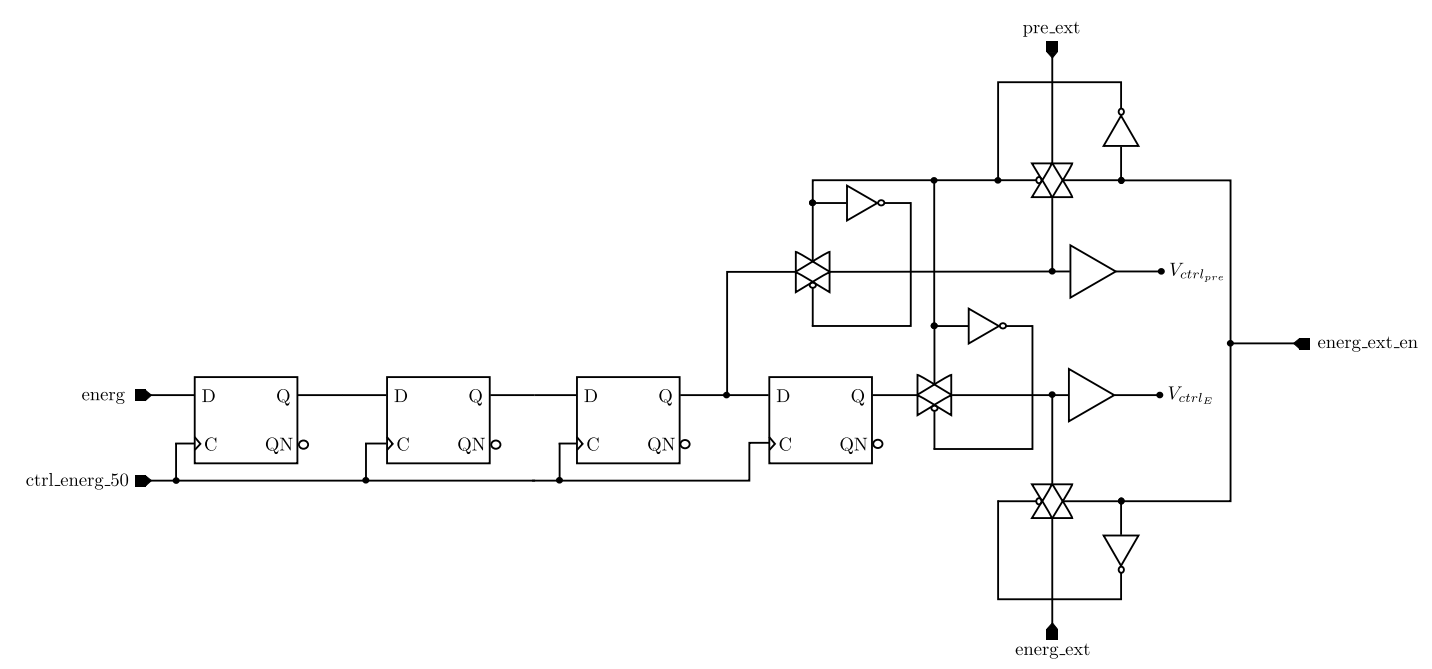

FiguRE 5.8: Generation of the signals for energy computation.

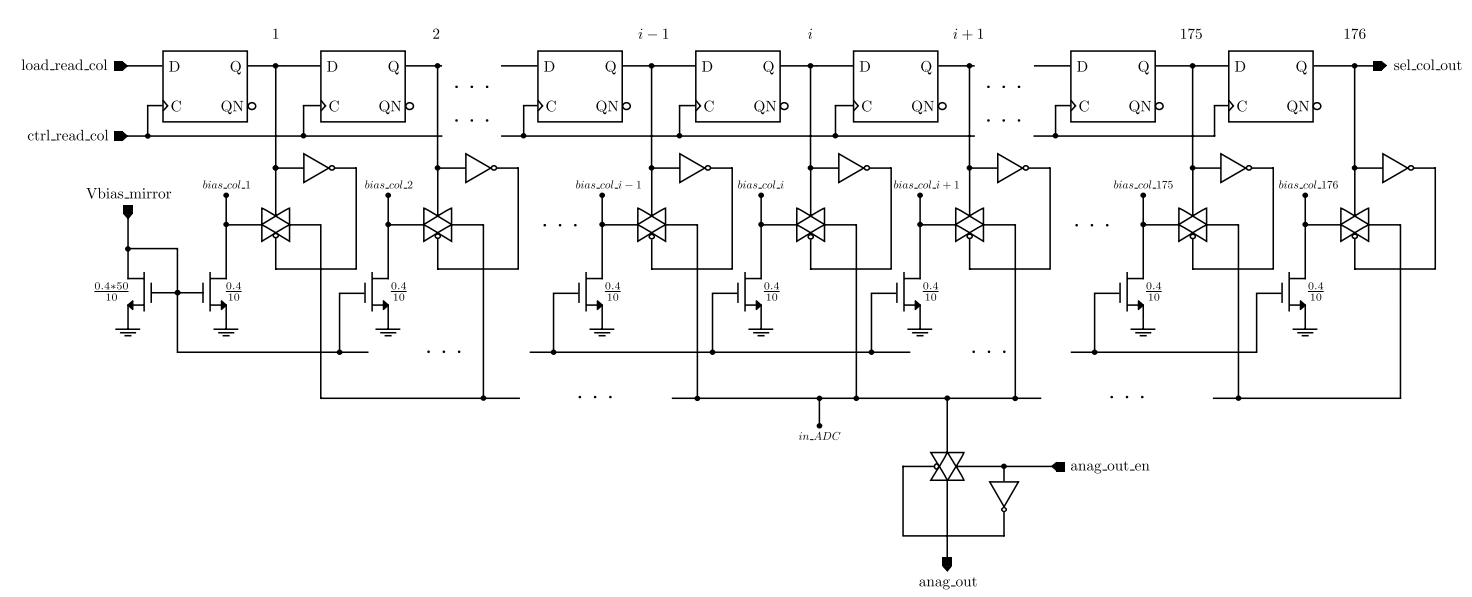

FiguRE 5.9: Circuitry for column reading.

\subsection{Readout circuitry}

\subsubsection{Reading control logic}

It comprises the column (Fig. 5.9) and row (Fig. 5.10) reading control logic in Fig. 5.1. These modules contain a serial-in/parallel-out SHR where all the bits are set to ' 0 ' but one set to ' 1 '. The bits set to ' 1 ' enable the corresponding pixel value to the input of the A/D converter. In this way, all the pixels can be easily accessed by adequately shifting the bit strings within these registers. It also makes very simple the subsampling of the different possibilities of simplification of the scene. Notice that the analog raw value of the pixel can be read out if enabled by the signal 'anag_out_en' in Fig. 5.9. The pixel values come from the corresponding source follower at the elementary cell whose current biasing is performed in the column reading module by a nMOS mirror with a ratio $50 / 1$. This current, nominally $150 \mu \mathrm{A}$, can be provided by connecting 


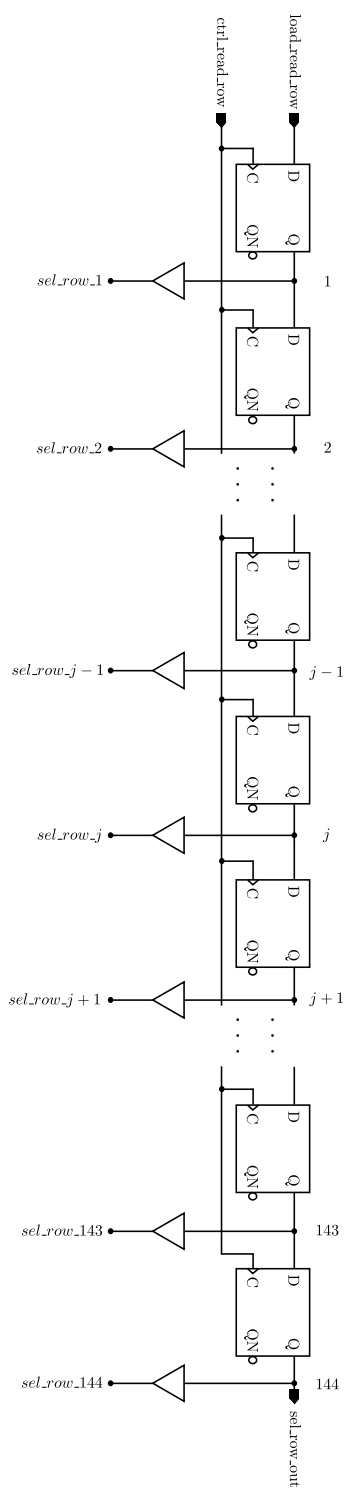

Figure 5.10: Circuitry for row reading.

$3.3 \mathrm{~V}$ to 'Vbias_mirror' through a $10 \mathrm{k} \Omega$ resistor and is translated into $3 \mu \mathrm{A}$ to bias the source followers. It permits to drive the input capacitance of the $\mathrm{A} / \mathrm{D}$ converter in such a way that the pixel value is ready to be converted not longer than $1 \mu$ s since it was selected to be read out.

\subsubsection{A/D Conversion}

The A/D conversion is carried out by means of an 8-bit SAR A/D converter belonging to the set of Analog Standard Cells of the CMOS process in which the prototype was fabricated. The main characteristics of this converter are summarized in Table 5.2 and its symbol is depicted in Fig. 5.11. A timing diagram describing its operation can be found in Section 5.7. 


\begin{tabular}{cc}
\hline \hline Conversion time & $9 \mu \mathrm{s}$ \\
\hline Clock frequency & $1 \mathrm{MHz}$ \\
\hline Input capacitance & $3 \mathrm{pF}$ \\
\hline$D N L$ & $\pm 0.25 \mathrm{LSB}$ \\
\hline$I N L$ & $\pm 0.25 \mathrm{LSB}$ \\
\hline ENOB & $7.6 \mathrm{bits}$ \\
\hline Input voltage range & {$[0 \mathrm{~V}, 3.3 \mathrm{~V}]$} \\
\hline Area & $250.6 \times 159.2 \mu m^{2}$ \\
\hline Power consumption & $1.2 \mathrm{~mW}$ \\
\hline Throughput & $0.11 \mathrm{MSa} / \mathrm{s}$ \\
\hline \hline
\end{tabular}

TABLE 5.2: Summary of the A/D converter.

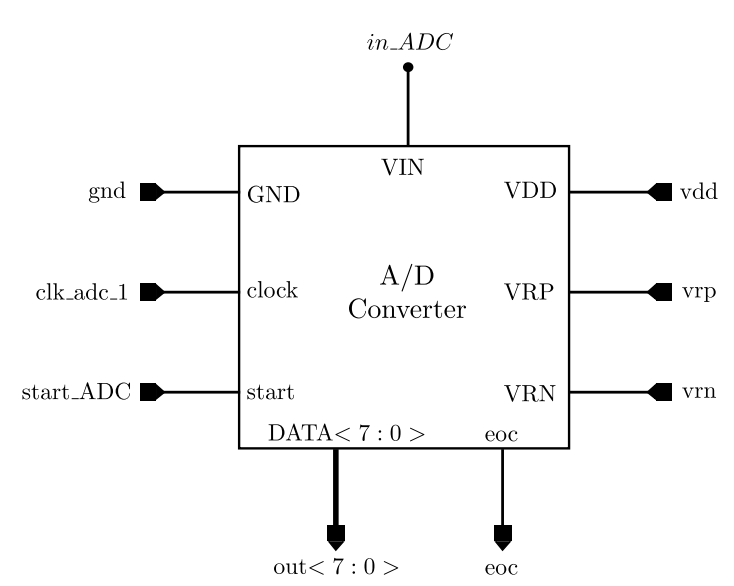

Figure 5.11: Symbol for the A/D Converter.

\subsection{Experimental results}

We present next experimental results obtained with a laboratory setup like that of Fig. 5.12. A test board was set up for housing one of the prototype samples and potentiometers associated with the biasing signals. Four signal pods enable the programming of the prototype through a pattern generator. Finally, a logic analyzer receives the resulting digital flow from the chip and stores it for further processing and visualization with MATLAB ${ }^{\circledR}$.

\subsubsection{Calibration of the time constant for diffusion}

We demonstrated in Chapter 4 the robustness to mismatch of MOS-based RC networks with elementary MOS resistors carefully designed to emulate a targeted resistance. In fact, during the test of the chip, no significant signs of anisotropy in the diffusion due to mismatch has been appreciated. However, the equivalent resistance implemented by the elementary MOS resistor 


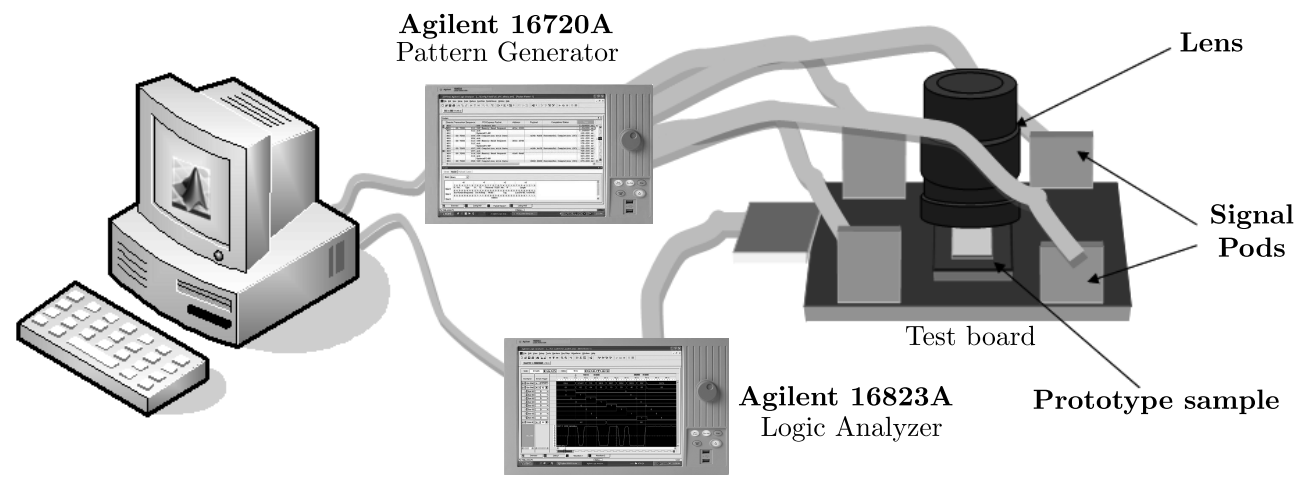

FIGURE 5.12: Laboratory setup for prototype testing.

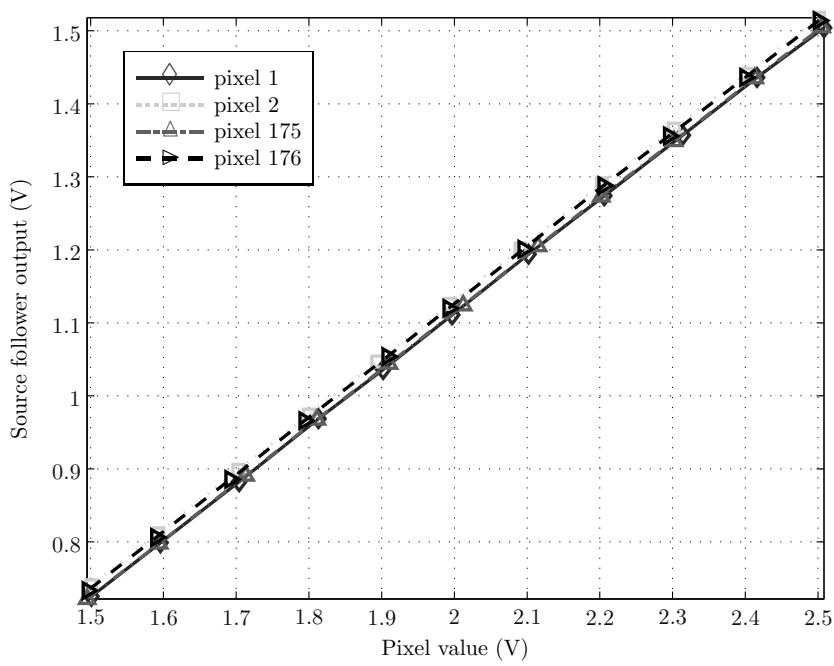

FIgURE 5.13: Characterization of the four followers involved in the calibration of $\tau$.

is quite sensitive to process parameter deviations from sample to sample. The reason is that, as was briefly commented in Section 4.3.1, $S_{n_{o p t}}$ varies, due to its dependence of $k_{n}$, across the design space delimited by the corners of the process. Or, equivalently, for a fixed $S_{n_{\text {opt }}}$, the resistance implemented by the transistor varies according to the variations in $k_{n}$. In our case, the value of the resistance, nominally $85 \mathrm{k} \Omega$ for typical conditions, ranges from $49 \mathrm{k} \Omega$ (WP corner) to $148 \mathrm{k} \Omega$ (WS corner) according to simulation. The problem therefore is to know the exact value of $R_{e q}$ in a specific sample. In other words, we need a calibration process of $\tau$. The target of this calibration is to obtain an experimental value that can be employed to generate off-chip the response of an ideal $\mathrm{RC}$ network. If the nominal value of the time constant, $85 \mathrm{~ns}$, is employed instead of the measured $\tau$, the response of the chip will greatly deviate from the ideal response. In order to disaggregate errors due to other causes, the actual $\tau$ implemented by the chip must be therefore measured. Thus, the actual width of the implemented Gaussian filter, and consequently, the goodness of the approximation, can be accurately determined.

The calibration process consists of measuring the evolution of the voltage at two coupled pixels 


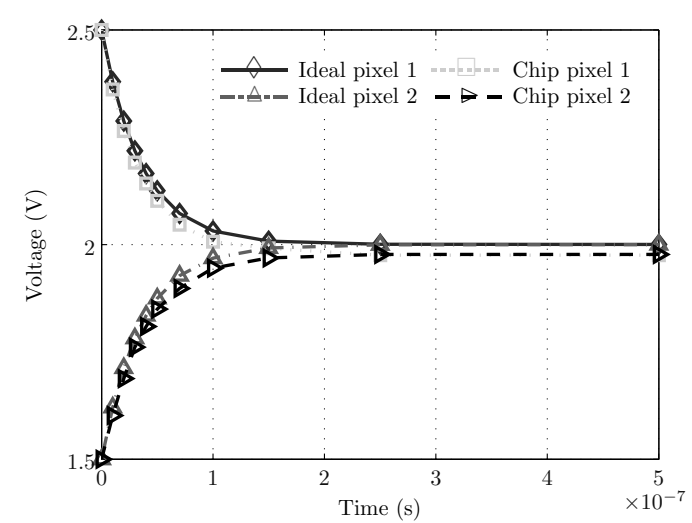

(a)

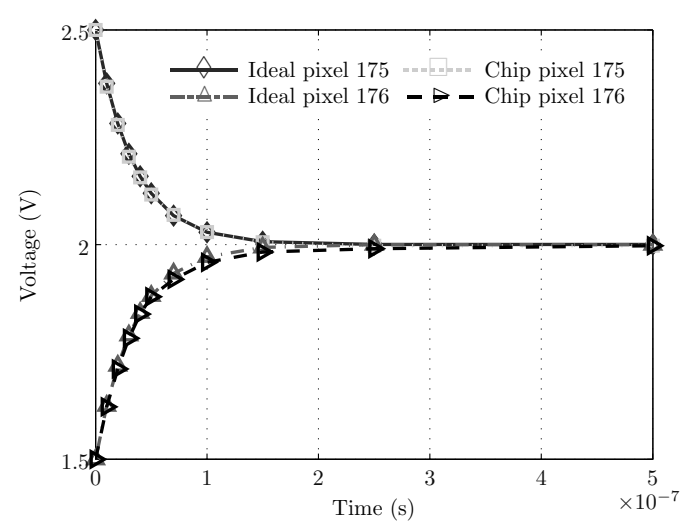

(b)

Figure 5.14: Calibration of $\tau$ at the upper left corner (a) and upper right corner (b).

whose initial values can be externally set. There is a pair of accessible pixels at each side of the array — 'pixel 1' and 'pixel 2' at the left side, 'pixel 175' and 'pixel 176' at the right side — in order to take into account variations along the die. Before testing any dynamic magnitude, each pixel's source follower is characterized in order to extract deviations introduced by the buffer from measurements of $V_{i j}$ node. The result of this characterization is depicted in Fig. 5.13. For each pair, the initial voltages are set to $V_{\min }$ and $V_{\max }$ and then successive diffusion steps are programmed until reaching the steady state. According to the results of Chapter 4, the resistance $R_{e q}$ best emulated by the MOS resistor is its instantaneous resistance when the sum of the drain and source voltages equals $V_{\min }+V_{\max }$. An ideal diffusion between a node set to $V_{\min }$ and another one set to $V_{\max }$ meets this at every time instant. Therefore, its dynamics should be perfectly emulated by the MOS-based diffusion. Obviously, second-order effects will cause deviations, but a least square fitting of the pixel values read out from the chip with respect to this ideal diffusion varying $\tau$ will permit to know the average $\tau$, similarly to how the average resistance was obtained in Section 4.3.3. The result for the upper left corner is depicted in Fig. 5.14 (a). Here, the evolution of the voltages $V_{11}$ (Chip pixel 1) and $V_{12}$ (Chip pixel 2) are compared with the evolution of the corresponding nodes of an ideal network (Ideal pixels 1 and 2 ) implementing the $\tau$ obtained from the error minimization, i.e. $\tau=72.4 \mathrm{~ns}$. A RMSE of $2.26 \%$ is obtained for this $\tau$. In the upper right corner, Fig. 5.14 (b), a minimum RMSE of $0.58 \%$ is similarly reached for $\tau=69.8 \mathrm{~ns}$. The value of $\tau$ that will be employed for the comparisons from now on will be the average of the extracted values, $\tau=71.1 \mathrm{~ns}$.

\subsubsection{Scale space}

Once $\tau$ is calibrated, any on-chip scale space can be compared to its ideal counterpart obtained by solving the spatially-discretized diffusion equation. A single image is captured to be the initial image of both the on-chip scale space and the ideal scale space calculated off-chip. This 


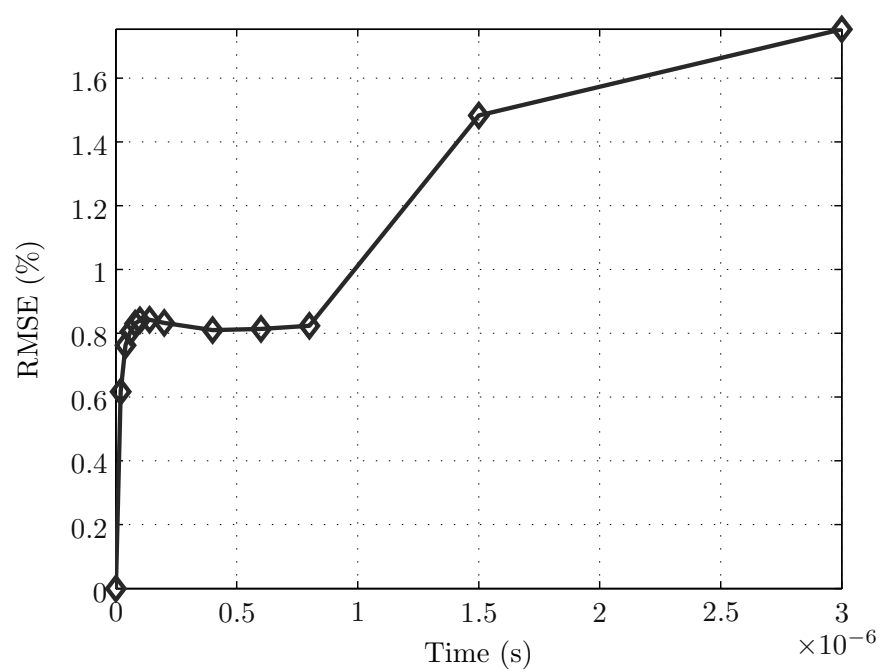

FiguRE 5.15: RMSE for the on-chip scale space with respect to the ideal case

capture is affected by a $0.72 \% \mathrm{FPN}$. It has been calculated by averaging a set of readings of the whole array without photocurrent integration, in order to skip temporal fluctuations, and then computing the standard deviation across the image. No FPN removal circuit is included in the chip, neither has been performed off-chip. Back to the scale space, the on-chip scale space is generated by applying successive diffusion steps to the original captured image. After every step, the image is converted to digital and delivered to the test instruments to be compared with the ideal image, generated by MATLAB ${ }^{\circledR}$, in terms of the RMSE (Fig. 5.15). Some of the diffusion steps are represented in Fig. 5.16 (first row) and compared to the ideal images (second row). The last row contains a pictorial representation of the error, normalized in each case to the highest measured error on an individual pixel, which are $0 \%, 24.99 \%, 19.39 \%, 6.17 \%, 3.58 \%$ and $6.68 \%$, respectively. Note that these large errors on certain pixels have little qualitative effect over the images. It can also be seen how noise eventually becomes dominant at coarse scales. Keep in mind that readout noise is present at the initial image of both scale spaces, but it is only added to each subsequent image of the on-chip scale space because of the readout mechanism. It means that while the initially stored noise, spatial and temporal, is progressively filtered in the ideal scale space, it is resampled for each picture of the on-chip scale space. As a consequence, there is an increase in the error for a sufficiently large diffusion duration. The key point here is that the accuracy of the processing predicted by simulation is very close to that of the first images of the scale space, where noise is not dominant yet. Besides, the error is kept under a reasonable level despite no FPN removal is carried out. 

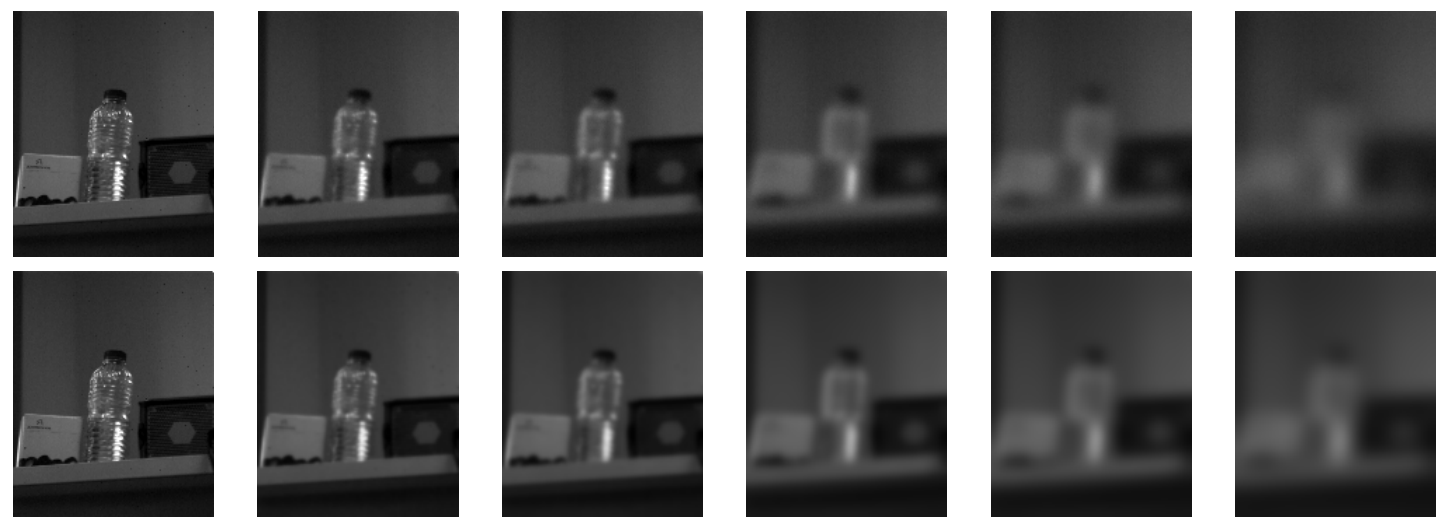

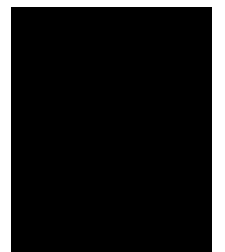

$t=0 \mathrm{~ns}$

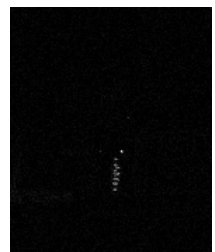

$t=40 \mathrm{~ns}$

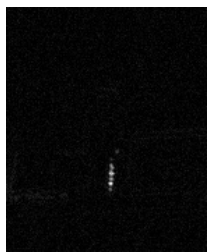

$t=100 \mathrm{~ns}$

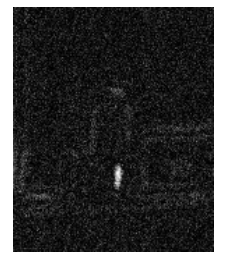

$t=400 \mathrm{~ns}$

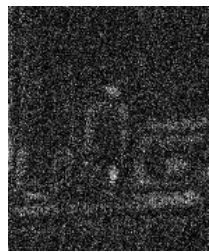

$t=800 \mathrm{~ns}$

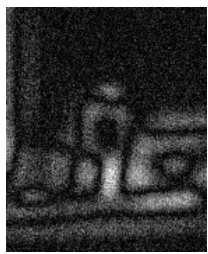

$t=1500 \mathrm{~ns}$

Figure 5.16: Scale spaces along time. The first row corresponds to the on-chip scale space, the second one corresponds to the ideal scale space and finally the third one corresponds to their normalized difference.

\subsubsection{Gaussian pyramids}

The control flow for the generation of Gaussian pyramids is very simple: (1) after image capture, the diffusion time is set to match the required scale; (2) diffusion is realized; (3) the resulting image is subsampled at the appropriate rate, 2, 4, etc.; (4) go back to (1) and set the diffusion time to match the following scale, but taking into account that the stored image is already filtered. As an example, consider the scale space described in the previous section, where $\tau=71.1 \mathrm{~ns}$. At $t=40 \mathrm{~ns}$, the components of the spatial Fourier transform at the highest vertical and horizontal frequencies, denoted respectively as $(u, v)=(M / 2,0)$ and $(u, v)=(0, N / 2)$, suffer a decrease on their magnitude by a factor of 0.1050 - we have applied Eq. (3.13) with $\tau=D^{-1}$. This means that their energy is reduced to just a $1.10 \%$ of its value at $t=0$, so they have lost nearly $99 \%$ of their energy. It means that a subsampling factor equal to 2 can be applied over the vertical and horizontal dimensions of the image without significant loss of information. For $t=80 \mathrm{~ns}$ (not shown in Fig. 5.16), both components $(u, v)=(M / 2,0)$ and $(u, v)=(0, N / 2)$ have been even more attenuated and, additionally, $(u, v)=(M / 4,0)$ and $(u, v)=(0, N / 4)$ have also lost around the $99 \%$ of their energy. In this case, a subsampling factor equal to 4 can be applied without losing relevant information. The resulting pyramids for this one and other scale space generated on-chip are depicted in Fig. 5.17. Subsampling is realized during readout by making use of the capabilities for random access to the pixel values implemented in the chip. 

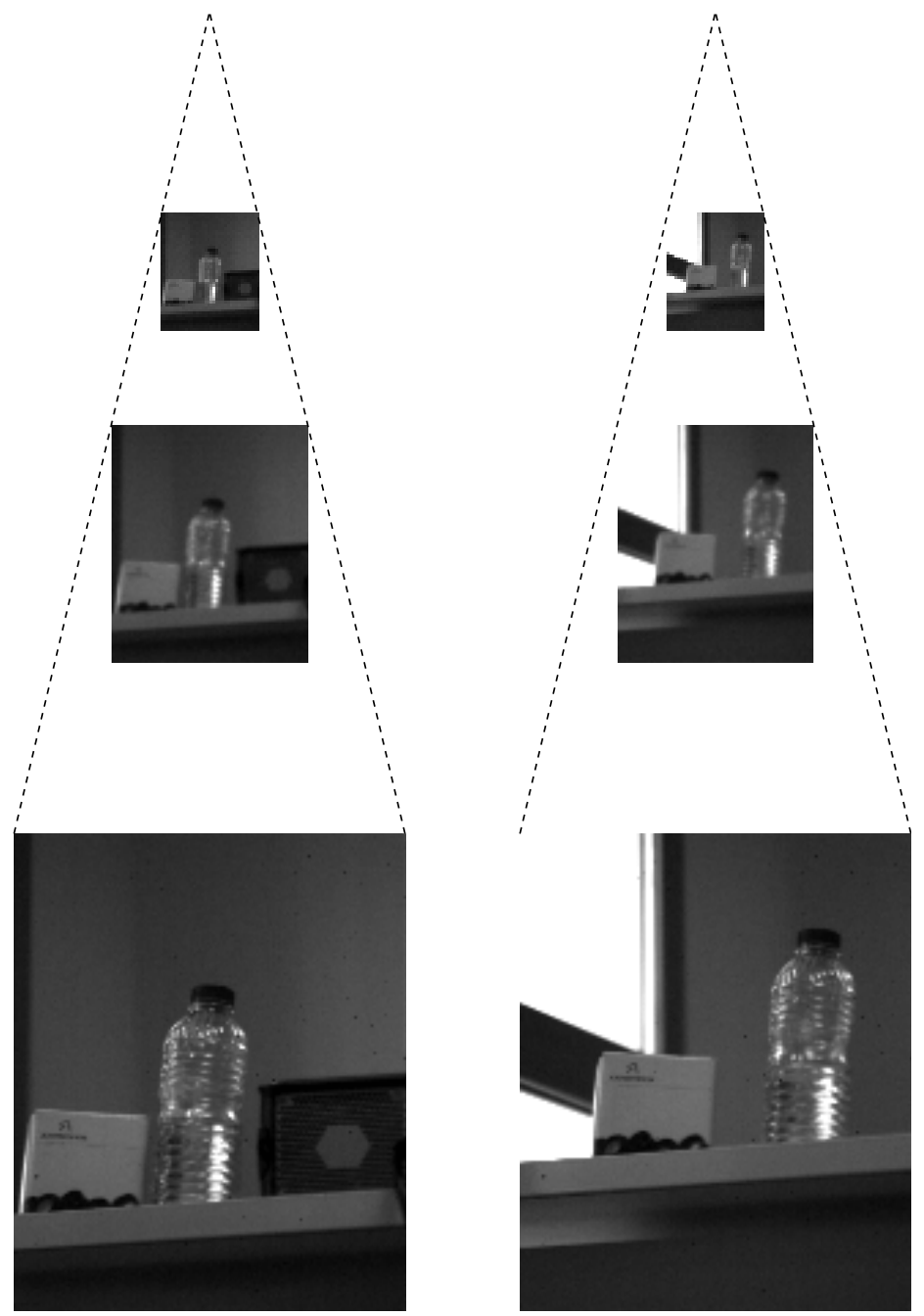

Figure 5.17: Pyramid representation of two on-chip scale spaces.

\subsubsection{Multiresolution scene representation}

The reconfigurability of the array together with the possibility of carrying out charge redistribution within each block render the representation of a scene at different resolutions extremely flexible. Several examples directly extracted from the chip can be seen in Fig. 5.18. All the images but the last one correspond to different versions of regular focal-plane divisions. The last image represents a progressive coarse-to-fine division. All these scene representations are available immediately after photointegration. Apart from the exposure time, no extra time and no extra power are required to obtain them if the focal plane subdivision is already set.

\subsubsection{Image pre-distortion for reduced kernel filtering}

We have first operated in this case on images corresponding to pictures of 'Lena' and 'Baboon' displayed at a computer screen and then captured by the chip, as depicted in Figs. 5.19 and 


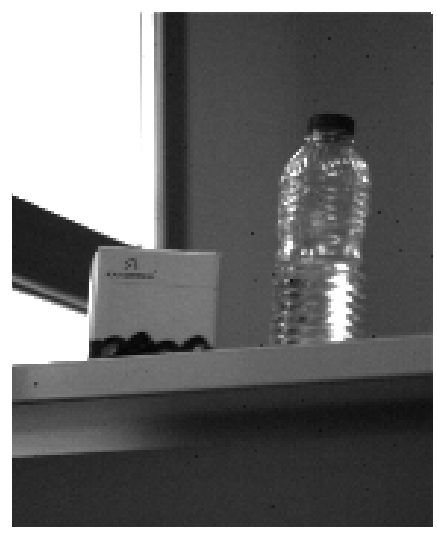

Original image

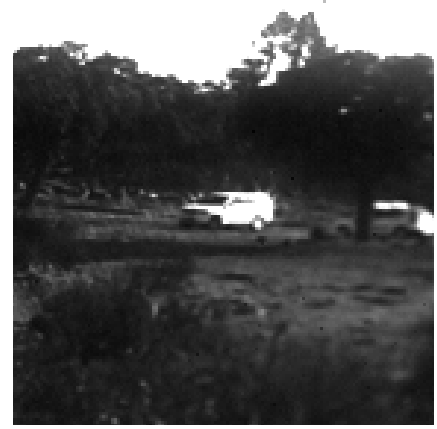

Original image

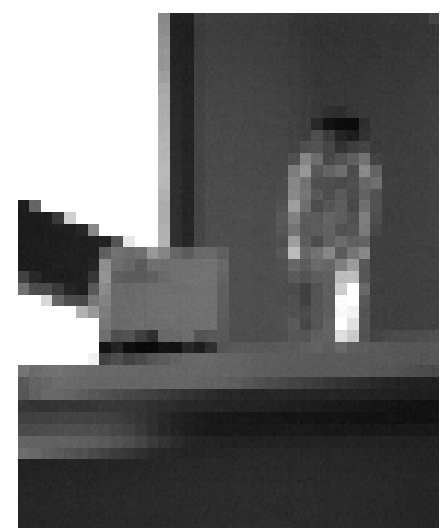

$4 \times 4 \mathrm{px}$

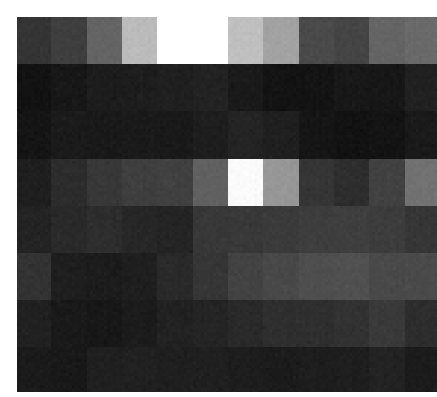

$12 \times 16 \mathrm{px}$

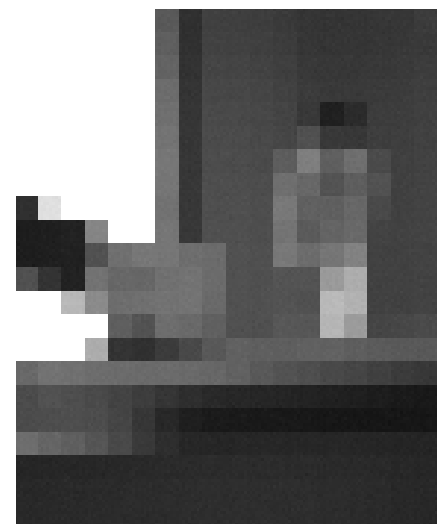

$8 \times 8 \mathrm{px}$

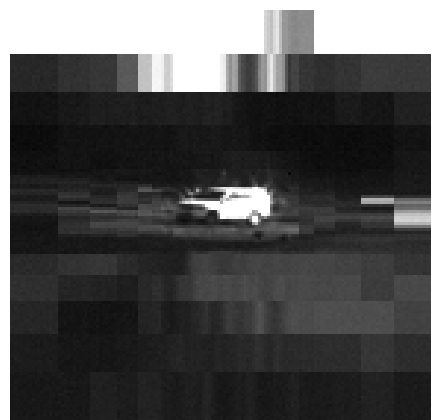

Progressive focal-plane division

FIGURE 5.18: Examples of multiresolution scene representation

5.20. Artifacts due to the screen grain can be observed. In order to reproduce the processing described in Section 3.4, we proceeded as follows: first we took a snapshot of the computer screen, either showing 'Lena' or the 'Baboon', read the full-resolution image out and performed the corresponding image filtering off-chip as a reference. This is shown in the first row of Figs. 5.19 and 5.20. Then we grouped the pixels at the focal plane as represented in Fig. 3.8(b) in order to form the half-resolution image, read it out and applied, off-chip, the filters by convolution with the $3 \times 3$ kernels. Finally, we shifted the pixel grouping as depicted in Fig. 3.8(c)-(d) and read out the pre-distorted half-resolution image to apply, also off-chip, the $2 \times 2$ kernels. The accuracy of the results is evidenced by the comparison of the two filtered versions of the image. The resulting images are perceptually equivalent. RMSE values are always below $1 \%$ in the binomial experiment, and below $4 \%$ in the application of the two Sobel filters. This is consistent with the fact that edge detection is the result of the off-chip application of two masks plus the computation of the absolute gradient value, what contributes to error spreading.

We have also completed the binomial filtering at the focal plane on another different image, Fig. 5.21, by shifting back the pixel grouping as described in Fig. 3.9. In fact, we have repeated the operation several times and the result after each step has been compared to the ideal off-chip 
equivalent filtering, as depicted in Fig. 5.22. The measured RMSE with respect to the ideal offchip operation is $1.12 \%, 1.39 \%, 1.55 \%$ and $1.69 \%$ respectively whereas the maximum individual pixel error detected at each step is $3.17 \%, 3.83 \%, 3.69 \%$ and $3.82 \%$ respectively.
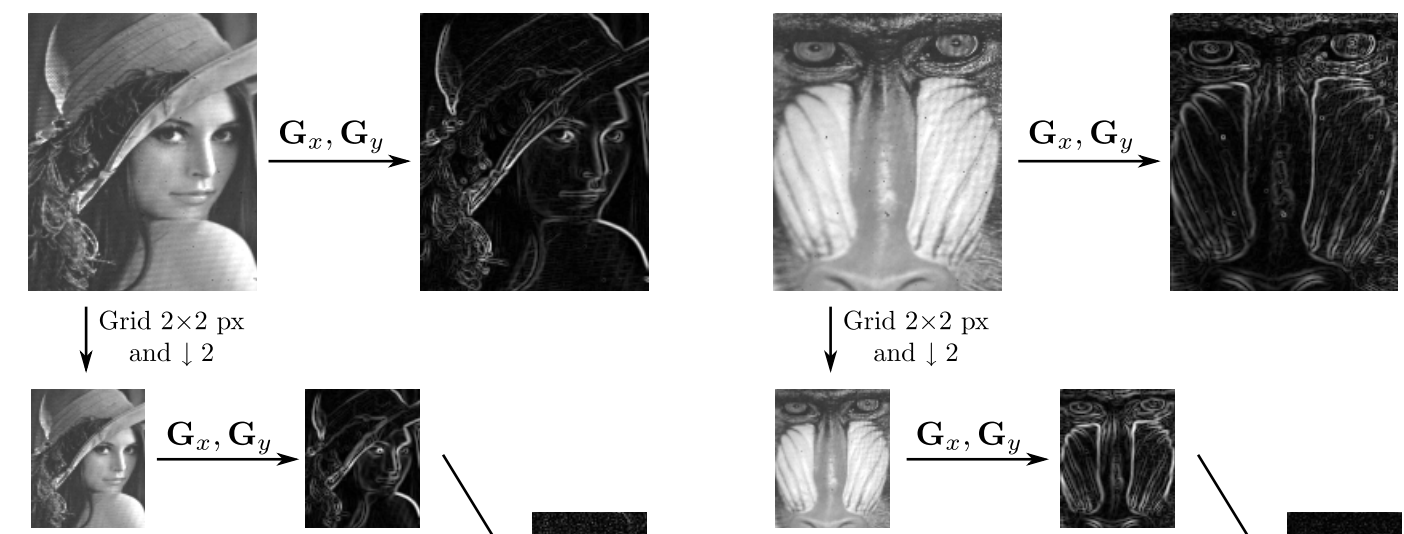

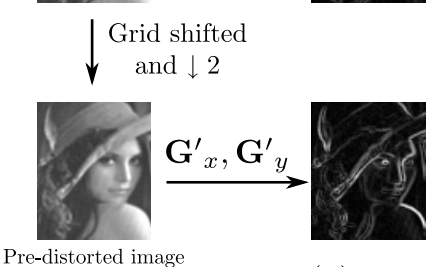

(a)

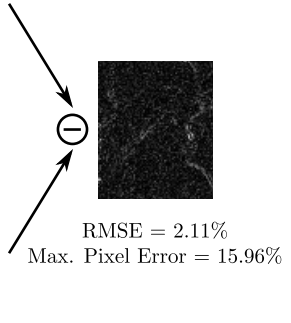

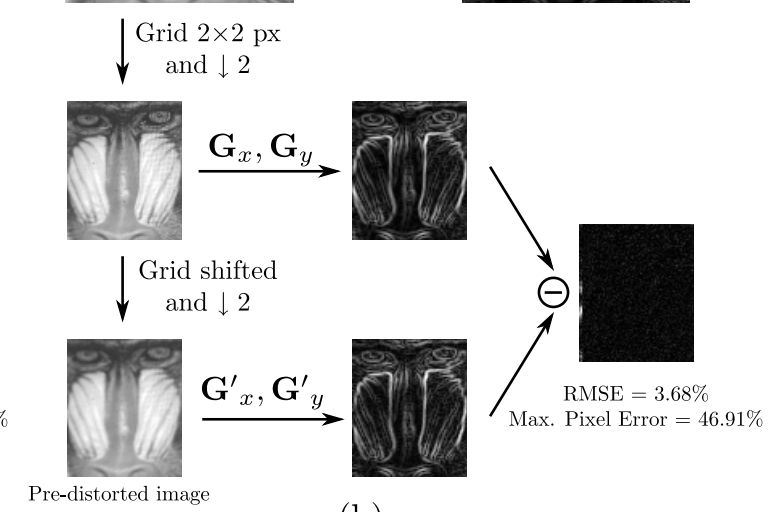

(b)

Figure 5.19: Results obtained by applying $\mathbf{G}_{x}$ and $\mathbf{G}_{y}$ to (a) 'Lena' and (b) 'Baboon' images captured by the chip, and $\mathbf{G}_{x}^{\prime}$ and $\mathbf{G}_{y}^{\prime}$ to the on-chip pre-distorted versions. The kernels $\mathbf{G}_{x}$, $\mathbf{G}_{y}, \mathbf{G}_{x}^{\prime}$ and $\mathbf{G}_{y}^{\prime}$ are defined in Eq. (3.20)

\subsubsection{Energy-based scene representation}

This primitive has been satisfactorily tested by segmenting salient regions. The results are depicted in Fig. 5.23. In these scenes, the focal plane was divided into blocks of $8 \times 8$ px. The total energy without any filtering, $V_{E_{i j}}$, and the remaining energy after a long enough diffusion, $V_{E_{i j, D C}}=V_{E_{i j}}(t \rightarrow \infty)$, were computed within every block. Thanks to the parallelism in the processing implemented by the array, the first computation took around 225ns for the whole image while the second one, including the time interval of diffusion, around 1.2us. Once $V_{E_{i j}}$ and $V_{E_{i j, D C}}$ were extracted from the chip, $V_{E_{i j}}-V_{E_{i j, D C}}$ was calculated off-line for each block and normalized to its maximum value across the image. The same computations were ideally performed with MATLAB ${ }^{\circledR}$ over the original image. The accuracy of the chip for this operation is noticeably inferior than for the scale space generation. The RMSE for the first example is $8.5 \%$ whereas for the second one is $10.9 \%$, with respect to the ideal processing. The main source of error is the signal compression taking place at the generation of the energy representation. We 


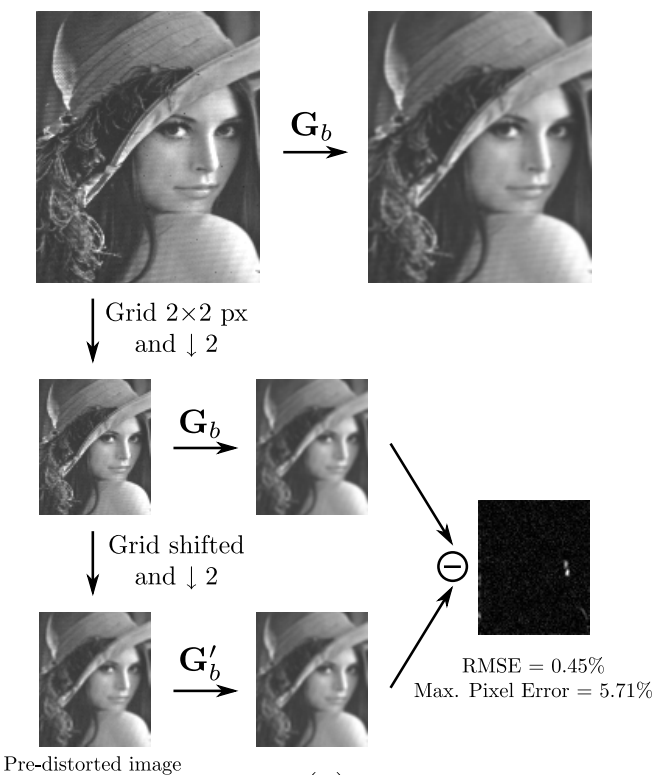

(a)
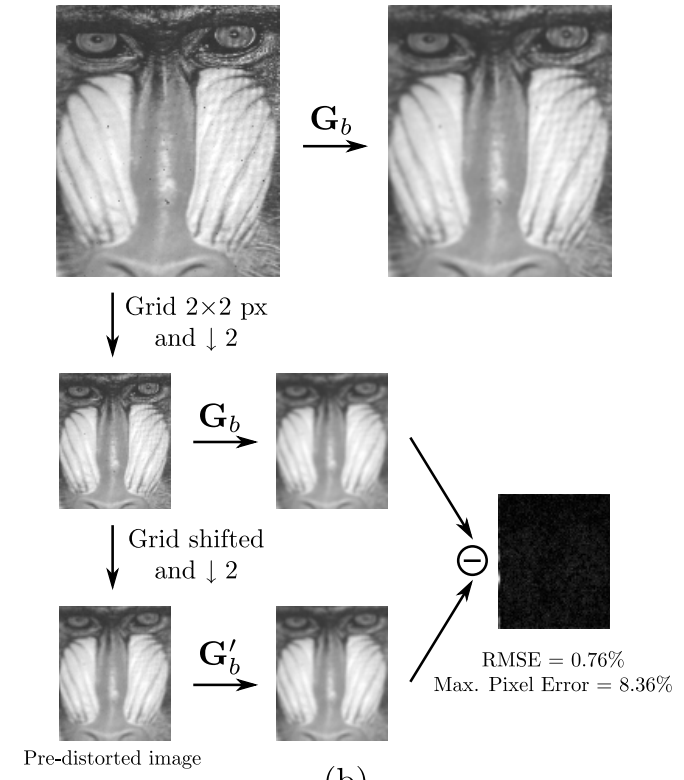

(b)

Figure 5.20: Results obtained by applying $\mathbf{G}_{b}$ to (a) 'Lena' and (b) 'Baboon' images captured by the chip, and $\mathbf{G}_{b}^{\prime}$ to the on-chip pre-distorted versions. Both $\mathbf{G}_{b}$ and $\mathbf{G}_{b}^{\prime}$ are defined in Eq. (3.21).

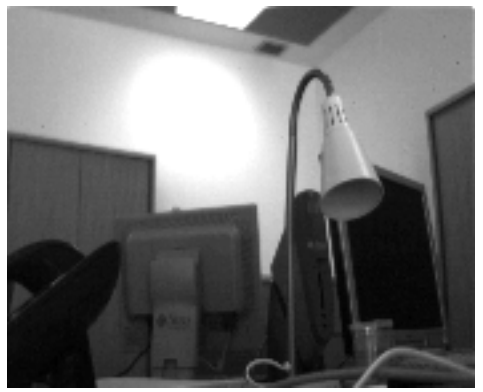

(a)

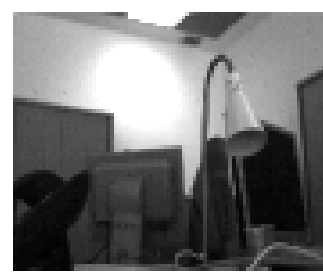

(b)

Figure 5.21: Captured image (a) and $\downarrow 2$ downsampled version (b) directly downloaded from the chip.

have started with an image represented by the pixel voltages, $V_{i j}$. Each voltage is converted to a current by $M_{E}$ according to the square-law of the MOS transistor. Therefore, any inaccuracy in the generation of $V_{i j}$ is magnified by the square-law of the transistor. Right after that the current is linearly converted to voltage by discharging capacitor $C_{E}$. As the signal ranges for $V_{E_{i j}}$ and $V_{i j}$ are similar, the signal representing the image energy is compressed compared to the signal representing the pixels' magnitude. Also second order effects, charge-injection errors, channel length modulation, transconductance and threshold mismatch, etc., become significant when millivolt range changes are usual. In any case, the absolute value of each block is not important in this case. The target of this processing is to segment the zones of the image with the largest changes of intensity, that is, the relative values among the blocks of the scene representation are the key point here. As can be seen in Fig. 5.23, the computation of the energy performed 

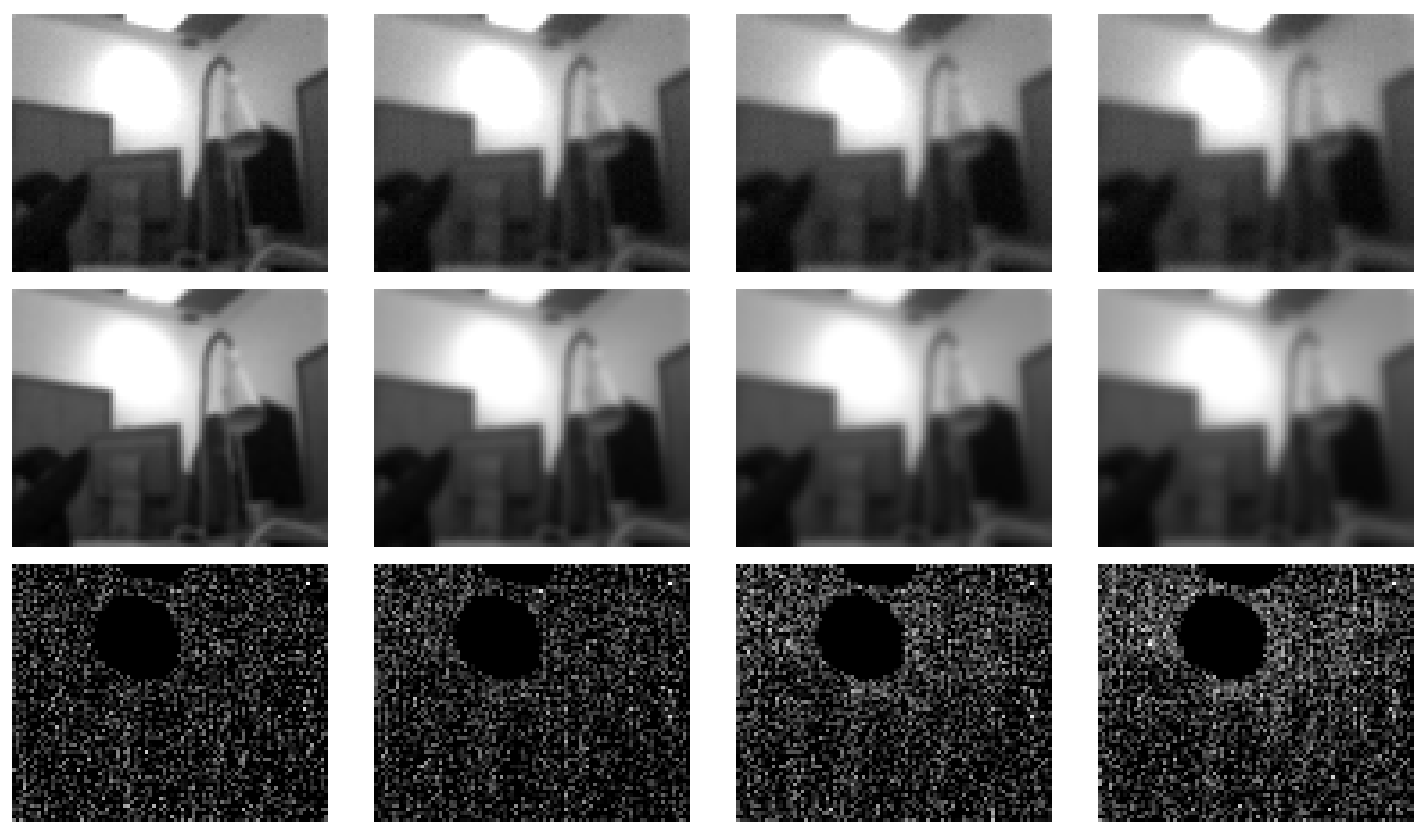

FiguRE 5.22: On-chip filtering (first row), ideal off-chip filtering (second row) and normalized difference (third row).

on-chip is capable of segmenting such zones. A subsequent step for a vision algorithm could be to realize dynamic foveation around the blocks with the largest values for a finer analysis. The outcome is depicted in Fig. 5.24 for the second scene of Fig. 5.23

\subsubsection{Power consumption analysis}

To finish this section, Table 5.3 summarizes the power consumption for different combinations of focal-plane processing, conversion and image size. All the figures are given at 30fps, although these frames are of a reduced size, as indicated in the first column, that reflects the size in pixels of the blocks delivered. Keep in mind that the chip is not intended to deliver full frame images, but reduced representations of a high informational value. The measured power include the consumption of the $\mathrm{A} / \mathrm{D}$ converter and the column buffers: $1.2 \mathrm{~mW}$ (specifications, not measured) and $0.8 \mathrm{~mW}$ (measured), respectively. As a projection of the power consumption for a full frame output, we can take into account that the current ADC and column buffers are able to deliver $0.11 \mathrm{MSa} / \mathrm{s}$ (Table 5.1), for what they need, roughly, 2.0mW. If $176 \times 144$-pixel frames are to be delivered at a rate of $30 \mathrm{fps}$, what means $0.76 \mathrm{MSa} / \mathrm{s}$, we will need 7 times more power, i. e. $14.0 \mathrm{~mW}$. Notice that the power required for focal-plane processing is the same, as it is realized full-frame in parallel. The last column of Table 5.3 accounts for this projection. It gives an idea of the efficiency of the focal-plane processing proposed. 


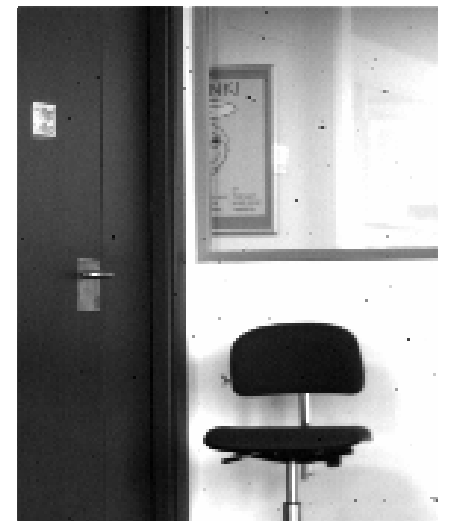

Original image

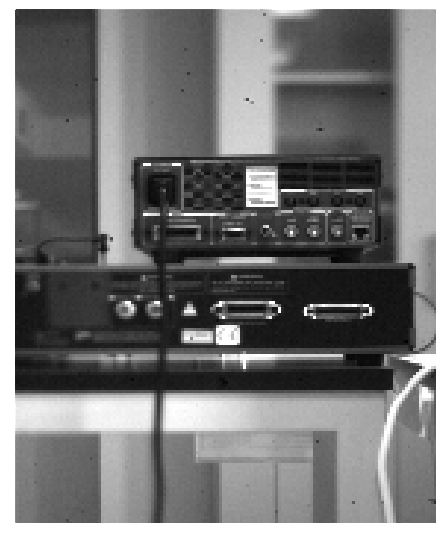

Original image

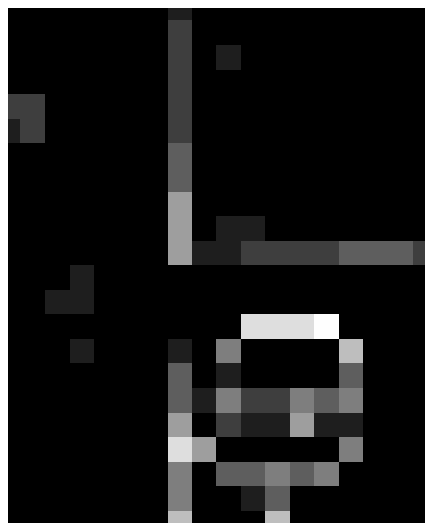

On-chip processing

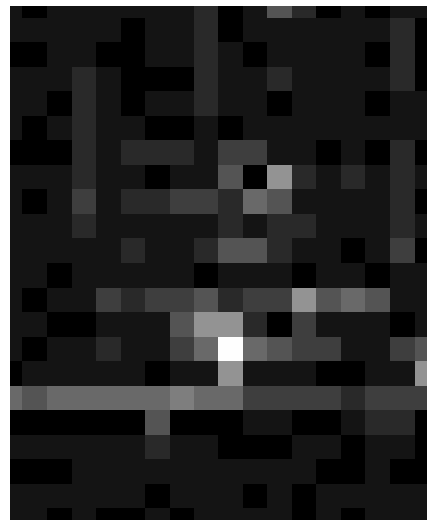

On-chip processing

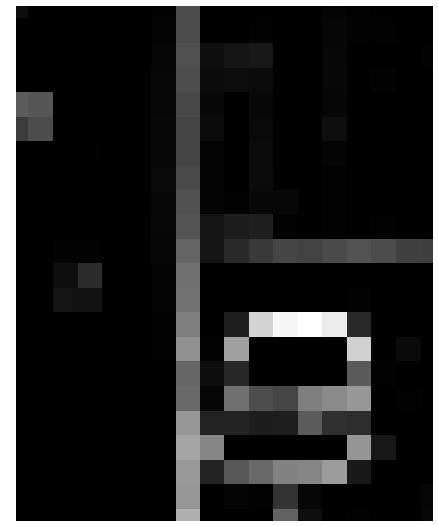

Ideal processing

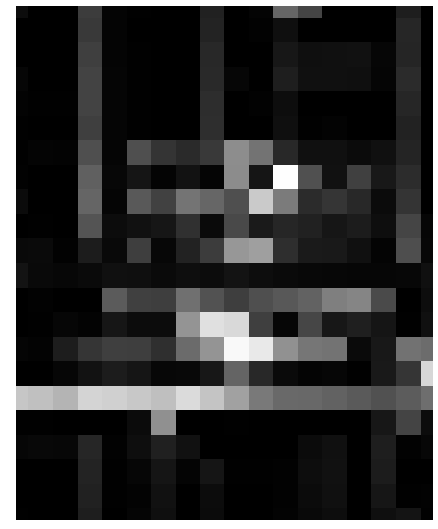

Ideal processing

FiguRE 5.23: Examples of energy-based scene representation.
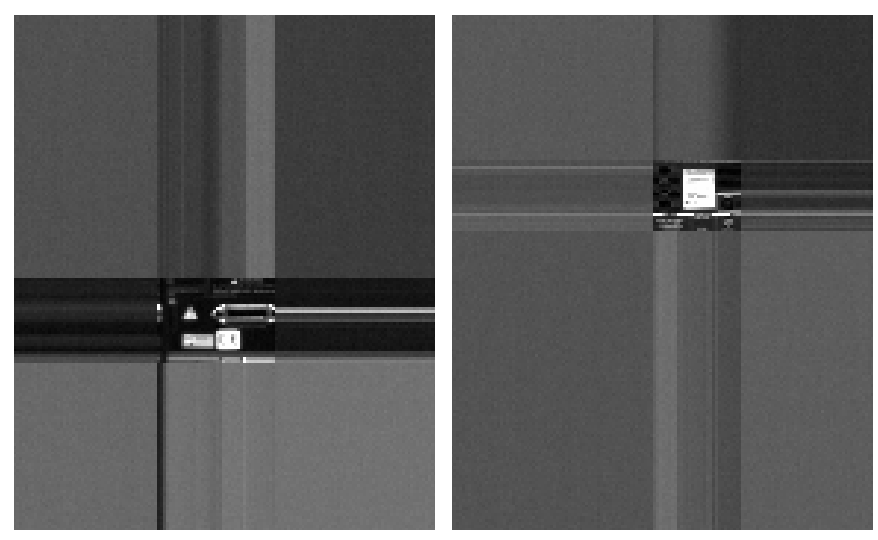

FiguRE 5.24: On-chip abrupt foveation around the blocks segmented by the computation of the energy in the second scene of Fig. 5.23. 


\begin{tabular}{cccccc}
\hline \hline $\begin{array}{c}\text { Block } \\
(\mathbf{p x})\end{array}$ & $\begin{array}{c}\text { VCO freq. } \\
(\mathbf{M H z})\end{array}$ & $\begin{array}{c}\text { Diffusion steps } \\
\left(N_{1}\right)\end{array}$ & $\begin{array}{c}\text { Energy } \\
\text { comput. }\end{array}$ & $\begin{array}{c}\text { Power consumption } \\
(\mathbf{m W})\end{array}$ & $\begin{array}{c}\text { Predicted power for } \\
\text { full-frame output }(\mathbf{m W})\end{array}$ \\
\hline \hline $4 \times 4$ & VCO off & External diffusion control & No & 2.7 & 14.7 \\
\hline $4 \times 4$ & 5 & 5 & No & 2.9 & 14.9 \\
\hline $4 \times 4$ & 50 & 5 & No & 3.5 & 15.5 \\
\hline $4 \times 4$ & 150 & 10 & No & 5.4 & 17.4 \\
\hline $8 \times 8$ & 150 & 10 & Yes & 5.6 & 17.6 \\
\hline $8 \times 8$ & VCO off & No diffusion & Yes & 2.9 & 14.9 \\
\hline $8 \times 8$ & VCO off & No diffusion & No & 2.0 & 14.0 \\
\hline
\end{tabular}

TABLE 5.3: Power consumption of the chip for different focal-plane configurations.

\subsection{Comparative analysis}

Several reported smart image sensors efficiently implement image filtering and multiresolution representation. The performance indexes chosen to establish a comparison are area and power consumption, together with image resolution and throughput. Minimizing area and power consumption has been the driving force for the design of the FLIP-Q prototype. Regarding the accuracy of the processing, no comparison can be made in general. In most of the cases the operation of the reported image sensors is accurate enough for the corresponding target application, but a thorough quantification of such accuracy is never given.

In [Kobayashi et al., 1991], Gaussian filtering with user-defined $\sigma$ is performed by means of a resistive network containing both positive and negative resistors. A very high power consumption is reported due mainly to the bias currents in the control circuit for the variable resistor. A simpler and more efficient implementation of this filtering is carried out in [Ni \& Guan, 2000]. In this case, a solver of the spatially-discretized diffusion process is implemented by means of a capacitive network. The variance of the filter is determined by a capacitor ratio, fixed by layout design, and an iteration number associated to the implicit time discretization of the network. The main argument given in favour of this implementation instead of another one based on a dynamic $\mathrm{RC}$ network is that usually the time constant of the latter is so small that sampling becomes difficult [Ni et al., 1993]. However, we have demonstrated with the FLIP-Q prototype that this problem can be overcome by a fine on-chip control. Better accuracy is thus achieved in spite of the intrinsic nonlinearities of the transistors while performing not discrete but continuous-time diffusion. Regarding the area and power consumption associated to the specific operation of Gaussian filtering, no data is given in [Ni \& Guan, 2000] to be compared with the performance of our prototype.

Vision chips capable of delivering programmable multiresolution scene representations have been also previously reported. In [Kemeny et al., 1997], capacitive networks outside the array are used to merge the pixel values. The main limitation of this chip is that its functionality is reduced to this operation. Besides, the blocks of pixels in which the image is divided must be square. 
The power consumption is of the same order of magnitude than that of the FLIP-Q prototype. The comparison in terms of area is more difficult to establish as the operation in [Kemeny et al., 1997] is not performed in-pixel but during the readout process. The die sizes, equalizing their resolutions by extrapolation, are very similar. Other processing arrays, like [Jaehyuk et al., 2007] and [Takahashi et al., 2009], use the multiresolution feature as a means to achieve a certain targeted outcome and therefore it is not separately characterized. In [Jaehyuk et al., 2007], the maximum possible reduction of resolution is by a factor of four outside the ROI while edge filtering at full-, half- and quarter resolution can be achieved in [Takahashi et al., 2009].

Table 5.4 summarizes the main reported features of the chips above commented. Although the functionalities of the different prototypes do not exactly match, we have tried to compute a figure of merit that contemplates their major features: $\mathrm{FOM}=($ Area $\cdot$ Power $) /($ Spatial resolution . Throughput). From these results, it can be seen that the FLIP-Q prototype, implementing image processing tasks which are useful for most of vision algorithms, presents very competitive figures, specially in terms of power consumption. Chips with lower FOM, [Kemeny et al., 1997] and [Jaehyuk et al., 2007], do not perform Gaussian filtering. Those which realize this type of filtering have similar [Ni \& Guan, 2000] or worse FOM [Kobayashi et al., 1991; Takahashi et al., 2009]. No chip delivering energy-based scene representations has been included in Table 5.4. To the best of our knowledge, this simplification of the scene at the focal plane had not been previously reported. Examples of other approaches for estimation of salient regions can be found in [Brajovic \& Kanade, 1998] and [Kwanho et al., 2009].

\subsection{Complementary information}

This section gathers information related to the package, pinage and programming of the prototype. The objective is to provide any potential user with all the necessary data to take advantage of the processing capabilities of the chip.

\subsubsection{Overview of the package}

The FLIP-Q prototype has been bonded into a ceramic 100-pin grid array package (Fig. 5.25) whose interconnection plan is depicted in Fig. 5.26. The bonding diagram is shown in Fig. 5.27. 


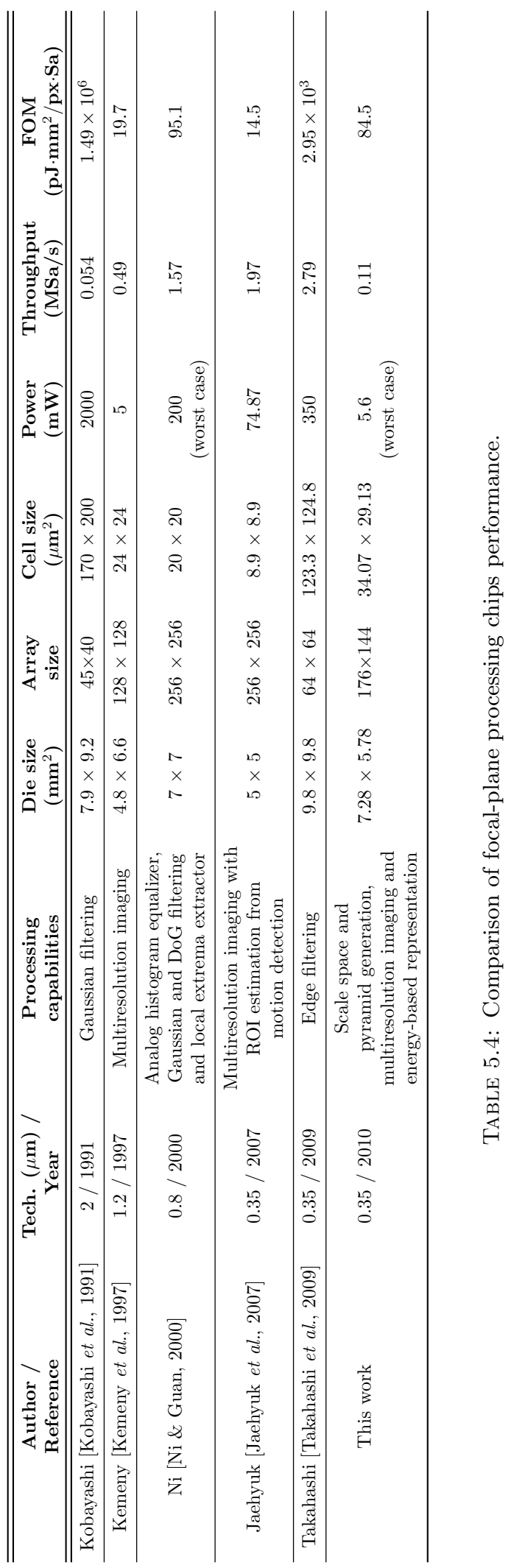



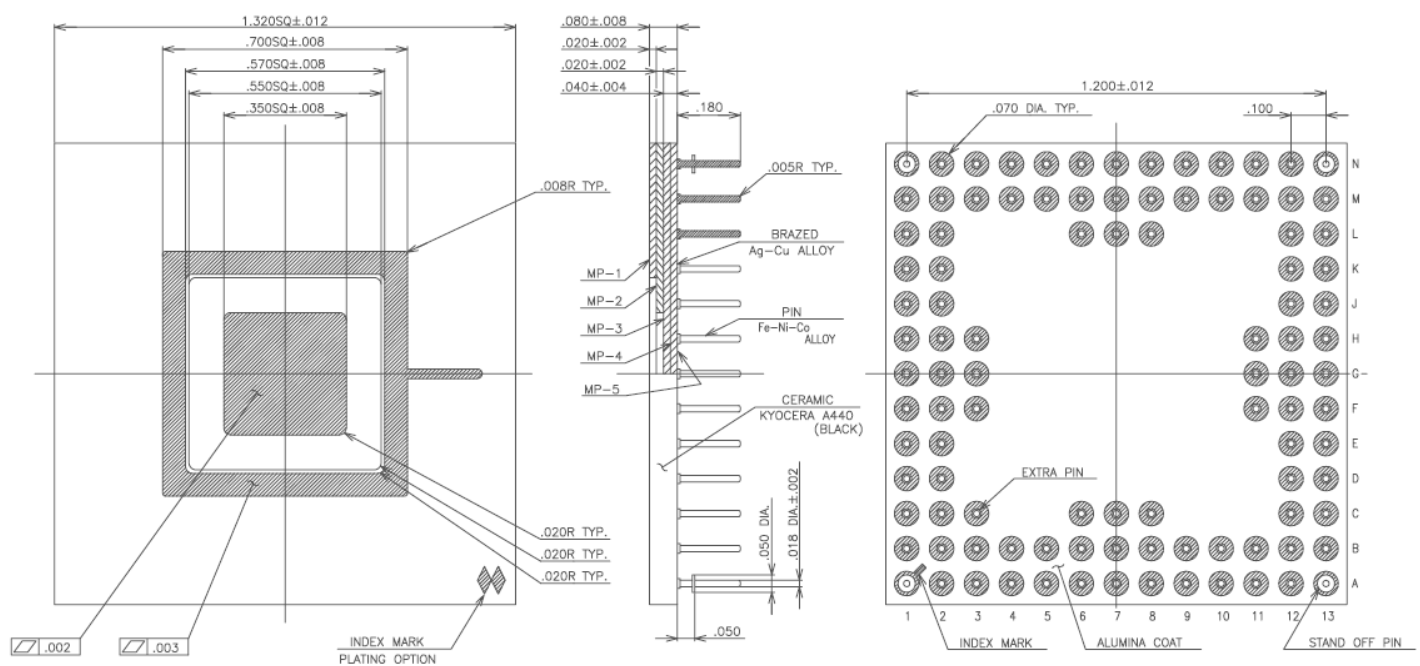

Figure 5.25: PGA-100 package

WIRE BOND PAD / CONNECTOR PIN INTERCONNECTION PLAN

\begin{tabular}{|c|c|}
\hline W/B NO. & PIN NO. \\
\hline 1 & B2 \\
\hline 2 & B1 \\
\hline 3 & C2 \\
\hline 4 & C1 \\
\hline 5 & D2 \\
\hline 6 & D1 \\
\hline 7 & E2 \\
\hline 8 & E1 \\
\hline 9 & F3 \\
\hline 10 & F2 \\
\hline 11 & F1 \\
\hline 12 & G2 \\
\hline 13 & G3 \\
\hline 14 & G1 \\
\hline 15 & H1 \\
\hline 16 & H2 \\
\hline 17 & H3 \\
\hline 18 & J1 \\
\hline 19 & J2 \\
\hline 20 & K1 \\
\hline
\end{tabular}

\begin{tabular}{|c|c|}
\hline W/B NO. & PIN NO. \\
\hline 21 & K2 \\
\hline 22 & L1 \\
\hline 23 & M1 \\
\hline 24 & L2 \\
\hline 25 & N1 \\
\hline 26 & M2 \\
\hline 27 & N2 \\
\hline 28 & M3 \\
\hline 29 & N3 \\
\hline 30 & M4 \\
\hline 31 & N4 \\
\hline 32 & M5 \\
\hline 33 & N5 \\
\hline 34 & L6 \\
\hline 35 & M6 \\
\hline 36 & N6 \\
\hline 37 & M7 \\
\hline 38 & L7 \\
\hline 39 & N7 \\
\hline 40 & N8 \\
\hline
\end{tabular}

\begin{tabular}{|c|c|}
\hline W/B NO. & PIN NO. \\
\hline 41 & M8 \\
\hline 42 & L8 \\
\hline 43 & N9 \\
\hline 44 & M9 \\
\hline 45 & N10 \\
\hline 46 & M10 \\
\hline 47 & N11 \\
\hline 48 & N12 \\
\hline 49 & M11 \\
\hline 50 & N13 \\
\hline 51 & M12 \\
\hline 52 & M13 \\
\hline 53 & L12 \\
\hline 54 & L13 \\
\hline 55 & K12 \\
\hline 56 & K13 \\
\hline 57 & J12 \\
\hline 58 & J13 \\
\hline 59 & H11 \\
\hline 60 & H12 \\
\hline
\end{tabular}

\begin{tabular}{|c|c|}
\hline W/B NO. & PIN NO. \\
\hline 61 & $\mathrm{H} 13$ \\
\hline 62 & $\mathrm{G} 12$ \\
\hline 63 & $\mathrm{G} 11$ \\
\hline 64 & $\mathrm{G} 13$ \\
\hline 65 & $\mathrm{~F} 13$ \\
\hline 66 & $\mathrm{~F} 12$ \\
\hline 67 & $\mathrm{~F} 11$ \\
\hline 68 & $\mathrm{E} 13$ \\
\hline 69 & $\mathrm{E} 12$ \\
\hline 70 & $\mathrm{D} 13$ \\
\hline 71 & $\mathrm{D} 12$ \\
\hline 72 & $\mathrm{C} 13$ \\
\hline 73 & $\mathrm{~B} 13$ \\
\hline 74 & $\mathrm{C} 12$ \\
\hline 75 & $\mathrm{~A} 13$ \\
\hline 76 & $\mathrm{~B} 12$ \\
\hline 77 & $\mathrm{~A} 12$ \\
\hline 78 & $\mathrm{~B} 11$ \\
\hline 79 & $\mathrm{~A} 11$ \\
\hline 80 & $\mathrm{~B} 10$ \\
\hline
\end{tabular}

\begin{tabular}{|c|c|}
\hline W/B NO. & PIN NO. \\
\hline 81 & $\mathrm{~A} 10$ \\
\hline 82 & $\mathrm{~B} 9$ \\
\hline 83 & $\mathrm{~A} 9$ \\
\hline 84 & $\mathrm{C} 8$ \\
\hline 85 & $\mathrm{~B} 8$ \\
\hline 86 & $\mathrm{~A} 8$ \\
\hline 87 & $\mathrm{~B} 7$ \\
\hline 88 & $\mathrm{C} 7$ \\
\hline 89 & $\mathrm{~A} 7$ \\
\hline 90 & $\mathrm{~A} 6$ \\
\hline 91 & $\mathrm{~B} 6$ \\
\hline 92 & $\mathrm{C} 6$ \\
\hline 93 & $\mathrm{~A} 5$ \\
\hline 94 & $\mathrm{~B} 5$ \\
\hline 95 & $\mathrm{~A} 4$ \\
\hline 96 & $\mathrm{~B} 4$ \\
\hline 97 & $\mathrm{~A} 3$ \\
\hline 98 & $\mathrm{~A} 2$ \\
\hline 99 & $\mathrm{~B} 3$ \\
\hline 100 & $\mathrm{~A} 1$ \\
\hline
\end{tabular}

\begin{tabular}{|c|c|}
\hline S/R & NC \\
\hline D/A & NC \\
\hline $\begin{array}{c}\text { EXTRA PIN } \\
\text { (C3) }\end{array}$ & NC \\
\hline
\end{tabular}

FiguRE 5.26: PGA-100 grid-cavity interconnection plan

\subsubsection{Pin description}

\subsubsection{Pad types}

Seven different pads have been employed in the chip, including power and ground pads, analog and digital inputs and outputs. Regarding the digital inputs, three different pads have been used. Two of them permit to set the default level of the corresponding signal, pulled-up for logic ' 1 ' and pulled-down for logic ' 0 '. The third one is intended for signals with special buffering requirements like for example clock inputs. Table 5.5 contains a short description of each pad. 


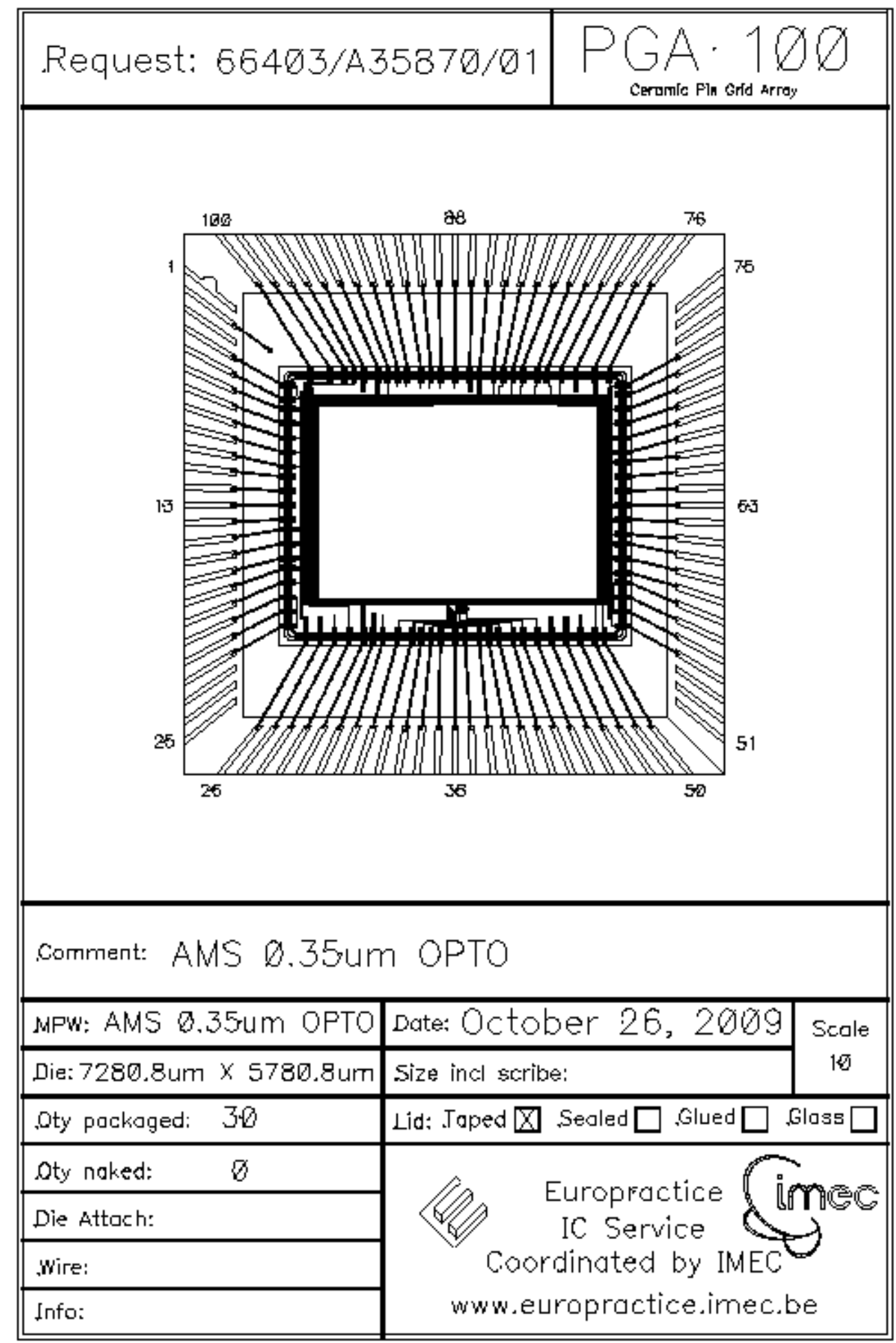

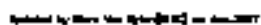

Figure 5.27: Bonding diagram of the chip for a ceramic 100-pin grid array. 


\begin{tabular}{|c|c|c|c|c|c|c|c|}
\hline Pad type & Signal type & Description & Volt. level & & $\begin{array}{l}\text { llled } \\
\text { down }\end{array}$ & Buffer & ESD \\
\hline VDD3ALLP & PWR & Power supply & $3.3 \pm 0.3$ & & & & \\
\hline GND3ALLP & GND & Ground connection & 0.0 & & & & \\
\hline ICDP & \multirow{3}{*}{ DIG_IN } & Digital input & {$[0.0,3.3]$} & & $\mathrm{X}$ & $\mathrm{X}$ & $\mathrm{X}$ \\
\hline ICUP & & Digital input & {$[0.0,3.3]$} & $\mathrm{X}$ & & $\mathrm{X}$ & $\mathrm{X}$ \\
\hline ICCK2P & & Clock input & {$[0.0,3.3]$} & & & $\mathrm{X}$ & $\mathrm{X}$ \\
\hline BU8P & DIG_OUT & Digital output (8mA drive) & {$[0.0,3.3]$} & & & $\mathrm{X}$ & $\mathrm{X}$ \\
\hline \multirow{2}{*}{ APRIO50P } & ANA_IN & Analog I/O & \multirow{2}{*}[0.0,3.3]{} & & & & X \\
\hline & ANA_OUT & $50 \Omega$ series resistance & & & & & \\
\hline
\end{tabular}

TABLE 5.5: Pad types employed in the prototype.

\subsubsection{Pin map}

The pin map, whose bottom view is depicted in Fig. 5.28 and top view in Fig. 5.29, is based on Table 5.5. It includes the name of signal assigned to every pin along with the corresponding pad type. In this way, the functionality and nature of a certain signal can be known at the same time. The label NC stands for "Not Connected" pin.

The 101 pins are thus grouped into:

- 12 power supply pins.

- 13 ground connections.

- 11 pins for 'vreset' (see Fig. 5.3).

- 8 analog inputs.

- 1 analog output.

- 29 digital inputs.

- 15 digital outputs.

- 12 pins without connection.

Tables 5.6-5.10 describe the pin assignment of the FLIP-Q prototype, complemented with some information about the characteristics of the signals. The definition of each column of the tables is:

- F. \#: Contains the number of the finger which the bonding wire is attached to.

- Pin \#: Displays the number (coordinates) of its corresponding pin.

- Pin name: Name of the signal assigned to the pin. 


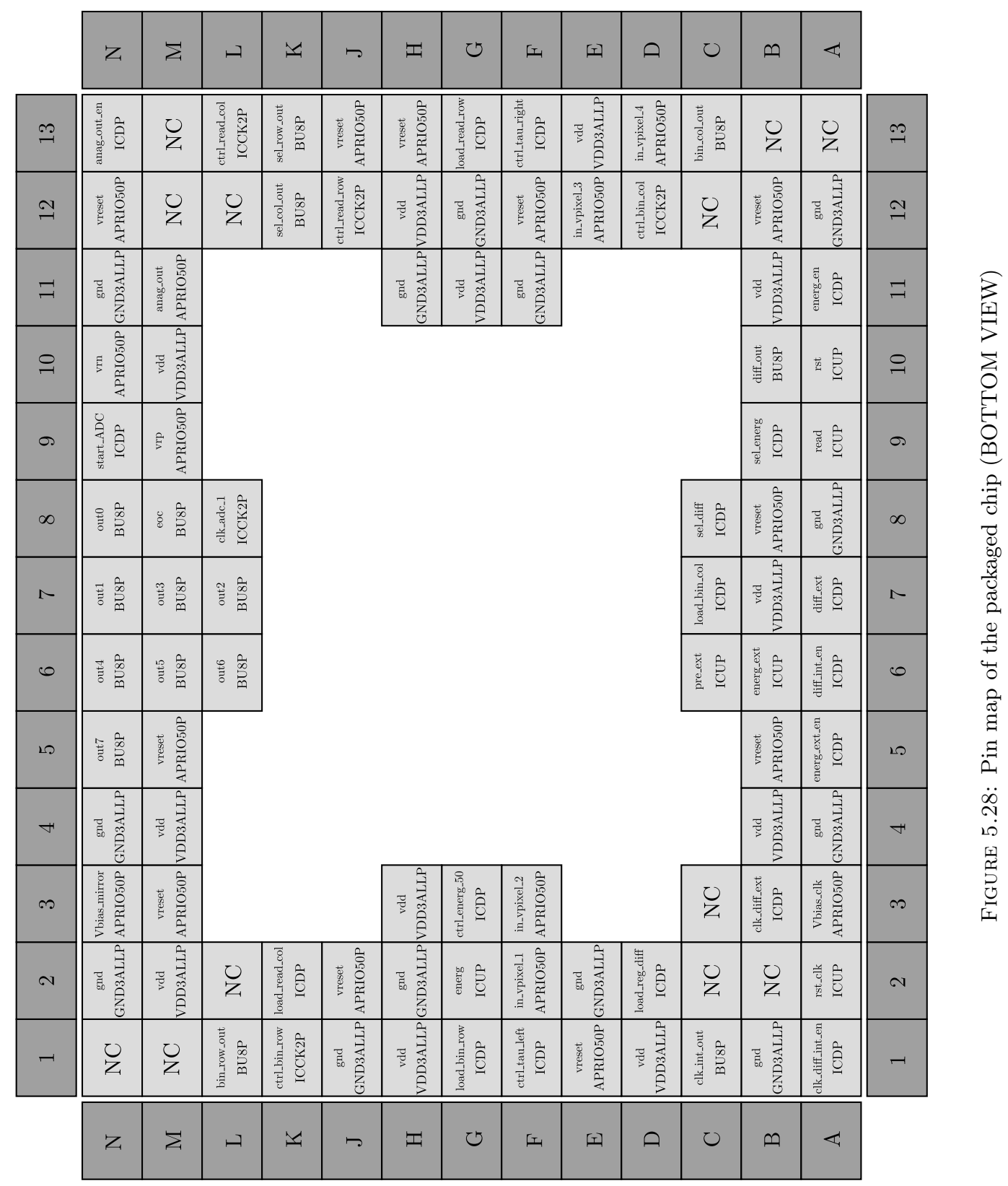




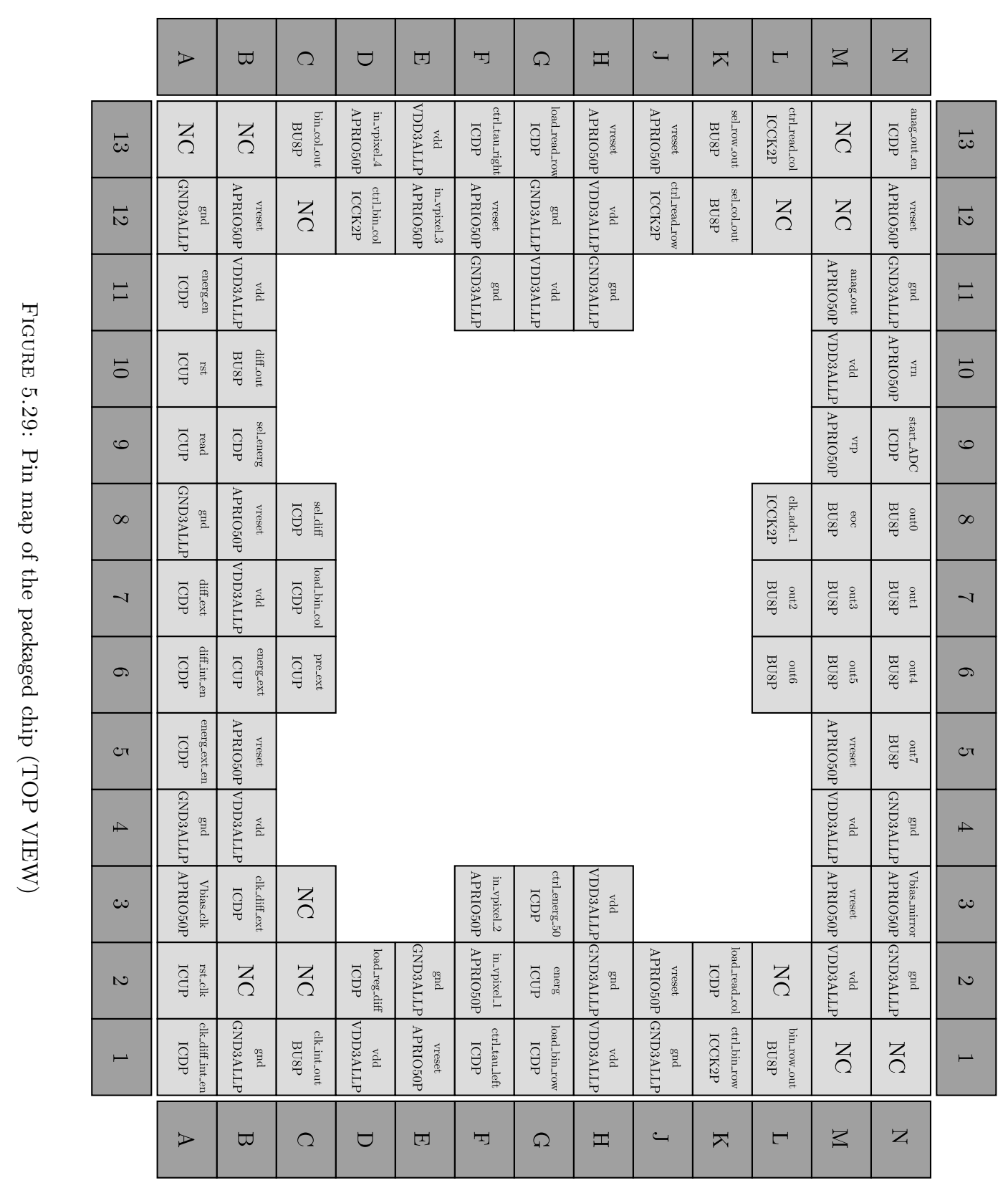


- Pad type: Indicates the pad type connected to the pin, according to Table 5.5.

- Signal type: Indicates the nature of the signal associated to the pin, according to Table 5.5.

- Description: Brief description of the signal. The active level of each digital signal is also provided (ActHI: active high, ActLO: active low).

- Levels: Voltage of the dc sources, range of the analog pads (minimum and maximum prescribed and/or expected) and voltages corresponding to the logic levels for the digital pads (H: 'high level'; L: 'low level'). 


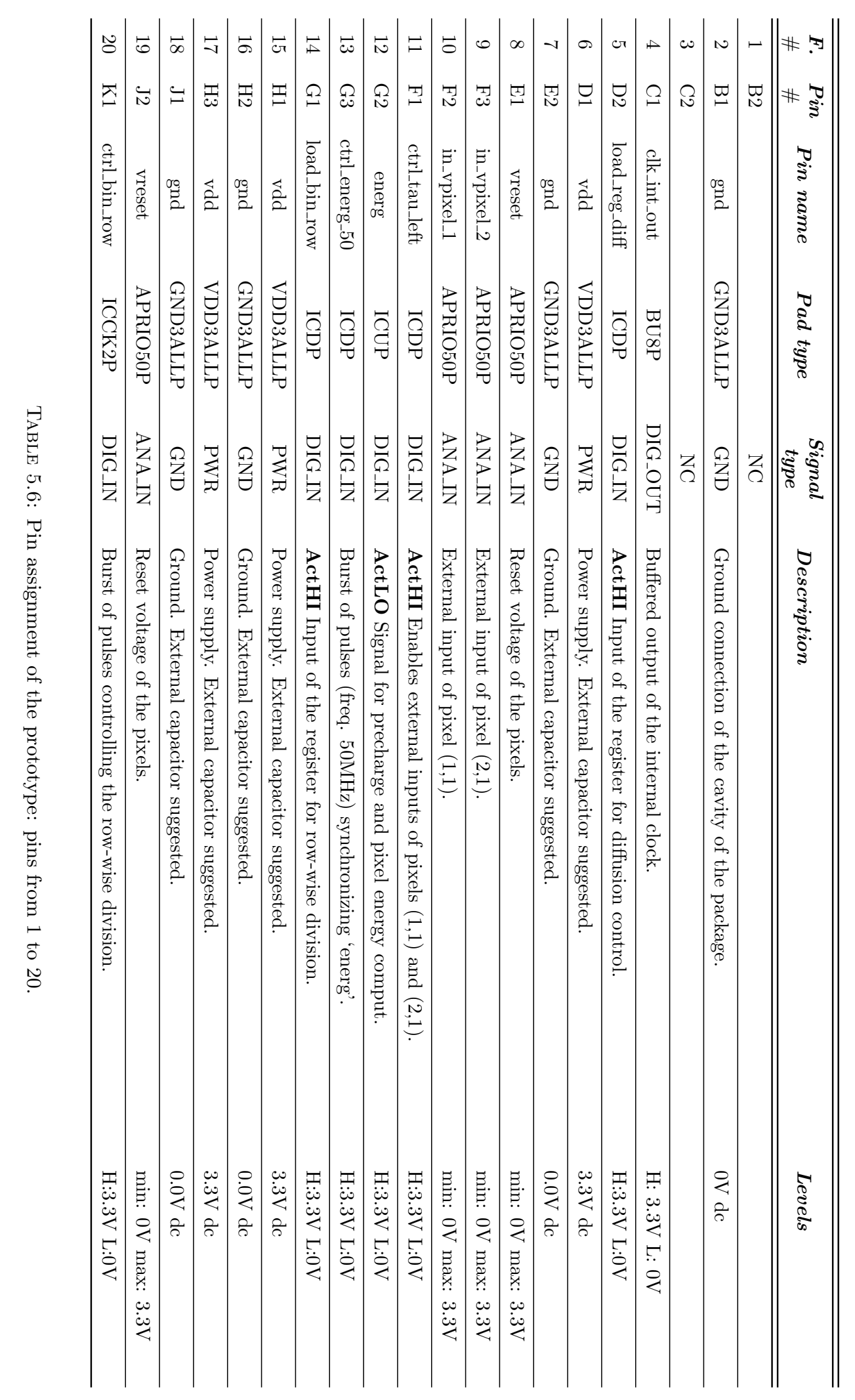




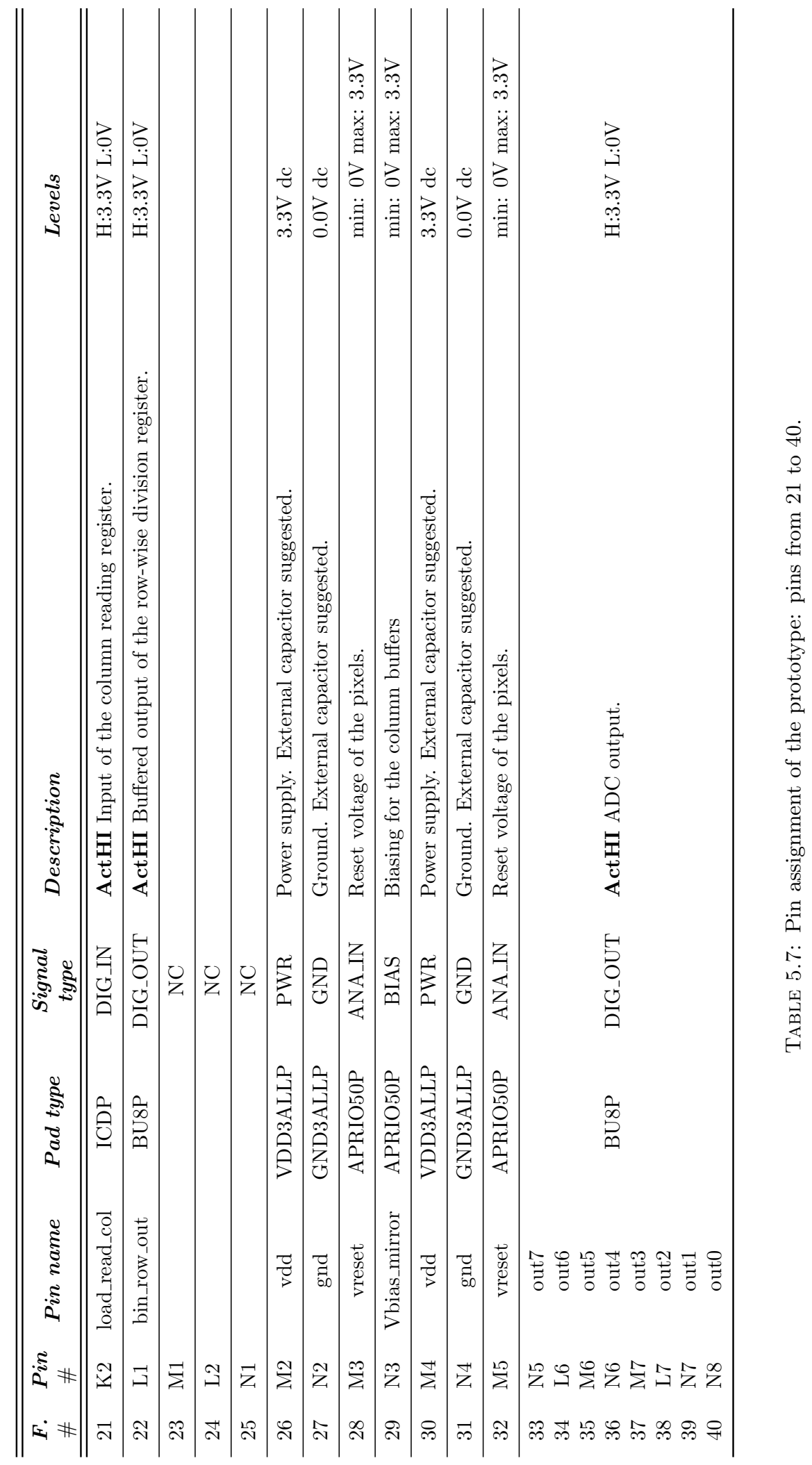




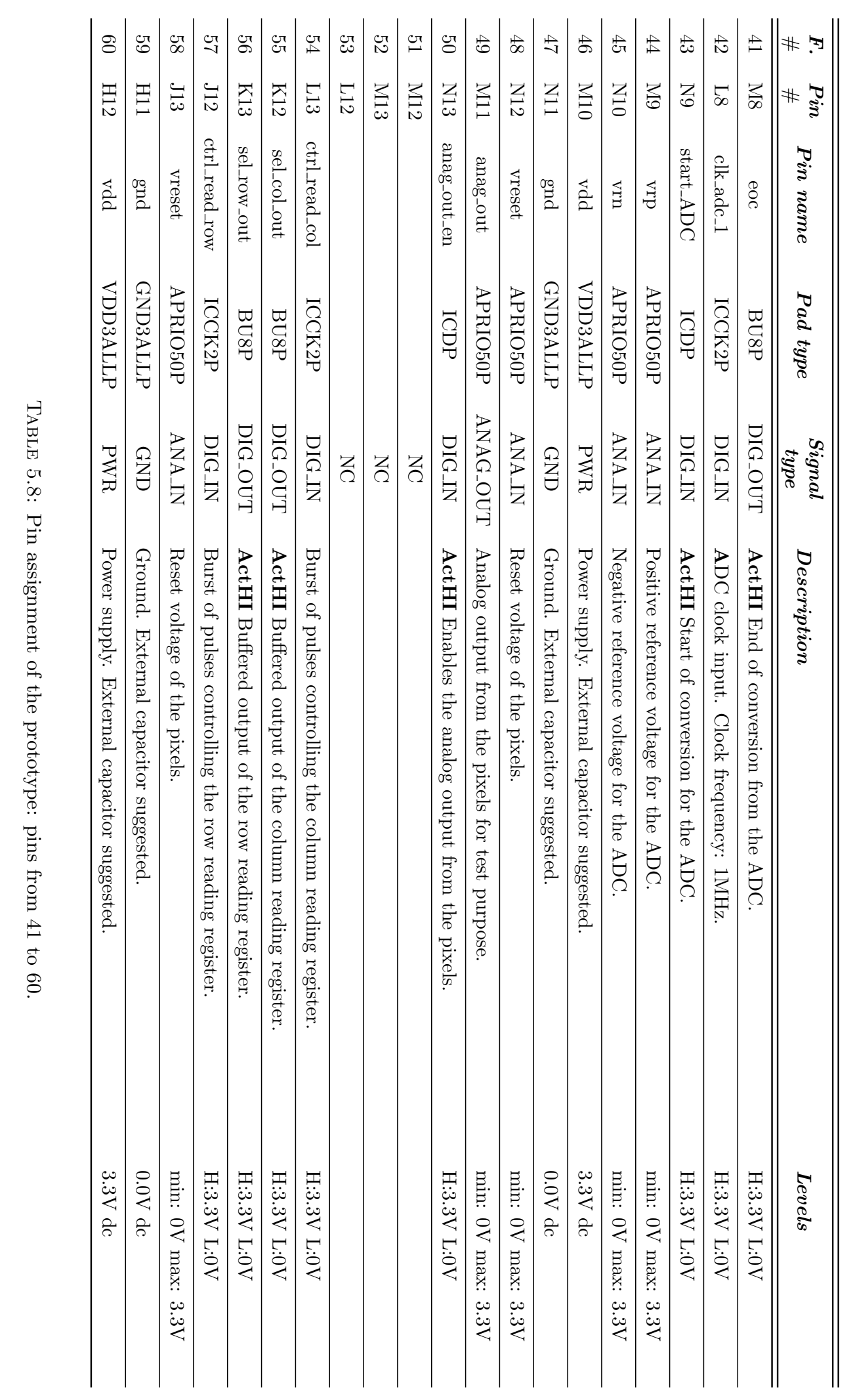




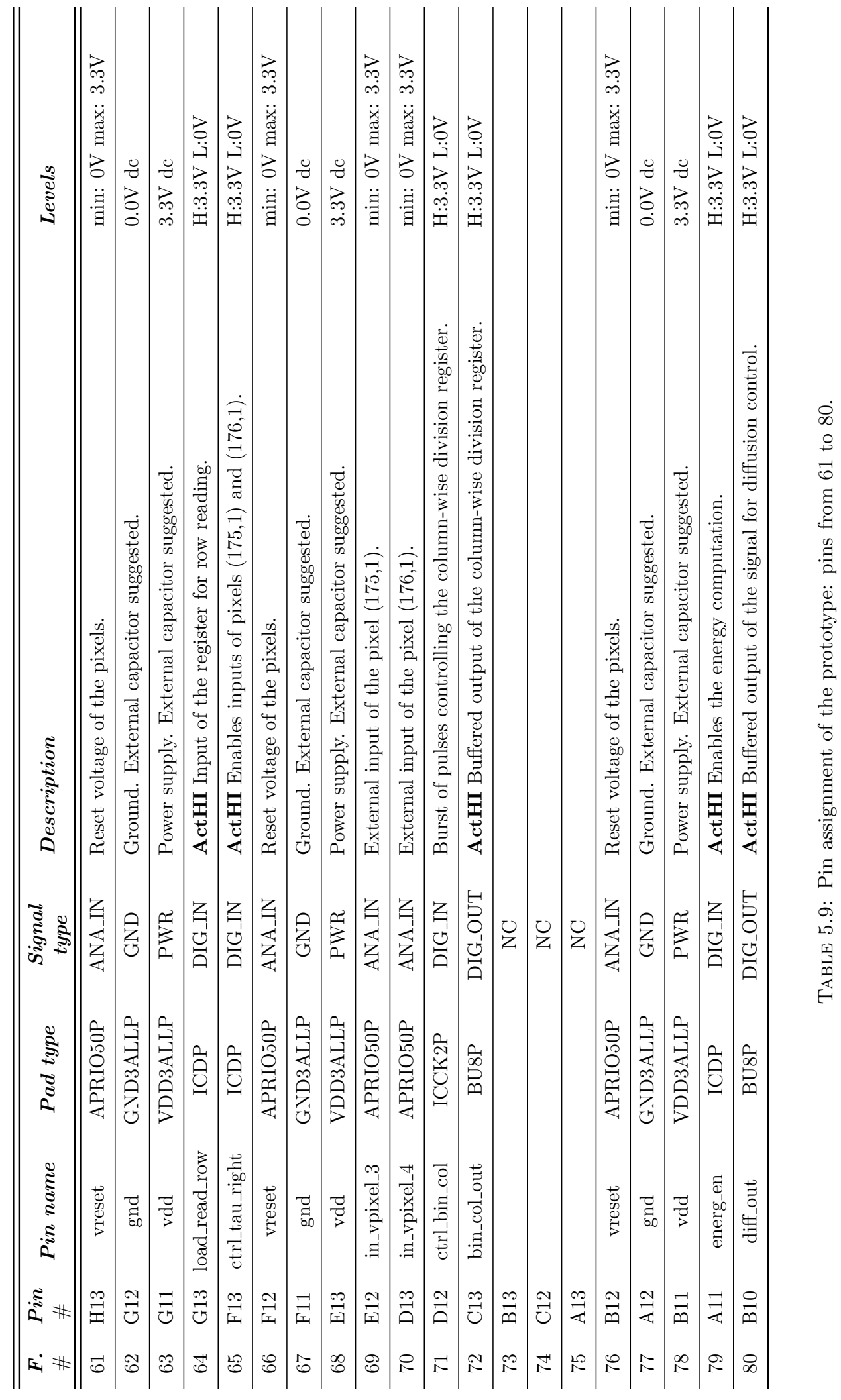




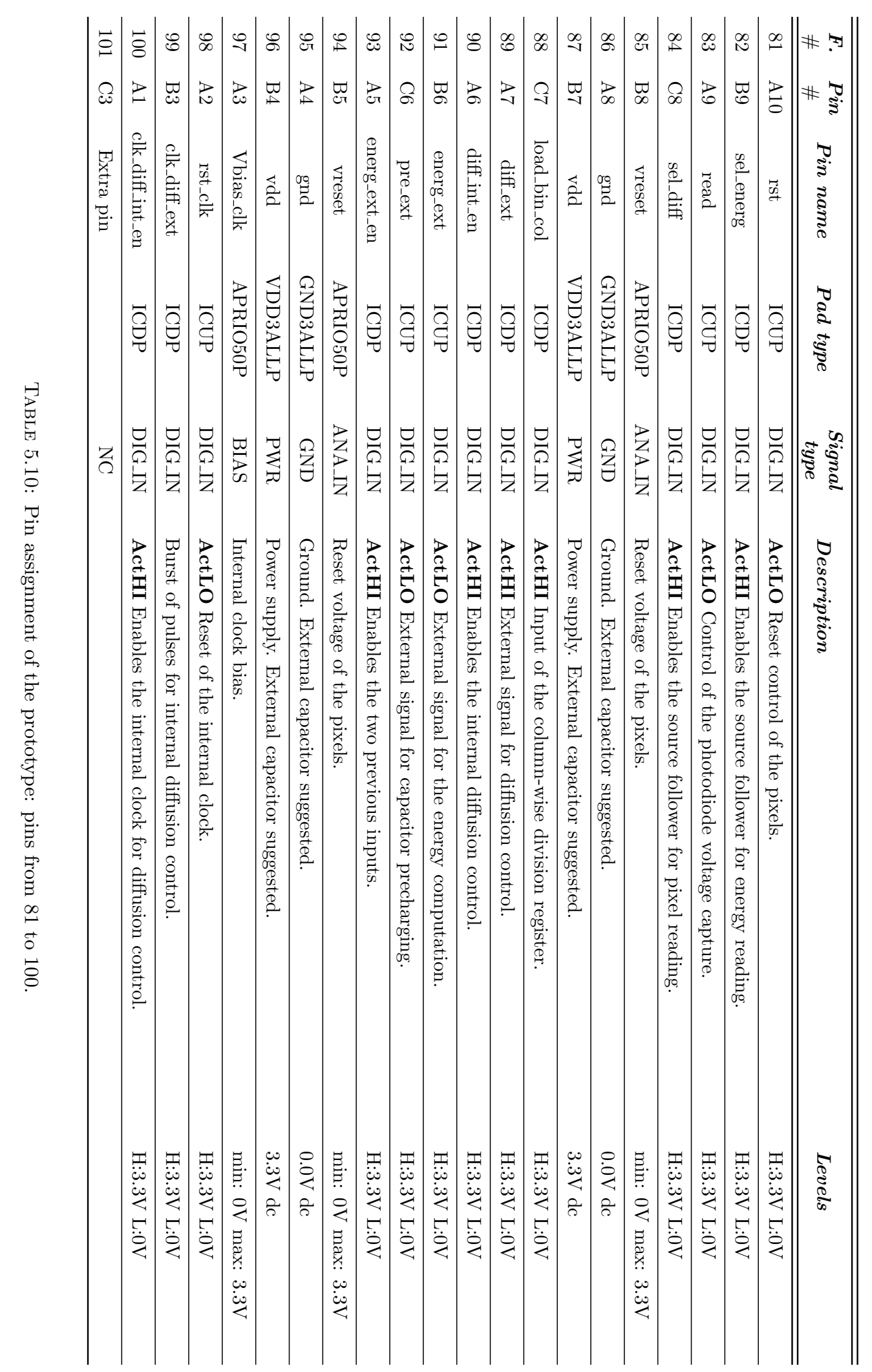




\subsubsection{Programming guide}

We report next how to configure the basic operation of the prototype and, specifically, how to carry out the processing primitives defined in Chapter 3.

\subsubsection{Basic operation}

\section{Bias setting}

To start with, power supply and ground pins must be set to $3.3 \mathrm{~V}$ and $0 \mathrm{~V}$ respectively. Then, 'vreset' pins must be set to $2.5 \mathrm{~V}$ for pixel global reset. The current biasing of the column buffers is realized by connecting a $10 \mathrm{k} \Omega$ resistor between the node 'Vbias_mirror' and power supply. Besides, if the internal clock is going to be used, it needs to be biased through the analog signal 'Vbias_clk'. This signal must be adjusted until reaching the targeted clock frequency, which can be monitored through 'clk_int_out'. The range of conversion for the A/D converter must be also established by means of 'vrn' - negative reference voltage - and 'vrp' - positive reference voltage. It is important to keep in mind that this range will be determined by the voltage to be read out $-V_{i j}$ or $V_{E_{i j}}$ - as well as by the transfer function of the source followers. Typical values are 'vrn' $=0.7 \mathrm{~V}$, 'vrp' $=1.55 \mathrm{~V}$ for $V_{i j}$ and 'vrn' $=1.2 \mathrm{~V}$, 'vrp' $=1.85 \mathrm{~V}$ for $V_{E_{i j}}$. Finally, 'in_vpixel_1', 'in_vpixel_2' at the upper left corner and 'in_vpixel_3', 'in_vpixel_4' at the upper right corner must be set to the adequate voltages for the calibration of $\tau$.

\section{Configuration of the focal-plane division}

In order to configure a prescribed block-wise focal-plane division, the column and row division control registers must be conveniently loaded. For example, a bit string of '1010...10' in both registers set all the blocks with a size of $2 \times 2$ px. Fig. 5.30 represents part of the loading process of such a bit string. Note that all the pixels will be completely isolated from their neighborhood by loading a bit string composed only of ' 0 's into both registers. On the contrary, bit strings composed only of ' 1 's will mean full 4-connection — 2- or 3-connection at the edges - of each pixel to its neighborhood.

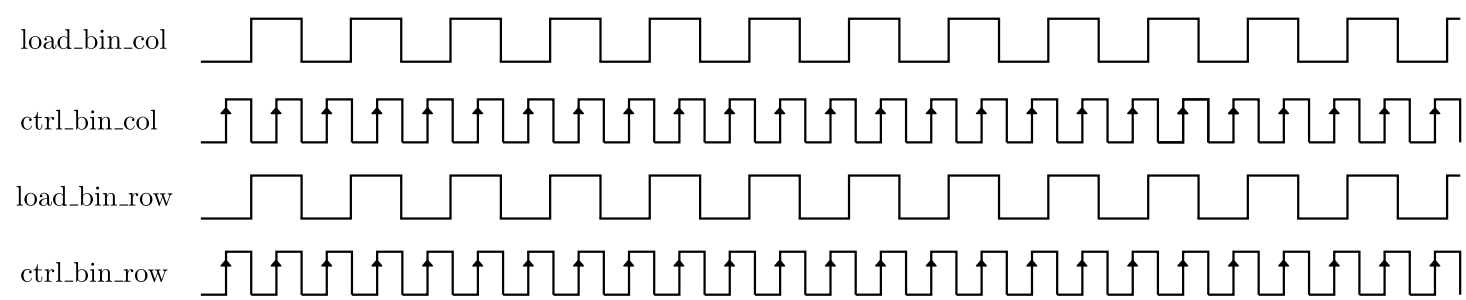

Figure 5.30: Loading process of the bit string '1010...10' at the focal-plane division registers. The pattern is repeated until loading all the bits into the registers. 


\section{Configuration of the reading process}

Both column and row reading registers must be loaded with all their bits to ' 0 ' but one of them set to ' 1 ', which determines the coordinates of the first pixel to be read out. An example is depicted in Fig. 5.31. Successive pixels will be selected by adequately shifting these ' 1 's. Bear in mind that the voltage $V_{i j}$ will be read out if 'sel_diff' is set to ' 1 ' and 'sel_energ' is set to ' 0 '. Alternatively, $V_{E_{i j}}$ will be read out if 'sel_diff' is set to ' 0 ' and 'sel_energ' is set to ' 1 '.

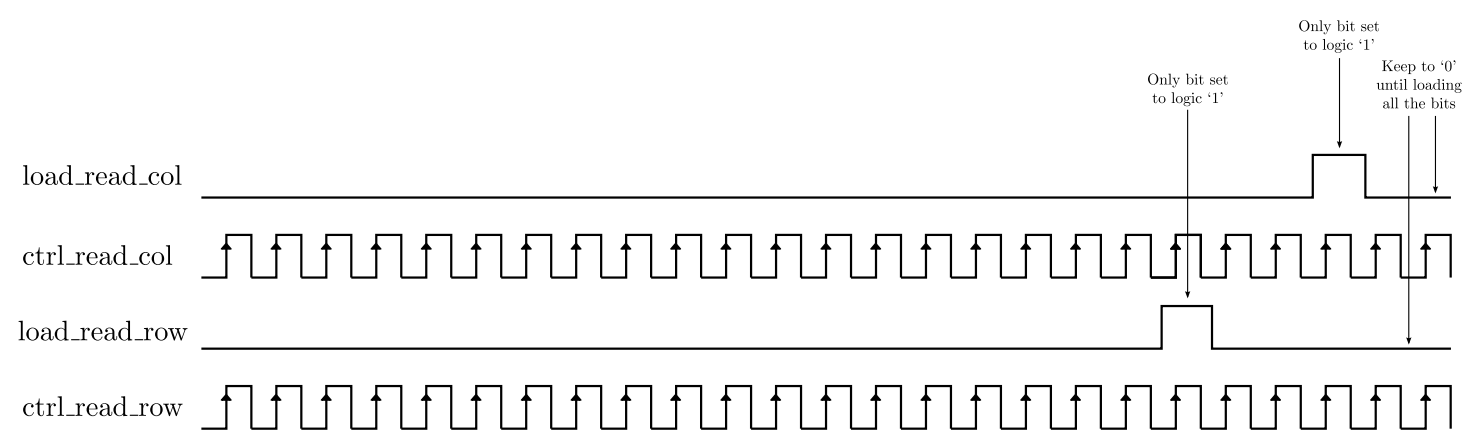

FiguRE 5.31: Example of a loading process of the reading registers.

\section{Frame capture}

Frames are captured through the signal 'rst' and 'read', which implement an electronic global shutter as depicted in Fig. 5.4.

\section{A/D conversion}

It is carried out according to the timing diagram in Fig. 5.32. The active-high signal 'start' begins the conversion. Then, the voltage $V_{i j}$ - or $V_{E_{i j}}$ - of the selected pixel is internally sampled and hold. The corresponding 8-bit digital representation, signaled by 'eoc', is available 9 clock cycles after sampling.

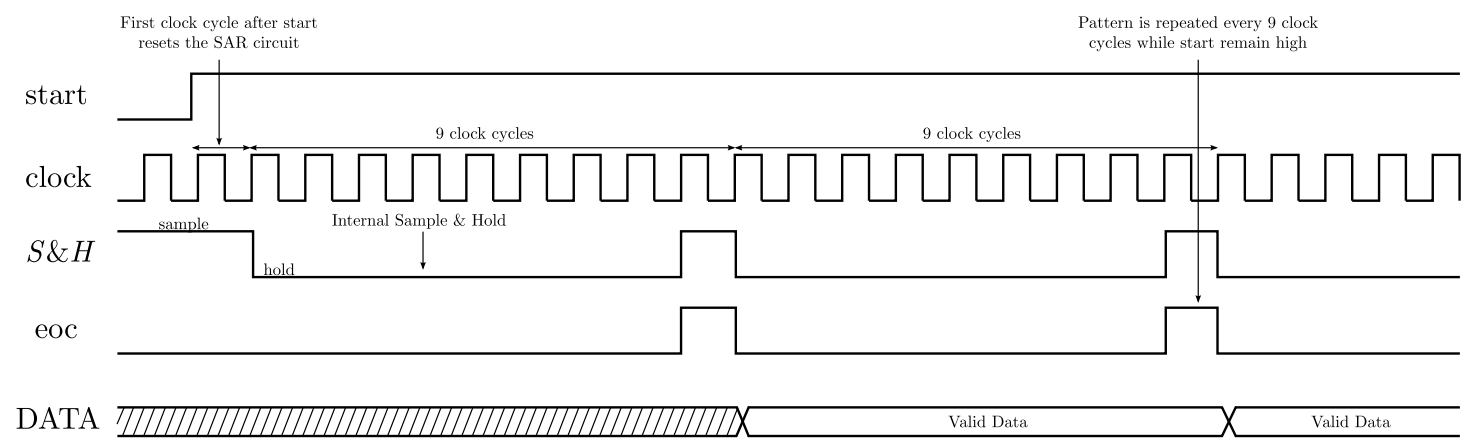

FiguRE 5.32: Timing diagram of the A/D converter operation. 


\subsubsection{Processing primitives}

The processing primitives implemented by the prototype are easily programmed once the basic operation of the chip has been established.

\section{Time-controlled linear diffusion}

The diffusion control register must be first loaded with as many '1's as necessary according to the diffusion interval $t$, as explained in Section 5.3.1. In Fig. 5.33, we show how this register is loaded with a bit string '110000000000'. Note that the register is externally clocked.

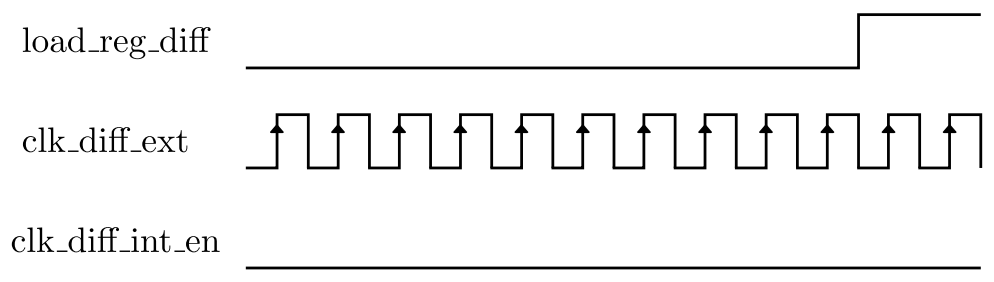

FiguRE 5.33: Loading the bit string '110000000000' at the diffusion control register with external clocking.

Then, assuming that the focal-plane division has been already configured, the only step left is to read out this bit string by using either the internal VCO (Fig. 5.34) or an external clock. The signal diff_ctrl will thus be active during the targeted time interval of diffusion $t$.

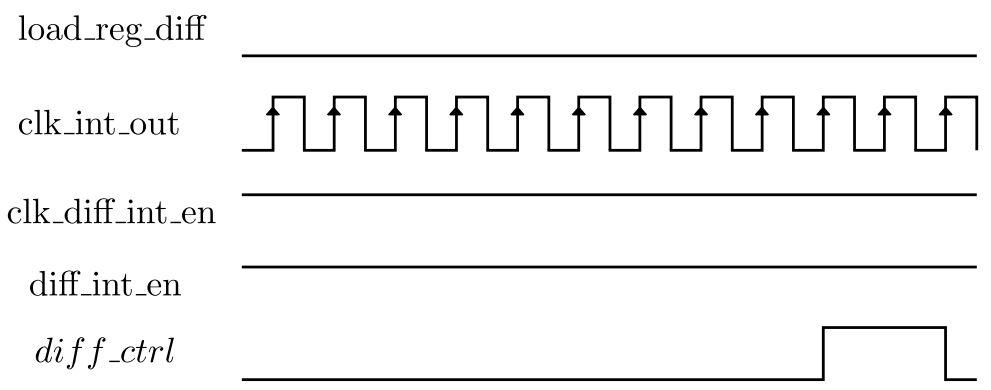

Figure 5.34: Reading out the bit string '110000000000' at the diffusion control register by using the internal VCO. 


\section{Multiresolution representation}

The targeted representation - half-resolution, quarter-resolution etc. — will depend on the focal-plane division configured. This representation will be ready to be read out just after finishing the frame capture. We must simply set 'diff_int_en' to ' 0 ' and 'diff_ext' to ' 1 ' before starting the global shutter. In this way, charge redistribution will take place concurrently with photosensing within each block.

\section{Image pre-distortion for reduced kernel filtering}

The steps to carry out this primitive are the same as for multiresolution representation. The key point is to deactivate charge redistribution by setting 'diff_ext' to ' 0 ' while the focal-plane division grid is shifted as described in Section 3.4. Once this shifting has been realized, 'diff_ext' must be set back to ' 1 ' to achieve the necessary averaging.

\section{Energy-based scene representation}

In order to compute the total energy associated to the blocks comprising a prescribed focalplane division, the first step is to set 'energ_en' to ' 1 '. Then, the internal signals $V_{\text {ctrl }}$ pre and $V_{c t r l_{E}}$ are generated as depicted in Fig. 5.35.

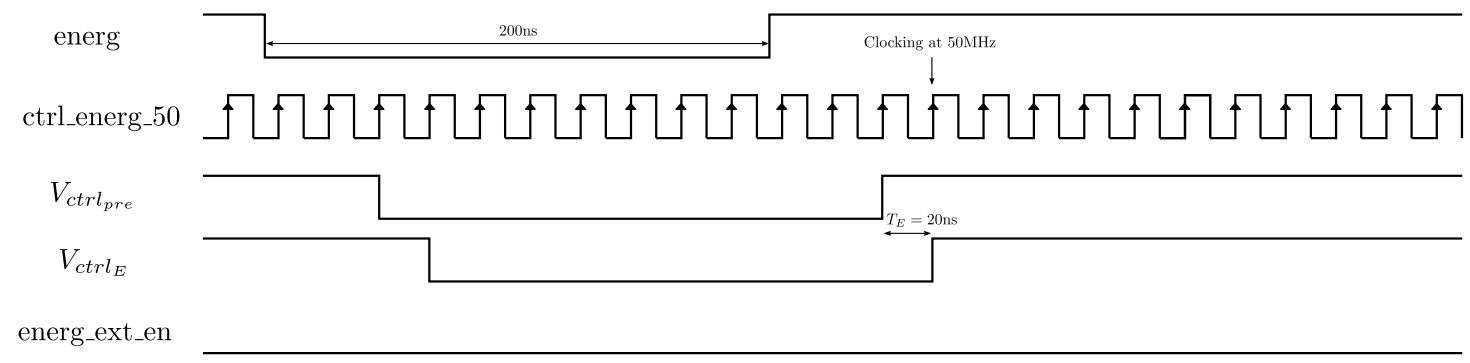

Figure 5.35: Generation of the signals for block-wise energy computation.

\subsection{Summary}

The FLIP-Q smart vision sensor has demonstrated the feasibility of an ultra low-power VLSI implementation of processing primitives capable of simplifying an image sequence to a userdefined extent. This sensor, due to its energy efficiency, is specially suitable to endow WSN nodes with vision capabilities. Thus, we will be addressing next the integration of the FLIP-Q prototype with a commercial WSN platform. As a measure of the efficiency claimed, we will see that the power consumption associated with this vision chip in the resulting system represents, in the worst case, only a $5.2 \%$ of the whole system power consumption. 


\section{Chapter 6}

\section{Wi-FLIP: A low-power vision-enabled WSN node}

Wi-FLIP is the system resulting from the integration of FLIP-Q, the prototype vision chip just described, and Imote2, the commercial WSN platform briefly commented in Section 2.1. This platform gathers certain features that make it appropriate for such integration. First of all, it contains a digital processor whose frequency can vary from $13 \mathrm{MHz}$ up to $416 \mathrm{MHz}$ with dynamic voltage scaling. This means an enormous flexibility when it comes to adjust the power consumption of the system in function of the timing requirements of the artificial vision application considered. Additionally, the amount of memory available, 256kB SRAM, 32MB SDRAM and 32MB FLASH, suffices for image processing algorithms of low-medium complexity, suitable for WSN environments. Finally, thanks to its dense pinout, Imote2 can carry out the control of FLIP-Q as well as retrieve the simplified scene representations it generates.

\subsection{Imote2: system description}

The top and bottom views of Imote2 (model IPR2400) [Imote2 (MEMSIC)] together with a block diagram containing its different modules are depicted in Fig. 6.1. The 32-bit ARM5 PXA271 XScale ${ }^{\circledR}$ processor [PXA271 (Marvell)] constitutes the system core. Its most remarkable feature is the above mentioned programmability of the clock frequency in conjunction with dynamic voltage scaling. A number of different low power modes is available. TinyOS [Levis \& Gay, 2009], an event-driven operating system tailored for low-power wireless devices, runs on this processor. An 802.15.4 radio [CC2420 (Texas Instr.)] with an on-board antenna is also integrated. The $\mathrm{CC} 2420$ radio transceiver supports a $250 \mathrm{kbps}$ data rate with 16 channels in the $2.4 \mathrm{GHz}$ band. The antenna enables a nominal range of about 30 meters. For longer ranges, 

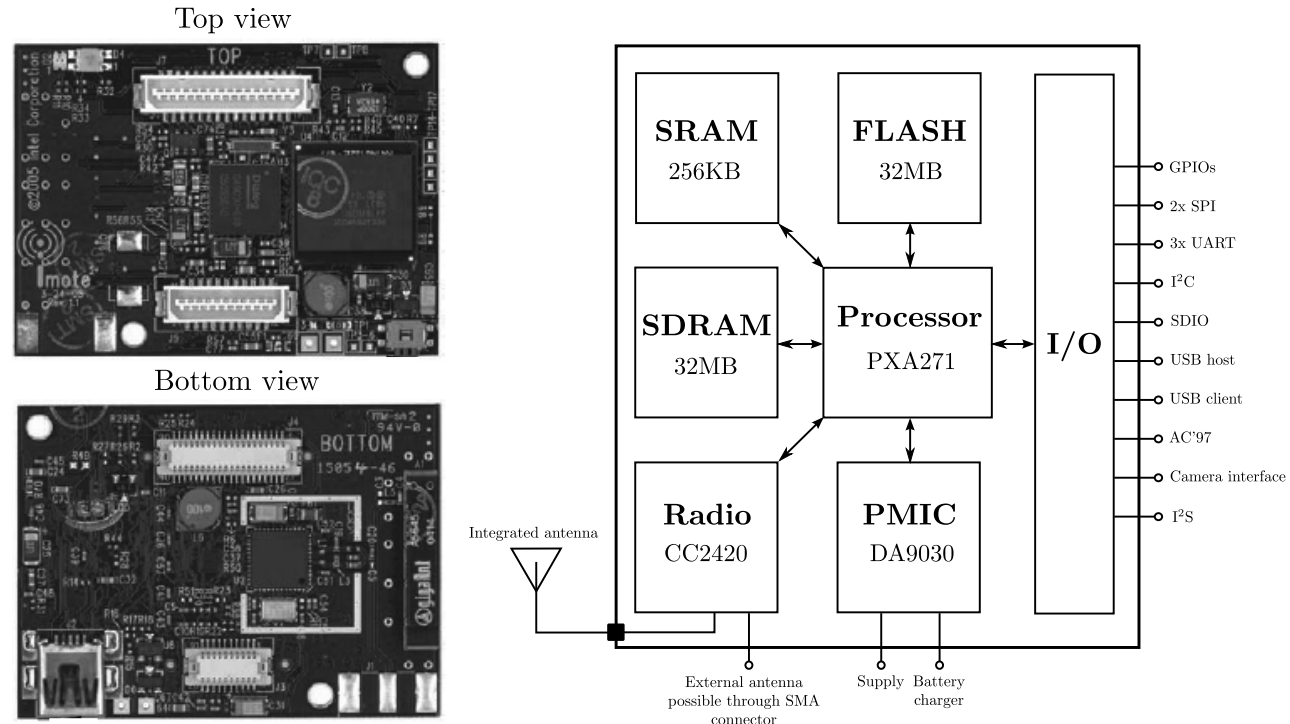

Figure 6.1: Top and bottom view of Imote2 along with its block diagram.

a SMA connector can be soldered directly to the board to connect an external antenna. Two interfaces for external interconnection are provided: a so-called "basic sensor board" interface, consisting of two connectors on the top side of the board, and an "advanced sensor board" interface, consisting of two high density connectors on the bottom side of the board. Through these interfaces, a wide set of standard I/O options is delivered. In our case, the most interesting aspect is the great number of GPIOs available. To supply the processor with all the required voltage domains, a Power Management Integrated Circuit (PMIC) is included. This PMIC supplies 9 voltage domains to the processor in addition to the dynamic voltage scaling capability. It also includes a battery charging option through USB and battery voltage monitoring. Imoted was designed to support primary and rechargeable batteries through an attachable battery board [IBB2400 (MEMSIC)], as well as to being powered via USB.

Table 6.1 summarizes the main operating parameters of Imote2 provided by the vendor. The figures related to power consumption have been confirmed during the experimental tests of Wi-FLIP. They correspond to a basic configuration where the minimum possible number of PXA271's modules are active. These figures are given in terms of current consumption, what results very useful to rapidly estimate the lifetime of the batteries. Note that, for the configuration where the PXA271 processor works at minimum clock speed, i.e. $13 \mathrm{MHz}$, the maximum current consumed by our prototype, $1.7 \mathrm{~mA}$, represents only the $5.2 \%$ of the whole system current consumption, $32.7 \mathrm{~mA}$. This percentage gets even lower as the clock speed increases. 


\begin{tabular}{|c|c|}
\hline \multicolumn{2}{|c|}{ Supply voltage } \\
\hline USB voltage & $5 \mathrm{~V}$ \\
\hline Battery voltage & $3.2 \mathrm{~V}-4.5 \mathrm{~V}$ \\
\hline \multicolumn{2}{|c|}{ Power consumption } \\
\hline Current draw in deep sleep mode & $390 \mu \mathrm{A}$ \\
\hline $\begin{array}{l}\text { Current draw in active mode } \\
\text { (clock speed: } 13 \mathrm{MHz} \text {, radio off) }\end{array}$ & $31 \mathrm{~mA}$ \\
\hline $\begin{array}{l}\text { Current draw in active mode } \\
\text { (clock speed: } 13 \mathrm{MHz} \text {, radio on) }\end{array}$ & $44 \mathrm{~mA}$ \\
\hline $\begin{array}{c}\text { Current draw in active mode } \\
\text { (clock speed: } 104 \mathrm{MHz}, \text { radio on) }\end{array}$ & $66 \mathrm{~mA}$ \\
\hline \multicolumn{2}{|l|}{ Radio } \\
\hline Frequency band (ISM) & $2400 \mathrm{MHz}-2483.5 \mathrm{MHz}$ \\
\hline Data rate & $250 \mathrm{kbps}$ \\
\hline Tx power & $-24 \mathrm{dBm}-0 \mathrm{dBm}$ \\
\hline Rx sensitivity & $-94 \mathrm{dBm}$ \\
\hline \multicolumn{2}{|c|}{ Mechanical } \\
\hline Board dimensions & $36 \mathrm{~mm} \times 48 \mathrm{~mm} \times 9 \mathrm{~mm}$ \\
\hline Weight & $12 \mathrm{~g}$ \\
\hline
\end{tabular}

TABLE 6.1: Summary of the main operating parameters of Imote2.

Bottom view

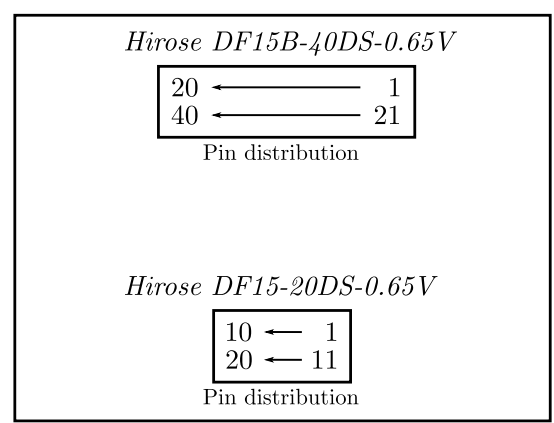

FiguRE 6.2: Connectors comprising the "advanced sensor board" interface of Imote2. 


\begin{tabular}{cccccc}
\hline \hline $\begin{array}{c}\text { Pin } \\
\text { \# }\end{array}$ & $\begin{array}{c}\text { PXA271's } \\
\text { GPIO \# }\end{array}$ & $\begin{array}{c}\boldsymbol{F L I P - Q} \\
\text { signal }\end{array}$ & $\begin{array}{c}\text { Pin } \\
\text { \# }\end{array}$ & $\begin{array}{c}\text { PXA271's } \\
\text { GPIO \# }\end{array}$ & $\begin{array}{c}\boldsymbol{F L I P - Q} \\
\text { signal }\end{array}$ \\
\hline \hline 1 & 82 & load_reg_diff & 21 & 81 & start_ADC \\
\hline 2 & 55 & ctrl_tau_left & 22 & 48 & ctrl_read_col \\
\hline 3 & 56 & energ & 23 & 50 & ctrl_read_row \\
\hline 4 & 57 & ctrl_bin_col & 24 & 51 & load_read_row \\
\hline 5 & 83 & load_bin_row & 25 & 52 & ctrl_tau_right \\
\hline 6 & 84 & ctrl_bin_row & 26 & MCLK & ctrl_energ_50 \\
\hline 7 & 85 & load_read_col & 27 & 54 & energ_en \\
\hline 8 & GROUND & GND & 28 & GROUND & GND \\
\hline 9 & USB - & & 29 & 106 & rst \\
\hline 10 & USB + & & 30 & 107 & sel_energ \\
\hline 11 & 28 & out7 & 31 & 12 & read \\
\hline 12 & 29 & out6 & 32 & PWM1 & clk_ADC \\
\hline 13 & 30 & out5 & 33 & GROUND & GND \\
\hline 14 & 31 & out4 & 34 & 10 & sel_diff \\
\hline 15 & 113 & out3 & 35 & 26 & load_bin_col \\
\hline 16 & GROUND & GND & 36 & 25 & diff_ext \\
\hline 17 & 98 & out2 & 37 & 24 & diff_int_en \\
\hline 18 & 100 & out1 & 38 & 23 & rst_clk \\
\hline 19 & 99 & out0 & 39 & 118 & clk_diff_ext \\
\hline 20 & 96 & eoc & 40 & 117 & clk_diff_int_en \\
\hline & & & & & \\
\hline
\end{tabular}

TABLE 6.2: FLIPQ-to-Imote2 interconnection plan.

\subsection{Interconnection plan}

The FLIPQ-to-Imote2 interconnection has been carefully realized according to the number of PXA271's GPIOs available. Specifically, there are 34 GPIOs which can be accessed through the 40-pin connector of the "advanced sensor board" interface depicted in Fig. 6.2. The interconnection plan is showed in Table 6.2. Only the strictly necessary logic to enable the processing primitives implemented by the $F L I P-Q$ sensor and retrieve the corresponding outcome is mapped into these GPIOs. Those signals included in the prototype for test purposes are dismissed. Notice that there are two FLIP-Q inputs that do not come from a GPIO. The first one is the signal 'ctrl_energ_50' (see Fig. 5.35), which can not be mapped into a GPIO due to its high frequency. Alternatively, it can be generated by means of an internal programmable frequency clock (MCLK) capable of reaching $52 \mathrm{MHz}$ when the PXA271 master clock is set to at least $208 \mathrm{MHz}$. The clock of the internal ADC does not come from a GPIO either. Instead, it is mapped into one of the four independent outputs generated by a PXA271's pulse width modulator (PWM). Image 
readout programming results thus much simpler. Finally, we take advantage of 4 connections to ground available at the 40-pin connector. The two pins left are useless for us as they can be only used as data signals for the USB client controller implemented in the PXA271 processor.

\subsection{Development board}

In order to carry out this interconnection plan and supply power and biasing to the FLIP$Q$ prototype, a 2-layer PCB has been designed and fabricated. Its schematic and layout are represented in Fig. 6.3. The ground reference is given by Imote2. Its distribution across the PCB is realized by taking advantage of the 4 pins above mentioned plus 2 additional ones from the 20-pin connector of the "advanced sensor board" interface. All the FLIP-Q GND pins are connected to this reference. The power supply is also provided by the mote through one of the 3 pins assigned to power external sensor boards at the 20-pin connector. This pin corresponds to the output voltage of an LDO (Low-DropOut) regulator belonging to the PMIC module. All the FLIP-Q PWR pins are thus connected to this regulator. Finally, in this first version of Wi-FLIP, potentiometers are used to adjust the biasing signals. In order to obtain enough accuracy, a voltage reference module is included. Its input voltage coincides with the supply voltage of Imote2 whereas its nominal output voltage is $3.3 \mathrm{~V}$. Two snapshots of the resulting vision-enabled WSN node are shown in Fig. 6.4.

\subsection{Wi-FLIP programming}

The first step to realize any experimental test with the $W i$-FLIP platform is to write the corresponding program, compile it and download it into the PXA271 processor. The standard programming language to develop applications running on TinyOS is nesC (network embedded system C) [Gay et al., 2003]. This language, a component-based C dialect, embodies the structuring concepts and execution model of TinyOS. One of its key points is a very efficient implementation of split-phase, or non-blocking, function calls. Thus, when one tell TinyOS to send, for example, a radio message, the corresponding 'send()' function returns almost immediately, well before the message is actually sent. At some point later, TinyOS calls a callback on the program telling it 'send()' completed. Split-phase code is often more verbose and complex than sequential code. But it has several advantages when it comes to networked devices with very little RAM. First of all, the system keeps responsive: there is never a situation when an application needs to take an action like, for instance, receiving a radio packet but all of its threads are tied up in blocking calls. Secondly, split-phase calls do not tie up stack memory while they are executing. And third, it tends to reduce stack utilization, as creating large variables on the 

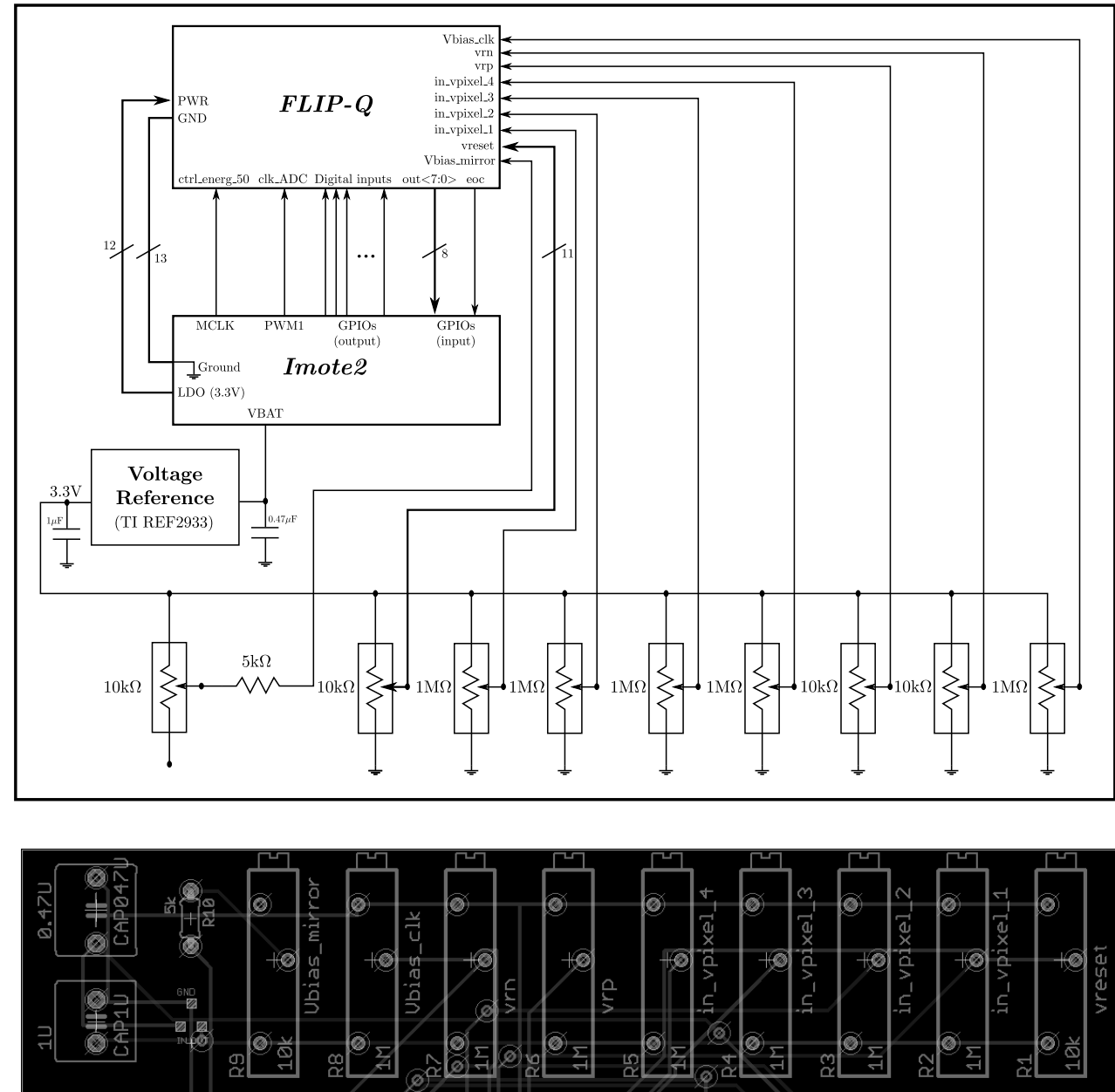

(3)
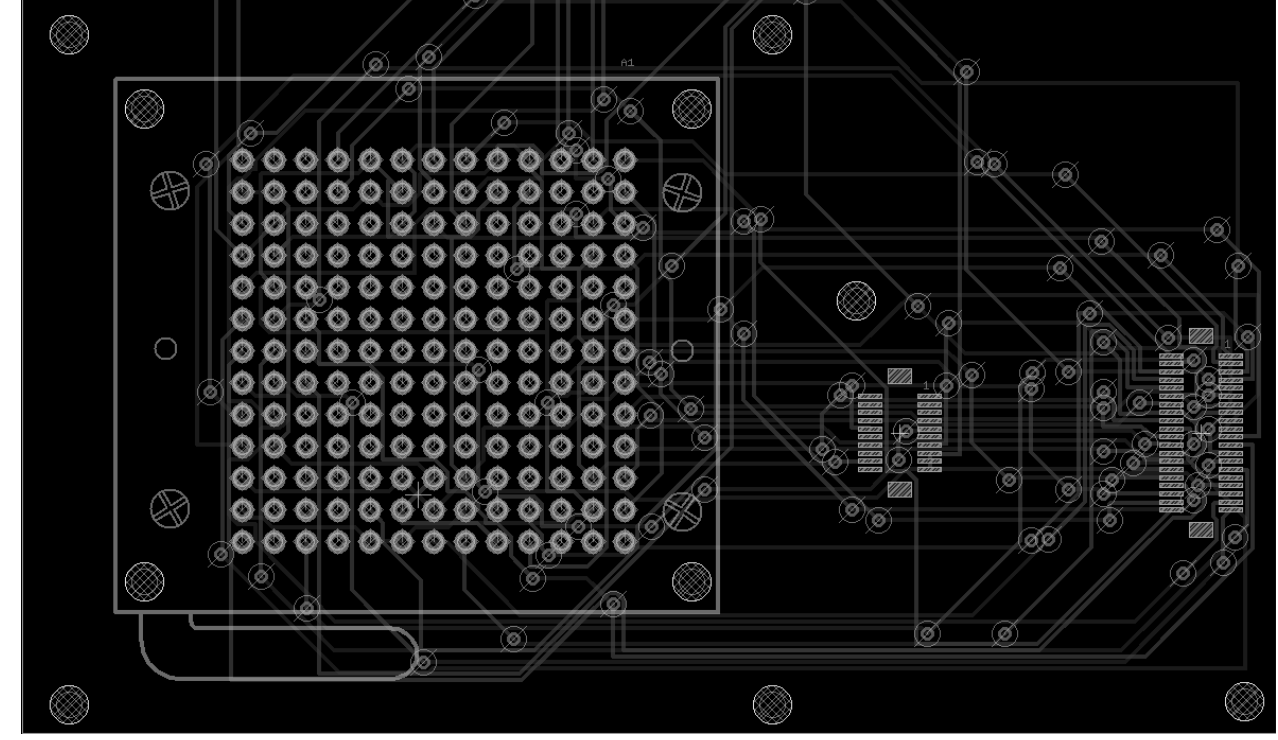

Figure 6.3: Wi-FLIP PCB: Schematic and layout. 

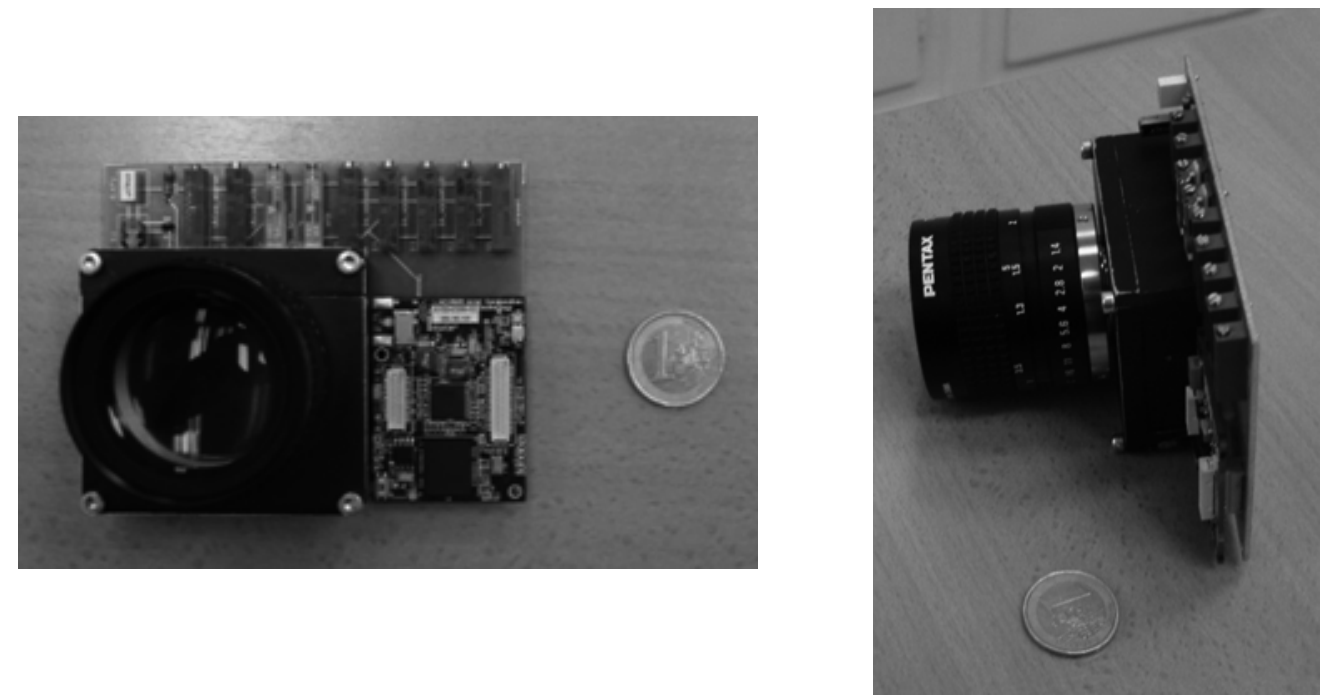

Figure 6.4: Wi-FLIP: a vision-enabled node for wireless applications.

stack is rarely necessary. Because nothing blocks, TinyOS expects no piece of code to run for a very long time. This is a critical issue because if a piece of code runs uninterrupted for a long time, other pieces of code can not run, and so the operating system might be forced to drop packets, miss timeouts, etc. This distinctive feature of TinyOS makes the power-efficient approach followed in this work to carry out low-level image processing tasks specially suitable. By having moved into the focal plane the implementation of such tasks, we are not only saving energy for the system as a whole but also making its programming and operation much simpler and efficient. Remarkably, we are avoiding the need of breaking the long and tedious computations intrinsic to these tasks into small parts in order to prevent the rest of the code from starving.

We have used the widely known cygwin environment to cross-compile nes $C$ code for the PXA271 processor. The resulting native code, ready to be executed, is downloaded into the mote via USB. All the applications developed follow the same previous steps before starting the execution of any algorithm:

1. Frequency change (if necessary): The clock speed of the processor is adjusted according to the timing requirements. The default value is $13 \mathrm{MHz}$. The rest of possibilities are $104 \mathrm{MHz}$, $156 \mathrm{MHz}, 208 \mathrm{MHz}, 312 \mathrm{MHz}$ and $416 \mathrm{MHz}$.

2. Voltage level adjustment: The high level of all the pins associated with signals controlling the operation of FLIP-Q must be set to $3.3 \mathrm{~V}$. This is a mandatory step as the default value is $2.7 \mathrm{~V}$.

3. Configure pins: The GPIO pins must be configured as input or output depending on the signal mapped into each one. 
4. Configure timers: Two timers must be at least configured in order to implement the electronic global shuttering for FLIP-Q.

5. Configure and activate the required PXA271's modules: These modules are the PWM for the FLIP-Q's ADC clock, the internal clock MCLK if the energy-based scene representation is to be obtained and an UART and/or the radio if the results are going to be output (see below).

Keep in mind that the biasing signals must be also manually adjusted through the potentiometers before executing any code.

Regarding the outcome of $W i-F L I P$, we have implemented two different ways of retrieving it for further analysis. The first option is through the commercially available Imote2 Interface Board [IIB2400 (MEMSIC)] (Fig. 6.5(a)). This board, which can be stacked onto Imote2 thanks to its compatible set of connectors, maps a USB port into 2 serial ports corresponding to 2 PXA271's UARTs. We can thus send out a stream of bytes comprising the result of the processing by using any of these UARTs. Subsequently, the Interface Board will put the stream into a USB connection to, in our case, a PC running MATLAB ${ }^{\circledR}$. The second option, slower, is via radio. In this case, we need a base station like that of Fig. 6.5(b) [MIB520 (MEMSIC)]. This base station picks up the radio signal coming from Wi-FLIP and maps it into a USB connection to, again, a PC running MATLAB ${ }^{\circledR}$. The key point now is to tune the same radio channel on which $W i$-FLIP is transmitting in the base station. Additionally, the format of the information sent by Wi-FLIP must be known a priori by the base station in order to adequately extract it from the radio packages. We have also used cygwin to cross-compile nes $C$ code for the base station taking into account all these aspects.

\subsection{Experimental tests ${ }^{1}$}

\subsubsection{FLIP- $Q$ processing primitives}

The first tests programmed in Wi-FLIP have consisted of capturing a frame, applying one of the different primitives implemented by the smart vision sensor and outputting the resulting image. We have thus corroborated the functionality of the prototype in a totally different test environment to that of Fig. 5.12. Examples of scale space generation, multiresolution representation and energy-based salient region estimation are depicted in Figs. 6.6, 6.7 and 6.8, respectively. For the last case, we proceed exactly like in Section 5.5.6 but now the focal plane is divided into

\footnotetext{
1 (C)2011 IEEE. Reprinted, with permission, from J. Fernández-Berni et al. "Wi-FLIP: A Wireless Smart Camera Based on a Focal-plane Low-power Image Processor", Fifth ACM/IEEE International Conference on Distributed Smart Cameras, pp. 1-4, Ghent, Belgium, August 22-25, 2011.
} 


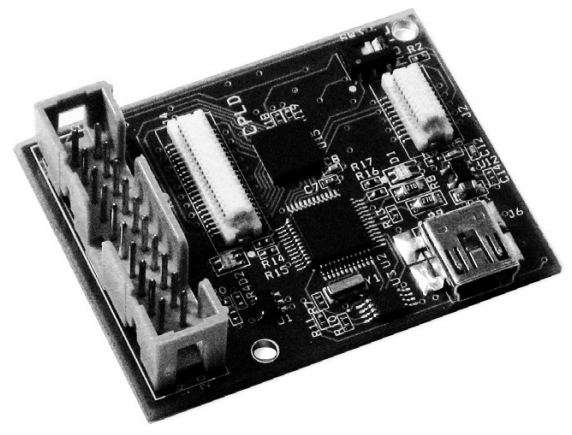

(a)

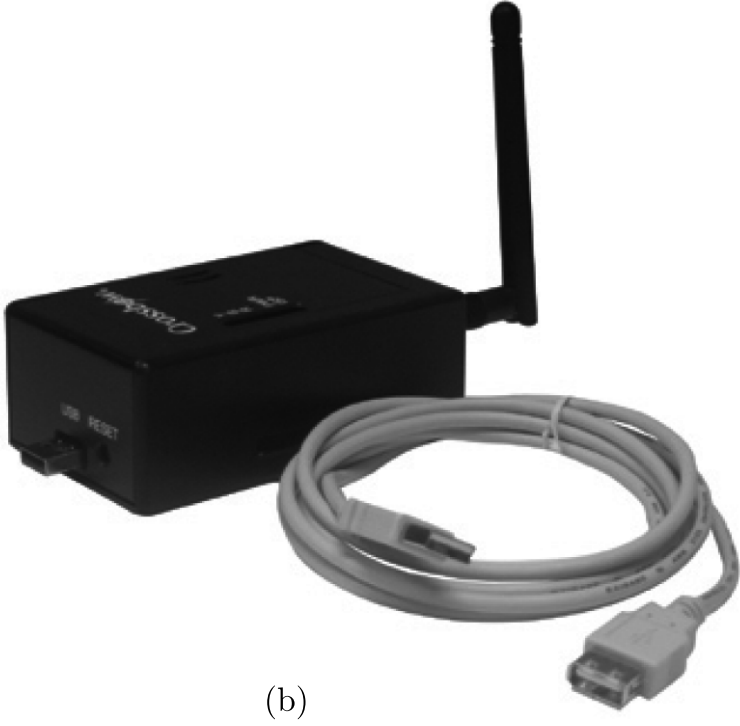

(b)

Figure 6.5: Imote2 Interface Board IIB2400 (a) and base station MIB520 (b): two different ways of retrieving information from $W i-F L I P$.

blocks of $4 \times 4 \mathrm{px}$ instead of $8 \times 8 \mathrm{px}$. The computation of the energy difference at each block is realized by the PXA271 processor.
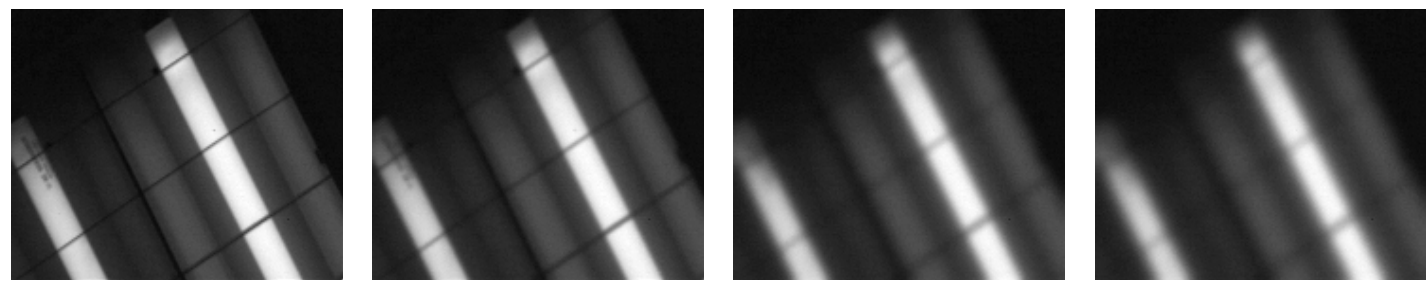

Figure 6.6: Scale space generation in Wi-FLIP. The diffusion is internally controlled as explained in Section 5.3.1.

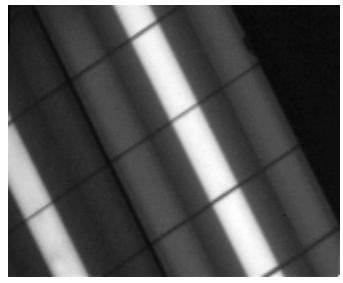

Original image

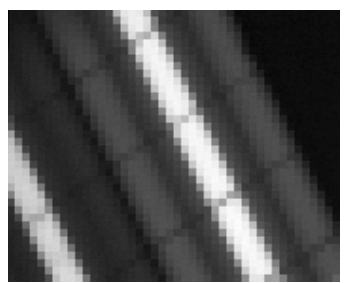

Focal-plane blocks of $4 \times 4 \mathrm{px}$

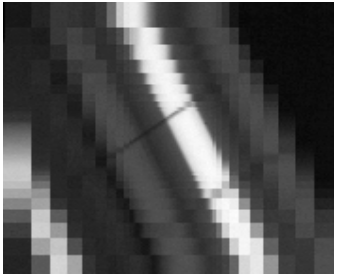

Progressive focal-plane division

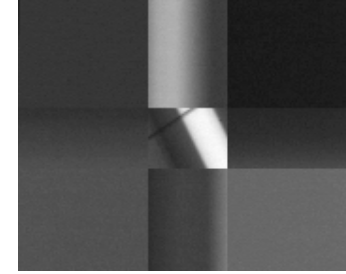

Abrupt focal-plane division

FiguRE 6.7: Example of multiresolution representation in Wi-FLIP.

\subsubsection{Exposure time control algorithm}

Once the basic operation of Wi-FLIP was checked out, we addressed the programming of more elaborated tasks commonly needed for vision-based applications. As a first approach to such 

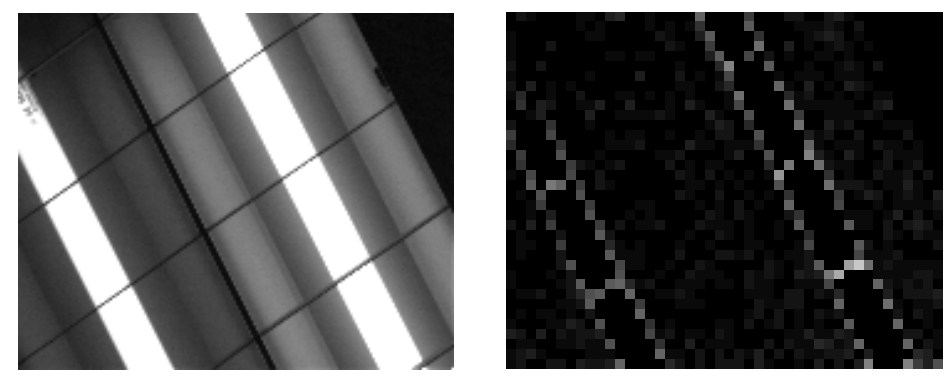

Figure 6.8: Salient region estimation in $W i-F L I P$.

tasks, we have implemented an algorithm which adapts the exposure time, $T_{\text {exp }}$, to the characteristics of the scene at the moment. Operating in photocurrent integration mode, the voltage $V_{i j}$ representing the value of each pixel depends on $T_{\text {exp }}$. Thus, for the same power of incident light over the sensor surface, a larger or smaller value of $T_{\text {exp }}$ will result respectively in a larger or smaller excursion of $V_{i j}$ from the reset voltage. If $T_{e x p}$ is not correctly set, we will obtain too dark or too bright images. A simple mechanism to adjust $T_{\text {exp }}$ is to force that the mean value of the image, namely:

$$
\bar{V}=\frac{1}{M N} \sum_{i=1}^{M} \sum_{j=1}^{N} V_{i j}
$$

falls around the middle point of the nominal pixel voltage range, $V_{\text {mid }}$. In this way, we make sure that most of the pixels are neither over-exposed nor under-exposed according to the current conditions of the scene. Let us define $\bar{T}_{\text {exp }}$ as the exposure time achieving this.

The algorithm works with five prescribed parameters, namely: $T_{\exp _{M I N}}$ and $T_{\exp _{M A X}}$ defining the range of possible exposure times, i. e. $\bar{T}_{\exp } \in\left[T_{\exp _{M I N}}, T_{\exp _{M A X}}\right], V_{L}$ and $V_{H}$ defining the interval which must contain $\bar{V}$, that is $\bar{V} \in\left[V_{L}, V_{H}\right]$ with $V_{\text {mid }}=\left(V_{L}+V_{H}\right) / 2$, and $\Delta T_{M A X}$, which defines the maximum variation of $T_{\text {exp }}$ during the search of $\bar{T}_{\text {exp }}$. The flowchart is depicted in Fig. 6.9. Flags $F_{i n c}$ and $F_{d e c}$ permit to determine when the coarse search increasing or decreasing $T_{\text {exp }}$ by $\Delta T_{\text {MAX }}$ must stop. Then, a stage of finer adjustment starts. During this stage, the variable $k$ doubles after every step of adjustment, thus speeding up greatly the process of search. But the key operation endowing the algorithm with great efficiency is the computation of $\bar{V}$ at the focal plane by realizing charge redistribution through the MOS-based RC network. This computation, taking into account the value of the time constant of the RC network (see Section 5.5.1), will be ready just after finishing the period of photointegration even in extreme cases with exposure times of only a few microseconds. Only one pixel needs then to be read out to obtain $\bar{V}$. Once $\bar{T}_{\text {exp }}$ is found, the original image is captured after deactivating the charge redistribution at the focal plane. 


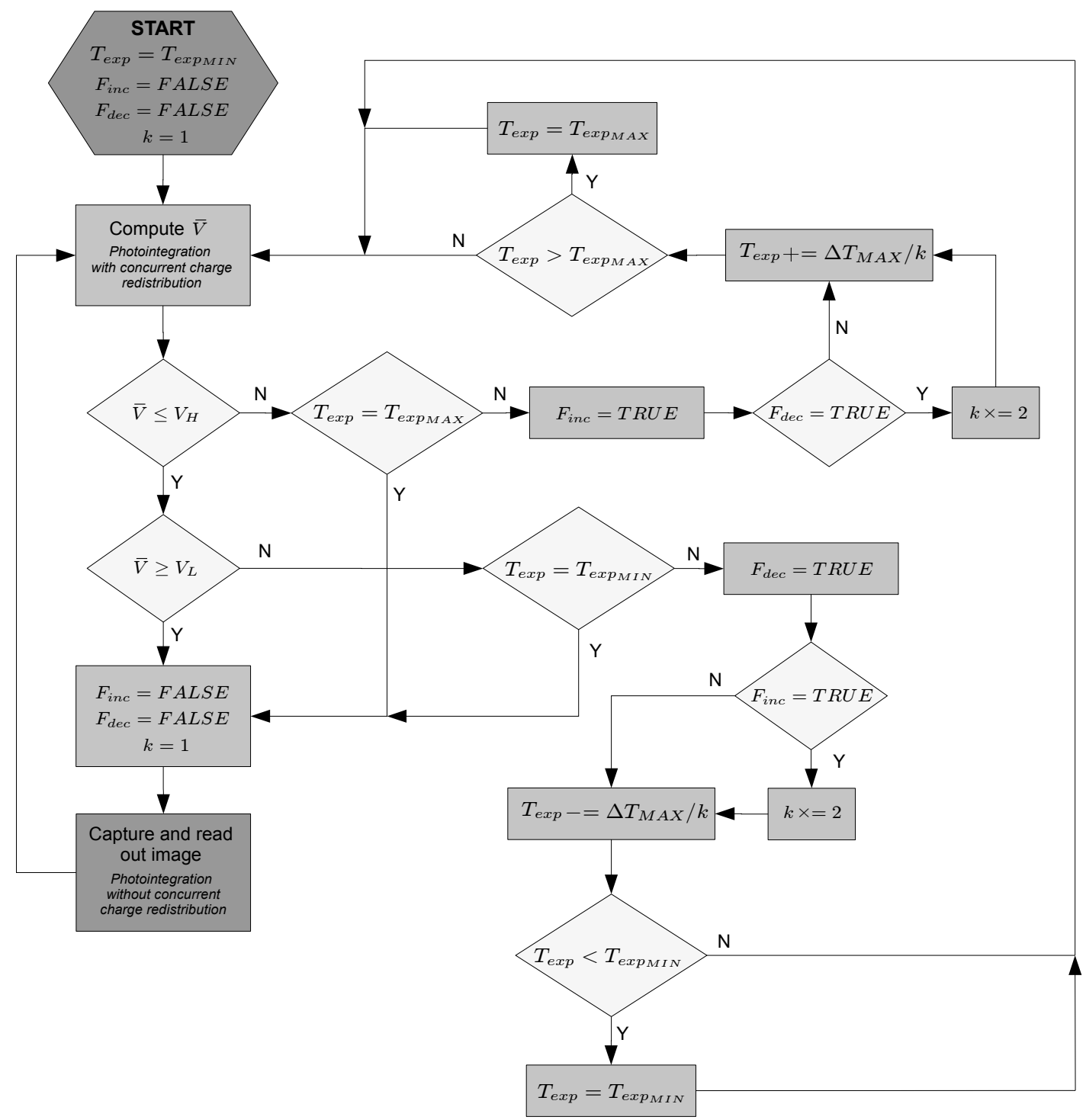

FiguRE 6.9: Flowchart of the exposure time control algorithm.

Notice that there are several tradeoffs involved in the choice of the parameters of the algorithm.

First of all, the wider the interval $\left[T_{\exp _{M I N}}, T_{\exp _{M A X}}\right]$, the better the adaptation to extreme light conditions, but the longer the search of $\bar{T}_{\text {exp }}$. On the contrary, the wider the interval $\left[V_{L}, V_{H}\right]$, the shorter this search but the worse the adaptation of the exposure time to different light conditions. Finally, the larger the value of $\Delta T_{M A X}$, the faster the search of a distant $\bar{T}_{\text {exp }}$ with respect to the current one but, on the other hand, the slower the search of a nearer $\bar{T}_{\text {exp }}$. In other words, large values of $\Delta T_{M A X}$ will work fine for constantly changing scenes whereas small values of $\Delta T_{M A X}$ will result adequate for scenes varying smoothly.

Concerning the performance of Wi-FLIP when running the algorithm, the reachable frame rate will significantly depend on the light conditions of the scene as well as the clock speed of the 


\begin{tabular}{cccc}
\hline \hline Resolution (px) & Clock frequency $(\mathbf{M H z})$ & Frame rate & Power consumption $(\mathbf{m A})$ \\
\hline \hline \multirow{3}{*}{$\begin{array}{c}\text { Full } \\
176 \times 144\end{array}$} & 13 & 0.02 & 38.4 \\
\cline { 2 - 4 } & 104 & 0.1 & 66.9 \\
\cline { 2 - 4 } & 208 & 0.18 & 105.5 \\
\cline { 2 - 4 } & 416 & 0.23 & 152.2 \\
\hline \multirow{3}{*}{$\begin{array}{c}\text { Half } \\
88 \times 72\end{array}$} & 13 & 0.08 & 38.1 \\
\cline { 2 - 4 } & 104 & 0.33 & 65.8 \\
\cline { 2 - 4 } & 208 & 0.62 & 105.6 \\
\hline \multirow{3}{*}{ Quarter } & 416 & 0.78 & 152.2 \\
\cline { 2 - 4 } $44 \times 36$ & 13 & 0.28 & 38.2 \\
& 104 & 1 & 65.6 \\
\cline { 2 - 4 } & 208 & 1.55 & 105.2 \\
\hline & 416 & & 152.1 \\
\hline
\end{tabular}

TABLE 6.3: Performance of Wi-FLIP running the exposure time control algorithm.

PXA271 processor. The clock speed will also have a decisive influence on the power consumption of the system. Table 6.3 presents some results for different resolutions and clock frequencies. The frame rate was averaged along a sequence composed of 50 frames for each case. Some full-resolution frames of the scene surveyed downloaded from Wi-FLIP are shown in Fig. 6.10. The parameters of the algorithm were set as follows: $\left[T_{\exp _{M I N}}, T_{\exp _{M A X}}\right]=[1 \mathrm{~ms}, 1000 \mathrm{~ms}]$, $\left[V_{L}, V_{H}\right]=[1.9 \mathrm{~V}, 2.1 \mathrm{~V}]$ and $\Delta T_{M A X}=128 \mathrm{~ms}$. Values of $\bar{T}_{\exp }$ ranging from around $200 \mathrm{~ms}$ to $400 \mathrm{~ms}$ were obtained. The maximum frame rate reachable therefore is around $5 \mathrm{fps}$.

As a measure of the low computational cost associated with the algorithm, we also provide some figures related to an image capture loop where exposure time adaptation is only realized at the beginning. All the images composing the sequence are therefore captured by applying the initially calculated exposure time. In such a case, the frame rates for full, half and quarter resolution with a clock speed of $13 \mathrm{MHz}$ are, respectively, $0.02,0.09$ and 0.29 . If the clock speed is set to $416 \mathrm{MHz}$, the frame rates are $0.26,0.88$ and 2.1 respectively. This means that the execution of the algorithm is not the cause of the low frame rates reached. Indeed, the bottleneck preventing $W i-F L I P$ from achieving higher frame rates is the control of the A/D conversion at FLIP-Q by Imote2. This control, that is not standard and must be therefore programmed step by step in $n e s C$, is mostly supported by GPIOs featuring very slow switching. Furthermore, the software overhead introduced by TinyOS also plays an important role. As a consequence, a great deal of clock cycles are wasted during the conversion. For instance, the frame conversion for full, half and quarter resolution at maximum clock speed, i.e. $416 \mathrm{MHz}$, takes respectively $3.9 \mathrm{~s}, 1 \mathrm{~s}$ and $0.3 \mathrm{~s}$. It is therefore mandatory for future versions of $F L I P-Q$ either the incorporation of internal digital logic realizing efficiently the ADC control or the implementation of a standard interface that speeds up this task, like for example the Quick Capture Interface provided by the PXA271 processor. 

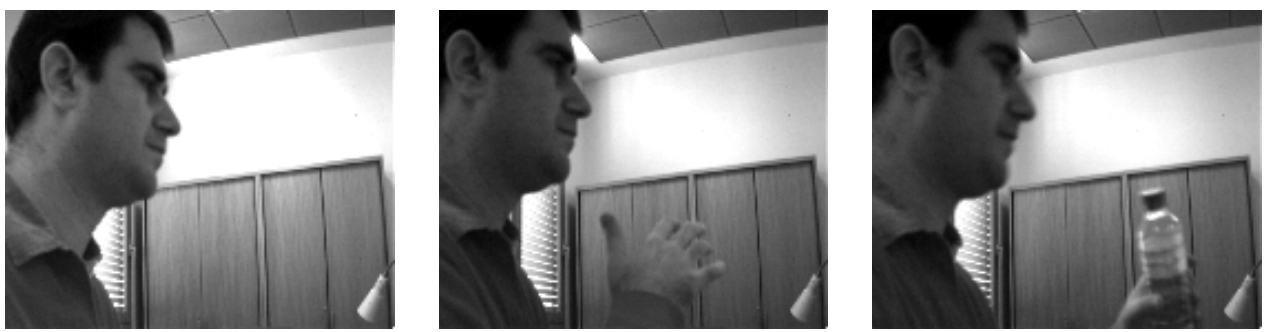

FiguRE 6.10: Scene surveyed while running the exposure time control algorithm.

\subsubsection{Edge detection algorithm}

Another operation typically needed for artificial vision applications is edge detection. We saw in Section 3.1 that this operation can be realized through Difference of Gaussians (DoG). In our case, the difference between a non-filtered image and a Gaussian-filtered version of that same image will be computed. We can afford this simplification because of the low noise associated to the frames captured by FLIP-Q, what enables the possibility of skipping the application of a first Gaussian filter to eliminate high-frequency noise. The algorithm developed firstly performs an adaptation of the exposure time as above explained. Then, a focal-plane Gaussian filter with $\sigma \geq 0.7$ is applied over the resulting image. For full resolution, this filtering is realized through time-controlled diffusion making use of the internal VCO (see Section 5.3.1). For lower resolutions, focal-plane reconfiguration and charge redistribution is applied (see Section 3.4). Finally, the absolute value of the pixel difference between the original non-filtered image and the filtered image is calculated at the PXA271 processor. The flowchart of the algorithm is represented in Fig. 6.11. Table 6.4 summarizes the results obtained for the same resolutions and clock frequencies considered for the exposure time control algorithm. The scene surveyed is also the same. Two full-resolution edge filtered images downloaded from Wi-FLIP are depicted in Fig. 6.12.

\subsection{Comparison with the state of the art}

The current version of Wi-FLIP can not still compete with the rest of vision-enabled WSN nodes reported in the literature in terms of system performance. According to the FOM defined in Section 2.3, the energy per pixel required to complete the simple algorithms just described is always over $100 \mu \mathrm{J} / \mathrm{px}$, the worst figure when compared to those of Table 2.1. The reason of this poor performance, as above commented, is the slow process of conversion and reading of the images coming from $F L I P-Q$ realized by the PXA271 processor, what leads to very low frame rates. The automatization of this process would eliminate the high latency introduced by switching GPIO ports through TinyOS. 


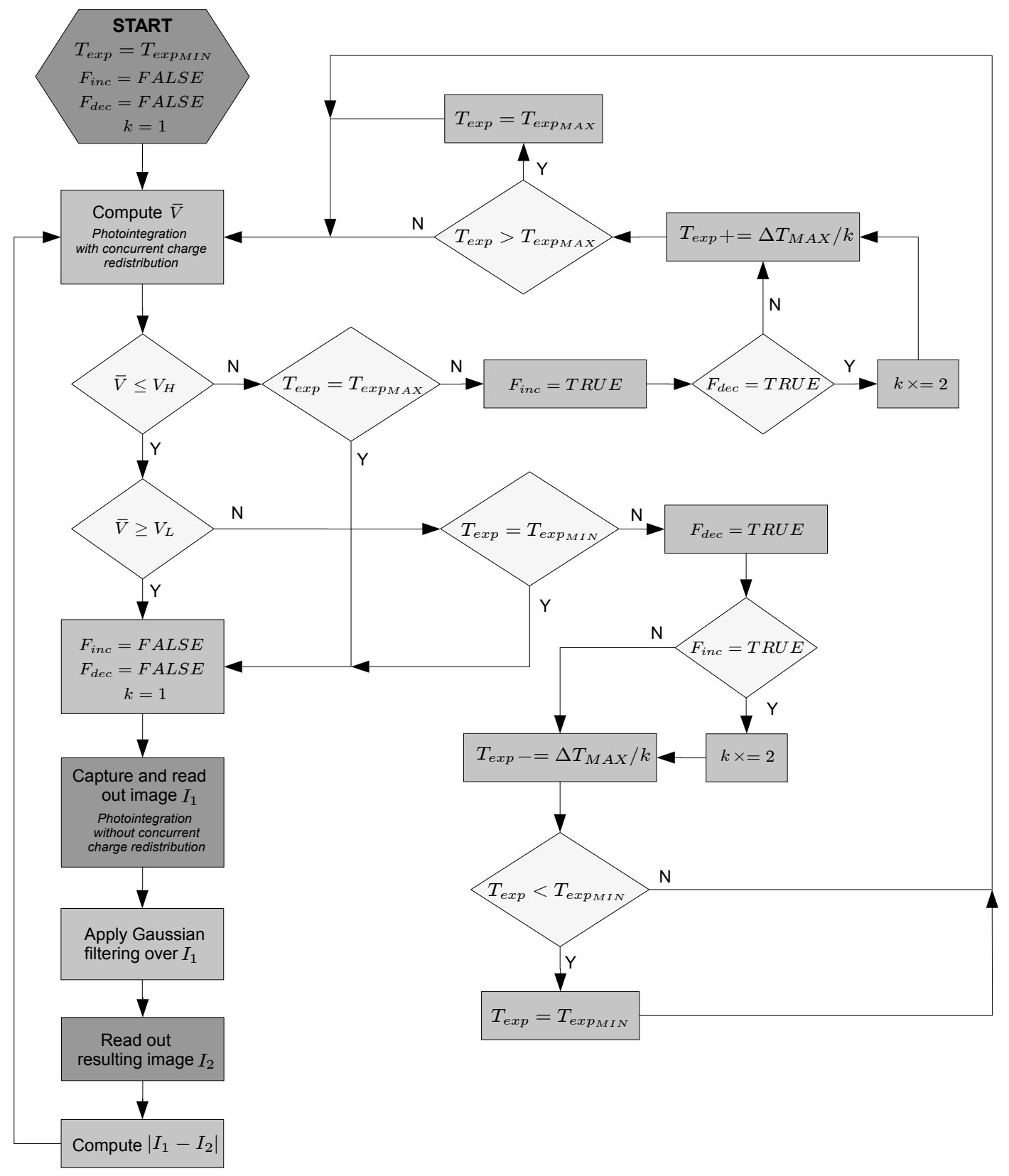

Figure 6.11: Flowchart of the edge detection algorithm. 

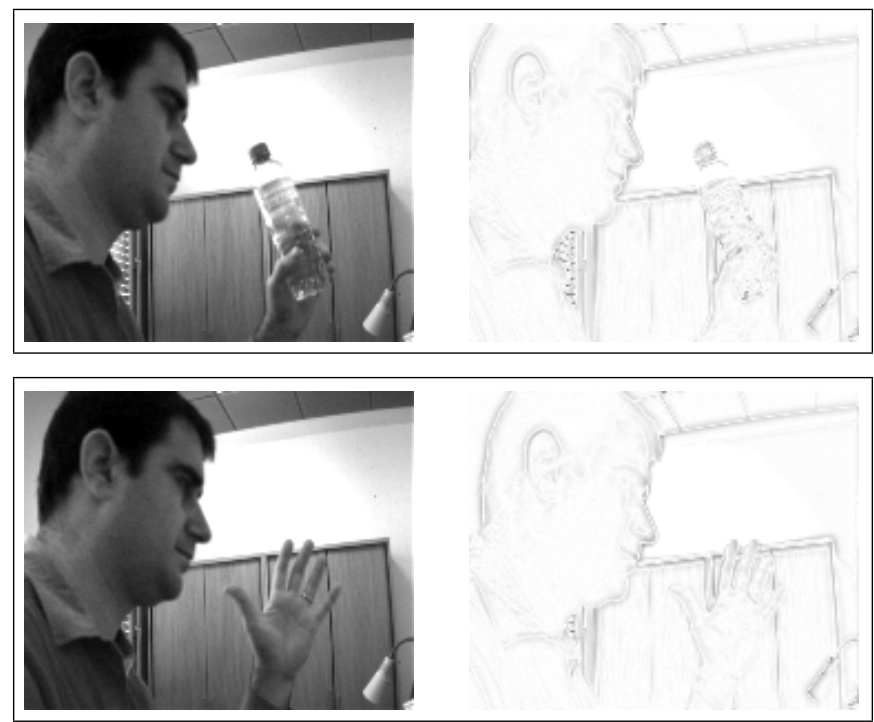

Figure 6.12: Two frames after running the edge detection algorithm.

However, if we only focus on the vision device, the prototype described in this work presents a very good performance. Table 6.5 shows the energy per pixel required exclusively for the imagers of the different platforms described in Chapter 2. For most of them, their functionality boils down to simply capturing images, applying some corrections (brightness, color etc.) and delivering them to a digital processor. Typical figures extracted from the corresponding datasheets have been used to compose the table. On the contrary, the functionality of $Q$-Eye, the smart imager of the EyeRIS ${ }^{\mathrm{TM}}$-based system, as well as of FLIP-Q is not only to capture images but also to perform massively parallel focal-plane processing in order to reduce the computational load of the subsequent digital stage. Thus, a fair comparison between these kind of smart sensors and conventional imagers is quite difficult to be established as the performance of the former ones

\begin{tabular}{cccc}
\hline \hline Resolution (px) & Clock frequency $(\mathbf{M H z})$ & Frame rate & Power consumption $(\mathbf{m A})$ \\
\hline \hline \multirow{3}{*}{$\begin{array}{c}\text { Full } \\
176 \times 144\end{array}$} & 13 & 0.01 & 38.8 \\
\cline { 2 - 4 } & 104 & 0.05 & 67.8 \\
\cline { 2 - 4 } & 208 & 0.08 & 107.8 \\
\cline { 2 - 4 } & 416 & 0.1 & 155.6 \\
\hline \multirow{3}{*}{ Half } & 13 & 0.04 & 38.7 \\
$88 \times 72$ & 104 & 0.15 & 67.1 \\
\cline { 2 - 4 } & 208 & 0.22 & 105.7 \\
\cline { 2 - 4 } & 416 & 0.25 & 153.3 \\
\hline \multirow{3}{*}{ Quarter } & 13 & 0.12 & 38.1 \\
\cline { 2 - 4 } $44 \times 36$ & 104 & 0.41 & 67.5 \\
\cline { 2 - 4 } & 208 & 0.55 & 105.9 \\
\hline & 416 & 0.59 & 153.1 \\
\hline
\end{tabular}

TABLE 6.4: Performance of Wi-FLIP running the edge detection algorithm. 


\begin{tabular}{ccc}
\hline \hline Platform & Imager & Energy per pixel (nJ/px) \\
\hline \hline Cyclops & ADCM-1700 & 27.6 \\
\hline Imote2-based & OV7649 & 4.3 \\
\hline MeshEye & ADCM-2700 & 26 \\
\hline WiCa & OM6802 & 11.9 \\
\hline FireFly Mosaic & OV6620 & 12.8 \\
\hline CITRIC & OV9655 & 4.6 \\
\hline EyeRIS & Q-Eye & no data available \\
\hline Wi-FLIP & FLIP-Q & 7.4 \\
\hline
\end{tabular}

TABLE 6.5: Comparison of the imager's performance for different vision-enabled WSN nodes reported in the literature.

will greatly depend on the specific operations being performed at the focal plane. In order to give at least an idea of this performance, we have considered for FLIP-Q the worst measured case in terms of power consumption (see Table 5.3). It corresponds to capture QCIF-resolution frames at 30fps, divide these frames into blocks of $8 \times 8 \mathrm{px}$, apply fine Gaussian filtering to these blocks by using the internal VCO at $150 \mathrm{MHz}$, compute the block energy and finally deliver the resulting $22 \times 18$-px energy-based image flow. Concerning $Q$-Eye, unfortunately no public figures are available reporting the power consumption of any of its focal-plane operators. Coming back to Table 6.5 , it can be seen that the energy per pixel needed by FLIP-Q is very close to the lowest value while delivering not raw images but elaborated representations of the scene.

\subsection{Summary}

The Wica and EyeRIS ${ }^{\mathrm{TM}}$-based platforms, described in Chapter 2, represent the first attempts of taking advantage of the inherent characteristics of early image processing in order to increase the energy efficiency of vision-enabled WSN nodes. While this objective is achieved in terms of the ratio 'power consumption'/'computational power', their power consumption as a whole makes them still too heavy for their incorporation to real WSN scenarios. Wi-FLIP enables this incorporation by integrating a prototype smart image sensor capable of carrying out very efficiently early vision tasks through analog focal-plane circuitry. This sensor features a very high performance, making the most of an ad-hoc transistor-level design that permits user-defined simplification of an image sequence. Currently, the main weakness of Wi-FLIP is its low throughput due to the slow interface between the prototype chip and the system providing further — digital — image processing and radio communication. Despite this drawback, we will demonstrate next that real applications can be still addressed. 


\section{Chapter 7}

\section{Case study: early detection of forest fires}

\subsection{Motivations and background}

Forests are an essential component of the environment. Within the global carbon cycle, they are responsible for about $30 \%$ of $\mathrm{CO}_{2}$ balance of the atmosphere [Malhi et al., 2002]. Through evapotranspiration, forests also contribute to the water cycle. Besides, they provide natural habitats to animals and plants, preserving the biodiversity of different ecosystems. In fact, it is estimated that at least $60 \%$ of all species live in tropical forests [on Biological Diversity, 2001]. Regarding their economic exploitation, forests can be considered a source of wealth. One-third of them are primarily used for production of wood and other products and 10 million people are employed in forest conservation and management around the world [FAO, 2006].

Despite their crucial importance, forests are greatly threatened, mainly by deforestation. Their total area decreased an average of 8.4 million hectares per year during the period 1990-2005 [FAO, 2006]. If this rate keeps up, all forests will disappear in less than five hundred years. Fire constitutes one of the primary agents of forest degradation. Indeed, there are regions where it is the main cause of forest destruction, e.g. the countries of the Mediterranean basin [FAO, 2007]. Forest fires do not only impact on the environment. They cause loss of human and animal lives as well as enormous economic damage. In order to minimize the effects of a forest fire, two fundamental aspects must be considered. First, early detection. Stopping the spread of a forest fire beyond 15 minutes following ignition is very difficult in most cases [INSA, 2000]. And second, precise location, which enables a fast and efficient intervention of the fire fighting resources. Therefore, apart from preventive measures, an early and precise detection is mandatory to rapidly suppress forest fires. 


\subsection{Forest fire detection: state of the art}

Traditionally, forests have been watched from lookout towers strategically situated to survey a large area. These towers house people constantly watching for fires. The surveillance task is carried out by using devices to detect smoke plumes and flames, e.g. binoculars, and then to estimate the geographical location of the fire, e.g. Osborne Fire Finders [Fleming \& Robertson, 2003]. However, this approach for forest fire detection is currently changing due to several factors. First of all, the demotivating and hard working conditions of the watchers. They have to carry out a really tedious task in a, usually, completely isolated spot. Second, rural depopulation, which is making to find personnel with an in-depth knowledge about the region to be surveyed more difficult each time. This is a crucial issue for a fast and precise location of fires as well as to dismiss false alarms. And, finally, the development of technology capable of realizing automatic detection. As a consequence, alternatives to this traditional approach have arisen. We outline them next, with emphasis on their temporal and spatial resolution, that is, on their capability to carry out early and precise detection.

\subsubsection{Ground systems}

The development of automatic ground systems has gained momentum during the last few years. Most of them take advantage of the existing lookout towers as well as other possible tall locations to install infrared and/or visual cameras surveying vast areas [Sistema Bosque, 1999; Kuhrt et al., 2001; Fire Watch, 2004; Stipanicev et al., 2010]. Infrared cameras detect the infrared radiation from fire whereas visual cameras look for smoke plumes during the day and flames during the night in the visible part of the electromagnetic spectrum. The processing for detection can be realized on-site or in a control center where the images are sent. As far as temporal and spatial resolution is concerned, these systems can detect forest fires within time intervals of minutes and, helped by Geographic Information Systems (GIS), with a high spatial resolution [Schroeder, 2004]. However, two important problems currently exist: cost and false alarms. In [Schroeder, 2005], a cost analysis of a commercial system is realized. The main conclusion is that the additional costs associated with the deployment and maintenance of the system are only compensated when the number of unmanned lookout towers is at least five, what is only suitable for very large extensions. If infrared technology is to be used, budgets become really high. Besides, numerous false alarms are delivered coming from different sources. For instance, heated objects and artificial lights when using infrared cameras [Arrue et al., 2000] or clouds moving along the horizon and dust particles when visual cameras are used [Schroeder, 2004]. It forces the existence of expert operators interacting with the system in order to dismiss false alarms. 


\subsubsection{Airborne systems}

They are based on surveillance from manned or unmanned aerial vehicles (UAVs). Early fire detection through human surveillance from planes constantly flying over the targeted area is economically unfeasible. UAVs clearly represent a better option, though still expensive. In [Merino et al., 2010], several UAVs cooperate in fire monitoring activities, allowing to cover big areas and obtaining complementary views of them. Another example is the commercial UAV presented in [Fulmar System Web Page], with capabilities to take and transmit real-time video or infrared images. Apart from high cost, there are other drawbacks for this kind of systems to carry out early detection. For example, legal restrictions for UAVs autonomously hovering across vast areas. Also the environmental impact of a fleet of UAVs constantly flying over a region must be taken into account.

\subsubsection{Satellite-based systems}

In these systems, the fire detection is realized by analyzing the multi-spectral imagery coming from satellites. The most widely used satellites are the NOAA Polar Orbiting Environmental Satellites whose payload contains an Advanced Very High Resolution Radiometer (AVHRR) [AVHRR Web Page]. This sensor acquires information in the green, red, mid-infrared and thermal part of the spectrum. Other satellites used for fire detection are [San-Miguel-Ayanz et al., 2005] the NASA EOS satellites, with the Moderate Resolution Imaging Spectroradiometer (MODIS) sensor [MODIS Web Page] on board providing better spatial resolution than the AVHRR. A comparison between AVHRR and MODIS is realized in [Cahoon et al., 2000]. The smallest area of flame fronts that can be detected are $435 \mathrm{~m}^{2}$ and $213 \mathrm{~m}^{2}$ respectively. Regarding the temporal resolution achieved for these systems, it depends on the re-visit time of the satellites whose order of magnitude is usually several hours.

The FUEGO mission [INSA, 2000] was conceived with the intention of overcoming the problems of resolution and achieving monitoring and early fire detection from a satellite system. The system was composed of a 12-satellite LEO constellation for distributed acquisition, a network of ground stations for local data processing and progressive detection algorithms for short detection time. The re-visit time was reduced to $30 \mathrm{~min}$. However, this system was not finally implemented. Instead, a deep review of the initial requirements was realized during the FUEGOSAT mission [Battistelli et al., 2005]. In order to reduce technological complexity and costs, the goal of early fire detection was removed. The FUEGOSAT mission defined a constellation of only 3 satellites operating on 3 different orbital planes. This constellation only achieves forest fire monitoring, not early detection. 


\subsubsection{Other approaches}

New approaches for forest fire detection are being currently studied by the research community. Amongst them, the most outstanding are those based on WSN and LIDAR technology. WSNbased systems [Yu et al., 2005; Doolin \& Sitar, 2005; Chaczko \& Ahmad, 2005; Son et al., 2006; Hefeeda, 2007; Machado et al., 2010] analyze regular observations of environmental conditions, such as temperature, relative humidity or wind speed, taken at different locations of the region surveyed. By means of these observations, they are capable of determining the fire ignition probability and its intensity if it materializes. Therefore, these systems are designed to predict rather than detect fires. This feature constitutes in turn its main drawback since, for example, arsons can not be predicted. Obviously, the sensors deployed could still detect the increase of temperature and decrease of barometric pressure and humidity from flame fronts before being burnt. Even the heat coming from a nearby fire can be used by a sensor as an energy source in order to send a warning signal [Siden et al., 2007]. But it demands a really dense deployment of sensors enabling the detection of fires before wide spread. We will see shortly how to get around this problem by incorporating vision capabilities into the sensors.

Also LIDAR-based techniques can be applied to detect forest fires [Utkin et al., 2002, 2010]. In this case, a laser produces short and intense radiation pulses which propagate through the atmosphere. When the laser beam hits a target, a part of the radiation is backscattered and collected by a receiver, where it is converted into an electric signal. This signal is amplified, digitized, and stored in a data-acquisition unit as a function of the time passed since the laserpulse emission. LIDARs for automated surveillance are supplemented with a signal analysis system, capable of discriminating signatures corresponding to forest fires and issuing an alarm signal containing information about the target that caused the alarm and its location. One of the main advantages of this technology is that smoke plumes can be detected during both day and night. On the contrary, high cost and potential danger to the eye caused by the probing laser beam, which in turn implies reduced range detection, constitute its drawbacks for the time being.

\subsection{Vision-enabled WSN for early detection of forest fires}

Ground systems are currently the only ones capable of detecting a fire in few minutes since it started. They rely on cameras that watch large areas. This means that a reduced number of them suffices to survey an extensive region. At the same time, it also means that detection is quite difficult as smoke can appear at really distant locations. On the other hand, WSNbased systems trust in low-cost sensors which only monitor their surrounding environmental conditions. Thus, a really dense sensor deployment would be mandatory to accomplish early 
detection. We propose to merge these two approaches in order to achieve robust, scalable and reliable detection of forest fires with appropriate temporal and spatial resolution to rapidly detect them. The resulting system would be based on a sparse deployment of smart visionenabled sensors composing a wireless network. These sensors, in addition to periodically sample their environmental conditions, would run on-site a tailored vision algorithm to detect smoke arising from small vegetation areas. We mean small compared to the areas surveyed by cameras in ground systems. When a sensor detects smoke, a warning message is sent to a control center by multihopping through the network. A sketch of the system is depicted in Fig. 7.1, where $A_{S_{i}}$ represents the area surveyed by the sensor $S_{i}$. For the sake of clarity, only the area watched by the sensor detecting smoke is depicted.

The proposed system presents several advantages when compared to automatic ground systems, namely:

- Robustness: The failure of a sensor only affects to a very small fraction of the surveyed region. For a system based on cameras, the failure of one of them implies that a large area stops being watched.

- Scalability: On watching small areas, the layout of the sensor deployment can be adapted to the extension, orography and fire danger index of the region to be surveyed.

- Reliability: The vision sensors do not analyze landscape images but small vegetation areas. We are thus making detection easier and avoiding typical sources of false alarms like clouds or smoke arising from factories.

- Better temporal resolution: Thanks also to the small size of the areas surveyed by the sensors, smoke can be detected within time intervals of seconds instead of minutes.

- Simpler smoke location: Complex GIS software is necessary for smoke location in commercial ground systems. In a system based on smart vision sensors, each sensor could store the geographic reference of the area it is surveying and include it in the alarm message. It would suffice to rapidly locate the fire.

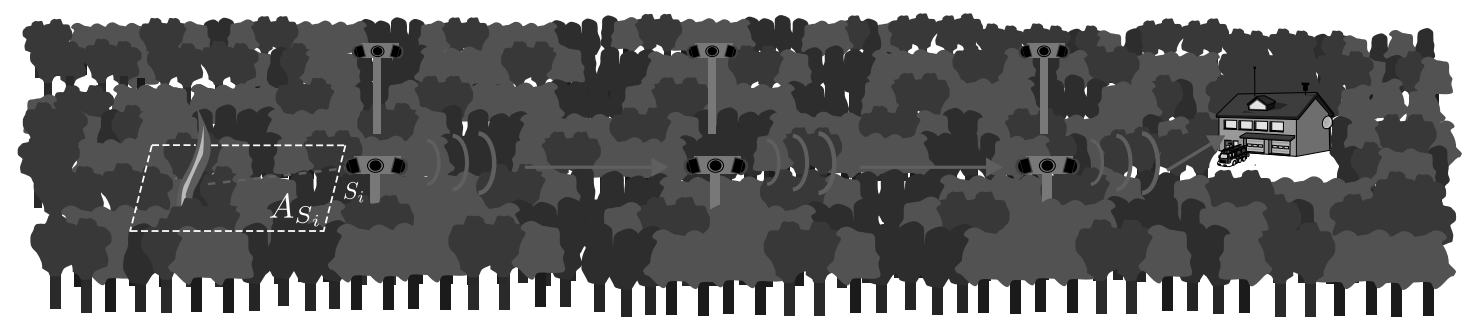

FiguRE 7.1: Sketch of the proposed system 
With respect to the WSN-based systems reported so far in the literature, the incorporation of vision makes dense deployments for early detection unnecessary. Now the sensors do not have to be very close to the fire to detect its presence. This implies a significant reduction in the number of sensor nodes, what constitutes itself a significant step further in terms of cost and maintenance of the system as a whole.

Of course, the goodness of the proposed system is not accomplished for free. It is fundamentally based on the achievement of the primary motivation of this work, i. e. the efficient integration of vision capabilities into the sensing nodes. Keep in mind that we want sensors to constantly run a vision algorithm, ideally during both day and night, at the minimum possible energy cost in order to prolong the lifetime of their batteries as much as possible. So far, our work has been focused on the implementation of low-power vision hardware from a general point of view. It is time now to make use of this hardware for a very particular case.

\subsection{A vision algorithm for smoke detection}

Numerous vision algorithms have been reported in the literature intended to detect smoke [de Vries \& Kemp, 1994; den Breejen et al., 1998; Vicente \& Guillemant, 2002; Gómez-Rodríguez et al., 2002; Toreyin et al., 2007; Krstinic et al., 2009]. All of them analyze images coming from remote cameras surveying large areas. Thus, they have to deal with cloud motion, dust etc. in order to reduce the false alarm rate. On the contrary, in the system above proposed, the sensors watch small areas and, consequently, most of the pixels of the images processed will correspond to nearby vegetation. This means that the potential sources of false alarms are totally different. We must now filter the motion of tree leaves, birds or even people walking around.

As a first step, we are going to divide the image plane into regular regions with a size of $W \times H$ pixels. The processing is then focused only on the mean value of the pixels composing each region. This reduced scene representation can be very efficiently achieved by making use of the multiresolution primitive (see Section 3.3) implemented by the FLIP-Q prototype. Despite its simplicity, we will demonstrate that such representation suffices to carry out a reliable smoke detection.

In terms of image processing, the main effect caused by smoke arising against a vegetation background is the increase of the luminance in the regions affected. Indeed, if we are processing RGB images, the effect would not only be the increase of each component but also their equalization [Chen et al., 2006]. And even going further, we have found that the most sensitive component to the presence of smoke in such conditions is the blue (B) component. Consider Fig. 7.2. We have marked a zone within a scene whose background is mainly constituted by vegetation. We represent then the intensity histogram of the RGB components and the luminance under two 

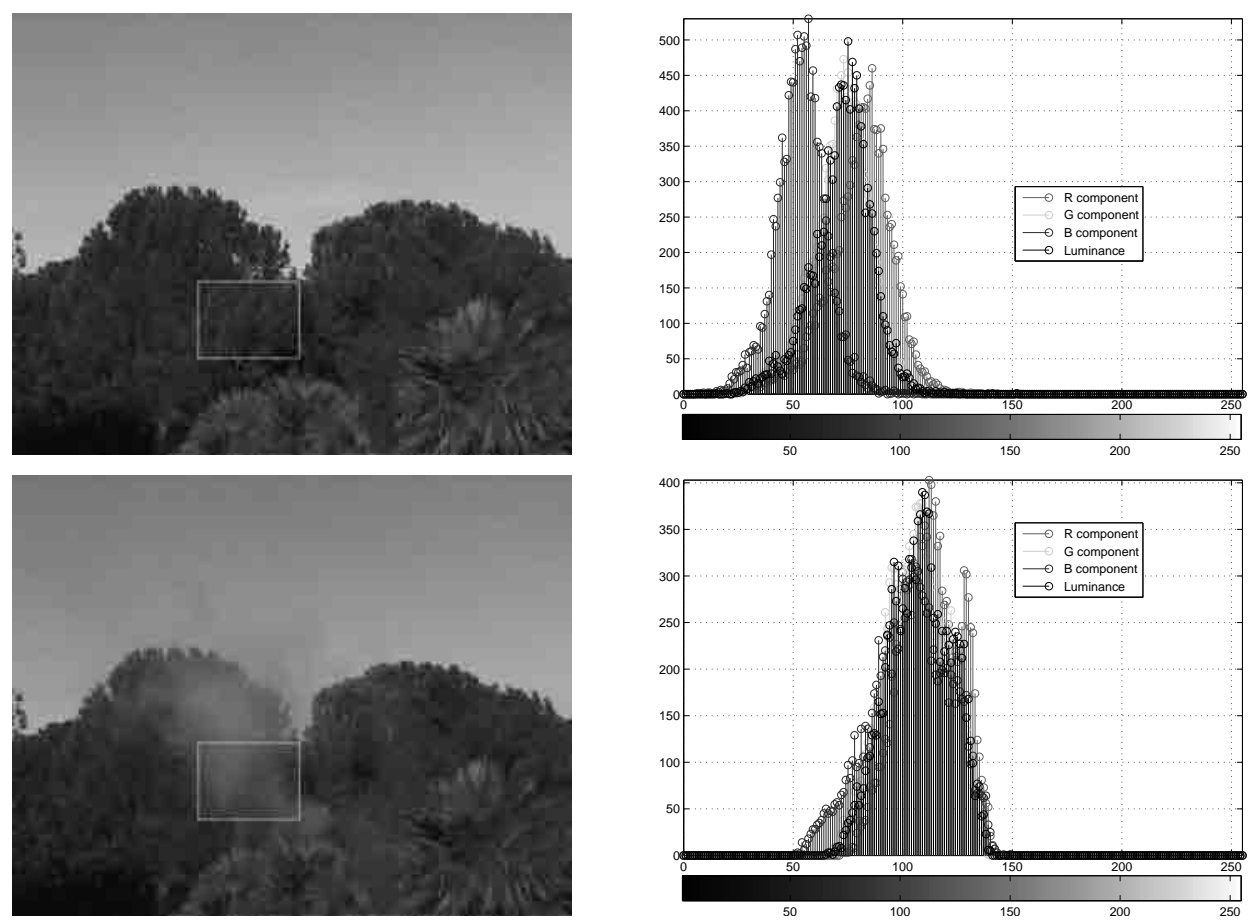

FiguRE 7.2: Intensity histogram of the RGB components and luminance of a vegetation zone without smoke and in presence of smoke.

situations: without smoke and in presence of smoke. It can be seen that, without smoke, the B component presents the lowest values of intensity. When smoke appears, the RGB components and the luminance increase and equalize their intensities. Another example is shown in Fig. 7.3. In order to establish a numerical reference, we have calculated the normalized average increase, referred to the background, suffered by each component of the pixels within the marked zones when smoke appears. The results are summarized in Table 7.1. We can see that, in both examples, the appearance of smoke among vegetation means a greater increase for the $\mathrm{B}$ component than for the $\mathrm{R}$ and $\mathrm{G}$ components and the luminance. Therefore, the $\mathrm{B}$ component is the most sensitive to the presence of smoke. In our case, we will sense the presence of smoke from the analysis of the light intensity sensed by FLIP-Q, which, unfortunately, is not only constrained to the B component at all. Indeed, its responsivity covers wavelengths from $400 \mathrm{~nm}$ to $1000 \mathrm{~nm}$ approximately.

The first condition of the algorithm has to do with a possible increase detected at any of the regions comprising the image plane division. A region $(k, l)$ will be considered a candidate to contain smoke when its foreground intensity $I_{k l_{F}}$ fulfills the following equation:

$$
I_{k l_{F}} \geq I_{k l_{B}}+q
$$

where $I_{k l_{B}}$ is the background intensity of that same region $(k, l)$ and $q$ is a parameter indicating 

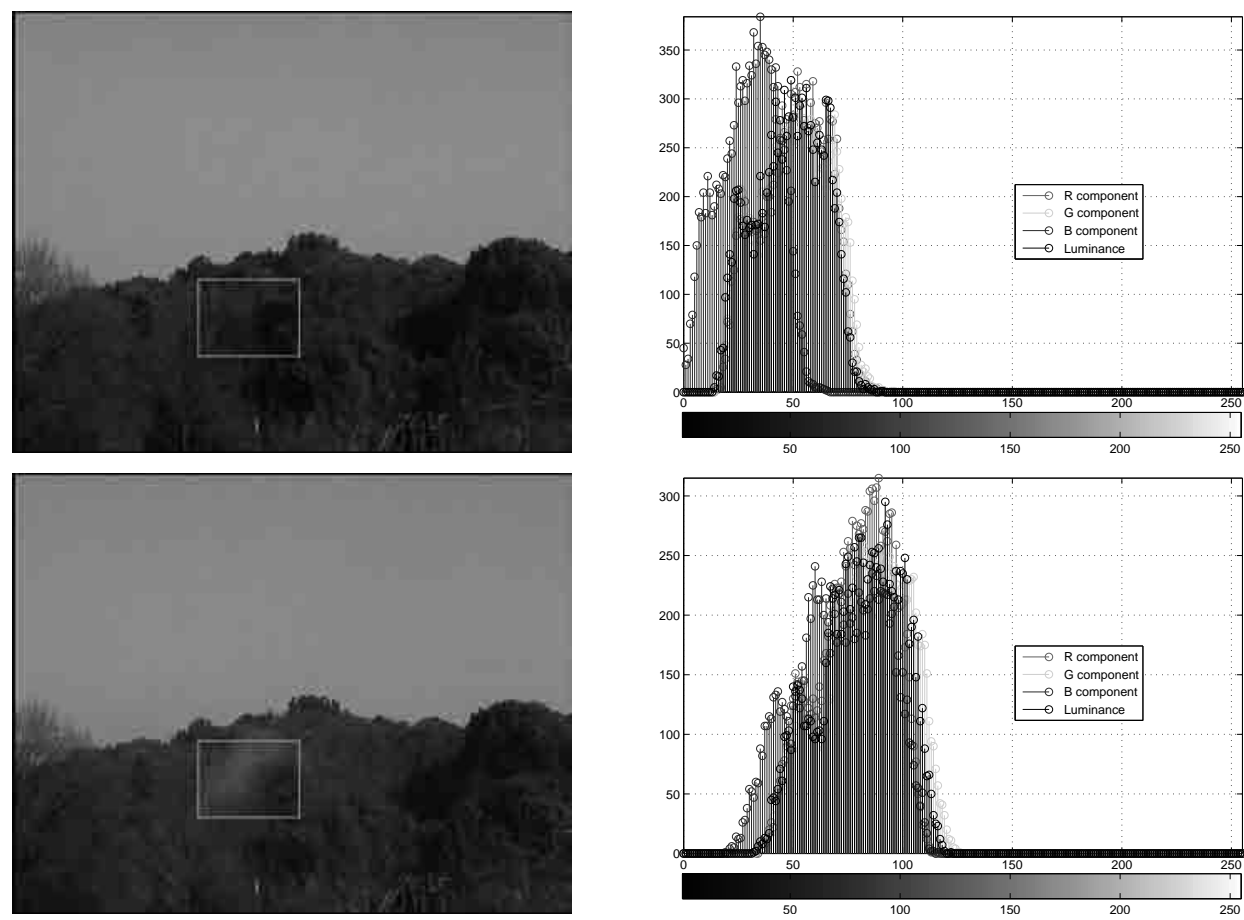

FiguRE 7.3: Intensity histogram of the RGB components and luminance of a vegetation zone without smoke and in presence of smoke.

\begin{tabular}{lcccc}
\hline & R component & G component & B component & Luminance \\
\hline Fig. $\mathbf{7 . 2}$ & $12.5 \%$ & $13.4 \%$ & $19.5 \%$ & $13.8 \%$ \\
\hline Fig. $\mathbf{7 . 3}$ & $11 \%$ & $12.8 \%$ & $16.4 \%$ & $12.7 \%$ \\
\hline
\end{tabular}

TABLE 7.1: Normalized average increase, referred to the background, suffered by each component of the pixels within the zones marked in Fig. 7.2 and Fig. 7.3, when smoke appears.

the minimum increase of intensity which must suffer a region to consider it candidate to contain smoke. Note that Eq. 7.1 demands the existence of a background model. Since the background of the scenes inspected by the algorithm, mostly composed of vegetation, will rarely undergo significant changes, it will be simply represented by frames updated every $T_{B}$ seconds, provided that no candidate region has been detected from the previous frame. If candidate regions are being analyzed, we will wait until confirming or dismissing the presence of smoke. If dismissed, the background is finally updated with the next frame captured. The parameter $T_{B}$ can be therefore expressed as:

$$
T_{B}=n T_{F} \quad\{n \in \mathbb{N}\}
$$

where $T_{F}$ is the time interval determining the rate at which foreground images are captured and processed to detect smoke and $n$ is the number of foreground images which must be analyzed as minimum until the next background update. 
Once candidate regions have been detected, the algorithm looks for spatio-temporal patterns which are characteristic of the smoke dynamics. This stage of the algorithm is divided into two phases: a detection phase and a confirmation phase. The detection phase starts when the first candidate regions are discovered, an instant denoted as $t_{0}$, and finishes at $t=t_{D}$. Then the confirmation phase is started. It will last $T_{C}$ seconds at most if smoke is really present, finishing at time $t=t_{C}$ by sending an alarm message. The internal processing at both phases is described next.

First of all, in order to consider that smoke is present at the scene, a minimum number of candidate regions must exist. Let us define $N(t)$ as the number of candidate regions at time instant $t$. This parameter can change every $T_{F}$ seconds, that is, with every foreground image captured. During the confirmation phase, the following expression must be fulfilled:

$$
N(t) \geq N_{M I N} \quad\left\{t \in\left[t_{D}, t_{C}\right]\right\}
$$

where $N_{M I N}$ represents the minimum number of candidate regions to consider smoke. Below that, changes are associated to a different source and the confirmation phase is interrupted, returning to the pre-detection state.

Another important characteristic of the smoke dynamics is its gradual appearance into the scene. Once the first candidate regions are detected, new ones must gradually appear until reaching at least $N_{M I N}$ at $t=t_{D}$. This fact can be described by means of two conditions. The first one is:

$$
t_{D}-t_{0} \leq T_{D_{M A X}}
$$

where $T_{D_{M A X}}$ represents the maximum time interval within which smoke must appear once the first candidate regions are detected. The second condition is:

$$
N(t)-N\left(t-T_{F}\right) \leq \Delta_{M A X} \quad\left\{t \in\left[t_{0}, t_{C}\right]\right\}
$$

where $\Delta_{M A X}$ expresses the maximum permitted growth of candidate regions between two consecutive foreground images during the smoke dynamics. The effect of not fulfilling Eq. (7.4) is again the cancellation of the detection and the return to the pre-detection state. This is because the system considers that the source of the changes is too slow to behave as smoke, e. $\mathrm{g}$. fog. Failing to hold Eq. (7.5) means that the considered object grows faster than smoke, and it therefore comes from a different source, e. g. a bird passing by.

Finally, smoke does not appear as candidate regions scattered throughout the scene. On the contrary, it is formed by compact clusters. Let us define $Z(t)$ as the number of 8 -connected 
candidate regions. Just like $N(t), Z(t)$ can change with every foreground image. A proper compactness condition for smoke can be described as:

$$
Z(t) \leq Z_{M A X} \quad\left\{t \in\left[t_{0}, t_{C}\right]\right\}
$$

being $Z_{M A X}$ the maximum permitted number of 8-connected candidate regions during the smoke dynamics. In other words, failing to hold Eq. (7.6) means that a different and spread source is generating the changes, e.g. a flock of birds.

The spatio-temporal dynamics of smoke can be therefore summarized as follows: a minimum number of candidate regions $N_{M I N}$ must appear once the first ones are discovered at $t=t_{0}$. The time instant at which $N_{M I N}$ is reached, $t=t_{D}$, establishes the end of the detection phase and the beginning of the confirmation phase. Besides, $t_{D}$ must fulfill Eq. (7.4), accounting in this way for the time scale of the gradual appearance of smoke into the scene. During the confirmation phase, whose duration is determined by $T_{C}$, the number of candidate regions must satisfy Eq. (7.3). Thus, we take into account that smoke does not disappear suddenly from the scene. Finally, Eq. (7.5) and Eq. (7.6) must be fulfilled during both the detection phase and the confirmation phase. The first one codifies the maximum growth rate of smoke during its propagation across the scene. The second accounts for its compactness.

The flowchart of the algorithm is depicted in Fig. 7.4. There are two aspects of this flowchart that are worth to be briefly commented. First of all, notice that the value of $N(t)$ is replaced by 0 whenever dynamics other than smoke is detected. This is necessary to dismiss false candidate regions when the condition defined by Eq. (7.5) is checked for the next image of the sequence. Secondly, let us analyze the condition $t-t_{0} \leq T_{D_{M A X}}$. If it is fulfilled, the possible value of $t_{D}$ at that time instant, $t_{D}=t$, satisfies Eq. (7.4). Therefore, the algorithm must continue checking conditions over that frame as all the conditions established to detect smoke could be eventually fulfilled. On the contrary, if the condition $t-t_{0} \leq T_{D_{M A X}}$ is not satisfied, the possible assignment $t_{D}=t$ at that time instant does not meet Eq. (7.4) and the current value of $t_{D}$ must be checked. If it equals -1, Eq. (7.4) will never be fulfilled by the dynamics detected as no value was previously set for $t_{D}$. However, a value other than -1 does imply that a value for $t_{D}$ was previously assigned that satisfied Eq. (7.4). In such a case, the algorithm must continue checking conditions over that frame as, again, all the conditions established to detect smoke could be eventually met.

\subsection{Algorithm setting and preliminary tests}

In order to set the numerical values of the different parameters of the algorithm and test the algorithm itself, we recorded some sequences at a natural environment, specifically at a public 


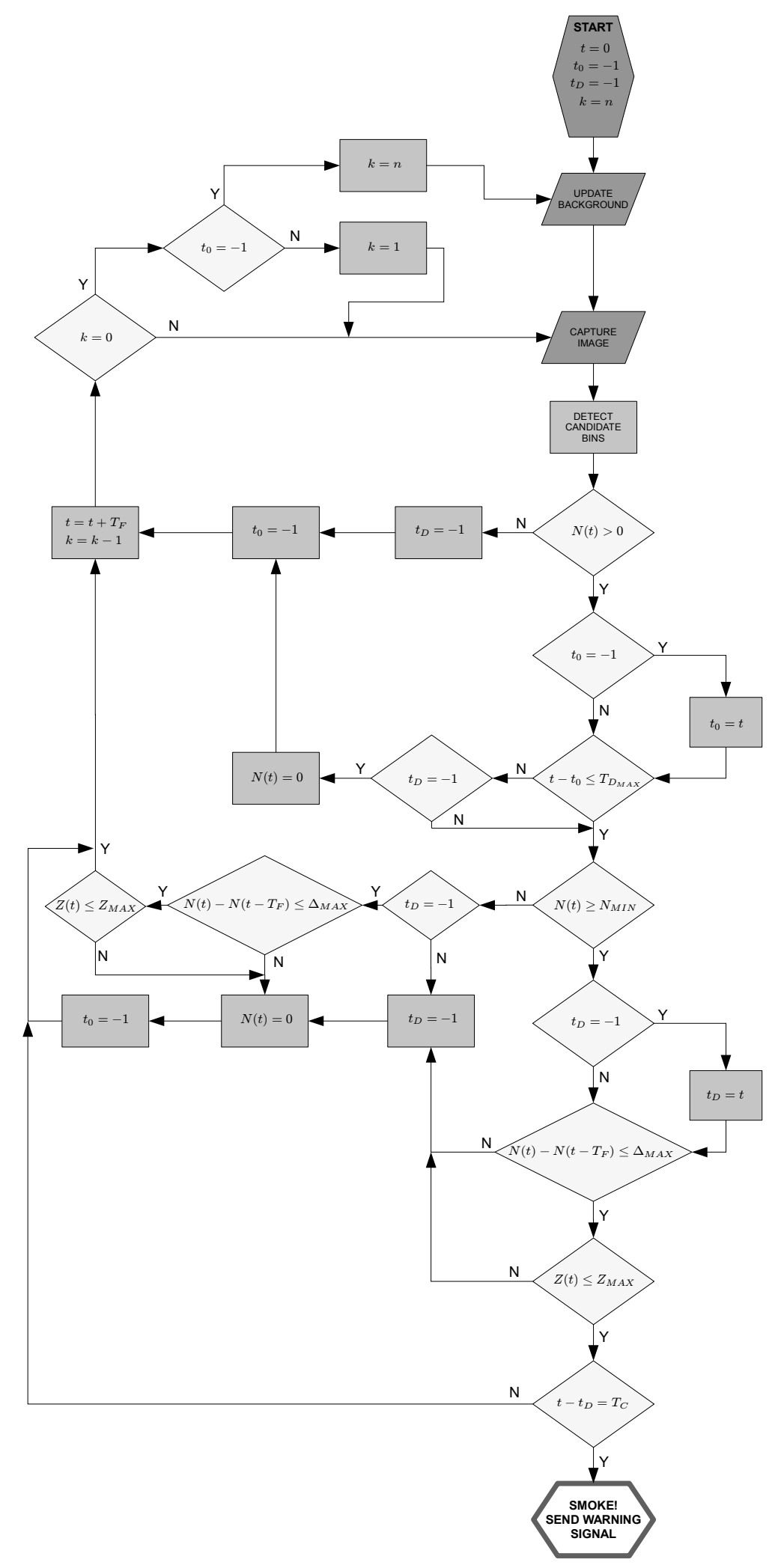

FiguRE 7.4: Flowchart of the smoke detection algorithm. 
park called Parque del Alamillo [Parque del Alamillo Web Page], in Seville. Approximately 80 minutes were recorded containing 16 sequences of gradual appearance of smoke following its natural evolution in scenes whose background was basically vegetation. Three different camcorders and commercial pyrotechnic smoke generators were used. In order to extract the parameters, 9 out of the 16 sequences were analyzed. The rest was used to test the algorithm. Also sequences without smoke were recorded to check the false alarm rate. All this material, in PAL format $(720 \times 576 \mathrm{px}, 25 \mathrm{fps})$, can be found in a web portal [V-MOTE Web Page] designed ad-hoc to house information related to the project within which this work has been developed.

We must say that, unfortunately, the maximum vegetation area covered by the cameras during the recordings was around $1000 \mathrm{~m}^{2}$. Due to the orographic features of the scenario and the available means, it was not possible to record larger areas. The algorithm has been therefore adjusted and tested for regions of this extension or below - the minimum area recorded was around $200 \mathrm{~m}^{2}$. Anyway, the accumulative nature of the presence of smoke in a scene makes us optimistic about the good behavior of the algorithm in bigger extensions, obviously with a limit. For too large areas, smoke will appear too slow for the spatio-temporal dynamics coded in the algorithm and will be therefore dismissed.

\subsubsection{Parameter setting}

The most important parameter of the algorithm is $T_{F}$. On one hand, it must be correlated with the temporal scale of the smoke dynamics. On the other hand, the larger its value, the lighter the processing load associated with the algorithm and consequently the lower the power consumption. To set $T_{F}$, we have implemented a very simple motion detector where the first frame of every sequence analyzed is considered the background. Foreground motion is then determined at pixel level for subsequent frames. Those pixels changing more than a certain threshold will belong to the foreground. By empirically adjusting this threshold in such a way that only pixels representing smoke are segmented, we can obtain approximately the number of smoke pixels per frame, depicted in Fig. 7.5 for the 9 sequences analyzed. This magnitude, highly dependent of the temporal dynamics of smoke, presents a very smooth variation. Indeed, most of the abrupt changes can be tracked by sampling the sequences every second. Thus, we conclude to set $T_{F}=1 \mathrm{~s}$. The remaining parameters are adjusted taking into account this value of $T_{F}$, that is, the sequences will be sampled every second from now on.

Regarding the size of the elementary block of the image plane division, $W \times H$, we have computed the average variation of the number of pixels affected by smoke during its appearance in the scene, represented in Fig. 7.6. This variation constitutes an estimation of the average size of the regions successively affected by smoke when it is spreading across the scene. It can be seen that the minimum value is approximately 200 pixels per second - bear in mind that the sequences are 


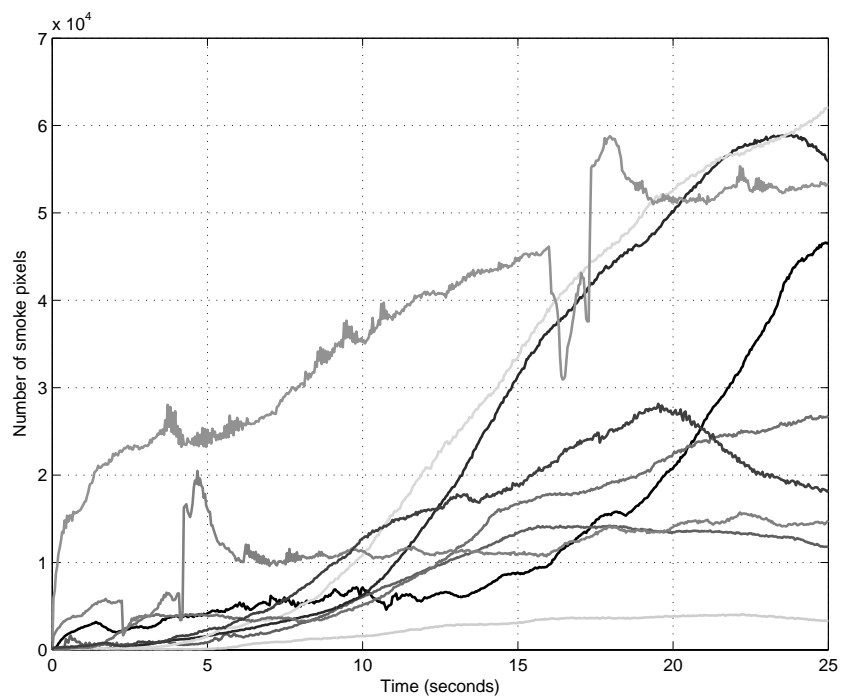

FiguRE 7.5: Number of smoke pixels per frame for each of the 9 different sequences analyzed.

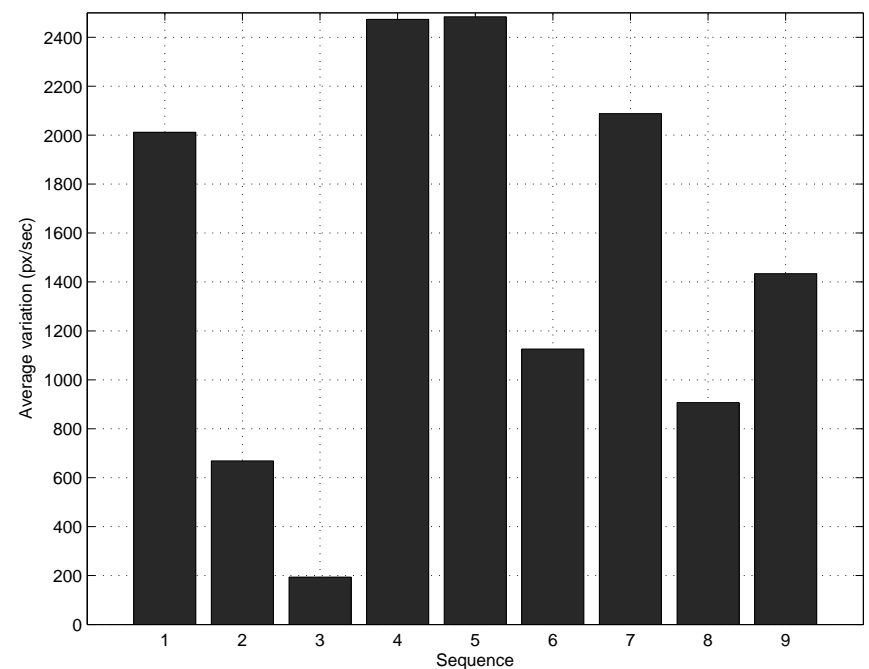

FIGURE 7.6: Average variation of the number of pixels affected by smoke for each sequence.

already sampled. We therefore decide to set $W \times H=15 \times 12 \mathrm{px}$. The dimensions of this elementary block keep the ratio 'width'/'height' of the full-resolution frame.

The next parameter considered is $q$. In this case, we have simply computed the average increase, normalized to the signal range, undergone by the values of the pixels affected by smoke. The result is depicted in Fig. 7.7. Accordingly, we set $q=10 \%$, where the percentage is referred to the signal range.

Applying Eq. (7.1) with the values of $W, H$ and $q$ just set, we can obtain the number of candidate regions along every sequence, as depicted in Fig. 7.8. It can be seen that the minimum value reached is 17 . We set $N_{M I N}=14$ in order to concede a margin of three candidate regions. This choice implicitly sets $T_{D_{M A X}}=20 s$ and $T_{C}=4 s$. At this point, it is easy to adjust the value 


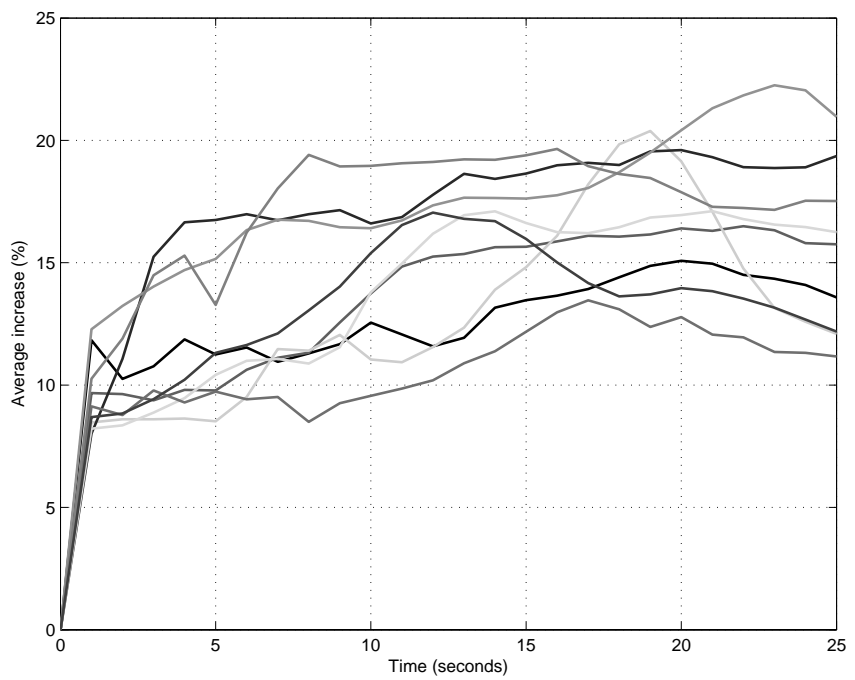

FiguRE 7.7: Normalized average increase of the pixels affected by smoke.

of $\Delta_{M A X}$ too. We have computed, from the data defining Fig. 7.8, the maximum growth rate of candidate regions for each sequence. The greatest value found is 30 regions per second. We therefore set $\Delta_{M A X}=30$.

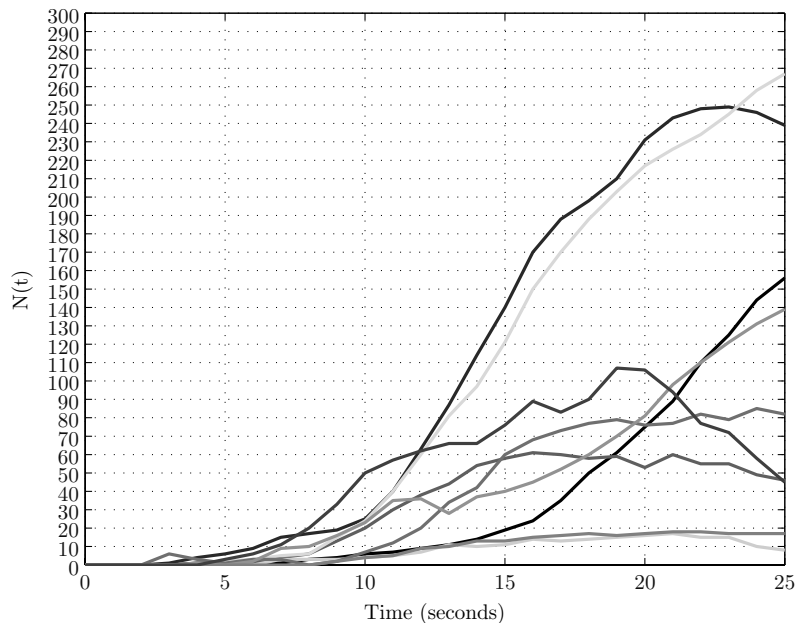

FigURE 7.8: Representation of $N(t)$ for each of the 9 different sequences analyzed.

There are two parameters left: $Z_{M A X}$ and $T_{B}$. To determine $Z_{M A X}$, consider the Fig. 7.9, where the number of 8-connected candidate regions along every sequence is depicted. We can see that the maximum value is 6 . Besides, it is reached once only in one of the sequences. Therefore, we set $Z_{M A X}=6$, without additional margin. Regarding $T_{B}$, longer recordings are mandatory in order to extract an accurate value of this parameter. Even for the longest sequence analyzed, whose duration is $458 \mathrm{~s}$, smoke detection was possible without updating the background reference. As an initial guess, we set $T_{B}=300 \mathrm{~s}$, that is, $n=300$. 


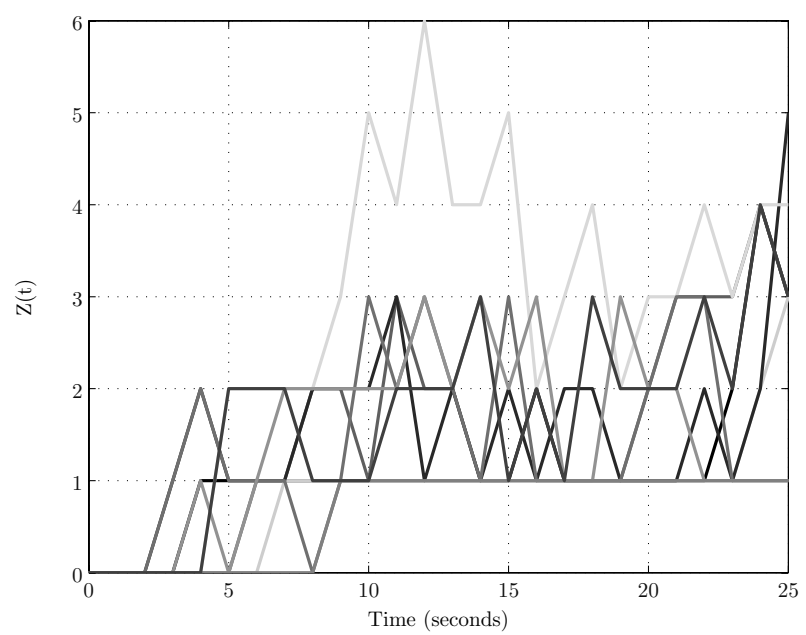

Figure 7.9: Representation of $Z(t)$ for the sequences analyzed.

\subsubsection{Results of the tests}

Once the parameters were adjusted (see Table 7.2), we applied the algorithm to the 7 smoke sequences that were not used for the setting process. Smoke was detected in all of them. The algorithm was also applied to the sequences without smoke. No false alarm was triggered despite different sources of motion like birds or tree leaves. Therefore, as far as this reduced test bench is concerned, the algorithm achieves a reliability of 100\%. As an example, we represent in Fig. 7.10 the dynamics of each and every block composing the image plane division for three sequences until the time instant at which smoke is detected. Each region is normalized with respect to its value in the background representation. Note that the time instant at which smoke begins to appear in the scene can be easily distinguished due to the progressive increase suffered by the regions affected.

\subsection{On-site smoke detection}

With the vision algorithm tuned for reliable detection, we considered the possibility of its implementation in a commercial autonomous system before programming it into Wi-FLIP. Thus, we could carry out on-site surveillance while checking the algorithm in other scenarios and facing potential problems of autonomous operation. The system chosen was the EyeRIS ${ }^{\mathrm{TM}} \mathrm{v} 1.2$ platform, sketched in Section 2.2.7. The reasons behind this choice were the availability of this system in the laboratory when we finished the preliminary tests of the algorithm and the application development kit which it is provided with, permitting a fast implementation of standalone applications.

The only modification of the algorithm for its implementation in EyeRIS ${ }^{\mathrm{TM}}$ was the image plane division. The elementary block was adjusted to the QCIF resolution $(176 \times 144 \mathrm{px})$ of $Q$-Eye, 


\begin{tabular}{cc}
\hline \hline Parameter & Value \\
\hline \hline$T_{F}$ & $1 s$ \\
\hline$T_{B}$ & $300 s$ \\
\hline$W \times H$ & $15 \times 12 \mathrm{px}$ \\
\hline$q$ & $10 \%$ \\
\hline$N_{M I N}$ & 14 \\
\hline$T_{D_{M A X}}$ & $20 s$ \\
\hline$T_{C}$ & $4 s$ \\
\hline$\Delta_{M A X}$ & 30 \\
\hline$Z_{M A X}$ & 6 \\
\hline \hline
\end{tabular}

TABLE 7.2: Summary of the algorithm setting.

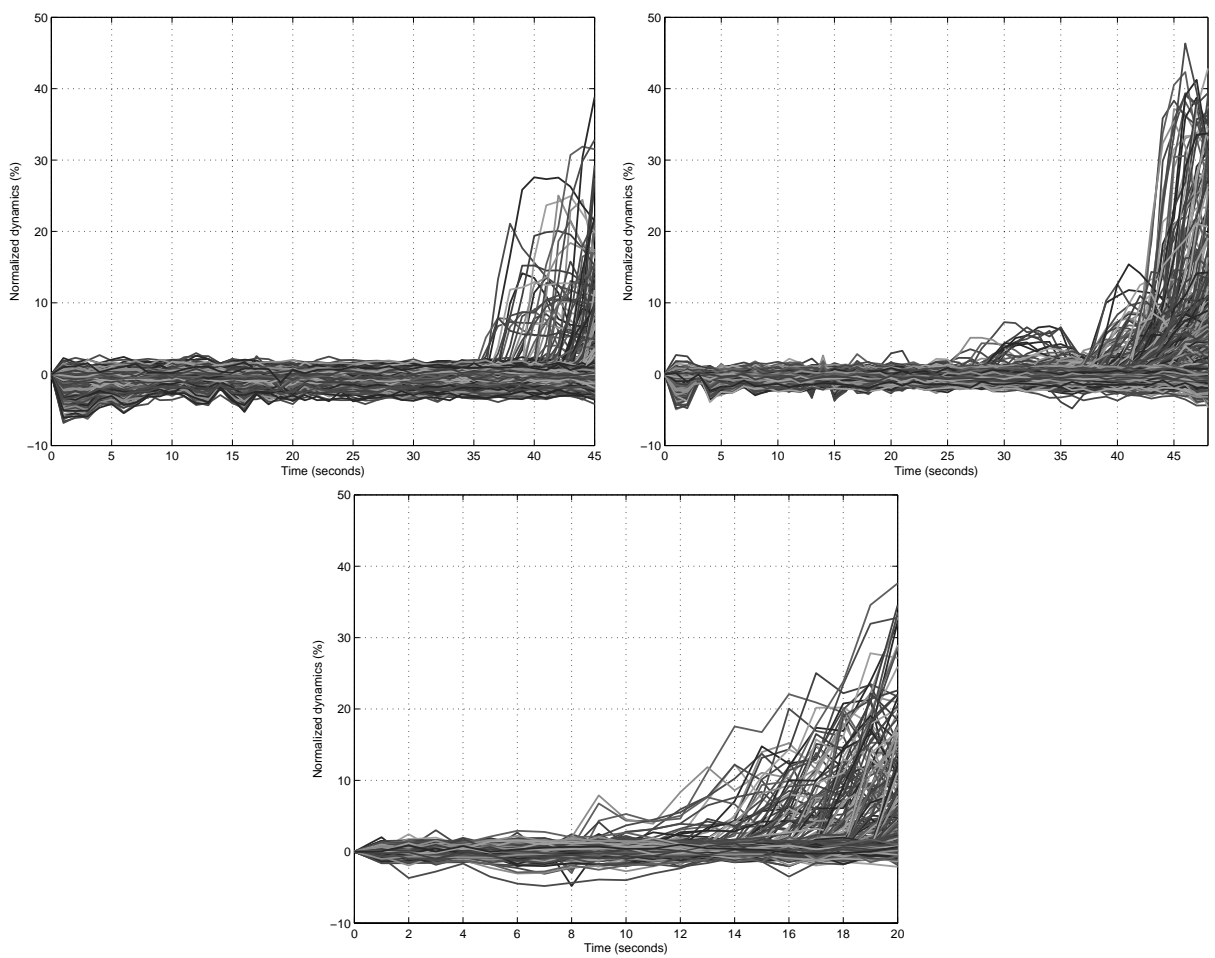

Figure 7.10: Normalized dynamics (\%) of all the regions composing the image plane division for three recordings until detecting smoke. 


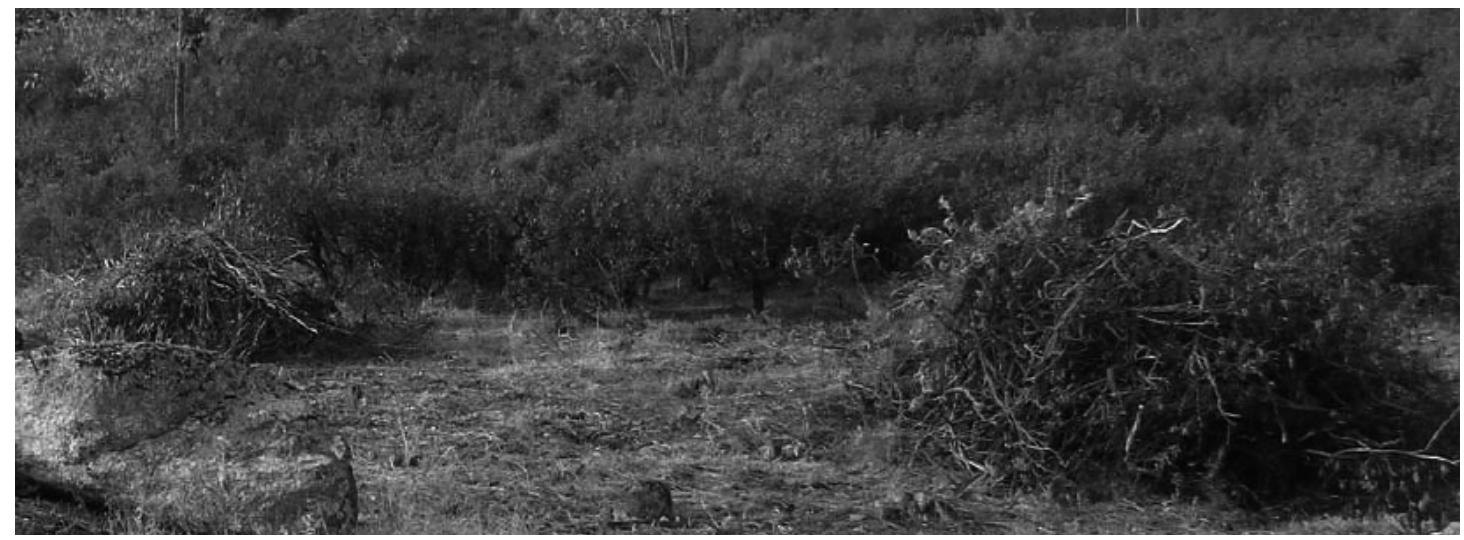

FiguRE 7.11: Forest debris burnt during the field tests.

the smart imager of the system. An accurate adjustment would demand elementary blocks of $3.66 \times 3 \mathrm{px}$. We finally decided to set blocks of $4 \times 4 \mathrm{px}$ for a slightly further image simplification. The algorithm was first tested in the laboratory. To this end, some of the video recordings containing smoke sequences were displayed on a computer screen towards which EyeRIS ${ }^{\mathrm{TM}}$ was focused. We also made sure that the evolution of the algorithm could be supervised in real time from a PC connected to EyeRIS ${ }^{\mathrm{TM}}$. This real-time supervision resulted very useful to detect minor problems during the field tests realized at the public forest "Las Navas-El Berrocal", located in the province of Seville $(37.85 \mathrm{~N}, 6.04 \mathrm{~W})$. Two controlled burns of forest debris like those of Fig. 7.11 were overseen by personnel of the Andalusian Forest Fire Supression and Prevention Service (INFOCA). The EyeRIS ${ }^{\mathrm{TM}}$ system was placed on top of a three-meter high pole powered by a commercial $9 \mathrm{~V}$ battery connected to a DC-to-DC converter in order to supply it the adequate voltage. A camcorder was also placed on top of the pole in order to record the sequences with the same perspective as EyeRIS ${ }^{\mathrm{TM}}$. The arrangement of both the EyeRIS ${ }^{\mathrm{TM}}$ system and the camcorder can be seen in Fig. 7.12. The pole was situated around 50 meters far from the forest debris. Besides this, another camcorder on a tripod was situated at different positions around the burns to record them from different perspectives. All the sequences can be found and downloaded from [V-MOTE Web Page].

Smoke was detected without false alarms in both burns. For the first one, the alarm was triggered at $2 \mathrm{~min} 50 \mathrm{sec}$ from ignition whereas for the second one the alarm was delivered at 57sec. Some consecutive frames captured by the EyeRIS ${ }^{\mathrm{TM}}$ during the first burn along with their corresponding candidate regions are represented in Fig. 7.13. The evolution of $N(t)$ and $Z(t)$ is depicted in Fig. 7.14 for both burns. The most remarkable aspect about the results is the ability of the algorithm to filter motion other than smoke. In fact, it can be seen from the image sequences extracted from EyeRIS ${ }^{\mathrm{TM}}$ [V-MOTE Web Page] that two potential sources of false alarms like the motion of tree leaves due to wind and the motion of people across the scene are mostly filtered. Thus, the alarms are undoubtedly triggered by the smoke arising from the burns. 

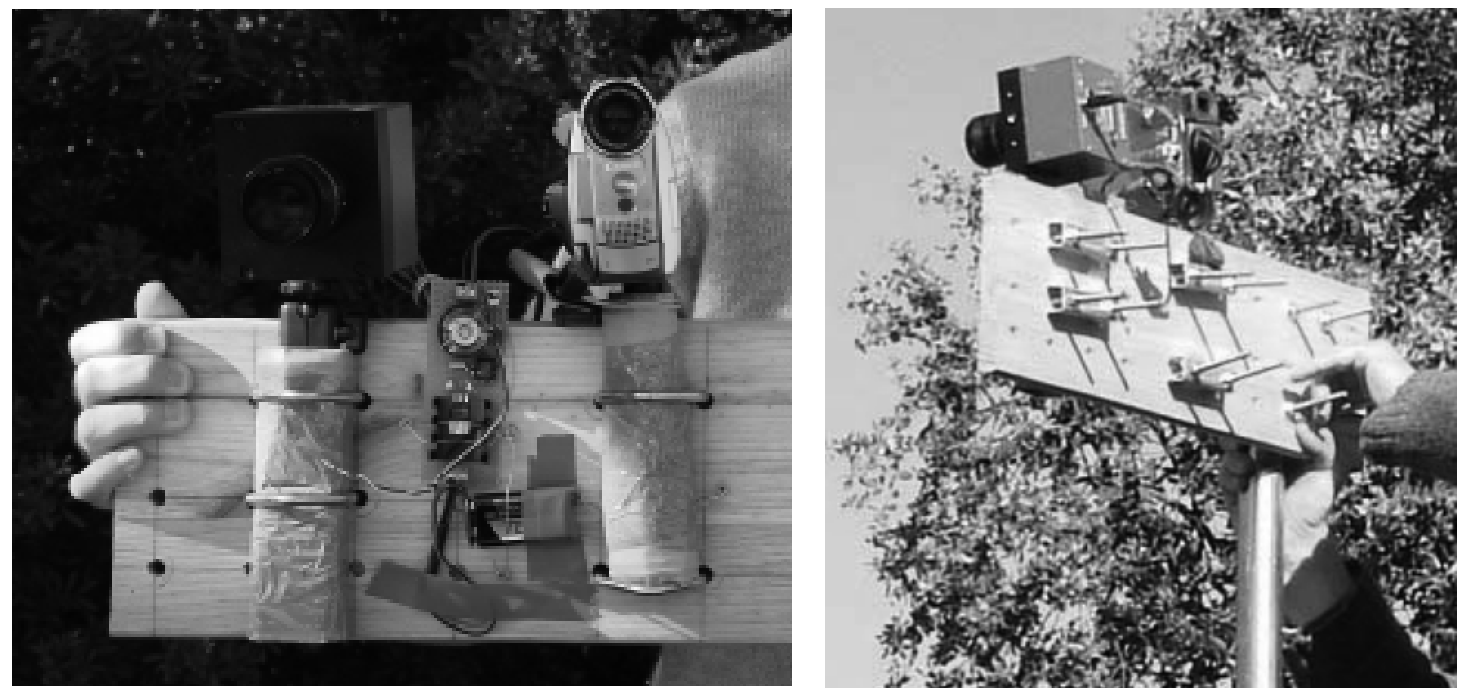

Figure 7.12: Arrangement of the EyeRIS ${ }^{\mathrm{TM}}$ system (left at both photos) and the commercial camcorder (right at both photos) during the field tests.
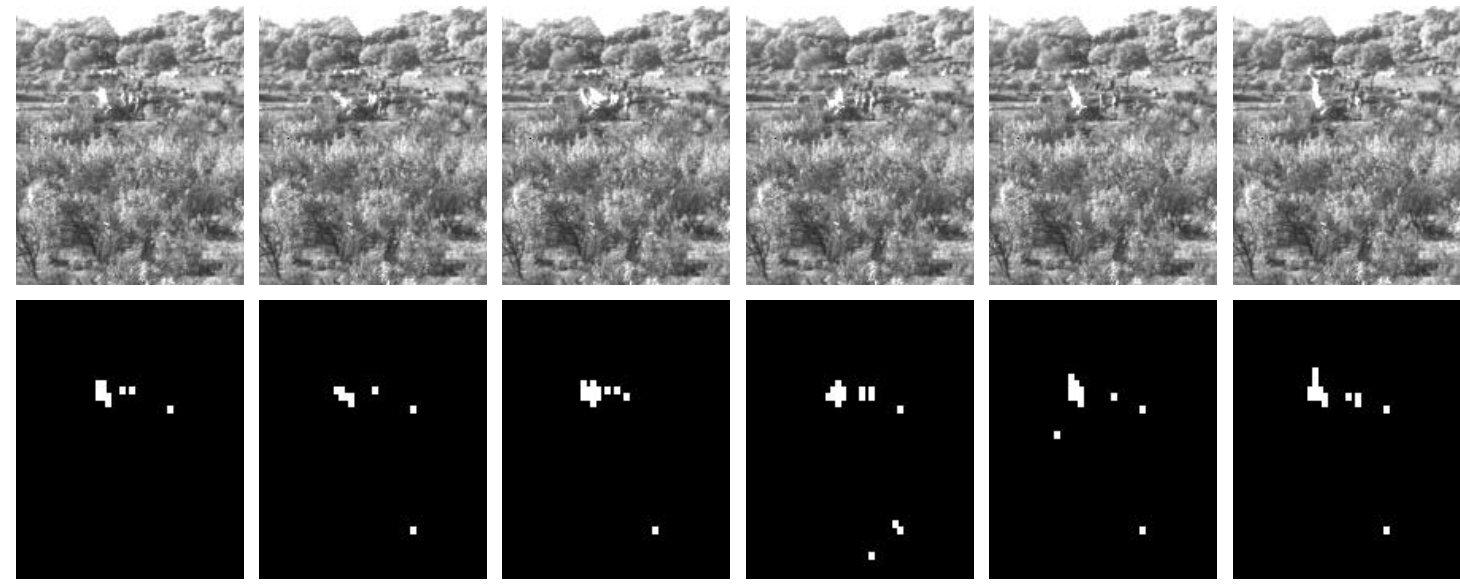

Figure 7.13: Consecutive frames captured by the EyeRIS ${ }^{\mathrm{TM}}$ (first row) and their corresponding candidate bins (second row) during the first controlled burn.
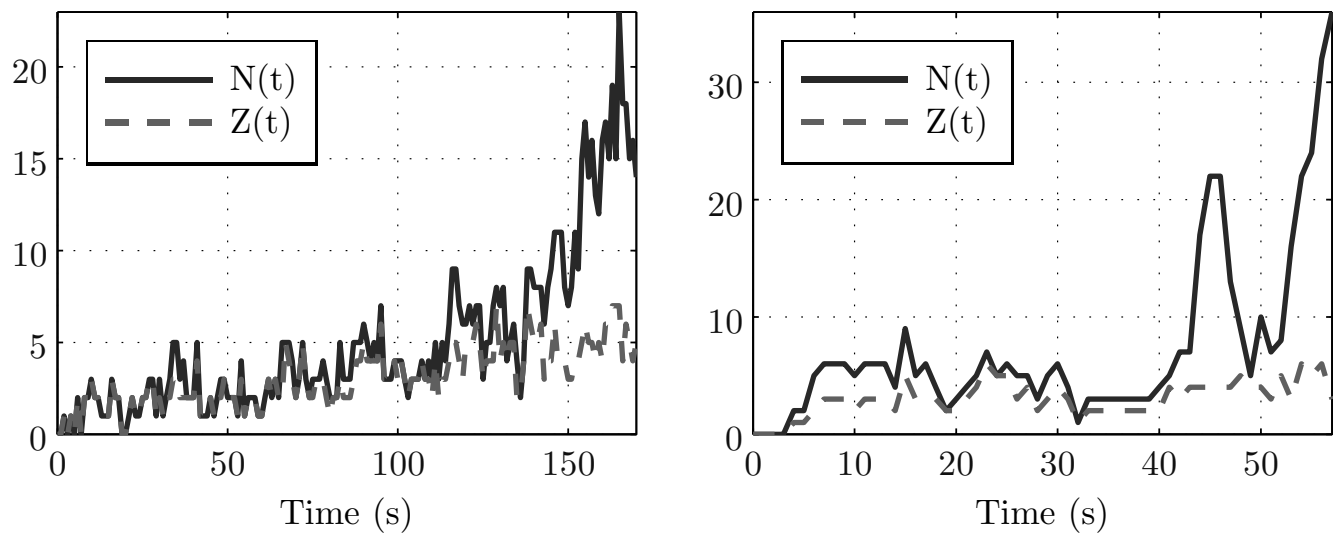

Figure 7.14: Evolution of $N(t)$ and $Z(t)$ for the first and second burn respectively. 


\subsection{Tests with $W i-F L I P^{1}$}

Once the suitability of the algorithm for on-site early detection of forest fires was confirmed, we programmed it into Wi-FLIP. In this case, no additional modification was necessary as the resolution of FLIP-Q is the same as Q-Eye. Furthermore, the adaptation of the code written for EyeRIS $S^{\mathrm{TM}}$ to $W i$-FLIP was quite simple as $\mathrm{C}$ dialects are used in both systems. In order to reach the prescribed frame rate of the algorithm, 1fps, working with quarter-resolution images, the PXA271 processor must be set to $416 \mathrm{MHz}$. It is translated into a power consumption of around $155 \mathrm{~mA}$. Thanks to the availability of radio communication in Wi-FLIP, full-resolution images are constantly sent via radio every 15 s since an alarm is triggered. New tests were first carried out at the public park Parque del Alamillo. Commercial pyrotechnics were again used as smoke generators. Strong gusts of wind made the arrangement of these tests complicated. In any case, no false alarm was triggered during eight sequences of smoke generation and the detection was successful for five of them. For the rest, smoke was not successfully detected as, due to the wind, it did not enter the field of view of FLIP-Q enough before the pyrotechnic material burnt out. For a real fire, smoke steadily spreads and therefore it should be eventually detected. In Fig. 7.15, some frames captured by a commercial camcorder and the corresponding smoke segmentation realized by $W i-F L I P$ are shown. The last image corresponds to the first alarm image sent via radio.
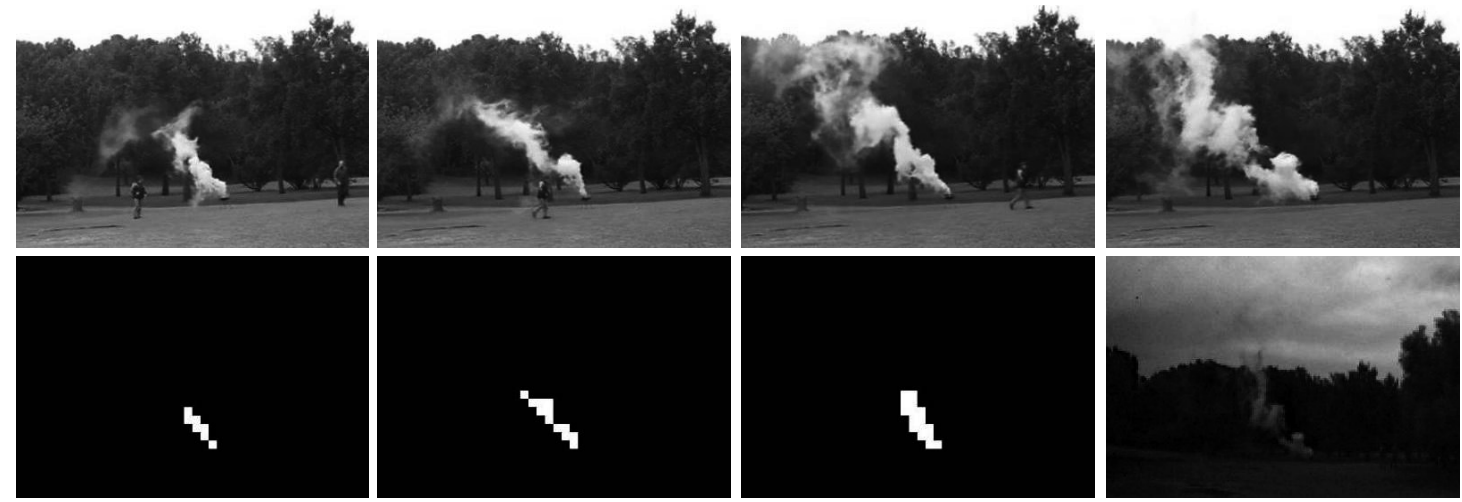

FigURE 7.15: Smoke segmentation and alarm image from Wi-FLIP.

The latest test consisted of the prescribed burn of a $95 \mathrm{~m} 20 \mathrm{~m}$ vegetation area at the public forest "Las Navas-El Berrocal", again in collaboration with the Andalusian Forest Fire Supression and Prevention Service. This area featured a smooth slope and different species of shrub: Genista hirsuta, Lavandula stoechas and Cistus ladanifer. They constitute fuel model 6 according to the Rothermel fire-spread model [Rothermel, 1972]. Wi-FLIP was placed about 80m away from the just described area and monitored all the activity occurring in it for over two hours. Also a

1 (C)2011 IEEE. Reprinted, with permission, from J. Fernández-Berni et al. "Wi-FLIP: A Wireless Smart Camera Based on a Focal-plane Low-power Image Processor", Fifth ACM/IEEE International Conference on Distributed Smart Cameras, pp. 1-4, Ghent, Belgium, August 22-25, 2011. 

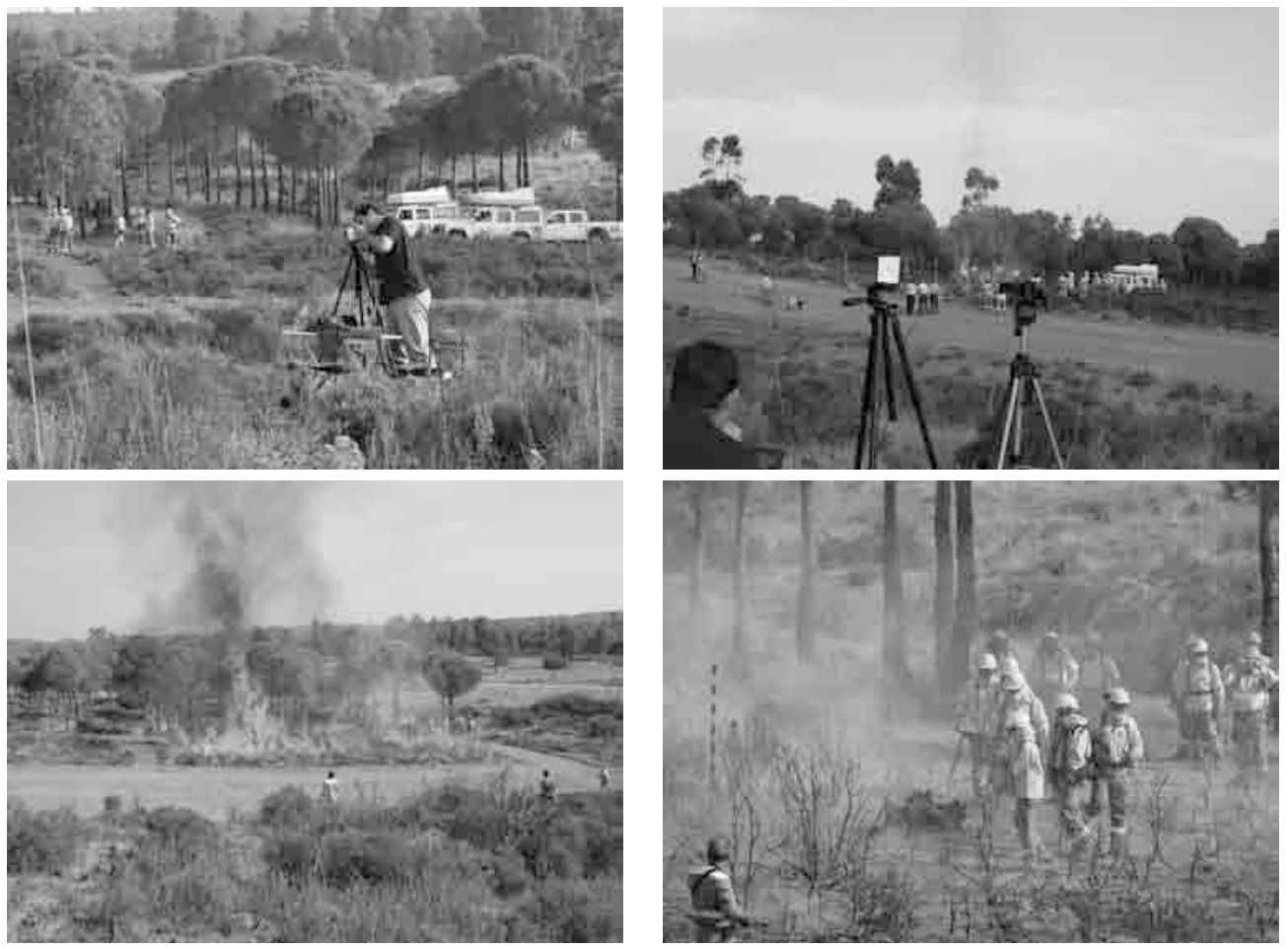

FiguRE 7.16: Snapshots taken during the latest test carried out.

commercial camcorder recorded a number of sequences. Some snapshots taken during the test are shown in Fig. 7.16. More material can be found [V-MOTE Web Page]. Concerning the results achieved, we must first mention that, before starting the burn, the area was mechanically divided into three zones of similar sizes according to the density of vegetation. The first zone presented really sparse vegetation. This meant that very thin smoke was generated, being the only case in which Wi-FLIP did not trigger an alarm. For the second zone, the vegetation was denser and the smoke arising was therefore thicker. Thus, a first alarm was triggered at $5 \mathrm{~m} 28 \mathrm{sec}$ since ignition. With the fire still active, we reset Wi-FLIP so that the algorithm started to run again from scratch. A new alarm was triggered $2 \mathrm{~m} 40 \mathrm{sec}$ after this reset operation. Finally, the third zone featured the densest vegetation. A first alarm was delivered 3m 29sec after the fire was initiated in it. Then, we repeated the reset operation of Wi-FLIP performed for the second zone, triggering a second alarm $1 \mathrm{~m} 9 \mathrm{sec}$ afterwards. These alarms even with the fire already spreading demonstrate the capability of the algorithm to detect the presence of fire in a scene, no matter its characteristics at the moment. The first images sent via radio by Wi-FLIP for two of the four alarms triggered are depicted in Fig. 7.17. It is also especially remarkable that, despite the fact that a great deal of people and vehicles were moving around, no false alarm was triggered neither before nor after the prescribed burn. This is a key point concerning the reliability of our vision-enabled WSN node. 

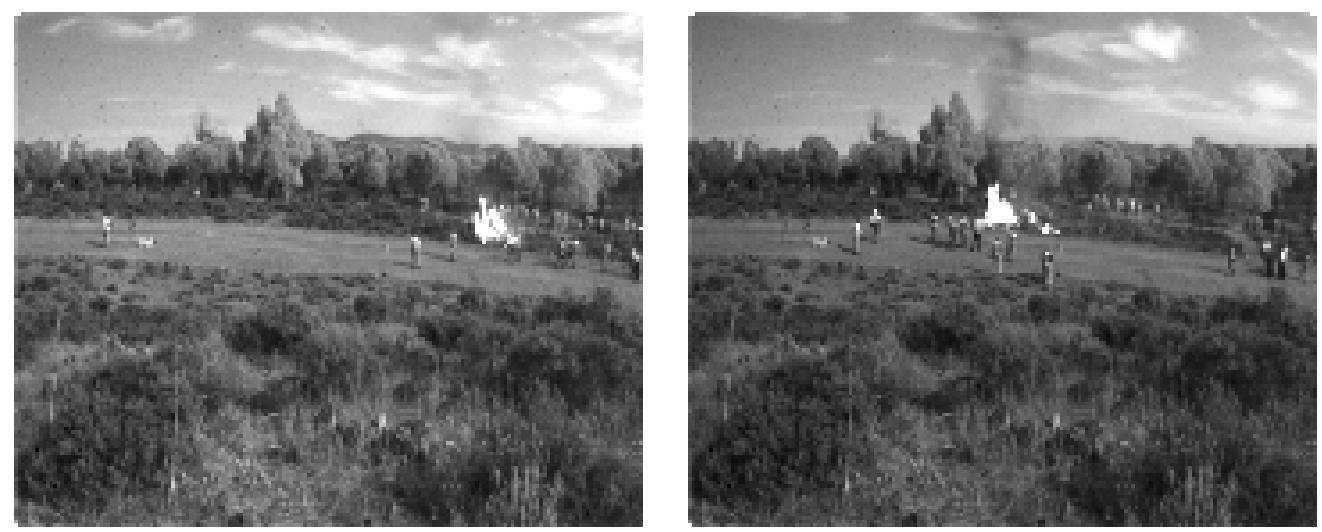

FiguRE 7.17: Images sent via radio by Wi-FLIP for two of the alarms triggered.

\subsection{Summary}

The incorporation of vision hardware into the nodes means a decisive step forward for WSNs. Apart from future applications, those ones currently being studied by the research community, e.g. environmental monitoring, take a new dimension in which appealing possibilities arise to be explored. In this final chapter, we address the application of the work described throughout this book to the field of forest fire detection. A new framework is defined to carry out this detection with greater robustness and reliability than in current camera-based systems. Also a vision algorithm tailored for this framework has been developed. The results achieved from numerous tests in different scenarios have demonstrated the suitability of the approach proposed. 



\section{Bibliography}

Abbo, A.A., Kleihorst, R.P., Choudhary, V., Sevat, L., Wielage, P., Mouy, S., Vermeulen, B., \& HeiJligers, M. 2008. Xetal-II: A 107 GOPS, 600 mW Massively Parallel Processor for Video Scene Analysis. IEEE J. Solid-State Circuits, 43(1), 192-201.

ADCM-1700 (Agilent). Agilent Camera Module. www.tvsat.com.pl/pdf/A/adcm-1700_agi.pdf.

ADCM-2700 (Agilent). Agilent Camera Module. http://www.alldatasheet.com/datasheet-pdf/pdf/137463/HP/ADCM-2650-0001.html.

ADns-3060 (Agilent). Agilent High Performance Optical Mouse Sensor. http://www.alldatasheet.com/datasheet-pdf/pdf/132520/HP/ADNS-3060.html.

Akyildiz, I.F., Weilian, S., Sankarasubramaniam, Y., \& Cayirci, E. 2002. A Survey on Sensor Networks. IEEE Communication Magazine, 40(8), 102-114.

Akyildiz, I.F., Melodia, T., \& Chowdhury, K.R. 2007. A Survey on Wireless Multimedia Sensor Networks. Computer Networks, 51(4), 921-960.

Andreou, A., \& Bohhen, K. 1995. A 590,000 Transistor 48,000 Pixel, Contrast Sensitive, Edge Enhancing, CMOS Imager-silicon Retina. Pages 225-240 of: Conference on Advanced Research in VLSI.

Arrue, B.C., Ollero, A., \& Martínez de Dios, J.R. 2000. An Intelligent System for False Alarm Reduction In Infrared Forest-Fire Detection. IEEE Intelligent Systems, 15(3), 64-73.

AT91SAM7S (ATMEL). ATMEL AT91SAM7S Microcontroller Family. http://www.atmel.com/dyn/resources/prod_documents/doc6175.pdf.

ATMega1281 (ATMEL). ATMEL Microcontroller Unit. http://www.atmel.com/dyn/products/product_card.asp?part_id=3630.

ATMEga128L (ATMEL). ATMEL Microcontroller Unit. http://www.atmel.com/atmel/acrobat/doc2467.pdf. 
ATMEL8051. ATMEL 8051 Architecture.

http://www.atmel.com/products/mcu8051/default.asp?category_id=163\&family_id=604\&source=left_nav.

AVHRR Web Page. Advanced Very High Resolution Radiometer (AVHRR).

http://noaasis.noaa.gov/NOAASIS/ml/avhrr.html.

Bakkali, M., Carmona-Galán, R., \& Rodríguez-Vázquez, A. 2010. A Prototype Node for Wireless Vision Sensor Network Applications Development. In: 5th International Symposium on I/V Communications and Mobile Network (ISVC).

Barrenetxea, G., Ingelrest, F., Schaefer, G., \& Vetterli, M. 2008. The Hitchhiker's Guide to Successful Wireless Sensor Network Deployments. Pages 43-56 of: Proc. of 6th Int. Conf. on Embedded Network Sensor Systems (SenSys).

Battistelli, E., Butera, F., Gonzalo, J., \& Jimenez-Tuñón, L. 2005. FuEGOSAT: the Space Payload for Fire Observation. Pages 134-139 of: 2nd International Conference on Recent Advances in Space Technologies, (RAST'05).

Brajovic, V., \& Kanade, T. 1998. Computational sensor for visual tracking with attention. IEEE J. Solid-State Circuits, 33(8), 1199-1207.

Cahoon, D.R., Stocks, B.J., Alexander, M.E., Baum, B.A., \& Goldammer, J.G. 2000. Wildland Fire Detection from Space: Theory and Application. Pages 151-169 of: Biomass Burning and its Inter-Relationships with the Climate System. Advances in Global Change Research. Kluwer Academic Publishers.

CC2420 (TEXAS Instr.). Texas Instruments IEEE802.15.4-compliant RF transceiver. http://focus.ti.com/docs/prod/folders/print/cc2420.html.

Chaczko, Z., \& Ahmad, F. 2005. Wireless Sensor Network Based System for Fire Endangered Areas. Pages 477-484 of: Third International Conference on Information Technology and Applications (ICITA'05).

Chen, P., Ahammad, P., Boyer, C., H., Shih-I, L., Leon, Lobaton, E., Meingast, M., O., Songhwai, Wang, S., Y., Posu, Yang, A.Y., Y., Chuohao, C., Lung-Chung, Tygar, J.D., \& SAstry, S.S. 2008. CITRIC: A Low-Bandwidth Wireless Camera Network Platform. In: ACM/IEEE Int. Conf. on Distributed Smart Cameras (ICDSC).

Chen, T., Yin, Y., Huang, S., \& Ye, Y. 2006. Smoke Detection for Early Fire-Alarming System Based on Video Processing. Pages 427-430 of: IEEE International Conference on Intelligent Information Hiding and Multimedia Signal Processing.

Chin-Chi, C., Chia-Hua, L., Chung-Te, L., \& Liang-Gee, C. 2009. iVisual: An Intelligent Visual Sensor SoC With 2790 FPS CMOS Image Sensor and 205 GOPS/W Vision Processor. IEEE J. Solid-State Circuits, 44(1), 127-135. 
Chua, L., \& Roska, T. 1993. The CNN Paradigm. IEEE Trans. Circuits Syst. I, 40(3), $147-156$.

ChuA, L., \& Roska, T. 2002. Cellular Neural Networks and Visual Computing: Foundation and Applications. New York, NY (USA): Cambridge University Press.

Cucchiara, R. 2005. Multimedia Surveillance Systems. Pages 3-10 of: Proc. of 3rd Int. Workshop on Video Surveillance $\mathcal{G}$ Sensor Networks (VSSN).

DE VRIES, J.S., \& Kemp, R.A. 1994. Results with a Multispectral Autonomous Wildfire Detection System. Proc. SPIE, Infrared Technology XX, 2269, 18-28.

den Breejen, E., Breuers, M., Cremer, F., Kemp, R.A., Roos, M., Schutte, K., \& DE VRIES, J.S. 1998. Autonomous Forest Fire Detection. Pages 2003-2012 of: 3rd International Conference on Forest Fire research, vol. 2.

Doolin, D.M., \& Sitar, N. 2005. Wireless Sensors for Wildfire Monitoring. Pages 477-484 of: Proc. of SPIE Symposium on Smart Structures and Materials.

Downes, I., Baghaei-Rad, L., \& Aghajan, H. 2006. Development of a Mote for Wireless Image Sensor Networks. Pages 360-369 of: Proc. of Cognitive Systems and Interactive Sensors (COGIS).

Dubois, J., Ginhac, D., Paindavoine, M., \& Heyrman, B. 2008. A 10000 FPS CMOS Sensor With Massively Parallel Image Processing. IEEE J. Solid-State Circuits, 43(3), 706717.

Dudek, P., \& Hicks, P.J. 2005. A general-purpose processor-per-pixel analog SIMD vision chip. IEEE Trans. Circuits Syst. I, 52(1), 13-20.

Eckert, J.P., \& Mauchly, J.W. 1947. ENIAC, U.S. Patent 3,120,606. First general-purpose electronic computer.

Eren, G., \& Akan, O.B. 2005. Multimedia Communication in Wireless Sensor Networks. Annales des Télécommunications, 60(7-8), 872-900.

Espejo, S., Carmona, R., Domínguez-Castro, R., \& Rodríguez-VÁzquez, Á. 1996. A VLSI-oriented Continuous-time CNN Model. International Journal of Circuit Theory and Applications, 24(3), 341-356.

Espina, J., Falck, T., \& Mulhens, O. 2006. Body Sensor Networks. Springer. Pages 169-174.

FAO. 2006. Global Forest Resources Assessment 2005. Tech. rept. Food and Agriculture Organization of the United Nations (FAO).

FAO. 2007. Fire management - global assessment 2006. Tech. rept. Food and Agriculture Organization of the United Nations (FAO). 
Fire Watch. 2004. Fire Watch: a commercial forest fire detection system. http://www.fire-watch.de.

Fleming, J., \& Robertson, R. 2003. Fire Management Tech Tips: The Osborne Fire Finder. Tech. rept. United States Department of Agriculture Forest Service.

Fulmar System Web Page. Fulmar Mini-UAS.

http://www.aerovision-uav.com.

Gay, David, levis, P., von Behren, R., Welsh, M., Brewer, E., \& Culler, D. 2003. The nesC Language: A Holistic Approach to Networked Embedded Systems. Pages 1-11 of: Proc. of Conf. on Programming Language Design and Implementation (PLDI).

Geelen, B., Deboeverie, F., \& Veelaert, P. 2009. Implementation of Canny Edge Detection on the WiCa SmartCam Architecture. In: ACM/IEEE Int. Conf. on Distributed Smart Cameras (ICDSC).

Gómez-Rodríguez, F., Pascual-Pea, S., Arrue, B.C., \& Ollero, A. 2002. Smoke Detection Using Image Processing. In: IV International Conference on Forest Fire Research.

González, R., \& Woods, R. 2002. Digital Image Processing. Prentice Hall.

Green, W. 1975. Are they real? Byte Magazine, 61,81,and 87.

Gross, N. 1999. 21 ideas for the 21st century: INTERNET. BusinessWeek, Aug. 30, 78-167.

Hefeeda, M. 2007. Forest Fire Modeling and Early Detection using Wireless Sensor Networks. Tech. rept. School of Computing Science, Simon Fraser University.

Hengstler, S., Prashanth, D., Sufen, F., \& Aghajan, H. 2007. Mesheye: a HybridResolution Smart Camera Mote for Applications in Distributed Intelligent Surveillance. Pages 360-369 of: Proc. of 6th Int. Conf. on Information Processing in Sensor Networks.

Hui, K., \& Shi, B. 1999. Distortion in Analog Networks for Image Filtering. IEEE Trans. Circuits Syst. I, 46(10), 1161-1171.

IBB2400 (MEMSIC). MEMSIC Wireless Sensor Network Components. http://www.memsic.com/support/documentation/wireless-sensor-networks/category/7datasheets.html?download=134\%3Aimote2.

IEEE STD 802.15.4. 2006. IEEE Std 802.15.4-2006: Wireless Medium Access Control (MAC) and Physical Layer (PHY) Specifications for Low-Rate Wireless Personal Area Networks (WPANs). Revision of IEEE Std 802.15.4-2003.

IIB2400 (MEMSIC). MEMSIC Wireless Sensor Network Components. http://www.memsic.com/support/documentation/wireless-sensor-networks/category/7datasheets.html?download=133\%3Aiib2400. 
Imote2 (MEMSIC). MEMSIC Wireless Sensor Network Modules.

http://www.memsic.com/products/wireless-sensor-networks/wireless-modules.html.

INSA. 2000. FUEGO Instrument Design, Prototype, Construction and Validation. Tech. rept. INSA Ingeniería y Servicios Aeroespaciales.

IRIS (MEMSIC). MEMSIC Wireless Sensor Network Modules.

http://www.memsic.com/products/wireless-sensor-networks/wireless-modules.html.

Jaehyuk, C., Sang-Wook, H., Seong-Jin, K., Sun-Il, C., \& Euisik, Y. 2007. A SpatialTemporal Multiresolution CMOS Image Sensor With Adaptive Frame Rates for Tracking the Moving Objects in Region-of-Interest and Suppressing Motion Blur. IEEE J. Solid-State Circuits, 42(12), 2978-2989.

Jahne, B. 1999. Handbook of Computer Vision and Applications (volume 2). Academic Press. Chap. 4, pages 67-90.

Jahne, B. 2001. Digital Image Processing. Springer. Chap. 11.

Kemeny, S.E., Panicacci, R., Pain, B., Matthies, L., \& Fossum, E.R. 1997. Multiresolution image sensor. IEEE Transactions on Circuits and Systems for Video Technology, 7(4), $575-583$.

Kleihorst, R., Schueler, B., Danilin, A., \& Heijligers, M. 2006. Smart Camera Mote with High Performance Vision System. In: ACM SenSys Workshop on Distributed Smart Cameras $(D S C)$.

Kleihorst, R., Abbo, A., Schueler, B., \& Danilin, A. 2007. Camera Mote with a HighPerformance Parallel Processor for Real-Time Frame-Based Video Processing. Pages 69-74 of: IEEE Conference on Advanced Video and Signal Based Surveillance (AVSS).

Kobayashi, H., White, J., \& Abidi, A. 1991. An Active Resistor Network for Gaussian Filtering of Images. IEEE J. Solid-State Circuits, 26(5), 738-748.

Komuro, T., Iwashita, A., \& Ishikawa, M. 2009. A QVGA-Size Pixel-Parallel Image Processor for 1000-FPS Vision. IEEE Micro, 29(6), 58-67.

Krstinic, D., Stipanicev, D., \& Jakovcevic, T. 2009. Histogram-based Smoke Segmentation in Forest Fire Detection System. Information Technology and Control, 38(3), 237-244.

Kuhrt, E., Knollenberg, J., \& Mertens, V. 2001. An Automatic Early Warning System for Forest Fires. Annals of Burns and Fire Disasters, XIV(3), 151-155.

Kulkarni, V., Forster, A., \& Venayagamoorthy, G. 2010. Computational Intelligence in Wireless Sensor Networks: A Survey. IEEE Communications Surveys $\&$ Tutorials. To be published, only electronic version currently available. 
Kwanho, K., Seunguin, L., Joo-Young, K., Minsu, K., \& Hoi-Jun, Y. 2009. A 125 GOPS 583 mW Network-on-Chip Based Parallel Processor With Bio-Inspired Visual Attention Engine. IEEE J. Solid-State Circuits, 44(1), 136-147.

Langendoen, K., Baggio, A., \& Visser, O. 2006. Murphy Loves Potatoes: Experiences from a Pilot Sensor Network Deployment in Precision Agriculture. In: 20th Int. Parallel and Distributed Processing Symposium (IPDPS).

Leñero, J., T., Serrano-Gotarredona, \& B., Linares-Barranco. 2009. A Mismatch Calibrated Bipolar Spatial Contrast AER Retina with Adjustable Contrast Threshold. Pages 1493-1496 of: International Symposium on Circuits and Systems (ISCAS).

Levis, P., \& GAY, D. 2009. TinyOS Programming. New York, NY (USA): Cambridge University Press.

Liñán, G., Rodríguez-VÁzquez, A., Carmona-Galán, R., Jímenez-Garrido, F., EsPejo, S., \& Domínguez-CAstro, R. 2004. A 1000 FPS at 128x128 vision processor with 8-bit digitized I/O. IEEE J. of Solid-State Circuits, 39(7), 1044-1055.

Lindeberg, T. 1991. Discrete Scale-Space Theory and the Scale-Space Primal Sketch. Ph.D. thesis, Royal Institute of Technology, Stockholm, Sweden.

Lindeberg, T. 1994. Scale-space Theory: A Basic Tool for Analysing Structures at Different Scales. Journal of Applied Statistics, 21(2), 225-270.

LPC2106 (PHILIPs). Philips LPC2104/2105/2106 Microcontroller Family. http://www.nxp.com/documents/data_sheet/LPC2104_2105_2106.pdf.

Machado, R., Ribeiro, G., Ditmore, A., Romano, M., \& Meireles, W. 2010. Online Paradise: A Wireless Sensor Network Applied in the Prevention and Detection of Forest Fires. In: VI International Conference on Forest Fire Research.

Malhi, Y., Meir, P., \& Brown, S. 2002. Forests, Carbon and Global Climate. Philosophical Transactions: Mathematical, Physical and Engineering Sciences, 360(1797), 1567-1591.

Masland, R.H. 2001. The Fundamental Plan of the Retina. Nature Neuroscience, 4(9), 877886.

McCulloch, J., McCarthy, P., Guru, S.M., Peng, W., Hugo, D., \& Terhorst, A. 2008. Wireless Sensor Network Deployment for Water Use Efficiency in Irrigation. Pages 46-50 of: Proc. of Workshop on Real-World Wireless Sensor Networks (REALWSN).

MeAD, C. 1989. Analog VLSI and Neural Systems. Addison-Wesley. 
Merino, L., Caballero, F., de Dios J.R., Martínez, Maza, I., \& Ollero, A. 2010. Automatic Forest Fire Monitoring and Measurement using Unmanned Aerial Vehicles. In: VI International Conference on Forest Fire Research. ref. 292.

MIB520 (MEMSIC). MEMSIC Wireless Sensor Network Gateways. http://www.memsic.com/products/wireless-sensor-networks/gateways.html.

MICA2 (MEMSIC). MEMSIC Wireless Sensor Network Modules. http://www.memsic.com/products/wireless-sensor-networks/wireless-modules.html.

MODIS Web PAge. Moderate Resolution Imaging Spectroradiometer (MODIS). http://modis.gsfc.nasa.gov/.

Moore, G.E. 1965. Cramming More Components onto Integrated Circuits. Electronics, 38(8), $114-117$.

Mutch, J., \& Lowe, D. 2008. Object Class Recognition and Localization Using Sparse Features with Limited Receptive Fields. Int. J. of Computer Vision, 80(1), 45-57.

NI, Y. 2005. Smart image sensing in CMOS technology. IEE Proc.-Circuits Devices Syst., 152(5), $547-555$

Ni, Y., \& GuAN, J. 2000. A 256x256 pixel smart CMOS image sensor for line-based stereo vision applications. IEEE J. Solid-State Circuits, 35(7), 1055-1061.

Ni, Y., Zhu, Y., Arian, B., \& Devos, F. 1993. Yet Another Analog 2D Gaussian Convolver. Pages 192-195 of: International Symposium on Circuits and Systems (ISCAS).

Nilchi, A., AzIz, J., \& Genov, R. 2009. Focal-Plane Algorithmically-Multiplying CMOS Computational Image Sensor. IEEE J. Solid-State Circuits, 44(6), 1829 -1839.

NIOS-II (Altera). Altera NIOS-II processor. http://www.altera.com/products/ip/processors/nios2/ni2-index.html

OM6802 (Philips). Philips Camera Module. http://thekef.free.fr/CV/OM6802.pdf.

on Biological Diversity, Convention. 2001. Global Diversity Outlook. Tech. rept. United Nation Environment Programme (UNEP).

OsBorne, A. 1981. Osborne I. First commercially successful portable microcomputer.

OV6620 (Omnivision). Omnivision Camera Module. http://www.datasheetarchive.com/ov6620-datasheet.html.

OV7649 (Omnivision). Omnivision Camera Module. http://www.datasheetarchive.com/ov7649-datasheet.html. 
OV9655 (OMnivision). Omnivision Camera Module.

http://www.datasheetarchive.com/ov9655-datasheet.html.

Papoulis, A., \& Unnikrishna, S. 2002. Probability, Random Variables and Stochastic Processes. McGraw Hill.

Parque del Alamillo Web Page. Parque del Alamillo de Sevilla. http://www.parquedelalamillo.org/.

Poggio, T., Voorhees, H., \& Yuille, A. 1988. A Regularized Solution to Edge Detection. J. of Complexity, 4(2), 106-123.

Poikonen, J., Laiho, M., \& PaAsio, A. 2009. MIPA4k: A 64x64 Cell Mixed-mode Image Processor Array. Pages 1927-1930 of: IEEE International Symposium on Circuits and Systems $(I S C A S)$.

Pottie, G.J., \& Kaiser, W.J. 2000. Wireless Integrated Network Sensors. Communications of the $A C M, \mathbf{4 3}(5), 51-58$.

Poynton, C. 2007. Digital Video and HDTV: Algorithms and Interfaces. Elsevier Science.

PXA270 (MARVell). Marvell Applications Processors.

http://www.marvell.com/products/processors/applications/pxa_family/.

PXA271 (MARVEll). Marvell Applications Processors.

http://www.marvell.com/products/processors/applications/pxa_family/.

Rabaey, J. 1995. Digital Integrated Circuits: A Design Perspective. Prentice Hall.

Raffo, L., Sabatini, S., Bo, G., \& Bisio, G. 1998. Analog VlSI Circuits as Physical Structures for Perception in Early Visual Tasks. IEEE Trans. on Neural Networks, 9(6), $1483-1494$

Rahimi, M., Baer, R., Iroezi, O.I., Garcia, J.C., Warrior, J., Estrin, D., \& Srivastava, M. 2005. Cyclops: In Situ Image Sensing and Interpretation in Wireless Sensor Networks. Pages 192-204 of: Proc. of 3rd Int. Conf. on Embedded Networked Sensor Systems (SenSys).

Rodríguez-Vázquez, A., Domínguez-Castro, R., Jímenez-Garrido, F., Morillas, S., Listán, J., Alba, L., Utrera, C., Espejo, S., \& Romay, R. 2008. The Eye-RIS CMOS vision system. Chap. 2 of: CAsier, H., Steyaert, M., \& Roermund, A. (eds), Sensors, Actuators and Power Drivers; Integrated Power Amplifiers from Wireline to RF; Very High Frequency Front Ends. Analog Circuit Design. Springer.

Roska, B., \& Werblin, F. 2001. Vertical Interactions across Ten Parallel, Stacked Representations in the Mammalian Retina. Nature, 410, 583-587. 
Rothermel, R.C. 1972. Forest Fire Modeling and Early Detection using Wireless Sensor Networks. Tech. rept. Intermountain Forest and Range Experiment Station, USDA Forest Fire Service.

Rowe, A., Mangharam, R., \& Rajkumar, R. 2006. FireFly: A Time Synchronized RealTime Sensor Networking Platform. Chap. 3 of: Tseng, Y., \& Wu, S. (eds), Wireless Ad Hoc Networking: Personal-Area, Local-Area, and the Sensory-Area Networks. CRC Press.

Rowe, A., Goode, A., Goel, D., \& Nourbakhsh, I. 2007a. CMUcam3: An Open Programmable Embedded Vision Sensor. Tech. rept. Carnegie Mellon Robotics Institute.

Rowe, A., Goel, D., \& Rajkumar, R. 2007b. FireFly Mosaic: A Vision-Enabled Wireless Sensor Networking System. Pages 459-468 of: 28th IEEE International Real-Time Systems Symposium, RTSS.

San-Miguel-Ayanz, J., Ravail, N., Kelha, V., \& Ollero, A. 2005. Active Fire Detection for Fire Emergency Management: Potential and Limitations for the Operational Use of Remote Sensing. Natural Hazards, 35(3), 361-376.

Schroeder, D. 2004. Evaluation of Three Wildfire Smoke Detection Systems. Advantage Report, FERIC Forest Engineering Research Institute of Canada, 5(24).

Schroeder, D. 2005. Operational Trial of the ForestWatch Wildfire Smoke Detection System. Advantage Report, FERIC Forest Engineering Research Institute of Canada, 6(17).

SHI, B. 2009. The Effect of Mismatch in Current- versus Voltage-mode Resistive Grids. Int. J. of Circuit Theory and Applications, 37(1), 53-65.

Shi, B., \& ChuA, L. 1992. Resistive Grid Image Filtering: Input/Output Analysis via the CNN Framework. IEEE Trans. Circuits Syst. I, 39(7), 531-548.

Siden, J., Koptyug, A., Gulliksson, M., \& Nilsson, H. 2007. An Action Activated and Self Powered Wireless Forest Fire Detector. Pages 47-58 of: Wireless Sensor and Actor Networks. IFIP International Federation for Information Processing. Springer Boston.

Sistema Bosque. 1999. Sistema Bosque (Andalusian Forest Fire Supression and Prevention Service). http://waste.ideal.es/sistemabosque.htm.

Son, B., Her, Y., \& Kim, J. 2006. A Design and Implementation of Forest-Fires Surveillance System Based on Wireless Sensor Networks for South Korea Mountains. Int. J. of Computer Science and Network Security, 6(9), 124-130.

Stipanicev, D., Stula, M., Krstinic, D., Seric, L., Jakovcevic, T., \& Bugaric, M. 2010. Advanced Automatic Wildfire Surveillance and Monitoring Network. In: VI International Conference on Forest Fire Research. ref. 53. 
Takahashi, N., Fujita, K., \& Shibata, T. 2009. A Pixel-Parallel Self-Similitude Processing for Multiple-Resolution Edge-Filtering Analog Image Sensors. IEEE Trans. Circuits Syst. I, 562(11), 2384-2392.

Teixeira, T., \& Savvides, A. 2008. Lightweight People Counting and Localizing for Easily Deployable Indoors WSNs. IEEE Journal of Selected Topics in Signal Processing, 2(4), 493502 .

Teixeira, T., Lymberopoulos, D., Culurciello, E., Aloimonos, Y., \& Savvides, A. 2006. A Lightweight Camera Sensor Network Operating on Symbolic Information. In: First Workshop on Distributed Smart Cameras.

TELOSB (MEMSIC). MEMSIC Wireless Sensor Network Modules. http://www.memsic.com/products/wireless-sensor-networks/wireless-modules.html.

Tolle, G., Polastre, J., Szewczyk, R., Culler, D., Turner, N., Tu, K., Burgess, S., Dawson, T., Buonadonna, P., Gay, D., \& Hong, W. 2005. A Macroscope in the Redwoods. Pages 51-63 of: Proc. of 3rd Int. Conf. on Embedded Networked Sensor Systems (SenSys).

Toreyin, U., Dedeoglu, Y., \& Enis-Cetin, A. 2007. Computer Vision Based Forest Fire Detection and Monitoring System. In: International Wildland Fire Conference.

Unger, S.H. 1958. A Computer Oriented toward Spatial Problems. Proceedings of the IRE, 46(10), 1744-1750.

Utkin, A.B., Lavrov, A.V., Costa, L., Simoes, F., \& Vilar, R.M. 2002. Detection of Small Forest Fires by LIDAR. Applied Physics B: Lasers and Optics, 74(1), 77-83.

Utkin, A.B., Lavrov, A.V., \& Vilar, R.M. 2010. LIDARs for forest fire detection. In: VI International Conference on Forest Fire Research. ref. 15.

V-MOTE Web PAgE. V-MOTE project.

http://www.imse-cnm.csic.es/vmote.

Vicente, J., \& Guillemant, P. 2002. An Image Processing Technique for Automatically Detecting Forest Fire. International Journal of Thermal Sciences, 41(12), 1113-1120.

Vittoz, E., \& Arreguit, X. 1993. Linear Networks Based on Transistors. Electronic Letters, $\mathbf{2 9}(3), 297-299$.

WAdE, R. 2003. 10 Emerging Technologies That Will Change the World: Wireless Sensor Networks. Technology Review, 106(1), 33-49.

Weiser, M. 1991. The Computer for the 21th Century. Scientific American, 265(3), 94-104. 
Werner-Allen, G., Lorincz, K., Ruiz, M., Marcillo, O., Johnson, J., Lees, J., \& Welsh, M. 2006. Deploying a Wireless Sensor Network on an Active Volcano. IEEE Internet Computing, 10(2), 18-25.

Yu, L., Wang, N., \& Meng, X. 2005. Real-time Forest Fire Detection with Wireless Sensor Networks. Pages 1214-1217 of: International Conference On Wireless Communications, Networking and Mobile Computing (WiMob'05).

Zhiqiang, L., Hoffman, M.W., Schemm, N., Leon-Salas, W.D., \& Balkir, S. 2008. A CMOS Image Sensor for Multi-Level Focal Plane Image Decomposition. IEEE Trans. Circuits Syst. I, 55(9), 2561-2572.

Zivkovic, Z., Kliger, V., Kleihorst, R., Danilin, A., Schueler, B., Arturi, G., Chang, C.C., \& Aghajan, H. 2008. Toward Low Latency Gesture Control Using Smart Camera Network. In: IEEE Workshop on Computer Vision and Pattern Recognition (CVPRW). 